Supporting information for:

\title{
Enantioselective $\mathrm{Si}-\mathrm{H}$ insertion Reactions of Diarylcarbenes for the Synthesis of Silicon-Stereogenic Silanes
}

\author{
Jake R. Jagannathan, James C. Fettinger, Jared T. Shaw*, and Annaliese K. Franz* \\ Department of Chemistry, One Shields Ave, University of California, Davis, CA 95616 \\ *jtshaw@ucdavis.edu*akfranz@ucdavis.edu*
}

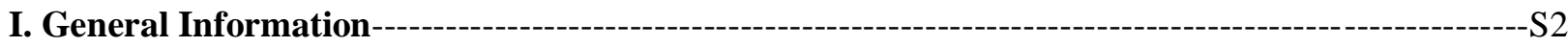

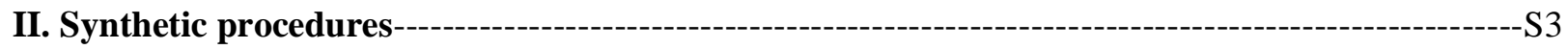

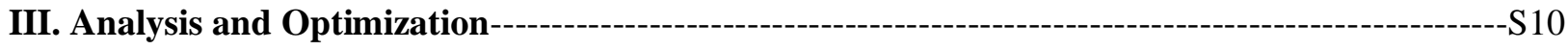

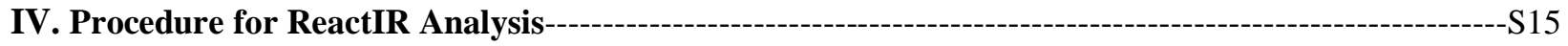

V. Procedure for kinetic isotope effect---

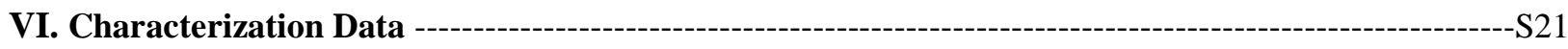

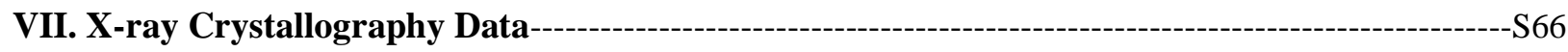

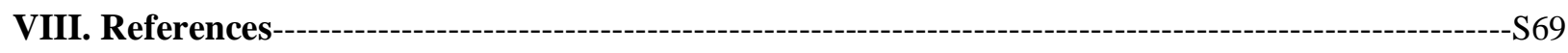

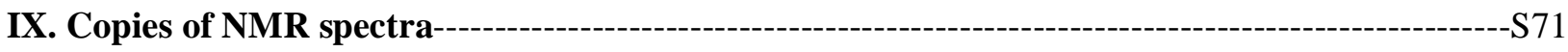


*CAUTION* Diazo compounds are high energy compounds and require careful treatment. We observed no problems throughout our work, but care should always be taken when handling large quantities of diazo compounds. See Bull et. al.1 for risk analysis for related diazo compounds.

\section{General Information}

The following abbreviations are used throughout: trimethylsilyl (TMS), Phenyltrimethylsilane (PhenylTMS), dirhodiumtetraacetate $\left(\mathrm{Rh}_{2}(\mathrm{OAc}) 4\right), \quad$ Tetrakis $[(R)$-(1-adamantyl)-(N-phthalimido)acetate $]$ dirhodium(II) $\left(\operatorname{Rh}_{2}(R\right.$ PTAD)4), $\quad$ Tetrakis $\left[(S)-(\mathrm{N}\right.$-phthalimido)-tert-lucinato $]$ dirhodium(II) $\quad\left(\mathrm{Rh}_{2}(S\right.$-PTTL $\left.) 4\right), \quad$ Tetrakis $[(S)$-(Nbiphenylphthalimido)-tert-lucinato]dirhodium(II) $\quad$ (Rh2(S-BPTTL)4), $\quad$ Tetrakis[(S)-N-(pdodecylphenylsulfonyl)prolinato]dirhodium(II) $\quad\left(\operatorname{Rh}_{2}(S\right.$-DOSP $\left.) 4\right), \quad$ Tetrakis[( $\left.S\right)-[(1 S)$-1-(4-bromophenyl)-2,2diphenylcyclopropanecarboxylato]dirhodium(II) $\quad\left(\mathrm{Rh}_{2}(S-\mathrm{BTPCP}) 4\right)$, Tetrakis[(S)-(N-tetrachlorophthalimido)-tertlucinato]dirhodium(II) ( $\mathrm{Rh}_{2}\left(S\right.$-TCPTTL)4), Dirhodium(II)tetrakis[methyl 2-pyrrolidone-5(S)-carboxylate], $\left(\mathrm{Rh}_{2}(5 R\right.$ MEPY)4), Tetrakis[N-phthalimido-(S)-valinato]dirhodium ( $\mathrm{Rh}_{2}(S$-PTV)4), ethyl acetate (EtOAc), isopropanol (IPA), tetrahydrofuran (THF), toluene (PhMe), dichloromethane (DCM), 1,2-dibromoethane (DBE), enantiomeric ratio (er), diastereomeric ratio $(\mathrm{dr})$.

\section{Materials.}

Cyclohexylphenylsilane,2 triethylsiloxyphenylsilane,3 2-methoxybenzophenone,4 naphthylphenylketone,5 2triflouoromethyl benzophenone,6 diphenyldiazomethane,6 4,4'-(diazomethylene)bis(chlorobenzene),7 4,4'(diazomethylene) bis(methoxybenzene) 7 were made from previously published procedures. ( $\mathrm{Rh}_{2}$ (S-TCPTTL) 4 was purchased from TCI; $\mathrm{Rh}_{2}(\mathrm{OAc})_{4}, \mathrm{Rh}_{2}(\mathrm{R}-\mathrm{PTAD})_{4}$ and $\mathrm{Rh}_{2}(\mathrm{~S}-\mathrm{BTPCP})_{4}$ purchased from Strem Chemicals $\mathrm{Inc}_{2} \mathrm{Rh}_{2}(S-$ $\mathrm{PTV})_{4}, \mathrm{Rh}_{2}\left(S\right.$-PTTL) $4,\left(\mathrm{Rh}_{2}(S \text {-BPTTL })_{4}\right.$ were donated by the Fox group. Dry $\mathrm{CH}_{2} \mathrm{Cl}_{2}, \mathrm{THF}, \mathrm{Et}_{2} \mathrm{O}$ and PhMe were dispensed from a solvent purification system that passes solvent through two columns of dry neutral alumina prior to use. All other reagents, unless otherwise noted, were purchased from commercial sources. NOTE: it is necessary that the $\mathrm{MnO}_{2}$ used for the oxidation of hydrazones be $\sim 85 \%$ pure with an average particle size of 2 microns, appearing as a fine black powder (e.g. Oakwood Chemical, CAS \#: 1313-13-9, cat. \#: 094454, lot \#: 094454K03K or Sigma Aldrich, cat: 217646-100G, Lot \# MKCJ7777).

\section{Synthesis, Purification and Analysis.}

All reactions were performed in oven-dried and argon-purged glassware (including $8 \mathrm{~mL}$ KIMAX Borosilicate glass vials fitted with rubber septa). Molecular sieves (4@) were activated by flame-drying, then cooled under vacuum ( $<1$ torr). All microwave experiments were run in a Biotage Initiator EXP EU 400W microwave synthesizer 2.0 (serial number 11031). Nuclear magnetic resonance (NMR) spectra were recorded at ambient temperature at 600, $400 \mathrm{MHz}$. Spectra obtained on Bruker Avance IIIHD Nanobay Spectrometer $(400 \mathrm{MHz}$ for $1 \mathrm{H} ; 100 \mathrm{MHz}$ for $13 \mathrm{C}$; $292 \mathrm{MHz}$ for ${ }_{19} \mathrm{~F} ; 79 \mathrm{MHz}$ for ${ }_{29} \mathrm{Si}$ ), and/or Varian VNMRS (600 MHz for ${ }_{1 \mathrm{H}} \mathrm{H} 151 \mathrm{MHz}$ for ${ }_{13} \mathrm{C}, 119 \mathrm{MHz}$ for ${ }_{29} \mathrm{Si}$ ). The ${ }_{1} \mathrm{H}$ spectral data are reported as follows: chemicals shifts were reported in parts per million downfield from tetramethylsilane internal standard on the $\delta$ scale, multiplicity (s, singlet; d, doublet; t, triplet; q, quartet; s, septet; m, multiplet; dd, doublet of doublets, and $\mathrm{b}$, broadened), coupling constant (Hz), and integration. Carbon NMR chemical shifts are reported in $\mathrm{ppm}$ from tetramethylsilane with the solvent reference employed as the internal standard (deuterochloroform $\left(\mathrm{CDCl}_{3}\right)$ at $77.16 \mathrm{ppm}$ ). All silicon NMR's were taken with $0.05 \mathrm{M} \mathrm{Cr}(\mathrm{Acac})_{3}$ as a $\mathrm{T}_{1}$ Relaxation agent.

Compound 9 was analyzed by high resolution mass spectrometry (HRMS) using Thermo Fisher Orbitrap (San Jose, CA) using electrospray in the negative or positive ion mode $>60,000$ resolution and using typical ESI source values. Mass spectrometry data was obtained at the Mass Spectrometry Facilities of University of California, Davis. Insertion products were also analyzed by mass spectrometry on a Bruker UltraFlextreme MALDI mass spectrometer (Bruker Corp, Billeraca, MA) in reflectron mode. Samples were first mixed in a 1:1 ratio with a saturated solution of alpha-hydroxycinnamic acid (Sigma Chemical Co) in high-purity water:MeCN (35\%:65\%) and $1 \mathrm{mg}$ of NaI to promote cationization was added before being spotted on the plate and allowed to air dry. Samples were analyzed using the minimum laser fluence to obtain adequate signal $(\mathrm{s} / \mathrm{n}>20)$, generally requiring 1000 shots per sample. Data was 
analyzed in FlexAnalysis. Diazo compounds 3a-3d were analyzed using low-resolution mass spectrometry with an Advion $\odot$ ASAP-APCI-MS was achieved and the corresponding data is reported for those samples. Several samples (including silanes) did not ionize with any technique used and are noted in their characterization data.

Kinetic Analysis was performed using a Mettler Toldedo ReactIR 700 (serial number B929971514) with a liquid $\mathrm{N}_{2}$ MCT detector fitted with a DiComp probe (serial number B939349478). The system was filled with liquid $\mathrm{N}_{2}$ and allowed to cool for $1 \mathrm{~h}$ before kinetic experiments begun. Initial trends were found using iC IR 7.1 and further analyzed using Microsoft Excel.

High performance liquid chromatography (HPLC) data were obtained on Shimadzu LC-20AB system with CHIRALPAK ${ }^{\circledR}$ AD-H column $(4.6 \times 250 \mathrm{~mm}, 5 \mu \mathrm{m})$, CHIRALPAK ${ }^{\circledR}$ OD-H column $(4.6 \times 250 \mathrm{~mm}, 5 \mu \mathrm{m})$ or CHIRALPAK ${ }^{\circledR}$ AS column $(4.6 \times 250 \mathrm{~mm}, 5 \mu \mathrm{m})$ and Shimadzu SPD-M20A photodiode array detector. Each HPLC sample was eluted at a constant flow rate with isocratic (90:10 hexanes/isopropanol)/hexanes or (90:10 heptane/isopropanol)/heptane system and $40{ }^{\circ} \mathrm{C}$ column oven temperature.

\section{General Synthetic Procedures}

\section{IIa. Procedures for Starting Material Syntheses}

\section{Method A: Synthesis of donor/donor diazo compounds}

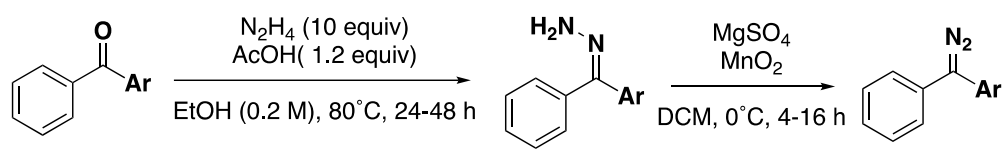

A 50-mL two-neck round-bottom flask with a stir bar and condenser was charged with ketone in anhydrous EtOH (0.2 M). Hydrazine (10 equiv), followed by $\mathrm{AcOH}$ (1.2 equiv) were added, the solution was heated to reflux. The reaction was monitored by TLC (7:3 hexanes/EtOAc) until the ketone was fully consumed. The reaction was allowed to cool to room temperature, and the solvent was removed via rotary evaporator. The resulting slurry was dissolved in $\mathrm{Et}_{2} \mathrm{O}(20 \mathrm{~mL})$, washed with $\mathrm{H}_{2} \mathrm{O}(10 \mathrm{~mL})$, brine $(10 \mathrm{~mL})$, and dried with $\mathrm{Na}_{2} \mathrm{SO}_{4}$. The solution was filtered, and the solvent was removed via rotary evaporator. The resulting hydrazone was verified to be present by TLC (7:3 hexanes EtOAc) and $1 \mathrm{H}$ NMR, then carried on without further purification.

The crude hydrazone mixture was transferred into a flame dried round bottom flask purged with argon and charged with a stir bar. DCM $(0.5 \mathrm{M})$ was added, followed by $\mathrm{MgSO}_{4}(100 \mathrm{mg} / \mathrm{mmol})$. The solution was cooled down to $0^{\circ} \mathrm{C}$, and $\mathrm{MnO}_{2}$ (8.0 equiv) was added in one portion. The solution was monitored by TLC ( $7: 3$ hexanes/ EtOAc) until all hydrazone was consumed. The solution was then filtered through celite, washed with DCM $(3 \times 10 \mathrm{~mL})$, and the solvent was removed via rotary evaporator. Diazo products were purified by column chromatography using (99:1 hexanes: $\mathrm{Et}_{3} \mathrm{~N}$ ) on basic alumina.

Diazo compounds 3a-d were found to be stable for $>48 \mathrm{~h}$ at room temperature when stored under argon, and $\sim 6$ weeks at $-23^{\circ} \mathrm{C}$. Diazo compound $2 \mathrm{a}$ was found to readily decompose within $24 \mathrm{~h}$ at room temperature, so it was stored at $-23^{\circ} \mathrm{C}$ under argon, which increased stability to 2 weeks.

\section{Method B: Synthesis of 3c}




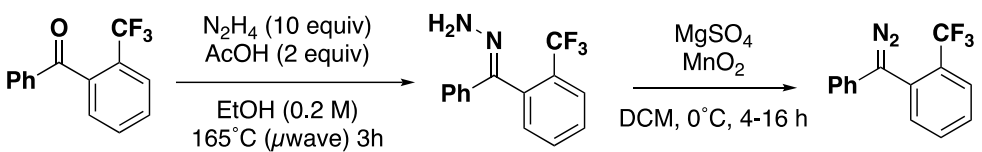

A 20-mL microwave vial with a stir bar was flamed dried, allowed to cool, then sealed. With an Ar balloon to equilibrate pressure, the vial was charged with 2-trifluoromethylbenzophenone in $10 \mathrm{~mL}$ EtOH (4 mmol, $0.4 \mathrm{M})$. Hydrazine ( $40 \mathrm{mmol}, 1.28 \mathrm{~mL}$ ), followed by AcOH $(5 \mathrm{mmol}, 286 \mu \mathrm{L})$ were added, and the solution was sparged with Ar for $5 \mathrm{~min}$. The microwave vial was heated in a microwave reactor to $165{ }^{\circ} \mathrm{C}$ for $3 \mathrm{~h}$, with $15 \mathrm{sec}$ of pre-stirring. The reaction was allowed to cool to room temperature, and the solvent was removed via rotary evaporator. The resulting slurry was dissolved in $\mathrm{Et}_{2} \mathrm{O}(20 \mathrm{~mL})$, washed with $\mathrm{H}_{2} \mathrm{O}(10 \mathrm{~mL})$, brine $(10 \mathrm{~mL})$, and dried with $\mathrm{Na}_{2} \mathrm{SO}_{4}$. The solution was filtered, and the solvent was removed via rotary evaporator. The crude hydrazone mixture was transferred into a flame dried round bottom flask purged with argon and charged with a stir bar. DCM (0.5M) was added, followed by $\mathrm{MgSO}_{4}(100 \mathrm{mg} / \mathrm{mmol})$. The solution was cooled down to $0^{\circ} \mathrm{C}$, and $\mathrm{MnO}_{2}(32 \mathrm{mmol}, 2.78 \mathrm{~g})$ was added in one portion. The solution was monitored by TLC ( $7: 3$ hexanes/ EtOAc) until all hydrazone was consumed. The solution was then filtered through celite, washed with DCM $(3 \times 10 \mathrm{~mL})$, and the solvent was removed via rotary evaporator. Diazo products were purified by column chromatography using (99:1 hexanes: $\mathrm{Et}_{3} \mathrm{~N}$ ) on basic alumina to furnish diazo $3 \mathbf{c}$ as a red liquid in $44 \%$ yield $(461.5 \mathrm{mg})$ over 2 steps.

\section{Method C: Synthesis of aryl-alkyl silanes using Grignards}

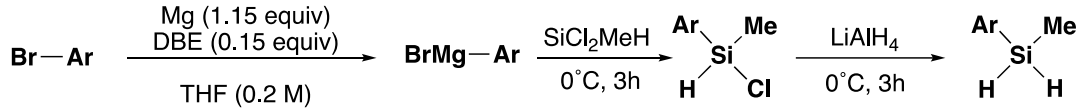

Magnesium turnings (1.15 equiv) were added to THF in an argon-purged 2-neck flask followed by the addition of DBE ( 0.15 equiv). The solution stirred for $1 \mathrm{~h}$ and turned black. The aryl bromide (1.0 equiv) was slowly added dropwise over a 20-minute period, and the solution was brought to reflux for up to $16 \mathrm{~h}$, or until magnesium was no longer observed in the flask. The reaction was allowed to cool to room temperature and stirred an additional 15 minutes. The reaction was cooled to $0{ }^{\circ} \mathrm{C}$ with vigorous stirring, and an alkyldichlorosilane was added quickly in one portion, and stirred for an additional 2-3 hours with no further cooling. The reaction was cooled to $0^{\circ} \mathrm{C}$ again, and $\mathrm{LiAlH}_{4}$ (4.0 M solution in $\mathrm{Et}_{2} \mathrm{O}, 1.0$ equiv) was added dropwise over a 5-minute period and stirred for an additional 2 $\mathrm{h}$. The reaction was slowly quenched with the addition of saturated aq. Rochelle's salt, and filtered over celite. The organic layer was separated and the aqueous layer was washed with $\mathrm{Et}_{2} \mathrm{O}$, then the organic layers were combined and washed with brine, dried with $\mathrm{MgSO}_{4}$, filtered, and then concentrated in vacuo. The products were purified either by bulb-to-bulb distillation via kugelrohr, sublimation, or column chromatography in hexanes.

\section{Method D: Synthesis of aryl-alkyl silanes using lithiates}

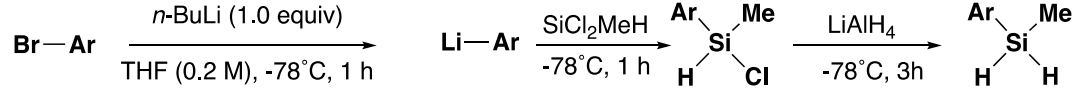

A round-bottom flask with stir bar was charged with an aryl bromide (1.0 equiv), and THF. The reaction was cooled to $-78{ }^{\circ} \mathrm{C}$ with vigorous stirring, and $n$ - $\mathrm{BuLi}(2.5 \mathrm{M}$ solution in hexanes, 1.0 equiv) was added dropwise, and the reaction stirred for $1 \mathrm{~h}$. The alkyldichlorosilane (1.0 equiv) was added quickly in one portion and stirred for an additional $1 \mathrm{~h}$ at $-78^{\circ} \mathrm{C}$. $\mathrm{LiAlH}_{4}\left(4.0 \mathrm{M}\right.$ solution in $\mathrm{Et}_{2} \mathrm{O}, 1.0$ equiv) was added dropwise over a 5 minute period and stirred for an additional $2 \mathrm{~h}$. The reaction was slowly quenched with the addition of saturated aq. Rochelle's salt, and filtered over celite. The organic layer was separated and the aqueous layer was washed with $\mathrm{Et}_{2} \mathrm{O}$, then the organic layers were combined and washed with brine, dried with $\mathrm{MgSO}_{4}$, filtered, and then concentrated in vacuo. The products were purified either by bulb-to-bulb distillation via kugelrohr, sublimation, or column chromatography in hexanes to furnish pure silanes.

\section{Method E: Synthesis of 1m}




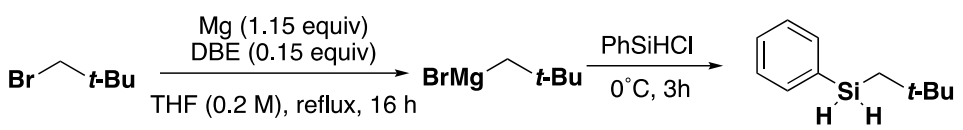

Magnesium turnings ( 1.15 equiv, $5.75 \mathrm{mmol}, 140 \mathrm{mg}$ ) were added to an argon-purged, 25-mL 2-neck flask. THF $(10 \mathrm{~mL})$, followed by the addition of DBE ( 0.15 equiv) were added. The solution stirred for $1 \mathrm{~h}$ and turned black. Neopentyl bromide (1.0 equiv, $5.0 \mathrm{mmol}, 630 \mu \mathrm{L}$ ) was added dropwise, and the solution was brought to reflux for up to $16 \mathrm{~h}$. The reaction was allowed to cool to room temperature and stirred an additional 15 minutes. The reaction was cooled to $0{ }^{\circ} \mathrm{C}$ with vigorous stirring, and chlorophenylsilane (1.0 equiv, $5.0 \mathrm{mmol}, 666 \mu \mathrm{L}$ ) was added quickly in one portion and stirred for an additional 2-3 hours with no further cooling. The reaction was slowly quenched with the addition of saturated aqueous $\mathrm{NH}_{4} \mathrm{Cl}(10 \mathrm{~mL})$ and filtered over celite. The organic layer was separated and the aqueous layer was washed with $\mathrm{Et}_{2} \mathrm{O}$, then the organic layers were combined and washed with brine, dried with $\mathrm{MgSO}_{4}$, filtered, and then concentrated in vacuo. The crude mixture was purified by column chromatography (100\% hexanes) to furnish silane $1 \mathbf{m}$ as an oil in $25 \%$ yield ( $231 \mathrm{mg}, 1.3 \mathrm{mmol})$.

\section{IIb. Procedures for Enantioselective Si-H insertions (4a-o; 5a-j)}

\section{Method E: Synthesis of racemic standard for HPLC analysis}

A $4 \mathrm{~mL}$ reaction vial equipped with stir bar and $50 \mathrm{mg} 4 \AA$ molecular sieves was heated under flame and dried under high vacuum ( $>1$ torr). After the vial cooled to room temperature, it was purged with argon and $\mathrm{Rh}_{2} \mathrm{OAc}_{4}(0.002$ $\mathrm{mmol})$, silane $(0.5 \mathrm{mmol})$. The vial was re-purged with argon, and $1 \mathrm{~mL}$ of DCM was added. A diazo compound $(0.1$ $\mathrm{mmol}$ ) was weighed into a separate flame-dried vial, and $1 \mathrm{~mL}$ of DCM was added, then drawn into a syringe. Using a long needle, the syringe was placed on a syringe pump with the needle in the stirring solution. The syringe pump was program to add the solution over a period of 1 hour at room temperature. After $1 \mathrm{~h}$, the solution was diluted with $5 \mathrm{~mL}$ of hexane, and filtered through celite, and concentrated in vacuo. The presence of insertion product was verified by 1 H NMR analysis, and HPLC analysis was then conducted.

\section{Method F: General procedure for enantioselective dirhodium(II)-catalyzed insertion with donor/donor carbenes (prochiral and symmetrical)}

A $8 \mathrm{~mL}$ reaction vial equipped with stir bar and $100 \mathrm{mg} 4 \AA$ molecular sieves was heated under flame and dried under high vacuum ( $>1$ torr). After the vial cooled to room temperature, it was purged with argon, followed by addition of $\mathrm{Rh}_{2}(S$-TCPTTL $) 4(0.002 \mathrm{mmol}, 3.6 \mathrm{mg})$ and silane $1 \mathrm{a}(1 \mathrm{mmol}, 137 \mu \mathrm{L})$. The vial was re-purged with argon, and 2 $\mathrm{mL}$ of PhMe was added. Diazo compound $3 \mathbf{a}(0.2 \mathrm{mmol}, 39.0 \mathrm{mg}))$ was weighed into a separate flame-dried vial, and $2 \mathrm{~mL}$ of PhMe was added, then drawn into a syringe. Using a long needle, the syringe was placed on a syringe pump with the needle in the stirring solution. The syringe pump was programed to add the solution over a period of $1 \mathrm{~h}$ at room temperature. 

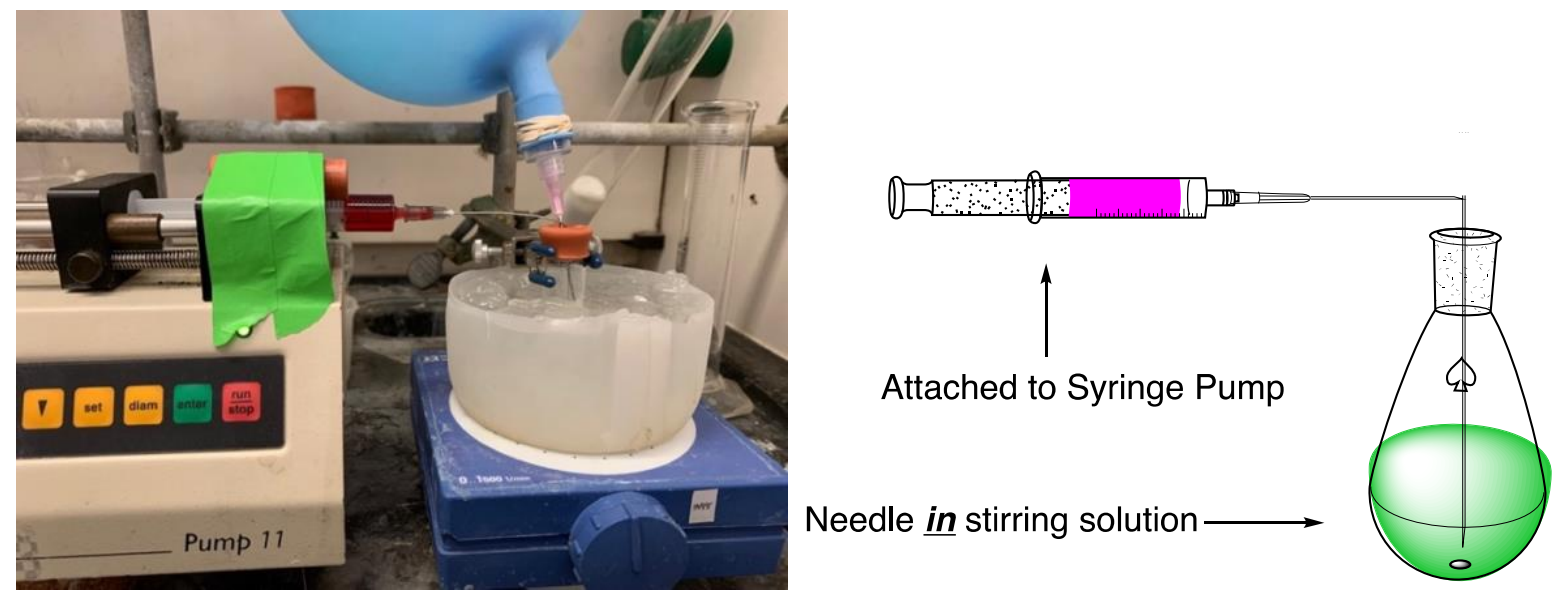

Figure S1. Example insertion with diagram

Thirty minutes after diazo addition, the solution was diluted with $5 \mathrm{~mL}$ of hexane, and filtered through celite, and concentrated in vacuo. The crude mixture was diluted in hexanes and ran through a short silica plug $(4 \mathrm{~cm}$ in a Pasteur pipette) to remove catalyst, and concentrated in vacuo. The crude mixture was analyzed by NMR to determine dr, via the methyl resonance off the benzene ring. The product was then purified using column chromatography (dry loaded sample in silica, 98:2 hexanes/DCM) to furnish silane 5a as an oil in 91\% yield (55.0 mg, $0.18 \mathrm{mmol}, 93: 7 \mathrm{dr}$, 93:7 er).

\section{Method G: Procedure for gram-scale enantioselective dirhodium(II)-catalyzed insertion of diazo 3a and methylphenylsilane 1a}

A $100 \mathrm{~mL}$ round-bottom flask equipped with stir bar and $2.50 \mathrm{~g}$ of $4 \AA$ molecular sieves was heated in the oven for $24 \mathrm{~h}$ and dried under high vacuum (>1 torr). After the flask cooled to room temperature, $\mathrm{Rh}_{2}(S$-TCPTTL) $4(0.0025$ mmol, $5.1 \mathrm{mg})$ and methylphenylsilane $1 \mathrm{a}(25.5 \mathrm{mmol}, 3.50 \mathrm{~mL})$ were added. The flask was purged with Ar, followed by addition of PhMe $(25 \mathrm{~mL})$. Diazo compound 3a $(5.1 \mathrm{mmol}, 1.00 \mathrm{~g})$ was weighed into a separate flame-dried vial, and $25 \mathrm{~mL}$ of PhMe was added, then drawn into a $30-\mathrm{mL}$ syringe. Using a long needle, the syringe was placed on a syringe pump with the needle in the stirring solution. The syringe pump was programed to add the solution over a period of $2.5 \mathrm{~h}$ at room temperature. 


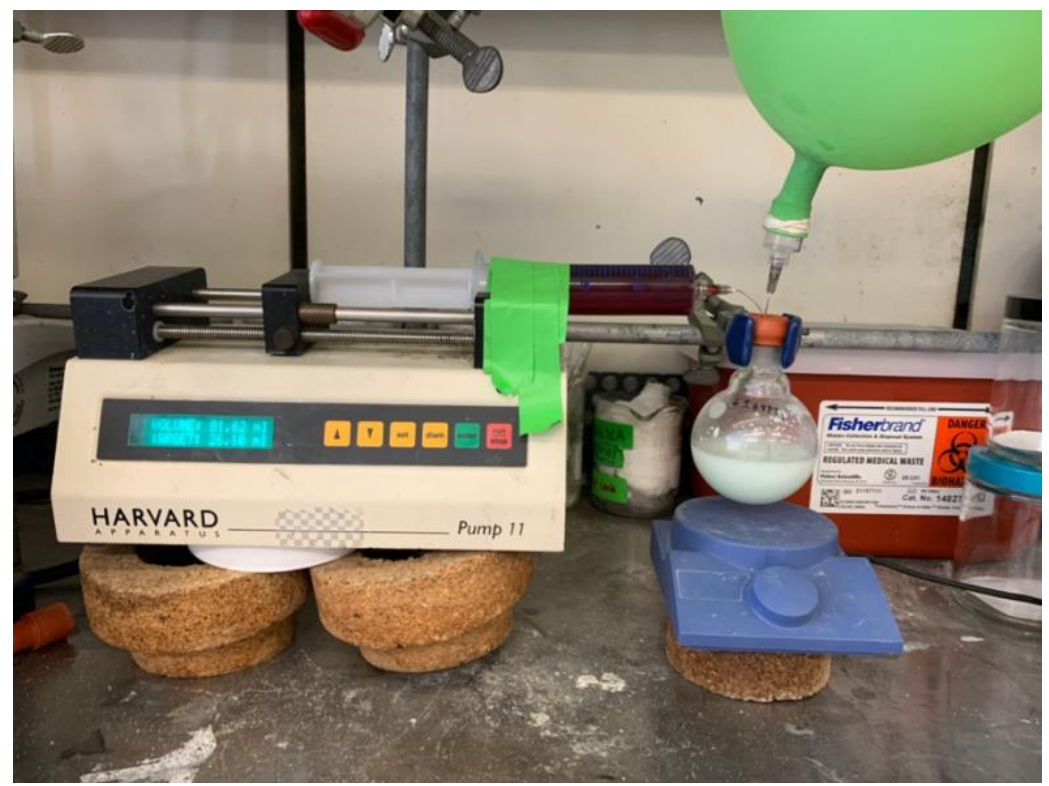

Figure S2. Gram-scale insertion

After $2.5 \mathrm{~h}$, the solution was filtered, and concentrated in vacuo. The crude mixture was analyzed by NMR to determine $\mathrm{dr}$, via the methyl resonance off the benzene ring. The product was then purified using column chromatography (dry loaded sample in silica, 98:2 hexanes/DCM) to furnish silane 5a as an oil in 89\% yield (93:7 dr, $1.34 \mathrm{~g}, 4.5 \mathrm{mmol})$.

Silane 5a was found to be stable to air and atmospheric moisture for $>3$ weeks with minimal decomposition and no loss of dr or er.

\section{IIc. Procedures for HPLC samples}

\section{Method H: General procedure for hydrolysis for HPLC Analysis}

Silane was added to a $4 \mathrm{~mL}$ reaction vial, $(10 \mathrm{mg})$ followed by $\mathrm{THF} / \mathrm{H}_{2} \mathrm{O}(99: 1 \mathrm{v} / \mathrm{v}, 0.5 \mathrm{~mL})$, then $\mathrm{Pd} / \mathrm{C}(10 \mathrm{mg})$ (preactivated with $\mathrm{PhSiH}_{3}$ ). The reaction was monitored by TLC until full consumption of the silane was observed. The reaction was filtered through celite and concentrated in vacuo. DCM $(0.1 \mathrm{~mL})$ was added, and the sample was spotted onto a TLC plate $(5 \times 5 \mathrm{~cm})$. The plate was placed in a TLC chamber to develop, and the silanol was etched off the plate with a razor blade. The loose silica was washed with 70:30 hexanes:IPA $(1.0 \mathrm{~mL})$ and filtered through celite to furnish HPLC samples. 


\section{IId. Procedures for Functionalization}

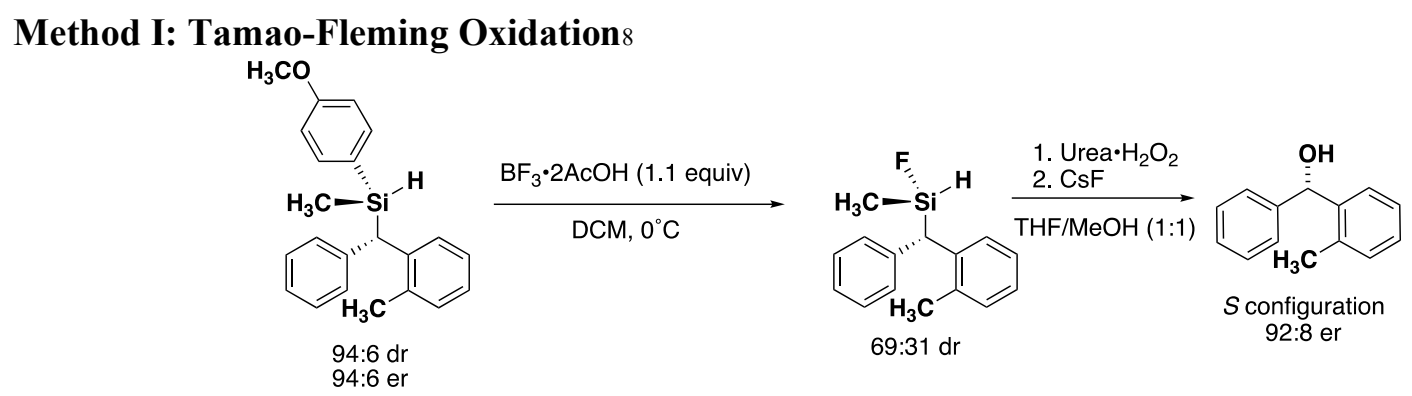

To a flame-dried, Ar-purged 8-mL reaction vial charged with stir bar, silane 5e (258 $\mathrm{mg}, 0.77 \mathrm{mmol})$ was dissolved in DCM $(3 \mathrm{~mL})$ and added to the vial. The vial was cooled to $0^{\circ} \mathrm{C}$, and $\mathrm{BF}_{3} \cdot 2 \mathrm{AcOH}(120 \mathrm{ul}, 0.85 \mathrm{mmol})$ was added to the vial while stirring. The conversion of the arylsilane to the silyl-fluoride was monitored using TLC (9:1 hexanes/EtOAc). Once the reaction was complete the reaction was diluted with $10 \mathrm{~mL}$ of DCM. Saturated aqueous $\mathrm{NaHCO}_{3}$ solution $(8 \mathrm{~mL})$ was added and stirred until the evolution of gas ceased completely. The organic layer was separated, collected, and the aqueous layer was extracted with $\mathrm{CH}_{2} \mathrm{Cl}_{2}(2 \times 15 \mathrm{~mL})$. The organic layer was dried over $\mathrm{Na}_{2} \mathrm{SO}_{4}$ and concentrated in vacuo to afford a silyl-fluoride intermediate that could be isolated as a clear viscous oil and was identified using ${ }_{1} \mathrm{H}$ NMR and ${ }_{19} \mathrm{~F}$ NMR spectroscopy. The silyl-fluoride was not purified and was carried forward directly into the next step. The crude mixture was dissolved in 1:1 MeOH:THF (5 mL), and transferred to a flame-dried flask charged with a stir bar. Next, urea hydrogen peroxide was added $(80.0 \mathrm{mg}, 0.85 \mathrm{mmol})$, and the reaction was monitored by TLC until full consumption of the silyl fluoride was observed. Lastly, CsF was added (128.4 $\mathrm{mg}, 0.85 \mathrm{mmol}$ ), and the reaction was monitored by TLC until benzhydrol 12 was observed (based on TLC). The reaction mixture was filtered though celtie, washed with $\mathrm{H} 2 \mathrm{O}(5 \mathrm{~mL})$, and dried over $\mathrm{Na} 2 \mathrm{SO} 4$. The solution was filtered and concentrated in vacuo to furnish crude benzhydrol 12. An HPLC sample of the reaction mixture was prepared and analyzed using CSP-HPLC (92:8 er); when compared to literature values, they matched with previously reported data with the $S$-configuration.

\section{Method J: C-H Silylation9}

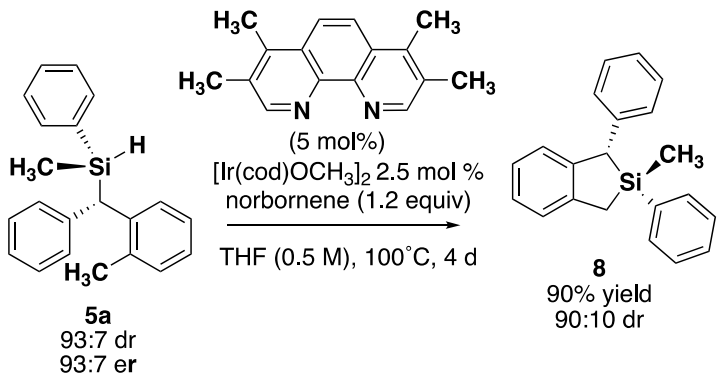

To a flame-dried, 4-mL reaction vial charged with stir bar, silane $\mathbf{5 a}(100 \mathrm{mg}, 0.33 \mathrm{mmol})$, norbornene, 3,4,7,8tetramethyl-1,10-phenthroline (2.0 mg, $0.0084 \mathrm{mmol})$, [Ir(cod)OMe]2, $(5.5 \mathrm{mg}, 0.084 \mathrm{mmol})$ was purged with argon, then dissolved in THF $(0.7 \mathrm{~mL})$ and screwed tightly. The vial was heated to $100^{\circ} \mathrm{C}$ and stirred for 4 days. The reaction was allowed to cool to room temperature, then diluted with hexanes $(10 \mathrm{~mL})$, and filtered through a pad of celite. The organic solution was concentrated in vacuo, and the product was purified using column chromatography (98:2 hexanes/DCM) to furnish silane $\mathbf{1 1}$ as an oil in $90 \%$ yield $(89.2 \mathrm{mg}, 90: 10 \mathrm{dr})$. 


\section{Method K: General Procedure for Pd/C Hydrolysis}
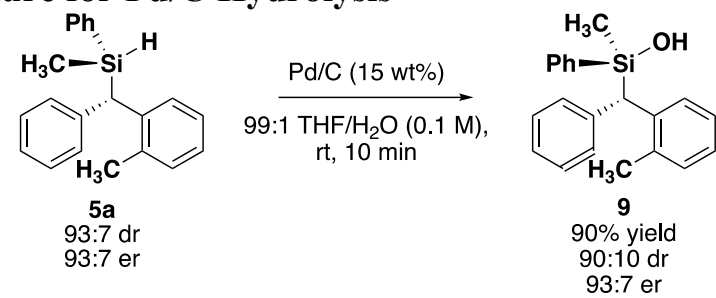

To a 4-mL reaction vial charged with stir bar, silane $5 \mathbf{5}(100 \mathrm{mg}, 0.33 \mathrm{mmol}, 93: 7 \mathrm{dr}$; $93: 7 \mathrm{er}), \mathrm{Pd} / \mathrm{C}$ (15.0 mg, 15 wt $\%$ ) was purged with argon, then dissolved in THF (containing 1\% v/v $\mathrm{H}_{2} \mathrm{O}$ ). The consumption of starting material was monitored by TLC, about $10 \mathrm{~min}$. Once fully consumed, DCM $(5 \mathrm{~mL})$ was added, and the reaction was filtered through a thin pad of celite. The solution was dried with $\mathrm{Na}_{2} \mathrm{SO}_{4}$, filtered, and concentrated in vacuo. The product was purified using column chromatography (95:5 hexanes/EtOAc) to furnish silanol $\mathbf{9}$ as an oil in $90 \%$ yield $(94.6 \mathrm{mg}$, 90:10 dr, 93:7 er).

\section{Method L: Dehydro-coupling reaction}
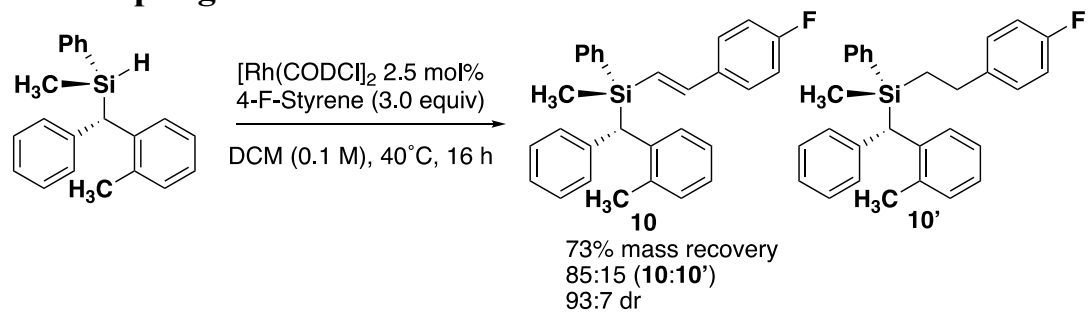

To a flame-dried, 8-mL reaction vial charged with stir bar, silane 5a (104 mg, 0.34 mmol, 93:7 dr; 93:7 er), 4fluorostyrene $(19 \mu \mathrm{L}, 1.0 \mathrm{mmol})$ was purged with argon, then dissolved in DCM $(3.5 \mathrm{~mL})$. After $5 \mathrm{~min},[\mathrm{Rh}(\mathrm{COD}) \mathrm{Cl}]_{2}$ ( 0.03 equiv, $5.2 \mathrm{mg}, 0.01 \mathrm{mmol}$ ) was added, and the solution turned yellow, and was heated to $40^{\circ} \mathrm{C}$ and stirred for 16 $\mathrm{h}$. The reaction was diluted with hexanes and filtered through a thin silica pad to remove catalyst. The solvent was removed via rotary evaporator, and the product was purified using column chromatography $(98: 2 \rightarrow 90: 10$ hexanes/DCM) to furnish silane $\mathbf{1 0}$ as an oil in 62\% yield (73\% mass recovery (104.1 mg); 85:15 mixture with hydrosilylation product 10', 93:7 dr by ${ }_{19} \mathrm{~F}$ NMR). The determination of 85:15 was confirmed using ${ }_{1} \mathrm{H}$ NMR Spectroscopy by comparing the relative integrals of the peaks at 4.10 and $3.97 \mathrm{ppm}$. 


\section{Analysis and Optimization}

\section{IIIa. Optimization of enantioselective donor/donor carbene insertion of prochiral silanes}

Table S1. Optimization of Enantioselective donor/donor insertion into prochiral silanes

\begin{tabular}{|c|c|c|c|c|c|c|c|c|c|}
\hline & & $\begin{array}{c}\mathrm{Ph}, \mathrm{C} \\
\mathrm{Hi}^{\prime} \mathrm{Si}^{\mathrm{H}} \\
1 \mathrm{H} \\
5 \text { equiv }\end{array}$ & $\begin{array}{l}\text { 2a; } \mathrm{R}=\mathrm{H}^{2} \\
\text { Ia; } \mathrm{R}=\mathrm{CH}_{3} \\
\text { Inverse addition }\end{array}$ & $\begin{array}{c}1 \mathrm{~mol} \% \mathrm{Rh}_{2} \mathrm{~L}_{4} \\
\mathrm{DCM}(0.05 \mathrm{M}), 4 \AA \AA \mathrm{M} \\
23^{\circ} \mathrm{C}, 1.5 \mathrm{~h}\end{array}$ & $\begin{array}{r}\quad \mathrm{Ph} \\
\mathrm{H}_{3} \mathrm{C}- \\
\mathrm{IS} \quad \mathrm{Ph} \\
4 \mathrm{a} ; \mathrm{R}=\mathrm{H}\end{array}$ & $5 \mathrm{a} ; \mathrm{R}=\mathrm{CH}_{3}$ & & & \\
\hline Entry & $\mathrm{R}$ & Catalyst & $\%$ loading & Solvent & Temp & additive & $\%$ yield $a$ & $\mathrm{dr}_{b}$ & $\mathrm{er}_{c}$ \\
\hline 1 & $\mathrm{H}$ & $\mathrm{Cu}(\mathrm{OTf})_{2}$ & 10 & $\mathrm{DCM}$ & rt & - & $<5$ & - & - \\
\hline 2 & $\mathrm{H}$ & $\mathrm{Cu}[\mathrm{MeCN}]_{4} \mathrm{PF}_{6}$ & 10 & $\mathrm{DCM}$ & rt & - & $<5$ & - & - \\
\hline 3 & $\mathrm{H}$ & $(\operatorname{Ir}[\mathrm{COD}] \mathrm{Cl})_{2}$ & 5 & DCM & rt & - & $<5$ & - & - \\
\hline 4 & $\mathrm{H}$ & {$[\mathrm{IrCpCl}]_{2}$} & 5 & DCM & rt & - & $<5$ & - & - \\
\hline 5 & $\mathrm{H}$ & {$[\mathrm{Ru}(\mathrm{p} \text {-cymeme }) \mathrm{Cl}]_{2}$} & 5 & $\mathrm{DCM}$ & $\mathrm{rt}$ & - & $<5$ & - & - \\
\hline 6 & $\mathrm{H}$ & $\mathrm{Fe}(\mathrm{OTf})_{2}$ & 20 & DCM & rt & - & $<5$ & - & - \\
\hline 7 & $\mathrm{H}$ & $\mathrm{Rh}_{2} \mathrm{OAc}_{4}$ & 1 & $\mathrm{DCM}$ & rt & - & 35 & - & $50: 50$ \\
\hline 8 & $\mathrm{H}$ & $\mathrm{Rh}_{2}(R \text {-PTAD })_{4}$ & 1 & DCM & $\mathrm{rt}$ & - & 72 & - & $61: 39$ \\
\hline 9 & $\mathrm{H}$ & $\mathrm{Rh}_{2}(\mathrm{~S}-\mathrm{PTTL})_{4}$ & 1 & DCM & $\mathrm{rt}$ & - & 62 & - & $64: 36$ \\
\hline 10 & $\mathrm{H}$ & $\mathrm{Rh}_{2}(\mathrm{~S}-\mathrm{BPTTL})_{4}$ & 1 & DCM & $\mathrm{rt}$ & - & 62 & - & $64: 36$ \\
\hline 11 & $\mathrm{H}$ & $\mathrm{Rh}_{2}(\mathrm{~S}-\mathrm{PTV})_{4}$ & 1 & DCM & rt & - & 67 & - & $59: 41$ \\
\hline 12 & $\mathrm{H}$ & $\mathrm{Rh}_{2}(\mathrm{~S}-\mathrm{DOSP})_{4}$ & 1 & DCM & $\mathrm{rt}$ & - & 64 & - & $55: 45$ \\
\hline 13 & $\mathrm{H}$ & $\mathrm{Rh}_{2}(S \text {-BTPCP })_{4}$ & 1 & $\mathrm{DCM}$ & rt & - & $<5$ & - & ND \\
\hline 14 & $\mathrm{H}$ & $\mathrm{Rh}_{2}(5 R \text {-MEPY })_{4}$ & 1 & DCM & rt & - & $<5$ & - & ND \\
\hline 15 & $\mathrm{H}$ & $\mathrm{Rh}_{2}(\mathrm{~S}-\mathrm{TCPTTL})_{4}$ & 1 & DCM & $\mathrm{rt}$ & $4 \AA \mathrm{MS}_{\mathrm{d}}$ & 76 & - & $76: 24$ \\
\hline 16 & $\mathrm{H}$ & $\mathrm{Rh}_{2}(\mathrm{~S}-\mathrm{TCPTTL})_{4}$ & 3 & Heptane & $\mathrm{rt}$ & $4 \AA \mathrm{MS}_{\mathrm{d}}$ & 78 & - & $82: 18$ \\
\hline 17 & $\mathrm{H}$ & $\mathrm{Rh}_{2}(\mathrm{~S}-\mathrm{TCPTTL})_{4}$ & 3 & Benzene & $\mathrm{rt}$ & $4 \AA \mathrm{MS}_{\mathrm{d}}$ & 74 & - & $82: 18$ \\
\hline 18 & $\mathrm{H}$ & $\mathrm{Rh}_{2}(\mathrm{~S}-\mathrm{TCPTTL})_{4}$ & 3 & Cyclohexane & $\mathrm{rt}$ & $4 \AA \mathrm{MS}_{\mathrm{d}}$ & 74 & - & $82: 18$ \\
\hline 19 & $\mathrm{H}$ & $\mathrm{Rh}_{2}(\mathrm{~S}-\mathrm{TCPTTL})_{4}$ & 3 & $\mathrm{PhMe}$ & $\mathrm{rt}$ & $4 \AA \mathrm{MS}_{\mathrm{e}}$ & 78 & - & $82: 18$ \\
\hline 20 & $\mathrm{CH}_{3}$ & $\mathrm{Rh}_{2} \mathrm{OAc}_{4}$ & 1 & $\mathrm{PhMe}$ & $\mathrm{rt}$ & $4 \AA \mathrm{MS}_{\mathrm{e}}$ & 45 & $55: 45$ & $50: 50$ \\
\hline 21 & $\mathrm{CH}_{3}$ & $\mathrm{Rh}_{2} \mathrm{OAc}_{4}$ & 1 & $\mathrm{PhMe}$ & $\mathrm{rt}$ & $4 \AA \mathrm{MS}_{\mathrm{e}}$ & 45 & $55: 45$ & $50: 50$ \\
\hline 22 & $\mathrm{CH}_{3}$ & $\mathrm{Rh}_{2}(R \text {-PTAD })_{4}$ & 1 & $\mathrm{PhMe}$ & $\mathrm{rt}$ & $4 \AA \mathrm{MS}_{\mathrm{e}}$ & 75 & $63: 37$ & ND \\
\hline 24 & $\mathrm{CH}_{3}$ & $\mathrm{Rh}_{2}(\mathrm{~S}-\mathrm{DOSP})_{4}$ & 1 & $\mathrm{PhMe}$ & $\mathrm{rt}$ & $4 \AA \mathrm{MS}_{\mathrm{e}}$ & 70 & $61: 39$ & ND \\
\hline 25 & $\mathrm{CH}_{3}$ & $\mathrm{Rh}_{2}(S \text {-BTPCP })_{4}$ & 1 & $\mathrm{PhMe}$ & $\mathrm{rt}$ & $4 \AA \mathrm{MS}_{\mathrm{e}}$ & $<5$ & - & ND \\
\hline 26 & $\mathrm{CH}_{3}$ & $\mathrm{Rh}_{2}(\mathrm{~S}-\mathrm{TCPTTL})_{4}$ & 1 & $\mathrm{PhMe}$ & $\mathrm{rt}$ & $4 \AA \mathrm{MS}_{\mathrm{e}}$ & 91 & $92: 8$ & $93: 7$ \\
\hline 27 & $\mathrm{CH}_{3}$ & $\mathrm{Rh}_{2}(\mathrm{~S}-\mathrm{TCPTTL})_{4}$ & 0.1 & $\mathrm{PhMe}$ & $\mathrm{rt}$ & $4 \AA \mathrm{MS}_{\mathrm{e}}$ & 85 & $93: 7$ & $93: 7$ \\
\hline 28 & $\mathrm{CH}_{3}$ & $\mathrm{Rh}_{2}(\mathrm{~S}-\mathrm{TCPTTL})_{4}$ & 0.01 & $\mathrm{PhMe}$ & $\mathrm{rt}$ & $4 \AA \mathrm{MS}_{\mathrm{e}}$ & 88 & $93: 7$ & $93.5: 6.5$ \\
\hline 29 & $\mathrm{CH}_{3}$ & $\mathrm{Rh}_{2}(\mathrm{~S}-\mathrm{TCPTTL})_{4}$ & 0.001 & $\mathrm{PhMe}$ & $\mathrm{rt}$ & $4 \AA \mathrm{MS}_{\mathrm{e}}$ & 32 & $93: 7$ & $93.5: 6.5$ \\
\hline $30_{f}$ & $\mathrm{CH}_{3}$ & $\mathrm{Rh}_{2}(\mathrm{~S}-\mathrm{TCPTTL})_{4}$ & 0.05 & $\mathrm{PhMe}$ & $\mathrm{rt}$ & $4 \AA \mathrm{MS}_{\mathrm{e}}$ & 89 & $93: 7$ & $93.5: 6.5$ \\
\hline
\end{tabular}




\begin{tabular}{|c|c|c|c|c|c|c|c|c|c|}
\hline Entry & $\mathrm{R}$ & Catalyst & $\%$ loading & Solvent & Temp & additive & $\%$ yield $a$ & $\mathrm{dr} b$ & $\mathrm{er}_{c}$ \\
\hline $31_{g}$ & $\mathrm{CH}_{3}$ & $\mathrm{Rh}_{2}(\mathrm{~S}-\mathrm{TCPTTL})_{4}$ & 1 & $\mathrm{PhMe}$ & $\mathrm{rt}$ & $4 \AA \mathrm{MS}_{\mathrm{e}}$ & 40 & $92: 8$ & $93: 7$ \\
\hline $32 h$ & $\mathrm{CH}_{3}$ & $\mathrm{Rh}_{2}(\mathrm{~S}-\mathrm{TCPTTL})_{4}$ & 1 & $\mathrm{PhMe}$ & $\mathrm{rt}$ & $4 \AA \mathrm{MS}_{\mathrm{e}}$ & 81 & $92: 8$ & $93: 7$ \\
\hline 33 & $\mathrm{CH}_{3}$ & $\mathrm{Rh}_{2}(\mathrm{~S}-\mathrm{TCPTTL}) 4$ & 1 & $\mathrm{PhMe}$ & $0^{\circ} \mathrm{C}$ & $4 \AA \mathrm{MS}_{\mathrm{e}}$ & 78 & $93: 7$ & $93: 7$ \\
\hline 34 & $\mathrm{CH}_{3}$ & $\mathrm{Rh}_{2}(\mathrm{~S}-\mathrm{TCPTTL})_{4}$ & 1 & $\mathrm{PhMe}$, & $-30^{\circ} \mathrm{C}$ & $4 \AA \mathrm{MS}_{\mathrm{e}}$ & $<5$ & - & ND \\
\hline 35 & $\mathrm{CH}_{3}$ & $\mathrm{Rh}_{2}(\mathrm{~S}-\mathrm{TCPTTL}) 4$ & 1 & $\mathrm{PhMe}$, & $-78^{\circ} \mathrm{C}$ & $4 \AA \mathrm{MS}_{\mathrm{e}}$ & $<5$ & - & ND \\
\hline
\end{tabular}

a Determined using $1 \mathrm{H}$ NMR a1nalysis using PhTMS as an internal standard. $b$ Determined using $1 \mathrm{H}$ NMR analysis of isolated product prior to purification. ${ }_{c}$ Determined using CSP-HPLC after Pd/C hydrolysis. $d$ MS were heated under high vacuum ( $>1$ torr) and cooled to rt prior to use. $e$ MS were flame dired and dried under high vacuum ( $>1$ torr). $\mathrm{f}$ Reaction run on gram scale for 3a $(5.1 \mathrm{mmol})$ at $0.1 \mathrm{M}$ PhMe. $g$ Diazo was added in a single portion. $h$ Diazo added dropwise by hand over 5 minutes.

\section{IIIb. Optimization of C-H silylation of Silane 5a}

Table S2. Optimization of C-H silylation

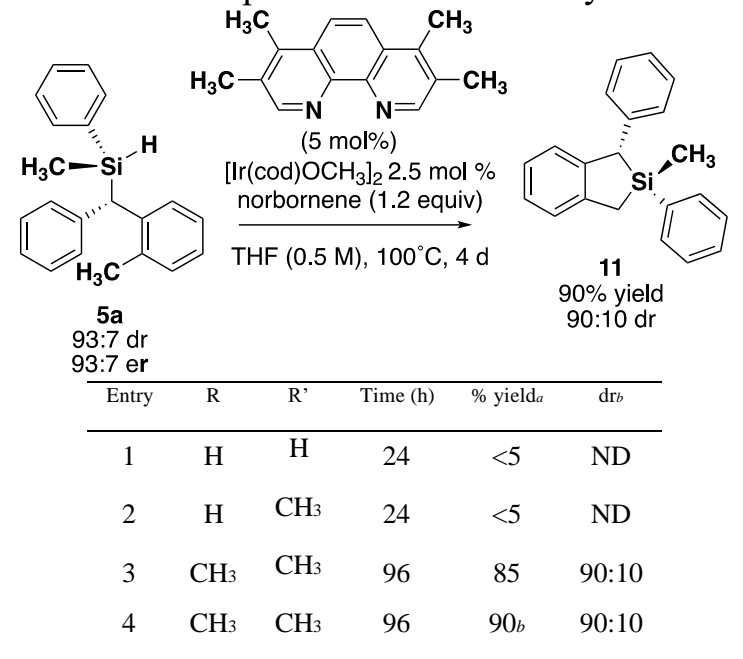

${ }_{a}$ Determined using 1 H NMR analysis using PhTMS as an internal standard, 0.1 mmol silane 5a. $b 0.33$ mmol 5a; isolated yield. 


\section{IIIc. Optimization of dehydrocoupling of Silane 5a}

Table S3. Optimization of dehydrocoupling
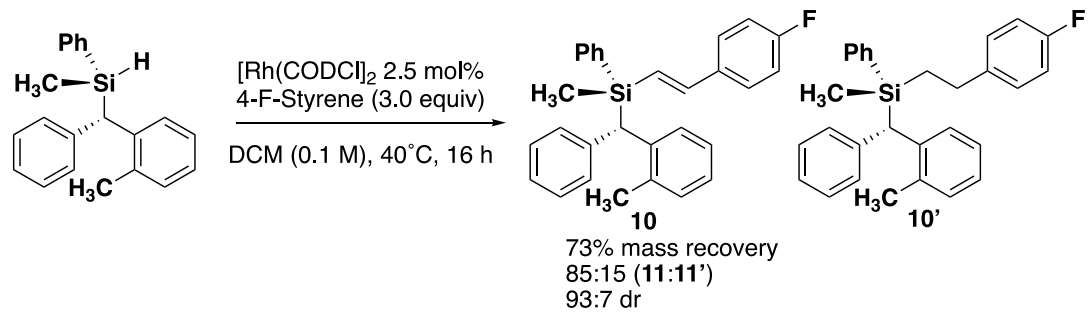

\begin{tabular}{|c|c|c|c|c|c|c|c|c|c|}
\hline entry & $\begin{array}{c}\mathbf{5 a} \\
(\mathrm{mg})\end{array}$ & $\begin{array}{c}\text { Catalyst, \% } \\
\text { loading }\end{array}$ & solvent & time $(\mathrm{h})$ & temp & equiv styrene & $\begin{array}{c}\mathbf{5 a} \\
\text { consumption } \\
(\%) \mathrm{a}\end{array}$ & 10:10’a & $\%$ Mass Recovery \\
\hline 1 & 30 & $\operatorname{Pt}(\mathrm{dvs}), 2.5$ & Pentane & $16 \mathrm{~h}$ & $\mathrm{rt}$ & 1.2 & $>95$ & - & ND \\
\hline 2 & 102 & $\operatorname{Pt}(\mathrm{dvs}), 5$ & DCM & $16 \mathrm{~h}$ & $\mathrm{rt}$ & 1.2 & $>95$ & - & ND \\
\hline 3 & 104 & $\operatorname{Pt}(\mathrm{dvs}), 5$ & DCE & $16 \mathrm{~h}$ & 80 & 1.2 & $>95$ & - & ND \\
\hline 4 & 32 & $\operatorname{Pt}(\mathrm{dvs}), 5$ & DCE & $12 \mathrm{~h}$ & 80 & 1.2 & $>95$ & - & ND \\
\hline 5 & 31 & $\mathrm{Pt} \cdot \mathrm{NHC}_{c}, 10$ & DCE & $12 \mathrm{~h}$ & $\mathrm{rt}$ & 1.2 & 10 & - & ND \\
\hline 6 & 32 & $\mathrm{PtO}_{2}, 10$ & DCE & $12 \mathrm{~h}$ & $\mathrm{rt}$ & 1.2 & $>95$ & - & ND \\
\hline 7 & 16 & {$[\mathrm{Rh}(\mathrm{COD}) \mathrm{Cl}]_{2}, 2.5$} & $\mathrm{DCM}$ & 16 & 40 & 1.2 & $>95$ & $85: 15$ & ND \\
\hline 8 & 102 & {$[\mathrm{Rh}(\mathrm{COD}) \mathrm{Cl}]_{2}, 2.5$} & $\mathrm{DCM}$ & 16 & 40 & 1.2 & $>95$ & $50: 50$ & 75 \\
\hline 9 & 31 & {$[\mathrm{Rh}(\mathrm{COD}) \mathrm{Cl}]_{2}, 2.5$} & DCE & 16 & 80 & 3 & $>95$ & $75: 25$ & 80 \\
\hline 10 & 103 & {$[\mathrm{Rh}(\mathrm{COD}) \mathrm{Cl}]_{2}, 2.5$} & DCM & 16 & 40 & 3 & $>95$ & $85: 15$ & 82 \\
\hline 11 & 33 & {$\left[\mathrm{Rh}\left(\mathrm{PPh}_{3}\right) \mathrm{Cl}\right]$} & DCM & 16 & 40 & 3 & $>95$ & $75: 25$ & ND \\
\hline
\end{tabular}

a,b Determined using ${ }_{1} \mathrm{H}$ NMR Spectroscopy. $c$ Weight percent used 


\section{IIId. Determination of absolute configuration}

Based on the results of the Tamao-Fleming oxidation (vide infra), the $S$ configuration is assigned to the chiral carbon formed in the reaction after HPLC analysis compared to previously reported traces.10

For the assigning stereochemistry at silicon, a $1 \mathrm{H}$ NOESY experiment was conducted on 11, which was synthesized in 90:10 dr. Assuming retention of stereochemistry during the $\mathrm{Si}-\mathrm{H}$ insertion step,11,12 NOE transfer between the methyl group and benzhydryl proton indicates a cis relationship, thereby assigning an $S$ configuration to the silicon for the insertion product.

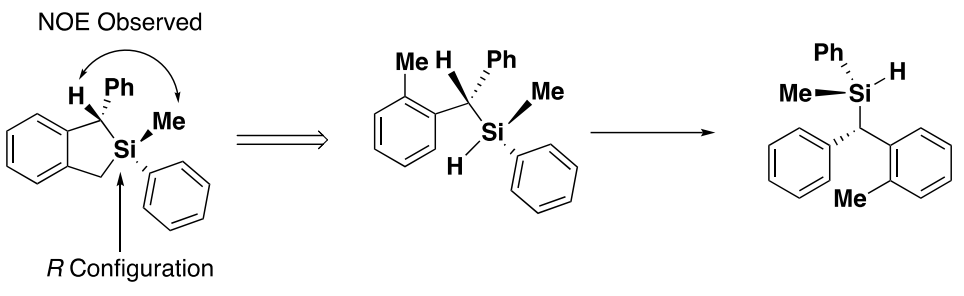

Figure S3. Reasoning for absolute configuration

The stereochemical outcome is applied to all other substrates based on analogy.

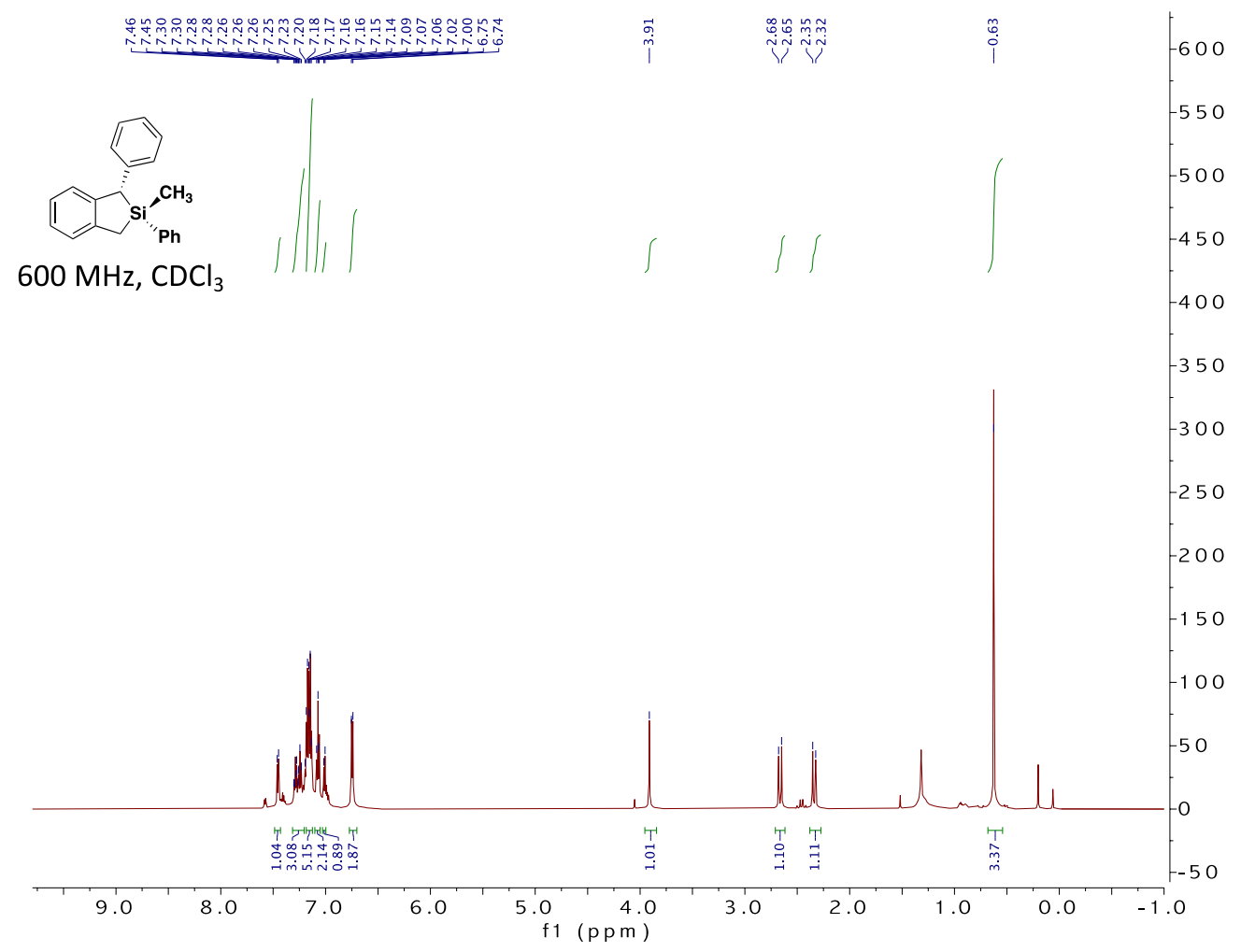

Figure S4. $1 \mathrm{H}$ spectrum of 11. 


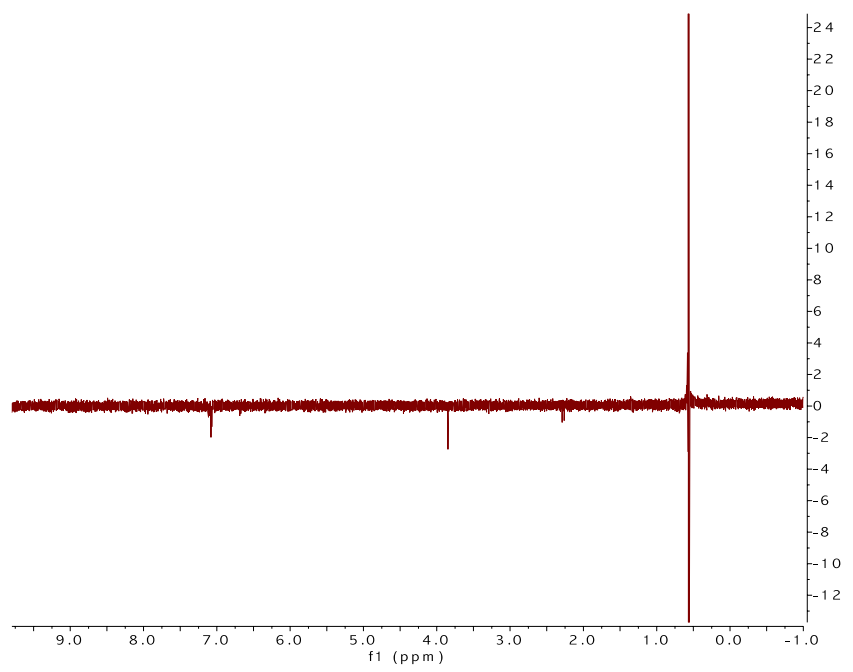

Figure S5. ${ }_{1} \mathrm{H}$ NOESY experiment after $\mathrm{Si}-\mathrm{CH}_{3}$ irradiation.

Transfer was observed to the benzhydryl peak at $\sim 3.8 \mathrm{ppm}$

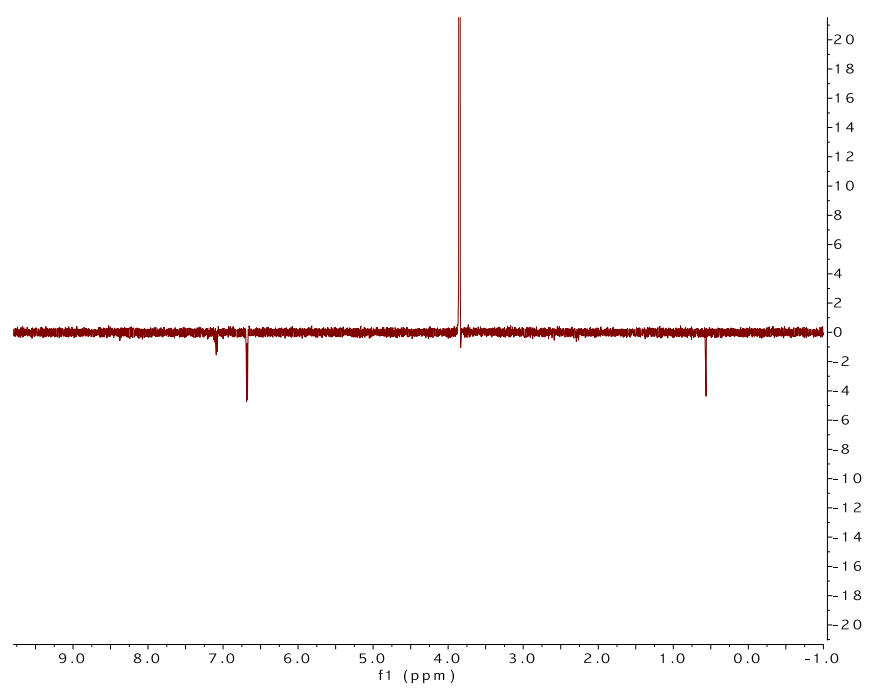

Figure S6. $1 \mathrm{H}$ NOESY experiment after benzyhydryl C-H irradiation. Transfer was observed to the $\mathrm{Si}-\mathrm{CH}_{3}$ irradiation at $\sim 0.5 \mathrm{ppm}$. 


\section{Procedure for in situ ReactIR Analysis (Figure 2)}

A 5-mL microwave reaction vial charged with stir bar, $4 \AA \mathrm{MS}(100 \mathrm{mg})$, then flame-dried and cooled under vacuum ( $>1$ torr). After cooling, the vial was sealed, and PhMe (3mL) was added. A hole was punctured using a spatula, then quickly fitted to the ReactIR probe for background scans. The diazo was added as a solution $(44.0 \mathrm{mg}$ in $1.5 \mathrm{~mL}$ $\mathrm{PhMe})$, and more background scans were taken to identify the diazo on the IR spectrum $(2000-2100 \mathrm{~cm}-1) \cdot \operatorname{Rh}_{2}(S-$ TCPTTL $)_{4}$ was added as a solution in PhMe $(0.5 \mathrm{~mL}$ of $0.3 \mathrm{mg} / \mathrm{mL}$ solution), and the disappearance of diazo $2 \mathbf{a}$ or 3a was monitored to determine a $1_{\text {st }}$ order rate constant $(k)$.

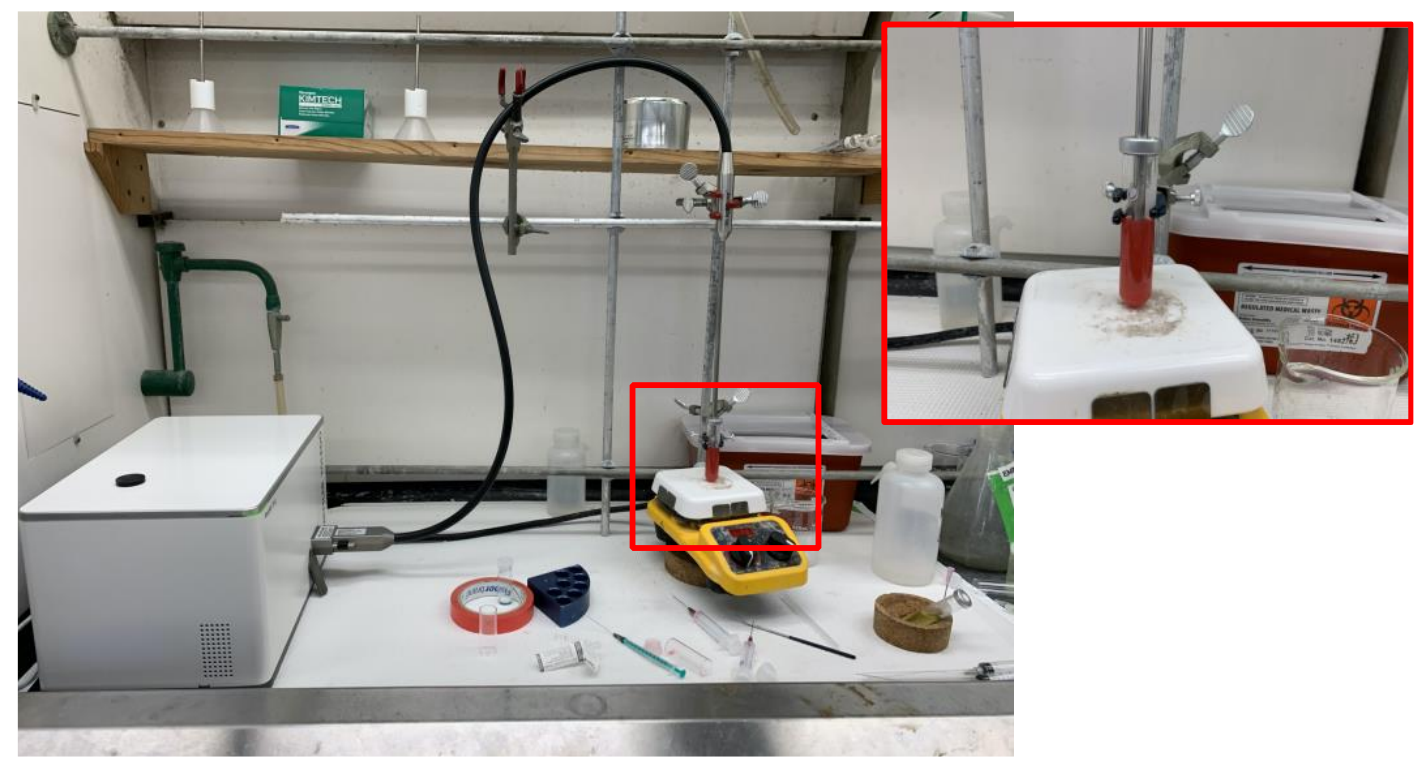

Figure S7. Example of ReactIR setup used for kinetic analysis.

The disappearance of diazo compounds 2a and 3a was monitored at $2041 \mathrm{~cm}-1$ and the trend data was transferred to Microsoft Excel for analysis. The values on the $\mathrm{x}$-axis were manipulated to reflect the total number of minutes after addition of diazo for determination of rate constants. The values on the $\mathrm{Y}$-axis were calculated to conversion by dividing by the maximum absorbance seen. The initial point used for 1 st order rate constant determination was set to when $\mathrm{Rh}_{2}(S \text {-TCPTTL })_{4}$ was added to the reaction vial. For the determination of reaction order, and $\mathrm{R}_{2}$ value, the final point was identified when the concentration plateaued. The reactions were then filtered through a celite plug, and azines $\mathbf{7}$ and $\mathbf{8}$ were characterized by NMR. NMR yields using Ph-TMS were $>90 \%$ for all trials. We then assumed the relative rates of diazo consumption were equivalent to the relative rates of azine formation. 


\section{Trial 1}

A

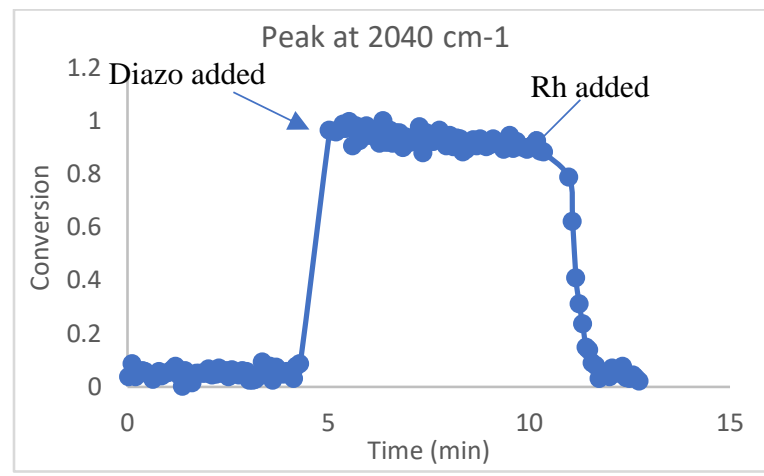

B

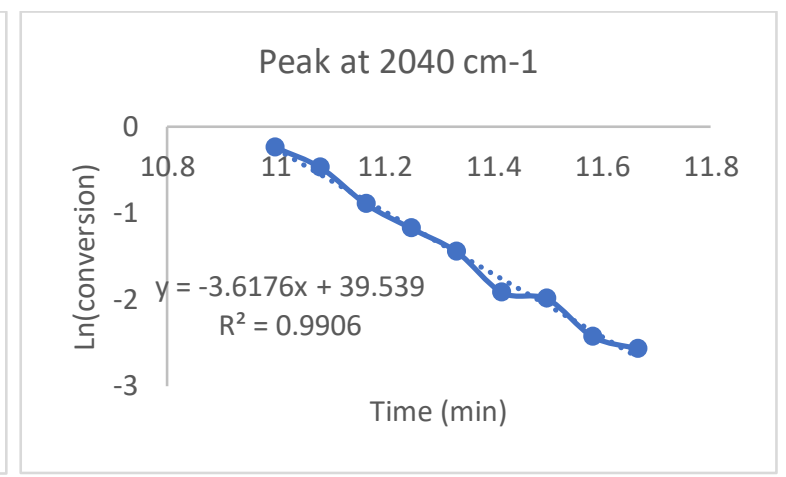

Figure S8. A: Data graphed for reaction with 2a catalyzed by Rh2(S-TCPTTL)4: A) Graph of conversion vs. time. B) Graph of $\ln [$ conv] vs. time once catalyst was added.

Figure S9. ReactIR surface plot for trial 1

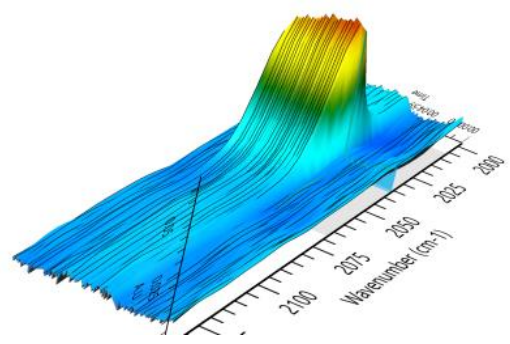

Trial 2

A

B

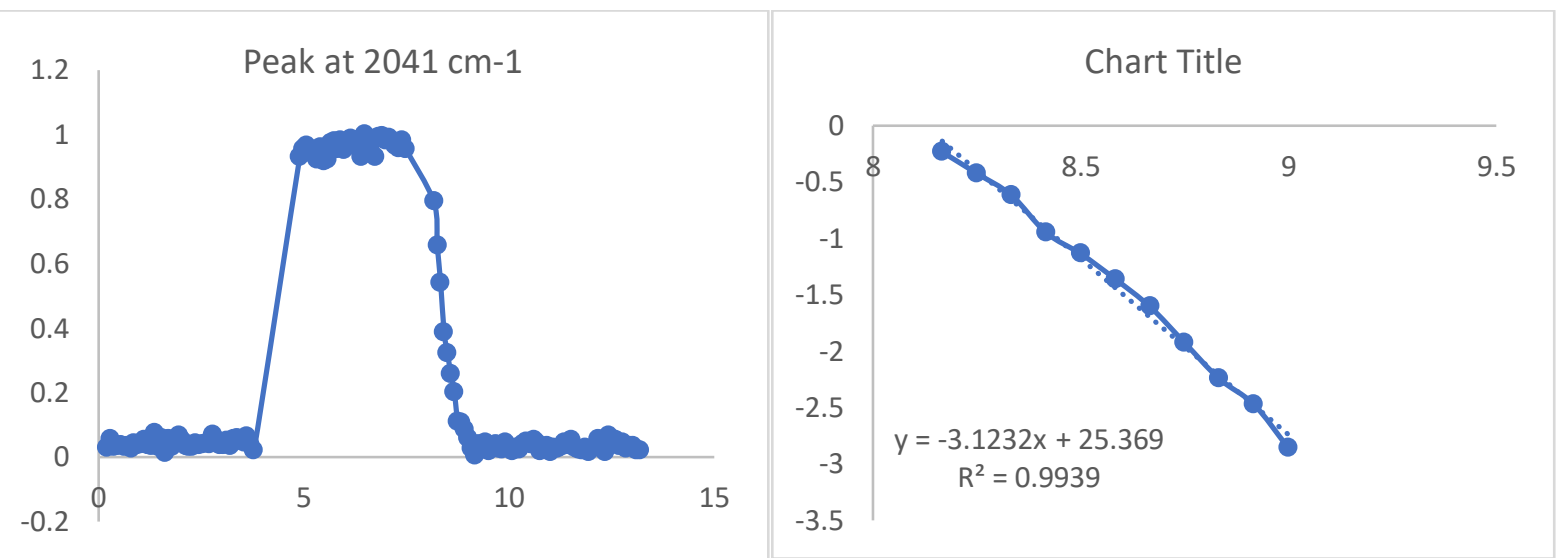

Figure S10. A: Data graphed for reaction with diazo compound 3a catalyzed by $\operatorname{Rh}_{2}(S$-TCPTTL $) 4$ : A) Graph of conversion vs. time. B) Graph of $\ln [$ conv] vs. time once catalyst was added. 
Trial 1

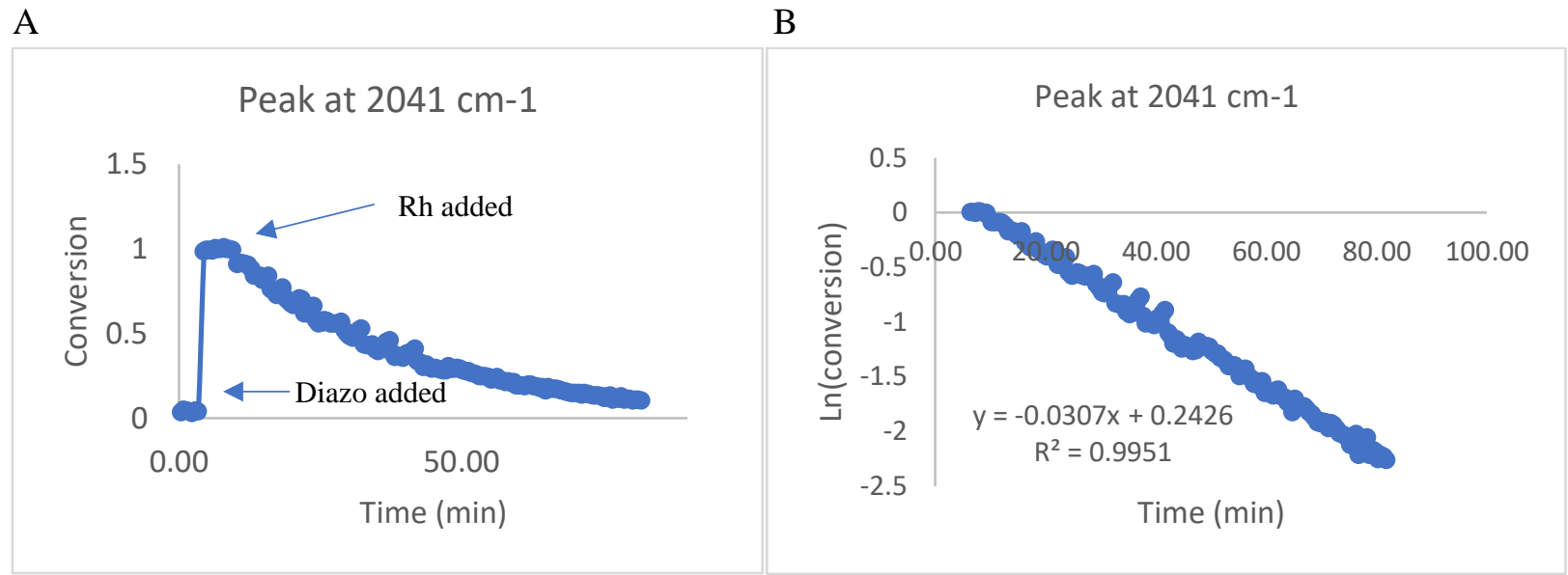

Figure S11. A: Data graphed for reaction with diazo compound 3a catalyzed by $\mathrm{Rh}_{2}(\mathrm{~S}$-TCPTTL)4: A) Graph of conversion vs. time. B) Graph of $\ln [$ conv] vs. time once catalyst was added.

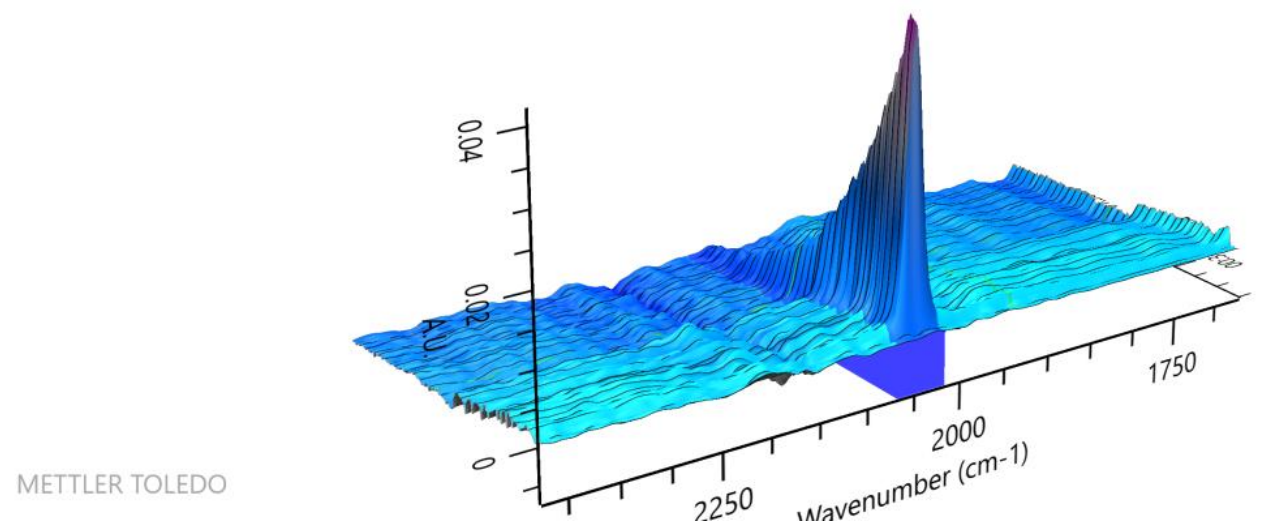

Figure S12. ReactIR surface plot for trial 1 
Trial 2

A B
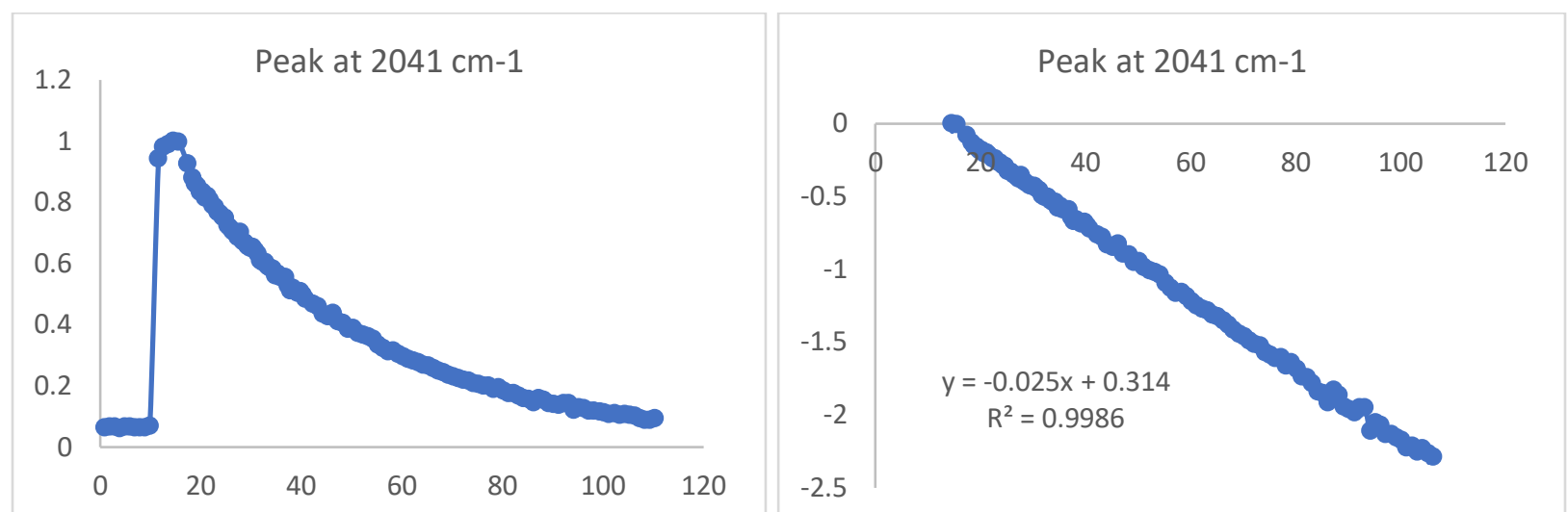

Figure S13. A: Data graphed for reaction with diazo compound 3a catalyzed by $\mathrm{Rh}_{2}(S$-TCPTTL)4: A) Graph of conversion vs. time. B) Graph of $\ln [$ conv] vs. time once catalyst was added.

Based on the data observed, $k_{\text {rel }}=k_{2} \mathrm{a} / k_{3 \mathrm{a}}=117.8$ for trial 1 and 127.2 for trial 2. 


\section{Procedure for kinetic isotope effect (Figure 2)}

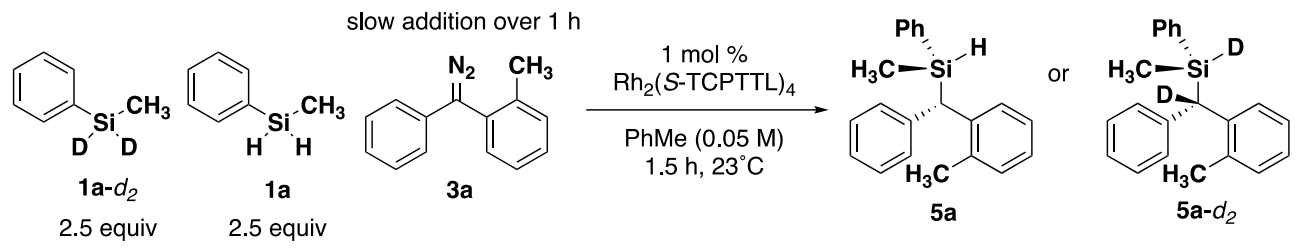

A $8 \mathrm{~mL}$ reaction vial equipped with stir bar and $100 \mathrm{mg} 4 \AA$ molecular sieves was heated under flame and dried under high vacuum ( $>1$ torr). After the vial cooled to room temperature, it was purged with argon, followed by addition of $\mathrm{Rh}_{2}(S \text {-TCPTTL })_{4}(0.002 \mathrm{mmol}, 3.6 \mathrm{mg})$ and silane $1 \mathrm{a}(1 \mathrm{mmol}, 61 \mathrm{mg})$ and silane $1 \mathbf{a}-d_{2}(0.5 \mathrm{mmol}, 61 \mathrm{mg})$. The vial was re-purged with argon, and $2 \mathrm{~mL}$ of PhMe was added. Diazo compound $3 \mathbf{a}(0.2 \mathrm{mmol}, 39.0 \mathrm{mg}))$ was weighed into a separate flame-dried vial, and $2 \mathrm{~mL}$ of PhMe was added, then drawn into a syringe. Using a long needle, the syringe was placed on a syringe pump with the needle in the stirring solution. The syringe pump was programed to add the solution over a period of $1 \mathrm{~h}$ at room temperature.

Thirty minutes after diazo addition, the solution was diluted with $5 \mathrm{~mL}$ of hexane, and filtered through celite, and concentrated in vacuo. The crude mixture was diluted in hexanes and ran through a short silica plug $(4 \mathrm{~cm}$ in a Pasteur pipette) to remove catalyst, and concentrated in vасио. The product was then purified using column chromatography (dry loaded sample in silica, 98:2 hexanes/DCM) to furnish a mixture of silane $\mathbf{5 a}$ and $\mathbf{5 a}-d_{2}$ as an oil.

The kinetic isotope effect was determined using an integration ratio of the benzhydryl resonance for the major diastereomer present at 3.78. ppm and both benzyl resonances observed at $2.25 \mathrm{ppm}$ (major diastereomer) using Equation 1:

$$
K I E=\frac{I_{A}}{\frac{I_{B}}{3}-I_{A}}
$$

Where $I_{A}$ is the integral of the benzhydryl resonance, and $I_{B}$ is the integral of both benzyl resonances. This equation takes into account both stoichiometry and the combined integration of both $\mathbf{5 a}$ and $\mathbf{5 a}-d_{2}$ at $2.25 \mathrm{ppm}$.

Trial 1

$I_{A}=1.00$

$I_{B}=4.91$

$\mathrm{KIE}=1.57$

Trial 2

$I_{A}=1.0$

$I_{B}=4.90$

$\mathrm{KIE}=1.58$

Based on the trials 1 and 2 , the calculated KIE is 1.575, which is rounded to 1.6. 
${ }^{1} \mathrm{H} \mathrm{NMR}, \mathrm{CDCl}_{3}, 600 \mathrm{MHz}$

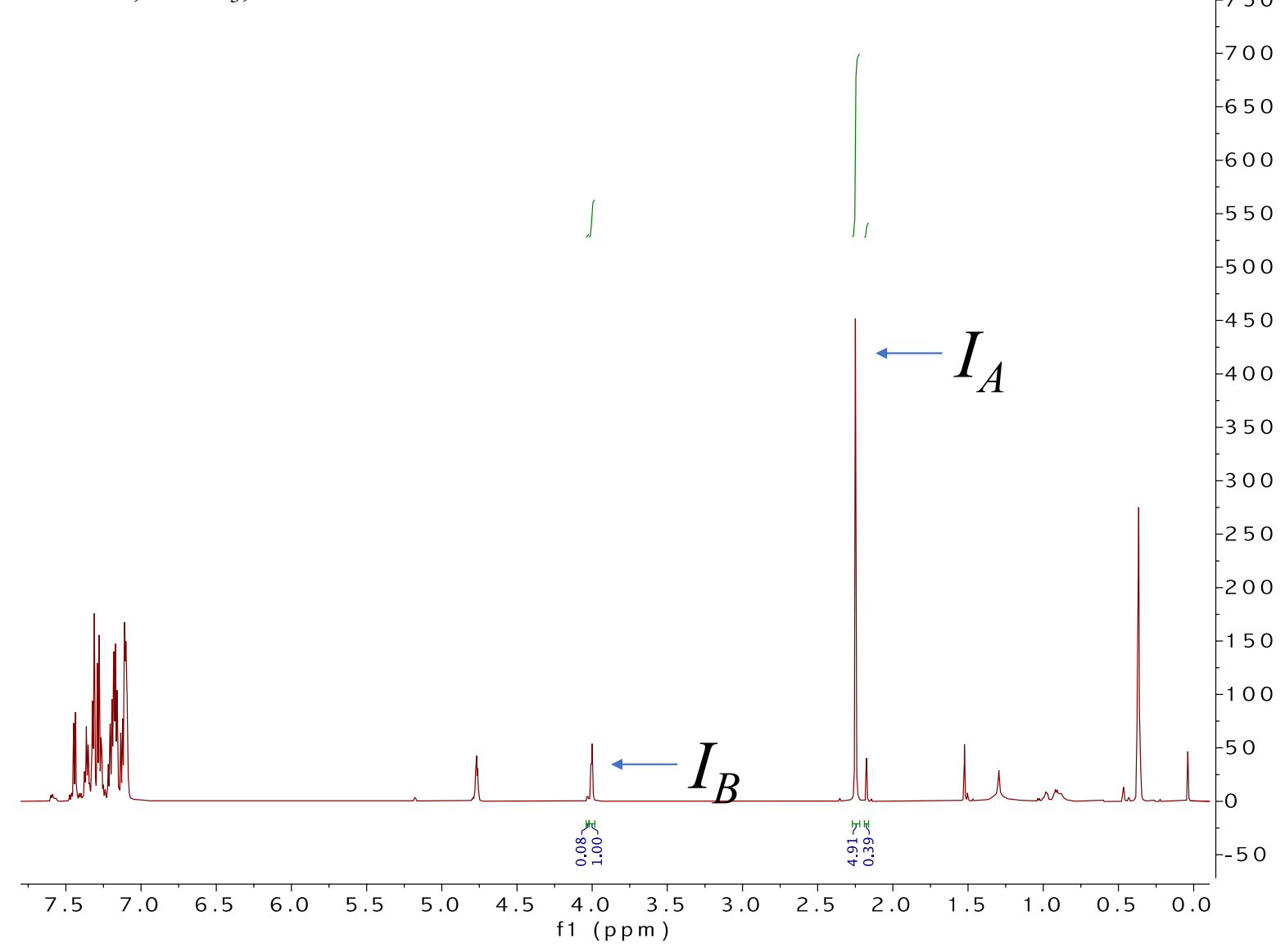

Figure S14. $1 \mathrm{H}$ NMR of 5a and 5a- $d_{2}$ mixture, with $I_{A}$ and $I_{B}$ shown. 


\section{Characterization Data}

\section{Silanes:}

\section{methyl(phenyl)silane- $d_{2}\left(1 \mathrm{a}-d_{2}\right)$}

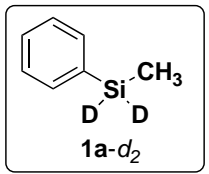

Made using previously reported procedures. 13 Spectrrum matches with previous report. 13

1H NMR (600 MHz, CDCl $) \delta 7.59(\mathrm{~m}, 2 \mathrm{H}), 7.45-7.35(\mathrm{~m}, 3 \mathrm{H}), 0.43(\mathrm{~s}, 3 \mathrm{H})$.

\section{(4-fluorophenyl)(methyl)silane (1b)}

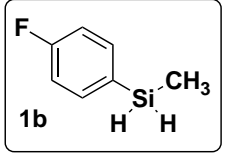

Synthesized according to the general procedure B for silane formation using 1-bromo-4fluorobenzene $(1.88 \mathrm{~mL}, 15.0 \mathrm{mmol}), n$-BuLi $(15.0 \mathrm{mmol}, 6 \mathrm{~mL}$ solution in hexanes) and dichloromethylsilane $(1.09 \mathrm{~mL}, 15.0 \mathrm{mmol})$ and purified using bulb-to-bulb distillation to give a clear liquid that can be isolated in $45 \%$ yield $(1.15 \mathrm{~g}, 6.75 \mathrm{mmol})$ over 2 steps from addition of the chlorosilane.

1H NMR (600 MHz, $\left.\mathrm{C}_{6} \mathrm{D}_{6}\right) \delta 7.19-7.16(\mathrm{~m}, 1 \mathrm{H}), 6.79(\mathrm{t}, J=8.7 \mathrm{~Hz}, 1 \mathrm{H}), 4.37(\mathrm{q}, J=4.3 \mathrm{~Hz}, 1 \mathrm{H}), 0.10(\mathrm{t}, J=4.3$ $\mathrm{Hz}, 1 \mathrm{H}) .13 \mathrm{C}$ NMR $\left(101 \mathrm{MHz}, \mathrm{CDCl}_{3}\right) \delta 164.2(\mathrm{~d}, J=248.7 \mathrm{~Hz}), 137.0(\mathrm{~d}, J=7.6 \mathrm{~Hz}), 128.9(\mathrm{~d}, J=3.8 \mathrm{~Hz}), 115.4$ $(\mathrm{d}, J=19.9 \mathrm{~Hz}),-7.3 .{ }_{19} \mathrm{~F}$ NMR (376 MHz, Chloroform- $d$ ) $\delta$-111.16. $29 \mathrm{Si}$ NMR (119 MHz, $\left.\mathrm{CDCl}_{3}\right) \delta-35.8$

\section{(4-methoxyphenyl)(methyl)silane (1c)}

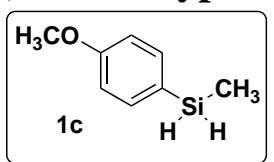

Synthesized according to the general procedure for silane formation using 4-bromoanisole (1.88 $\mathrm{mL}, 15.0 \mathrm{mmol})$ and dichloromethylsilane $(1.09 \mathrm{~mL}, 15.0 \mathrm{mmol})$ and purified using column chromatography (hexanes) to give a clear liquid that can be isolated in $81 \%$ yield $(1.85 \mathrm{~g}, 12.2$ $\mathrm{mmol}$ ) over 2 steps from addition of the chlorosilane.

1H NMR (600 MHz, C6D6) $\delta .41(\mathrm{~d}, J=8.1 \mathrm{~Hz}, 2 \mathrm{H}), 6.80(\mathrm{~d}, J=8.0 \mathrm{~Hz}, 2 \mathrm{H}), 4.55$ (q, $J=4.0 \mathrm{~Hz}, 2 \mathrm{H}), 3.27(\mathrm{~s}, 3 \mathrm{H})$, $0.23(\mathrm{t}, J=4.1 \mathrm{~Hz}, 3 \mathrm{H}) .{ }_{13} \mathrm{C}$ NMR $\left(100 \mathrm{MHz}, \mathrm{CDCl}_{3}\right) \delta 161.0,136.5,124.2,114.0,55.2,-7.2 .29 \mathrm{Si} \mathrm{NMR}(119 \mathrm{MHz}$, $\left.\mathrm{CDCl}_{3}\right) \delta-36.4$.

\section{$\operatorname{Methyl}(p$-tolyl)silane (1d)}

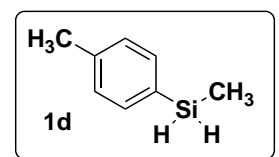

Synthesized according to the general procedure A for silane formation using 1-bromo-4methylbenzene $(1.85 \mathrm{~mL}, 15.0 \mathrm{mmol})$ and dichloromethylsilane $(1.09 \mathrm{~mL}, 15.0 \mathrm{mmol})$ and purified using bulb-to-bulb distillation to give a clear liquid that can be isolated in $63 \%$ yield $(1.29 \mathrm{~g})$ over 2 steps from the addition of the chlorosilane.

1H NMR (600 MHz, C6 6 6) $\delta 7.42(\mathrm{~d}, J=7.5 \mathrm{~Hz}, 2 \mathrm{H}), 7.01(\mathrm{~d}, J=7.5 \mathrm{~Hz}, 2 \mathrm{H}), 4.53(\mathrm{q}, J=4.1 \mathrm{~Hz}, 2 \mathrm{H}), 2.09(\mathrm{~s}, 3 \mathrm{H})$, $0.22(\mathrm{t}, J=4.1 \mathrm{~Hz}, 3 \mathrm{H}) .13 \mathrm{C} \mathrm{NMR}\left(101 \mathrm{MHz}, \mathrm{CDCl}_{3}\right) \delta 139.6,135.0,129.8,129.0,21.6,-7.4 .29 \mathrm{Si} \mathrm{NMR}(119 \mathrm{MHz}$, $\left.\mathrm{CDCl}_{3}\right) \delta-36.1$. 


\section{Methyl(naphthalen-2-yl)silane (1e)}

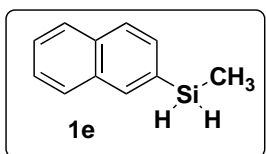

Synthesized according to the general procedure A for silane formation using 2bromonaphthalene $(3.26 \mathrm{~g})$ in $5 \mathrm{~mL}$ THF and dichloromethylsilane $(1.09 \mathrm{~mL}, 15.0 \mathrm{mmol})$ and purified using bulb-to-bulb distillation via kugelrohr $\left(150^{\circ} \mathrm{C}, 20\right.$ torr) followed by sublimation $\left(70^{\circ} \mathrm{C}, 760\right.$ torr) to give a clear liquid that can be isolated in $50 \%$ yield $(1.29 \mathrm{~g}, 7.5 \mathrm{mmol}$, based on $\mathrm{Si}$ ) over 2 steps from the addition of chlorosilane.

${ }_{1} \mathrm{H}$ NMR $\left(600 \mathrm{MHz}, \mathrm{C}_{6} \mathrm{D} 6\right) \delta 7.99(\mathrm{~s}, 1 \mathrm{H}), 7.61(\mathrm{t}, J=7.2 \mathrm{~Hz}, 3 \mathrm{H}), 7.49(\mathrm{~d}, J=8.1 \mathrm{~Hz}, 2 \mathrm{H}), 7.30-7.22(\mathrm{~m}, 2 \mathrm{H}), 4.60$ $(\mathrm{q}, J=4.3 \mathrm{~Hz}, 0 \mathrm{H}), 0.25(\mathrm{t}, J=4.2 \mathrm{~Hz}, 1 \mathrm{H}) .13 \mathrm{C} \mathrm{NMR}\left(101 \mathrm{MHz}, \mathrm{CDCl}_{3}\right) \delta 136.0,134.0,133.1,131.0,130.9,128.1$, 127.9, 127.4, 126.7, 126.2, -7.4. 29Si NMR (119 MHz, $\left.\mathrm{CDCl}_{3}\right) \delta$-35.4.

\section{Methyl(naphthalen-1-yl)silane (1f)}

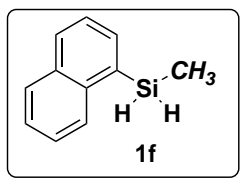

Synthesized according to the general procedure $\mathrm{B}$ for silane formation using 1-bromonaphthalene $(2.10 \mathrm{~mL}, 15.0 \mathrm{mmol}), n$-BuLi $(15.0 \mathrm{mmol}, 6 \mathrm{~mL}$ solution in hexanes) and dichloromethylsilane $(1.09 \mathrm{~mL}, 15.0 \mathrm{mmol})$ and purified using bulb-to-bulb distillation via kugelrohr $\left(150^{\circ} \mathrm{C}, 20\right.$ torr $)$ followed by sublimation $\left(70^{\circ} \mathrm{C}, 760\right.$ torr) to give a clear liquid that can be isolated in $50 \%$ yield $(1.29 \mathrm{~g}, 7.5 \mathrm{mmol})$ over 2 steps from addition of the chlorosilane.

${ }_{1} \mathrm{H}$ NMR $\left(600 \mathrm{MHz}, \mathrm{CDCl}_{3}\right) \delta 8.04(\mathrm{~d}, J=8.2 \mathrm{~Hz}, 1 \mathrm{H}), 7.64-7.54(\mathrm{~m}, 3 \mathrm{H}), 7.29-7.23(\mathrm{~m}, 1 \mathrm{H}), 7.24-7.19(\mathrm{~m}$, $1 \mathrm{H}), 7.19-7.14(\mathrm{~m}, 1 \mathrm{H}), 4.75(\mathrm{q}, J=4.2 \mathrm{~Hz}, 2 \mathrm{H}), 0.24(\mathrm{t}, J=4.2 \mathrm{~Hz}, 3 \mathrm{H}) .{ }_{13} \mathrm{C} \mathrm{NMR}\left(151 \mathrm{MHz}, \mathrm{CDCl}_{3}\right) \delta 137.2$, 135.3, 133.2, 132.2, 130.6, 129.0, 127.7, 126.3, 125.9, 125.4, -7.2. 29Si NMR (119 MHz, CDCl3) $\delta-38.6$.

\section{(4-fluoronaphthalen-1-yl)(methyl)silane (1g)}

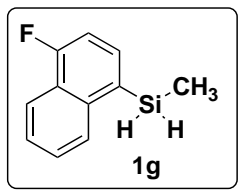

Synthesized according to the general procedure for silane formation using 4-fluoro-1bromonaphthalene (3.38 g, $15.0 \mathrm{mmol}$ in $5 \mathrm{~mL}$ THF) and dichloromethylsilane $(1.09 \mathrm{~mL}, 15.0$ mmol) $)$ and purified using bulb-to-bulb distillation via kugelrohr $\left(150{ }^{\circ} \mathrm{C}, 50\right.$ torr for $30 \mathrm{~min}$ then $105^{\circ} \mathrm{C}, 10$ torr to give a clear liquid that can be isolated in $21 \%$ yield $(597.0 \mathrm{mg}, 3.1 \mathrm{mmol}$, based off $\mathrm{Si}$ ) over 2 steps from addition of the chlorosilane.

1H NMR (600 MHz, CDCl $) \delta 8.18(\mathrm{~d}, J=8.0 \mathrm{~Hz}, 1 \mathrm{H}), 8.05(\mathrm{~d}, J=8.0 \mathrm{~Hz}, 1 \mathrm{H}), 7.72(\mathrm{t}, J=6.7 \mathrm{~Hz}, 1 \mathrm{H}), 7.60(\mathrm{dt}, J$ $=16.1,7.0 \mathrm{~Hz}, 2 \mathrm{H}), 7.18-7.12(\mathrm{~m}, 1 \mathrm{H}), 4.65(\mathrm{q}, J=4.2,3.7 \mathrm{~Hz}, 2 \mathrm{H}), 0.54(\mathrm{t}, J=3.9 \mathrm{~Hz}, 3 \mathrm{H}) .13 \mathrm{C} \mathrm{NMR}(150 \mathrm{MHz}$, $\mathrm{CDCl}_{3} \delta 160.78\left(\mathrm{~d}, J_{C F}=255.0 \mathrm{~Hz}\right), 138.82\left(\mathrm{~d}, J_{C C C F}=4.4 \mathrm{~Hz}\right), 135.28\left(\mathrm{~d}, J_{\mathrm{CCF}}=8.3 \mathrm{~Hz}\right), 127.80\left(\mathrm{~d}, J_{C C C F}=4.8 \mathrm{~Hz}\right)$, $127.57(\mathrm{~d}, J=3.1 \mathrm{~Hz}), 127.32,126.21\left(\mathrm{~d}, J_{C C C C F}=1.7 \mathrm{~Hz}\right), 121.40\left(\mathrm{~d}, J_{C C C F}=6.1 \mathrm{~Hz}\right), 109.17\left(\mathrm{~d}, J_{C C F}=18.6 \mathrm{~Hz}\right),-$ 7.19. ${ }_{19} \mathrm{~F}$ NMR (376 MHz, $\left.\mathrm{CDCl}_{3}\right) \delta$-120.24. $29 \mathrm{Si} \mathrm{NMR}\left(119 \mathrm{MHz}, \mathrm{CDCl}_{3}\right) \delta-35.8$

\section{$\operatorname{Methyl(o-tolyl)silane~(1h)~}$}

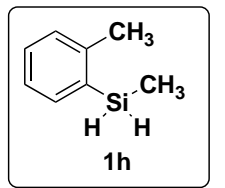

Synthesized according to the general procedure for silane formation using 2-bromotoluene (1.80 $\mathrm{mL}, 15.0 \mathrm{mmol})$ and dichloromethylsilane $(1.09 \mathrm{~mL}, 15.0 \mathrm{mmol})$ and purified using column chromatography (hexanes) to give a clear liquid that can be isolated in $72 \%$ yield $(1.47 \mathrm{~g}, 10.8 \mathrm{mmol})$ over 2 steps from the addition of the chlorosilane. Matches with previously reported spectra. 14

1H NMR (600 MHz, $\left.\mathrm{CDCl}_{3}\right) \delta 7.52(\mathrm{~d}, J=7.3 \mathrm{~Hz}, 1 \mathrm{H}), 7.32(\mathrm{t}, J=7.5 \mathrm{~Hz}, 1 \mathrm{H}), 7.21-7.16(\mathrm{~m}, 2 \mathrm{H}), 4.37(\mathrm{q}, J=4.2$ $\mathrm{Hz}, 2 \mathrm{H}), 2.46$ (s, 3H), 0.43 (t, $J=4.2 \mathrm{~Hz}, 3 \mathrm{H})$. 


\section{Mesityl(methyl)silane (1i)}

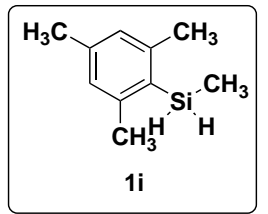

Synthesized according to the general procedure for silane formation using 2-bromomesitylene $(2.30 \mathrm{~mL}, 15 \mathrm{mmol})$ and dichloromethylsilane $(1.09 \mathrm{~mL}, 15.0 \mathrm{mmol})$ and purified using column chromatography (hexanes) to give a clear liquid that can be isolated in $87 \%$ yield $(2.14 \mathrm{~g}, 13.0$ $\mathrm{mmol}$ ) over 2 steps from addition of the chlorosilane. Spectral data matches previously reported values. 14

1H NMR (400 MHz, CDCl 3$) \delta 6.88(\mathrm{~s}, 2 \mathrm{H}), 4.45(\mathrm{q}, J=4.2 \mathrm{~Hz}, 2 \mathrm{H}), 2.47(\mathrm{~s}, 6 \mathrm{H}), 2.30(\mathrm{~s}, 3 \mathrm{H}), 0.36(\mathrm{t}, J=4.2 \mathrm{~Hz}$, $3 \mathrm{H})$.

\section{Isobutyl(naphthalen-1-yl)silane (1j)}

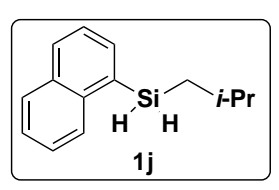

Synthesized according to Method $\mathrm{C}$ for silane formation using 1-bromonaphthalene $(2.10 \mathrm{~mL}$, $15.0 \mathrm{mmol}), n-\mathrm{BuLi}$ (15.0 mmol, $6 \mathrm{~mL}$ solution in hexanes), and dichlorosiobutylsilane (2.28 $\mathrm{mL}, 15.0 \mathrm{mmol})$ and purified using bulb-to-bulb distillation via kugelrohr $\left(150{ }^{\circ} \mathrm{C}, 20\right.$ torr $)$ followed by sublimation $\left(70^{\circ} \mathrm{C}, 760\right.$ torr) that can be isolated as a clear liquid in $21 \%$ yield (675.0 mg, $3.2 \mathrm{mmol}$, based of $\mathrm{Si}$ ) over 2 steps from addition of the chlorosilane.

1H NMR (600 MHz, C6 $\left.\mathrm{D}_{6}\right) \delta 8.14(\mathrm{~m}, 1 \mathrm{H}), 7.74(\mathrm{~m}, 1 \mathrm{H}), 7.65(\mathrm{~m}, 2 \mathrm{H}), 7.33(\mathrm{~m}, 1 \mathrm{H}), 7.27(\mathrm{~m}, 1 \mathrm{H}), 7.23(\mathrm{~m}, 1 \mathrm{H})$, $4.83(\mathrm{t}, J=3.8 \mathrm{~Hz}, 2 \mathrm{H}), 1.76(\mathrm{hept}, J=6.7 \mathrm{~Hz}, 1 \mathrm{H}), 0.94(\mathrm{dd}, J=7.1,3.7 \mathrm{~Hz}, 2 \mathrm{H}), 0.91(\mathrm{~d}, J=6.6 \mathrm{~Hz}, 6 \mathrm{H}) .13 \mathrm{C} \mathrm{NMR}$ $\left(151 \mathrm{MHz}, \mathrm{CDCl}_{3}\right) \delta 137.37,135.85,133.23,131.87,130.53,129.04,127.92,126.31,125.86,125.39,26.09,25.62$, 20.80. 29Si NMR (79 MHz, $\left.\mathrm{CDCl}_{3}\right) \delta$-36.0.

\section{Isobutyl(phenyl)silane (1k)}

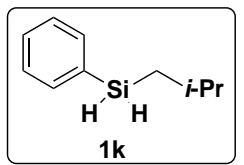

Synthesized according to method $\mathrm{C}$ for silane formation using bromobenzene $(1.58 \mathrm{~mL}, 15.0$ mmol) and dichlorosiobutylsilane $(2.28 \mathrm{~mL}, 15.0 \mathrm{mmol})$ and purified using bulb-to-bulb distillation $\left(150{ }^{\circ} \mathrm{C}, 100\right.$ torr) to give a liquid that can be isolated as a clear liquid in $41 \%$ yield $(1.01 \mathrm{~g}, 6.15 \mathrm{mmol})$ over 2 steps from addition of the chlorosilane.

1H NMR (600 MHz, $\left.\mathrm{CDCl}_{3}\right) \delta 7.57(\mathrm{~m}, 2 \mathrm{H}), 7.36(\mathrm{~m}, 3 \mathrm{H}), 4.31(\mathrm{t}, J=3.9 \mathrm{~Hz}, 2 \mathrm{H}), 1.85(\mathrm{dh}, J=13.4,6.7 \mathrm{~Hz}, 1 \mathrm{H})$, $0.99(\mathrm{~d}, J=6.6 \mathrm{~Hz}, 6 \mathrm{H}), 0.95(\mathrm{dt}, J=7.5,3.9 \mathrm{~Hz}, 2 \mathrm{H}) .13 \mathrm{C} \mathrm{NMR}\left(151 \mathrm{MHz}, \mathrm{CDCl}_{3}\right) \delta 135.35,133.10,129.58,128.09$, 25.75, 25.62, 20.61. 29Si NMR (119 MHz, $\left.\mathrm{CDCl}_{3}\right) \delta-33.8$.

\section{Neopentyl(phenyl)silane (11)}

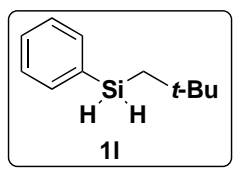

Synethsized using method $\mathrm{H}$. Isolated as a clear liquid in $25 \%$ yield (231 mg, $1.3 \mathrm{mmol})$.

1H NMR (600 MHz, CDCl $) \delta 7.57(\mathrm{~d}, J=6.4 \mathrm{~Hz}, 1 \mathrm{H}), 7.36$ (hept, $J=6.7,6.2 \mathrm{~Hz}, 2 \mathrm{H}), 4.34(\mathrm{t}, J=4.2 \mathrm{~Hz}, 2 \mathrm{H}), 1.08$ $(\mathrm{t}, J=4.2 \mathrm{~Hz}, 2 \mathrm{H}), 1.04(\mathrm{~s}, 9 \mathrm{H}) .13 \mathrm{C}$ NMR $\left(151 \mathrm{MHz}, \mathrm{CDCl}_{3}\right) \delta 135.35,133.53,129.51,128.08,33.07,32.31,27.09$. ${ }_{29} \mathrm{Si} \mathrm{NMR}\left(79 \mathrm{MHz}, \mathrm{CDCl}_{3}\right) \delta-37.8$.

\section{Cyclohexylphenylsilane (1m)}

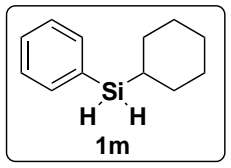

Made using previously reported procedures.15 Spectra matches with previous report.

1H NMR (600 MHz, $\left.\mathrm{CDCl}_{3}\right) \delta 7.55(\mathrm{dt}, J=6.5,1.5 \mathrm{~Hz}, 2 \mathrm{H}), 7.41-7.37(\mathrm{~m}, 1 \mathrm{H}), 7.37-7.33(\mathrm{~m}, 2 \mathrm{H}), 4.16(\mathrm{~d}, J=$ $3.0 \mathrm{~Hz}, 2 \mathrm{H}), 1.83-1.58(\mathrm{~m}, 7 \mathrm{H}), 1.22-0.91(\mathrm{~m}, 4 \mathrm{H})$. 


\section{1,1,1-Triethyl-3-phenyldisiloxane (1n)}

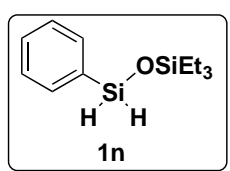

Made using previously reported procedure.3 Spectra matches with previous report.

1H NMR (400 MHz, $\left.\mathrm{CDCl}_{3}\right) \delta 7.69-7.60(\mathrm{~m}, 2 \mathrm{H}), 7.52-7.35(\mathrm{~m}, 3 \mathrm{H}), 5.14(\mathrm{~s}, 1 \mathrm{H}), 0.96(\mathrm{t}, J=8.0 \mathrm{~Hz}, 9 \mathrm{H}), 0.60$ $(\mathrm{q}, J=8.0 \mathrm{~Hz}, 6 \mathrm{H})$.

\section{Diazo compounds:}

\section{Diphenyldiazomethane (2a)}

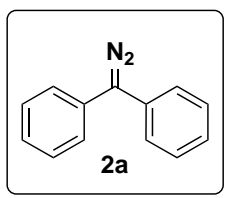

Synthesized using previously reported procedures. Spectrum matches previous report.7

1H NMR (600 MHz, CDCl 3$) \delta 7.39(\mathrm{t}, J=7.7 \mathrm{~Hz}, 3 \mathrm{H}), 7.30(\mathrm{~d}, J=8.4 \mathrm{~Hz}, 2 \mathrm{H}), 7.19(\mathrm{t}, J=7.4 \mathrm{~Hz}, 1 \mathrm{H})$.

\section{4,4'-(diazomethylene)bis(methoxybenzene) (2b)}

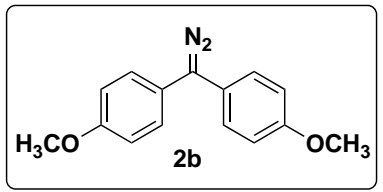

Made using previously published procedures. Spectrum matches previous report.7

1H NMR (600 MHz, CDCl3) $\delta 7.18(\mathrm{~d}, J=8.8 \mathrm{~Hz}, 4 \mathrm{H}), 6.94(\mathrm{~d}, J=8.8 \mathrm{~Hz}, 4 \mathrm{H}), 3.82(\mathrm{~s}, 3 \mathrm{H})$.

\section{4,4'-(diazomethylene)bis(chlorobenzene) (2c)}

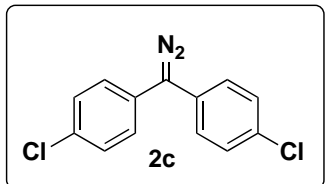

Made using previously published procedures. Spectrum matches previous report.7

1H NMR (400 MHz, CDCl 3$) \delta 7.36(\mathrm{~d}, J=8.8 \mathrm{~Hz}, 4 \mathrm{H}), 7.18(\mathrm{~d}, J=8.7 \mathrm{~Hz}, 4 \mathrm{H})$.

\section{Phenyl(o-tolyl)diazomethane (3a)}

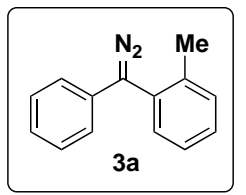

Synthesized using procedure A for diazo formation using 2-methylbenzophenone $(0.36 \mathrm{~mL}, 2.0$ $\mathrm{mmol})$ to give a purple liquid that can be isolated in $60 \%$ yield $(243.0 \mathrm{mg})$ over 2 steps.

${ }_{1} \mathrm{H} \mathrm{NMR}\left(400 \mathrm{MHz}, \mathrm{CDCl}_{3} \delta 7.43(\mathrm{~d}, J=7.1 \mathrm{~Hz}, 1 \mathrm{H}), 7.31(\mathrm{~m}, 5 \mathrm{H}), 7.07(\mathrm{t}, J=7.3 \mathrm{~Hz}, 2 \mathrm{H}), 6.97(\mathrm{~d}, J=7.9 \mathrm{~Hz}, 1 \mathrm{H})\right.$, 2.28 (s, 3H). ${ }_{13} \mathrm{C}$ NMR $\left(151 \mathrm{MHz}, \mathrm{CDCl}_{3}\right) \delta 138.5,131.5,131.25,131.0,129.1,128.7,127.2,126.7,124.1,122.6$, 20.6. APCI $\mathrm{m} / \mathrm{z}$ calc for $\mathrm{C}_{14} \mathrm{H}_{12} \mathrm{~N}_{2}\left[\mathrm{M}+\mathrm{H}-\mathrm{N}_{2}\right]$ 


\section{Phenyl(o-methoxyphenyl)diazomethane (3b)}

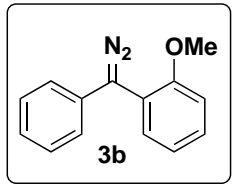

Synthesized using procedure A using 2-methoxybenzphenone $(457.0 \mathrm{mg}, 2.2 \mathrm{mmol})$ to give a purple oil that can be isolated in $68 \%$ yield $(761.9 \mathrm{mg})$ over 2 steps.

1H NMR (600 MHz, $\left.\mathrm{CDCl}_{3}\right) \delta 7.37(\mathrm{~d}, J=7.6 \mathrm{~Hz}, 1 \mathrm{H}), 7.31(\mathrm{q}, J=6.9 \mathrm{~Hz}, 3 \mathrm{H}), 7.08(\mathrm{~m}, 3 \mathrm{H}), 7.04-6.96(\mathrm{~m}, 2 \mathrm{H})$, $3.86(\mathrm{~s}, 3 \mathrm{H}) .13 \mathrm{C}$ NMR $\left(101 \mathrm{MHz}, \mathrm{CDCl}_{3}\right) \delta 157.0,131.6,130.1,129.0,124.2,123.2,121.2,117.2,111.7,55.7$. APCI $m / z$ calc for $\mathrm{C}_{14} \mathrm{H}_{12} \mathrm{~N}_{2} \mathrm{O}\left[\mathrm{M}+\mathrm{H}-\mathrm{N}_{2}\right]_{+}$. 197.1. Found 196.5.

\section{Phenyl(o-trifluoromethylphenyl)diazomethane (3c)}

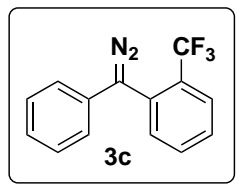

Synthesized using method B to give a red liquid that can be isolated in $44 \%$ yield $(461.5 \mathrm{mg}$ ) over 2 steps.

1H NMR (400 MHz, CDCl $) \delta 7.83(\mathrm{~d}, J=7.9 \mathrm{~Hz}, 1 \mathrm{H}), 7.59(\mathrm{ddt}, J=23.5,15.2,7.6 \mathrm{~Hz}, 3 \mathrm{H}), 7.31(\mathrm{t}, J=7.7 \mathrm{~Hz}, 2 \mathrm{H})$, $7.08(\mathrm{t}, J=7.4 \mathrm{~Hz}, 1 \mathrm{H}), 6.86(\mathrm{~d}, J=7.9 \mathrm{~Hz}, 2 \mathrm{H}) .13 \mathrm{C} \mathrm{NMR}\left(101 \mathrm{MHz}, \mathrm{CDCl}_{3}\right) \delta 134.3,132.6,131.1\left(\mathrm{q}, J_{C C F}=30\right.$ $\mathrm{Hz}), 131.6,129.2,129.1127 .4\left(\mathrm{q}, J_{C C C F}=5.4 \mathrm{~Hz}\right), 127.0,124.3,124.0\left(\mathrm{q}, J_{C F}=275 \mathrm{~Hz}\right) 122.5 .19 \mathrm{~F} \mathrm{NMR}(376 \mathrm{MHz}$, $\left.\mathrm{CDCl}_{3}\right) \delta$-61.93. APCI $m / z$ calc for $\mathrm{C}_{14} \mathrm{H}_{9} \mathrm{~F}_{3} \mathrm{~N}_{2}\left[\mathrm{M}+\mathrm{H}-\mathrm{N}_{2}\right]_{+}$. 235.1. Found 234.4.

\section{1-chloro-2-(diazo(4-methoxyphenyl)methyl)benzene (3d)}

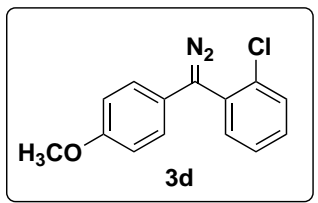

Synthesized according to previous procedures and isolated as a red oil.16 Spectrum matches previous report. 16

1H NMR (600 MHz, CDCl $) \delta 7.48(\mathrm{~d}, J=7.8 \mathrm{~Hz}, 1 \mathrm{H}), 7.42(\mathrm{~d}, J=5.9 \mathrm{~Hz}, 1 \mathrm{H}), 7.30(\mathrm{~d}, J=7.5 \mathrm{~Hz}, 1 \mathrm{H}), 7.27(\mathrm{~m}, J$ $2 \mathrm{H}), 6.96(\mathrm{~d}, J=7.0 \mathrm{~Hz}, 2 \mathrm{H}), 6.91(\mathrm{~d}, J=9.0 \mathrm{~Hz}, 3 \mathrm{H}), 3.80(\mathrm{~s}, 1 \mathrm{H})$.

\section{Phenyl(1-naphthyl)diazomethane (3e)}

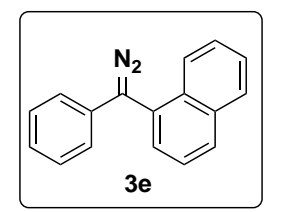

Synthesized according to the general procedure for diazo formation using 1-napthylphenylketone $(1.340 \mathrm{~g}, 5.7 \mathrm{mmol})$ to give a purple solid that can be isolated in $68 \%$ yield $(828.9 \mathrm{mg})$ over 2 steps.

1H NMR (600 MHz, $\left.\mathrm{CDCl}_{3}\right) \delta 7.97-7.90(\mathrm{~m}, 2 \mathrm{H}), 7.88(\mathrm{~d}, J=8.2 \mathrm{~Hz}, 1 \mathrm{H}), 7.69(\mathrm{~d}, J=6.7 \mathrm{~Hz}, 1 \mathrm{H}), 7.56(\mathrm{dt}, J=$ 21.5, 7.3 Hz, 2H), $7.49(\mathrm{t}, J=7.4 \mathrm{~Hz}, 1 \mathrm{H}), 7.31(\mathrm{t}, J=7.1 \mathrm{~Hz}, 2 \mathrm{H}), 7.08(\mathrm{t}, J=7.1 \mathrm{~Hz}, 1 \mathrm{H}), 6.97(\mathrm{~d}, J=7.5 \mathrm{~Hz}, 2 \mathrm{H})$. 13C NMR (151 MHz, $\left.\mathrm{CDCl}_{3}\right) \delta 134.5,132.2,131.9,129.5,129.3,129.1,128.9,126.8,126.5,126.0,125.6,125.44$, 124.2, 122.7. APCI $m / z$ calc for $\mathrm{C}_{17} \mathrm{H}_{12} \mathrm{~N}_{2}\left[\mathrm{M}+\mathrm{H}-\mathrm{N}_{2}\right]_{+}$. 217.1. Found 216.5. 


\section{Insertion Products:}

\section{(S)-benzhydryl(methyl)(phenyl)silane (4a)}

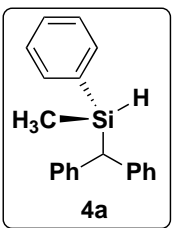

Synthesized using method F with diazo compound $2 \mathbf{2 a}(1.0 \mathrm{mmol}, 181.0 \mathrm{mg})$ in $\mathrm{PhMe}(20 \mathrm{~mL}$ total) to give a white solid in $78 \%$ yield $(0.78 \mathrm{mmol}, 225.0 \mathrm{mg})$. Enantiomeric ratio was determined by HLPC after hydrolysis with a Daicel CHIRALPAK ® OD-H column (2\% IPA/ hexanes), $1.0 \mathrm{~mL} / \mathrm{min}$. $\mathrm{tR}_{\mathrm{R}}(\mathbf{4 a a})=11.3 \mathrm{~min}$, $\mathrm{tR}(\mathbf{4 a b})=12.9 \mathrm{~min}, 82: 18 \mathrm{er}(\mathrm{Si}-\mathrm{OH}$ product). Absolute configuration was assigned to be $(S)$ based on analogy to 5 a.

${ }_{1} \mathrm{H}$ NMR $\left(400 \mathrm{MHz}_{\mathrm{CDCl}}\right) \delta 7.32(\mathrm{~m}, 2 \mathrm{H}), 7.28-7.21(\mathrm{~m}, 7 \mathrm{H}), 7.18(\mathrm{~m}, 4 \mathrm{H}), 7.12(\mathrm{~m}, 2 \mathrm{H}), 4.84-4.59(\mathrm{~m}, 1 \mathrm{H})$, $3.81(\mathrm{~d}, J=3.7 \mathrm{~Hz}, 1 \mathrm{H}), 0.30(\mathrm{~d}, J=3.5 \mathrm{~Hz}, 3 \mathrm{H}) .{ }_{13} \mathrm{C} \mathrm{NMR}\left(101 \mathrm{MHz}, \mathrm{CDCl}_{3}\right) \delta 142.3,142.1,135.0,134.8,129.6$, $129.1,129.0,128.6,128.5,127.8,125.6,125.6,43.3,-5.9 .29 \mathrm{Si} \mathrm{NMR}\left(79 \mathrm{MHz}, \mathrm{CDCl}_{3}\right) \delta-11.1$. MALDI $m / z$ calc for $\mathrm{C}_{20} \mathrm{H}_{20} \mathrm{Si}[\mathrm{M}+\mathrm{H}]_{+} .289 .141$. Found 289.153.

Racemic Standard for $\mathbf{4 a}$ :

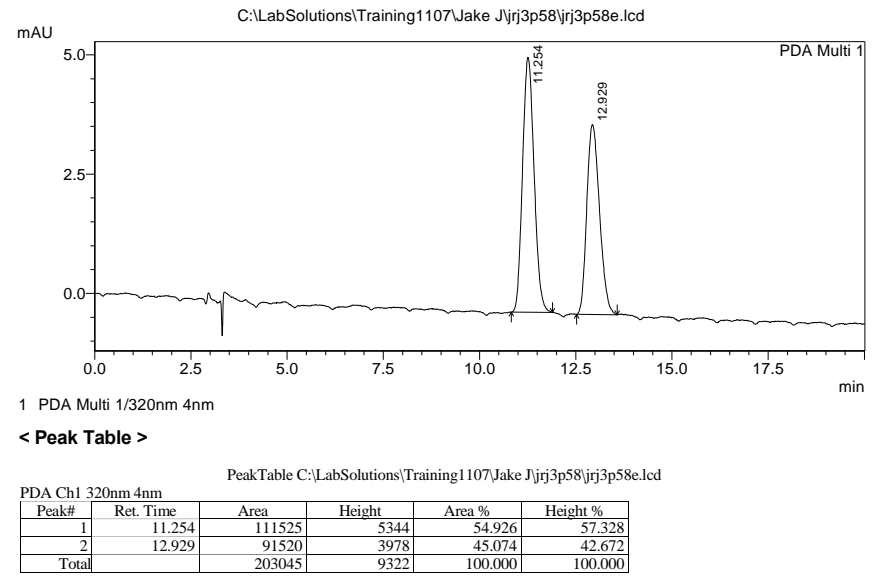

Enantiomerically enriched $(S)$ 4a using $S$-TCPTTL:

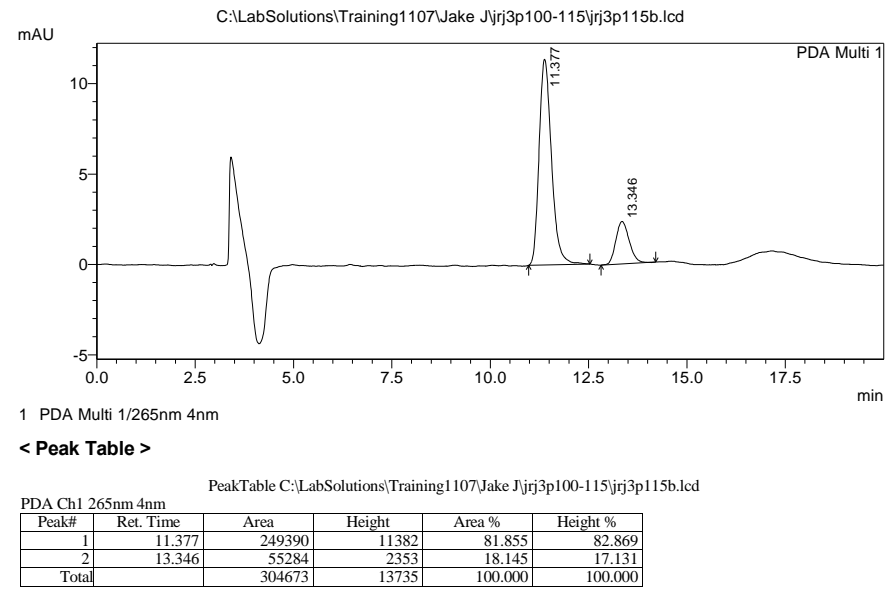




\section{(S)-(bis(4-methoxyphenyl)methyl)(methyl)(phenyl)silane (4b)}

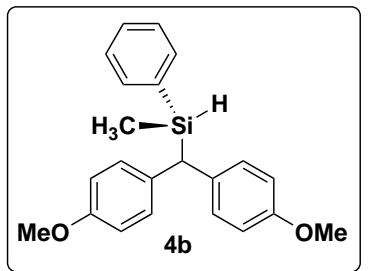

Synthesized using method F with diazo compound $\mathbf{2 b}(0.2 \mathrm{mmol}, 42.0 \mathrm{mg})$ to give a white solid in $45 \%$ yield $(0.09 \mathrm{mmol}, 31.3 \mathrm{mg})$. Enantiomeric ratio was determined by HLPC after hydrolysis with a Daicel CHIRALPAK ® AS column (2\% IPA/ hexanes), $1.0 \mathrm{~mL} / \mathrm{min} . \mathrm{tR}_{\mathrm{R}}(\mathbf{4 b a})=13.7 \mathrm{~min}, \mathrm{tR}(\mathbf{4 b b})=16.2 \mathrm{~min}, 81: 19$ er ( $\mathrm{Si}-\mathrm{OH}$ product). Absolute configuration was assigned to be $(S)$ based on analogy to $\mathbf{5 a}$.

1H NMR (600 MHz, C6D6) $\delta 7.41-7.36(\mathrm{~m}, 2 \mathrm{H}), 7.17$ (m, 2H), $7.15-7.09(\mathrm{~m}, 5 \mathrm{H}), 6.78$ (d, J = 8.6 Hz, 2H), 6.73 $(\mathrm{d}, J=8.7 \mathrm{~Hz}, 2 \mathrm{H}), 4.96(\mathrm{p}, J=3.6 \mathrm{~Hz}, 1 \mathrm{H}), 3.72(\mathrm{~d}, J=3.9 \mathrm{~Hz}, 1 \mathrm{H}), 3.31(\mathrm{~s}, 3 \mathrm{H}), 3.27(\mathrm{~s}, 3 \mathrm{H}), 0.29(\mathrm{~d}, J=3.7 \mathrm{~Hz}$, 3H). ${ }_{13} \mathrm{C}$ NMR $\left(101 \mathrm{MHz}, \mathrm{CDCl}_{3}\right) \delta 157.6,157.5,135.1,135.0,134.8,134.7,129.9,129.8,129.56,127.8,114.0$, 113.9, 55.4, 55.3, 40.9, -5.8. Did not ionize using ESI, MALDI or APCI.

Racemic Standard for $\mathbf{4 b}$ :

<Chromatogram>

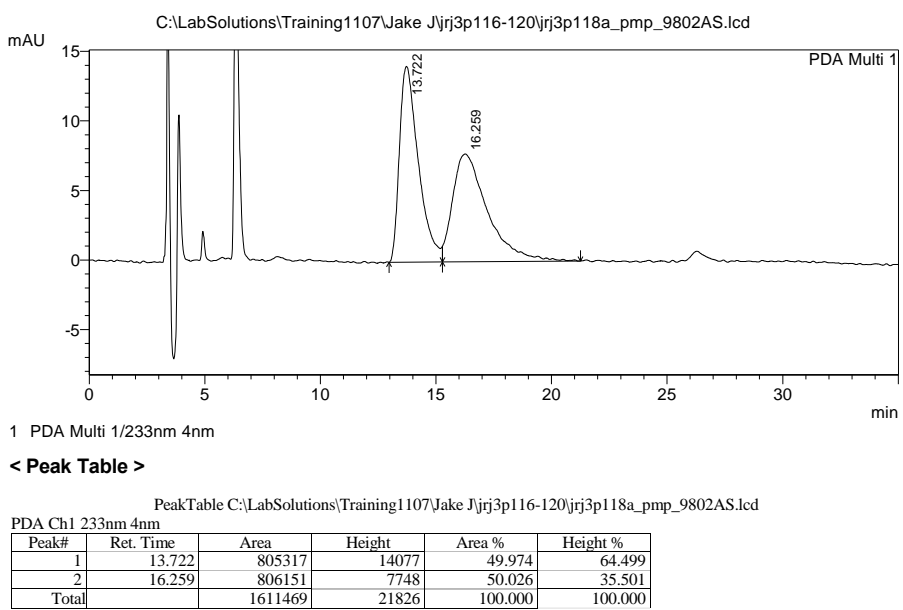

Spectrum index for racemic standard of $\mathbf{4 b}$ :
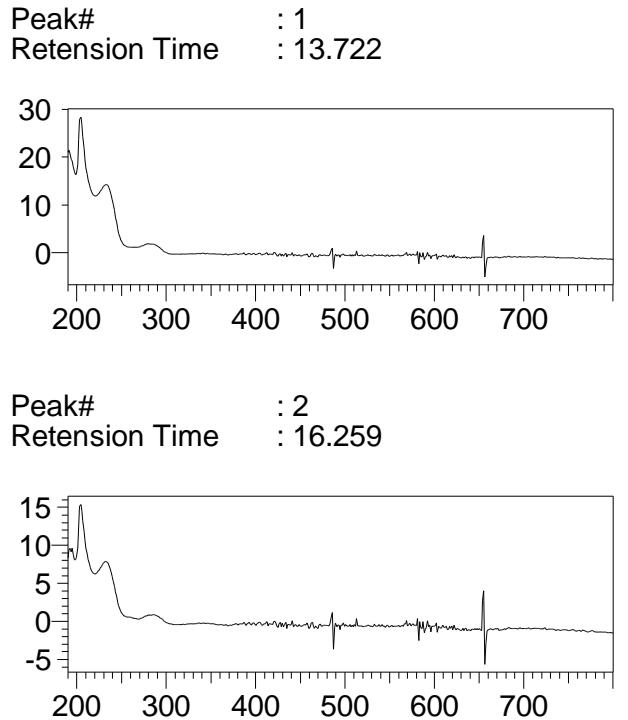
Enantiomerically enriched $(S)$ 4b using $S$-TCPTTL:

$<$ Chromatogram>

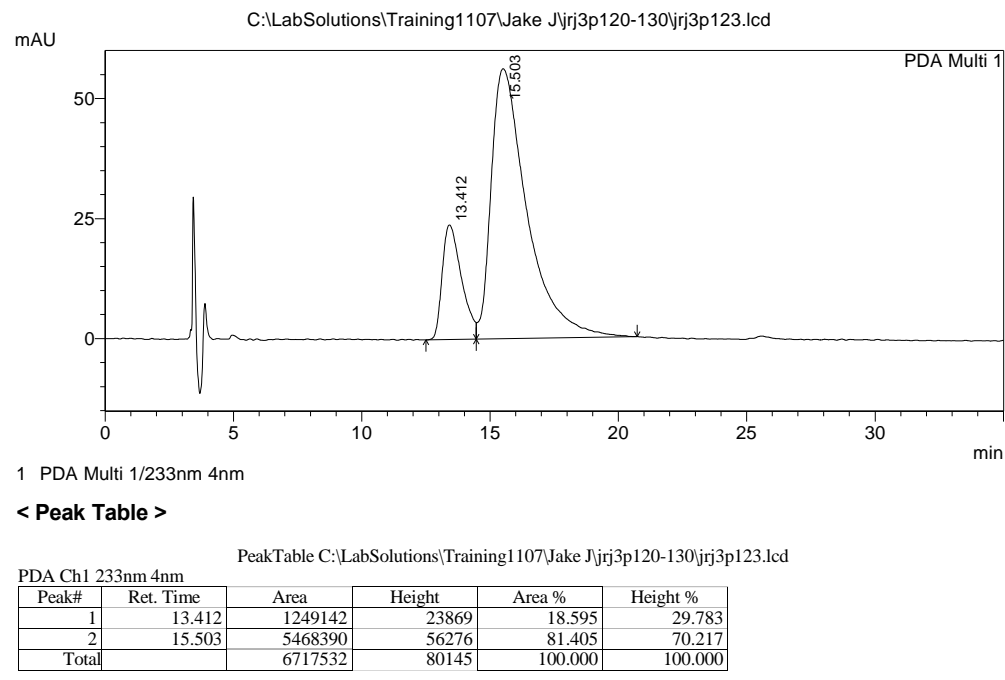

Spectrum index for enantioenriched sample of $\mathbf{4 b}$ :

$\begin{array}{ll}\text { Peak\# } & : 1 \\ \text { Retension Time } & : 13.722\end{array}$
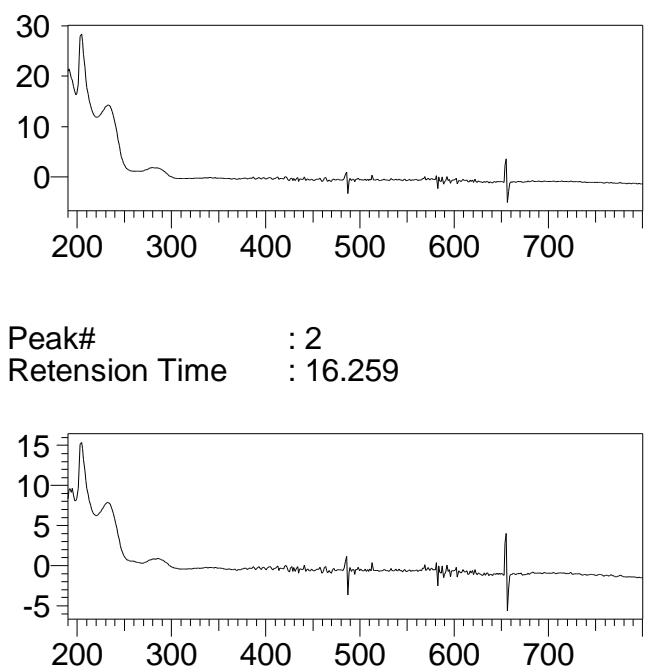

Spectrum indices between racemic standard and enantioenriched are identical, indicating the peaks are identical despite slightly different retention times. 


\section{(S)-(bis(4-chlorophenyl)methyl)(methyl)(phenyl)silane (4c)}

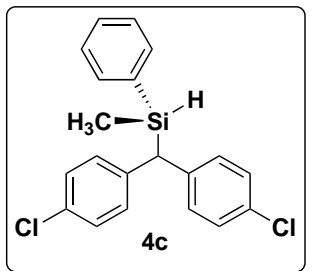

Synthesized using method $F$ with diazo compound $2 \mathrm{c}(0.2 \mathrm{mmol}, 52.6 \mathrm{mg})$ to give a white solid in $91 \%$ yield $(0.158 \mathrm{mmol}, 65.7 \mathrm{mg})$. Enantiomeric ratio was determined by HLPC after hydrolysis with a Daicel CHIRALPAK ® OD-H column $(2 \% \mathrm{IPA} /$ hexanes$), 1.0 \mathrm{~mL} / \mathrm{min} . \mathrm{t}_{\mathrm{R}}(\mathbf{4 c a})=16.4 \mathrm{~min}$, $\mathrm{t}(\mathbf{4 c b})=18.0 \mathrm{~min}, 74: 26 \mathrm{er}$ ( $\mathrm{Si}-\mathrm{OH}$ product). Absolute configuration was assigned to be $(S, S)$ based on analogy to $5 \mathbf{a}$.

1H NMR (600 MHz, CDCl $)$ $\delta 7.40-7.33(\mathrm{~m}, 1 \mathrm{H}), 7.30-7.25(\mathrm{~m}, 4 \mathrm{H}), 7.24(\mathrm{~d}, J=7.3 \mathrm{~Hz}, 2 \mathrm{H}), 7.18(\mathrm{~d}, J=7.3 \mathrm{~Hz}$, 2H), $7.12(\mathrm{~d}, J=7.5 \mathrm{~Hz}, 2 \mathrm{H}), 7.05(\mathrm{~d}, J=7.5 \mathrm{~Hz}, 2 \mathrm{H}), 4.67(\mathrm{p}, J=4.5,4.1 \mathrm{~Hz}, 1 \mathrm{H}), 3.75(\mathrm{~d}, J=3.7 \mathrm{~Hz}, 1 \mathrm{H}), 0.30(\mathrm{~d}$, $J=2.8 \mathrm{~Hz}, 3 \mathrm{H}) .13 \mathrm{C} \mathrm{NMR}\left(101 \mathrm{MHz}, \mathrm{CDCl}_{3}\right) \delta 140.3,140.0,134.8,133.7,131.5,131.4,130.1,130.0,129.9,128.7$, 128.6, 127.9, 41.9, -6.2. $29 \mathrm{Si} \mathrm{NMR}\left(79 \mathrm{MHz}, \mathrm{CDCl}_{3}\right) \delta$-10.8. MALDI $\mathrm{m} / z$ calc for $\mathrm{C}_{20} \mathrm{H}_{18} \mathrm{Cl}_{2} \mathrm{Si}[\mathrm{M}+\mathrm{Na}]+379.045$. Found 379.220.

Racemic Standard for $\mathbf{4 c}$ :

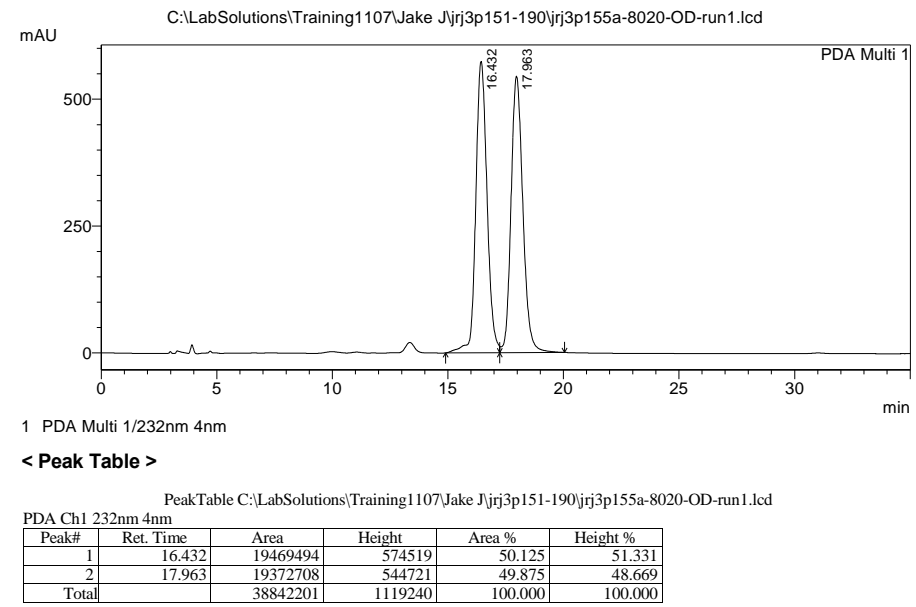

Enantiomerically enriched $(S) \mathbf{4 c}$ using $S$-TCPTTL:

<Chromatogram>

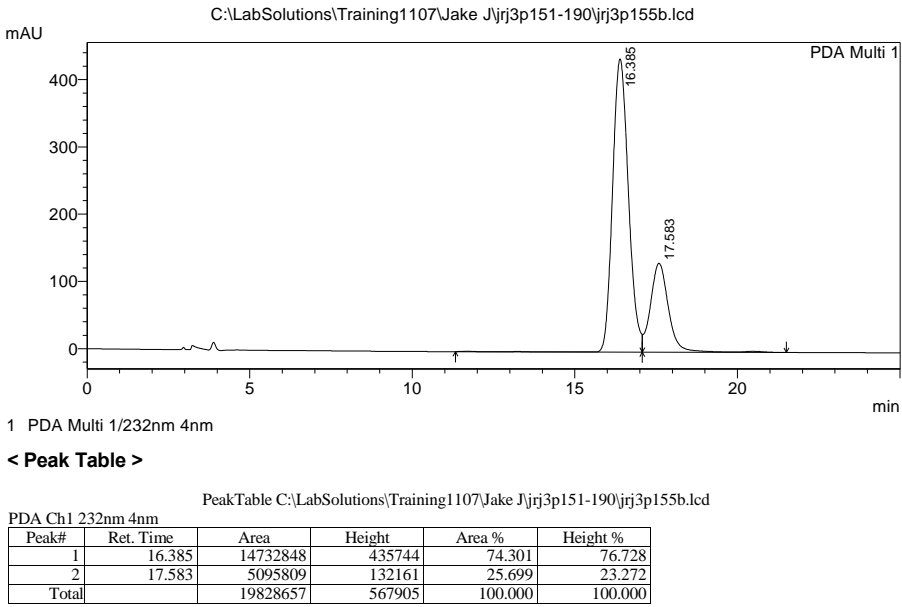




\section{(S)-benzhydryl(4-fluorophenyl)(methyl)silane (4d)}

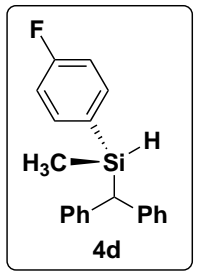

Synthesized using method $\mathrm{F}$ with diazo compound $\mathbf{2 a}(0.2 \mathrm{mmol}, 38.8 \mathrm{mg})$ to give a white solid in $66 \%$ yield $(0.132 \mathrm{mmol}, 40.4 \mathrm{mg})$. Enantiomeric ratio was determined by HLPC after hydrolysis with a Daicel CHIRALPAK ® AD-H column (2\% IPA/ hexanes), 1.0 $\mathrm{mL} / \mathrm{min} . \mathrm{t}_{\mathrm{R}}(\mathbf{4 d a})=10.2 \mathrm{~min}, \mathrm{tR}(\mathbf{4 d b})=12.9 \mathrm{~min} 80: 20 \mathrm{er}(\mathrm{Si}-\mathrm{OH}$ product $)$. Absolute configuration was assigned to be $(S)$ based on analogy to $\mathbf{5 a}$.

${ }_{1} \mathrm{H}$ NMR $\left(400 \mathrm{MHz}_{\mathrm{CDCl}}\right) \delta 7.31(\mathrm{dd}, J=9.4,5.8 \mathrm{~Hz}, 3 \mathrm{H}), 7.28-7.23(\mathrm{~m}, 5 \mathrm{H}), 7.23-7.13(\mathrm{~m}, 4 \mathrm{H}), 6.99(\mathrm{t}, J=$ $8.7 \mathrm{~Hz}, 2 \mathrm{H}), 4.76(\mathrm{p}, J=3.9 \mathrm{~Hz}, 1 \mathrm{H}), 3.82(\mathrm{~d}, J=3.9 \mathrm{~Hz}, 1 \mathrm{H}), 0.35(\mathrm{~d}, J=3.6 \mathrm{~Hz}, 3 \mathrm{H}) .13 \mathrm{C} \mathrm{NMR}(101 \mathrm{MHz}, \mathrm{CDCl} 3)$ $\delta 164.0\left(\mathrm{~d}, J_{C F}=249.0 \mathrm{~Hz}\right), 141.9,141.75,136.8\left(\mathrm{~d}, J_{C C C F}=7.4 \mathrm{~Hz}\right), 130.1\left(\mathrm{~d}, J_{C C C C F}=3.9 \mathrm{~Hz}\right), 128.9,128.8,128.6$, 128.4, 125.6, 125.6, $115.0\left(\mathrm{~d}, J_{C C F}=19.8 \mathrm{~Hz}\right), 43.2,-5.9 .19 \mathrm{~F} \mathrm{NMR}\left(376 \mathrm{MHz}, \mathrm{CDCl}_{3}\right) \delta-110.96 .29 \mathrm{Si} \mathrm{NMR}(79 \mathrm{MHz}$, $\left.\mathrm{CDCl}_{3}\right) \delta$-11.1. MALDI $m / z$ calc for $\mathrm{C}_{20} \mathrm{H}_{19} \mathrm{FSi}[\mathrm{M}+\mathrm{Na}]+329.113$. Found 329.158.

Racemic Standard for $\mathbf{4 d}$ :

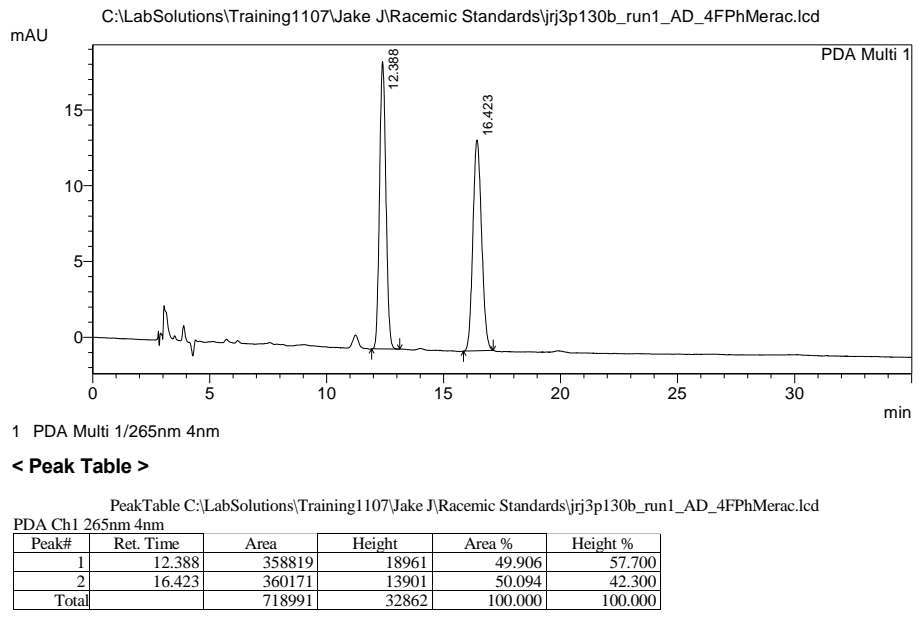

Spectrum index for racemic standard of $\mathbf{4 d}$ :
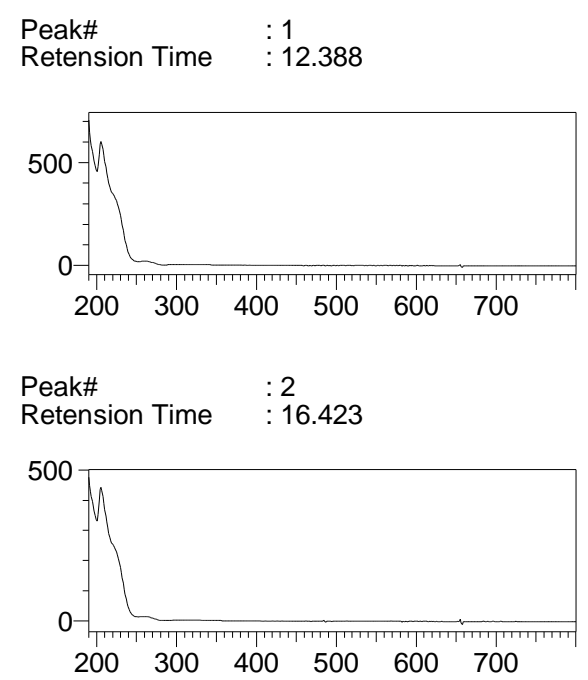
Enantiomerically enriched ( $S$ ) 4d using $S$-TCPTTL:

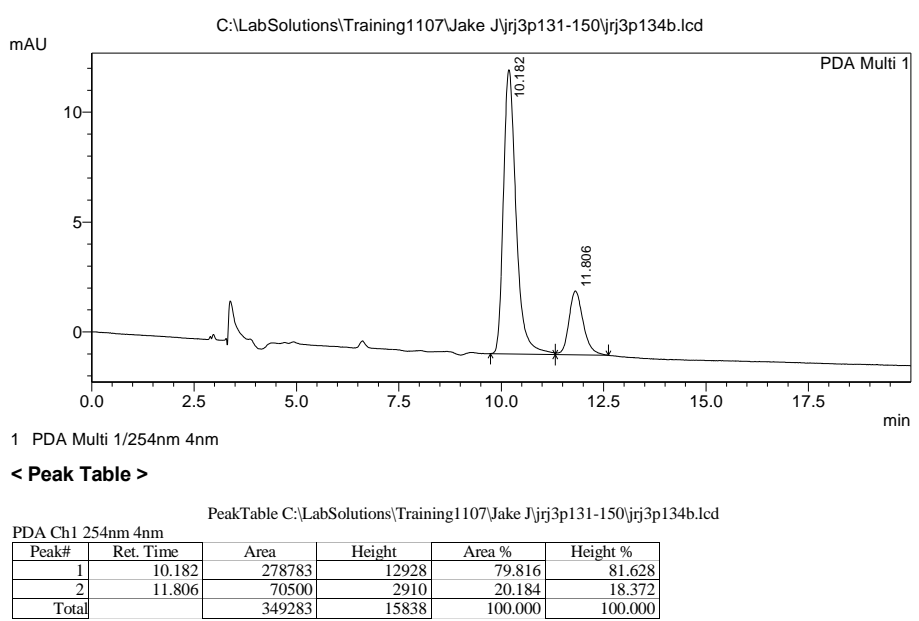

Spectrum index for enantioenriched sample of $\mathbf{4 d}$ :

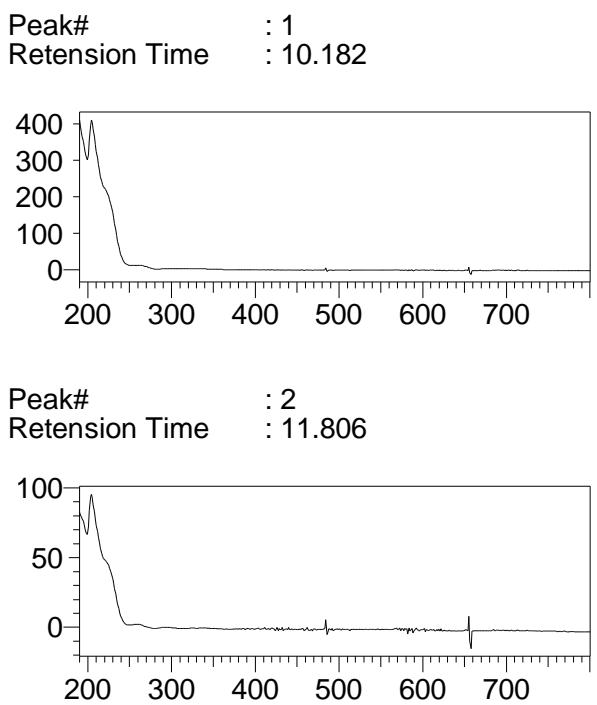

Spectrum indices between racemic standard and enantioenriched are identical, indicating the peaks are identical despite slightly different retention times. 


\section{(S)-benzhydryl(4-methoxyphenyl)(methyl)silane (4e)}

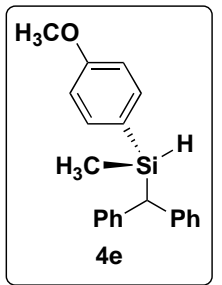

Synthesized using method $\mathrm{F}$ with diazo compound $\mathbf{2 a}(0.2 \mathrm{mmol}, 42.0 \mathrm{mg})$ to give a white solid in $72 \%$ yield $(0.144 \mathrm{mmol}, 45.9 \mathrm{mg})$. Enantiomeric ratio was determined by HLPC after hydrolysis with a Daicel CHIRALPAK ${ }^{\circledR}$ OD-H column (1\% IPA/ hexanes), 1.0 $\mathrm{mL} / \mathrm{min}$. $\mathrm{tR}_{\mathrm{R}}(\mathbf{4 e a})=17.2, \mathrm{t}_{\mathrm{R}}(\mathbf{4 e b})=18.7,50: 50 \mathrm{er}(\mathrm{Si}-\mathrm{OH}$ product $)$. Absolute configuration was assigned to be $(S, S)$ based on analogy to $\mathbf{5 a}$.

${ }_{1} \mathrm{H}$ NMR $\left(600 \mathrm{MHz}_{\mathrm{CDCl}}\right) \delta 7.31-7.08(\mathrm{~m}, 13 \mathrm{H}), 6.81(\mathrm{~d}, J=8.5 \mathrm{~Hz}, 2 \mathrm{H}), 4.70(\mathrm{p}, J=3.7 \mathrm{~Hz}, 1 \mathrm{H}), 3.79(\mathrm{~d}, J=$ $1.6 \mathrm{~Hz}, 4 \mathrm{H}), 0.28(\mathrm{~d}, J=3.6 \mathrm{~Hz}, 3 \mathrm{H}) .{ }_{13} \mathrm{C} \mathrm{NMR}\left(151 \mathrm{MHz}, \mathrm{CDCl}_{3}\right) \delta 160.7,142.3,142.0,141.4,136.8,136.3,129.0$, 128.8, 125.4, 125.3, 113.5, 113.1, 55.7, 43.4, -7.3. 29 Si NMR (79 MHz, CDCl $) \delta-11.6$. MALDI $m / z$ calc for $\mathrm{C}_{21} \mathrm{H}_{22} \mathrm{OSi}$ $[\mathrm{M}+\mathrm{H}]+319.151$. Found 319.245.

Racemic Standard for $\mathbf{4 e}$ :

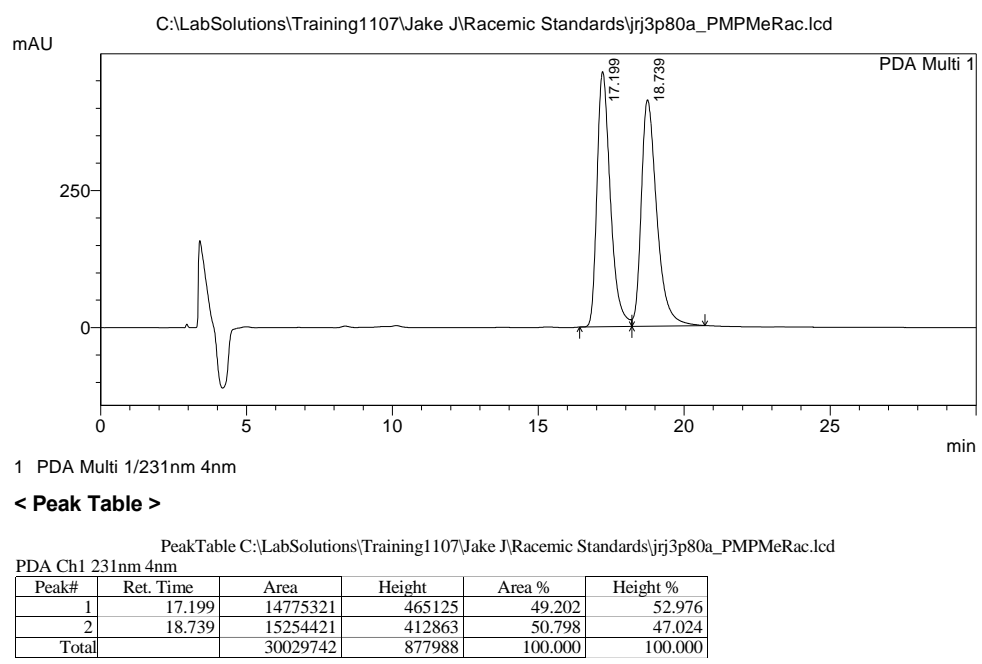

HPLC trace of $4 \mathbf{e}$ using $S$-TCPTTL:

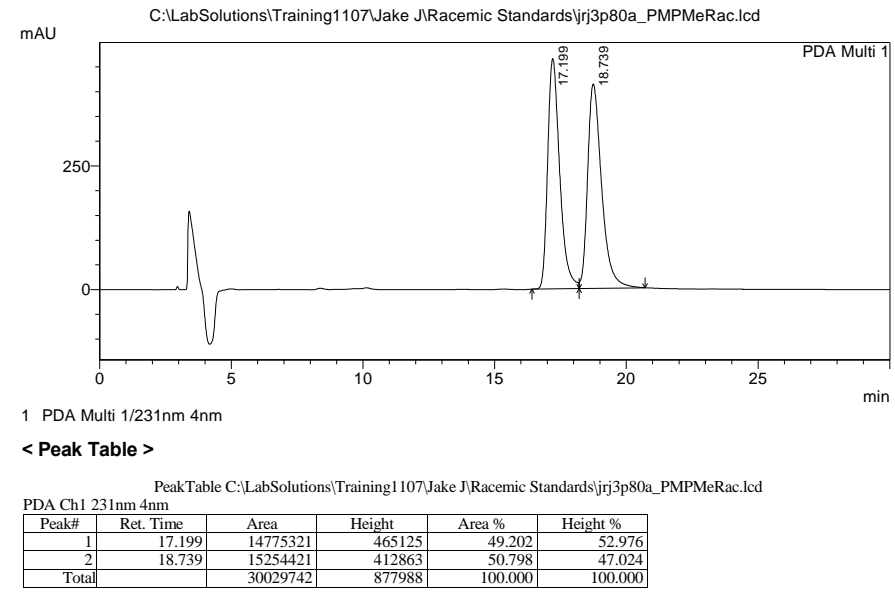




\section{(S)-benzhydryl(4-methylphenyl)(methyl)silane (4f)}

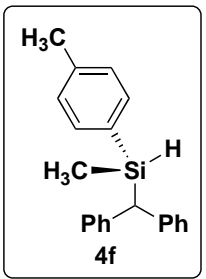

Synthesized using method $\mathrm{F}$ with diazo compound $\mathbf{2 a}(0.2 \mathrm{mmol}, 38.8 \mathrm{mg})$ to give a white solid in $76 \%$ yield $(0.152 \mathrm{mmol}, 46.0 \mathrm{mg})$. Enantiomeric ratio was determined by HLPC after hydrolysis with a Daicel CHIRALPAK $®$ AD-H column (2\% IPA/ hexanes), 1.0 $\mathrm{mL} / \mathrm{min}$. tR $(\mathbf{4 f a})=9.9 \mathrm{~min}, \mathrm{tR}(\mathbf{4 f b})=10.7 \mathrm{~min}, 74: 26$ er $(\mathrm{Si}-\mathrm{OH}$ product $)$. Absolute configuration was assigned to be $(S)$ based on analogy to $5 \mathbf{a}$.

${ }_{1} \mathrm{H} \mathrm{NMR}\left(600 \mathrm{MHz}, \mathrm{CDCl}_{3}\right) \delta 7.31-7.24(\mathrm{~m}, 5 \mathrm{H}), 7.22(\mathrm{dd}, J=8.8,2.4 \mathrm{~Hz}, 5 \mathrm{H}), 7.20-7.12(\mathrm{~m}, 2 \mathrm{H}), 7.11(\mathrm{~d}, J=$ $7.5 \mathrm{~Hz}, 2 \mathrm{H}), 4.73(\mathrm{p}, J=3.8 \mathrm{~Hz}, 1 \mathrm{H}), 3.83(\mathrm{~d}, J=3.9 \mathrm{~Hz}, 1 \mathrm{H}), 2.34(\mathrm{~s}, 3 \mathrm{H}), 0.31(\mathrm{~d}, J=3.7 \mathrm{~Hz}, 3 \mathrm{H}) .13 \mathrm{C} \mathrm{NMR}(151$ $\left.\mathrm{MHz}_{2} \mathrm{CDCl}_{3}\right) \delta 142.4,142.2,139.5,135.1,131.0,129.1,129.0,128.7,128.6,128.5,125.5,44.2,22.1,-5.8 .29 \mathrm{Si}$ NMR $\left(79 \mathrm{MHz}, \mathrm{CDCl}_{3}\right) \delta$-11.3. MALDI $\mathrm{m} / z$ calc for $\mathrm{C}_{21} \mathrm{H}_{22} \mathrm{Si}[\mathrm{M}+\mathrm{H}]+328.138$. Found 325.207.

Racemic Standard for $\mathbf{4 f}$ :

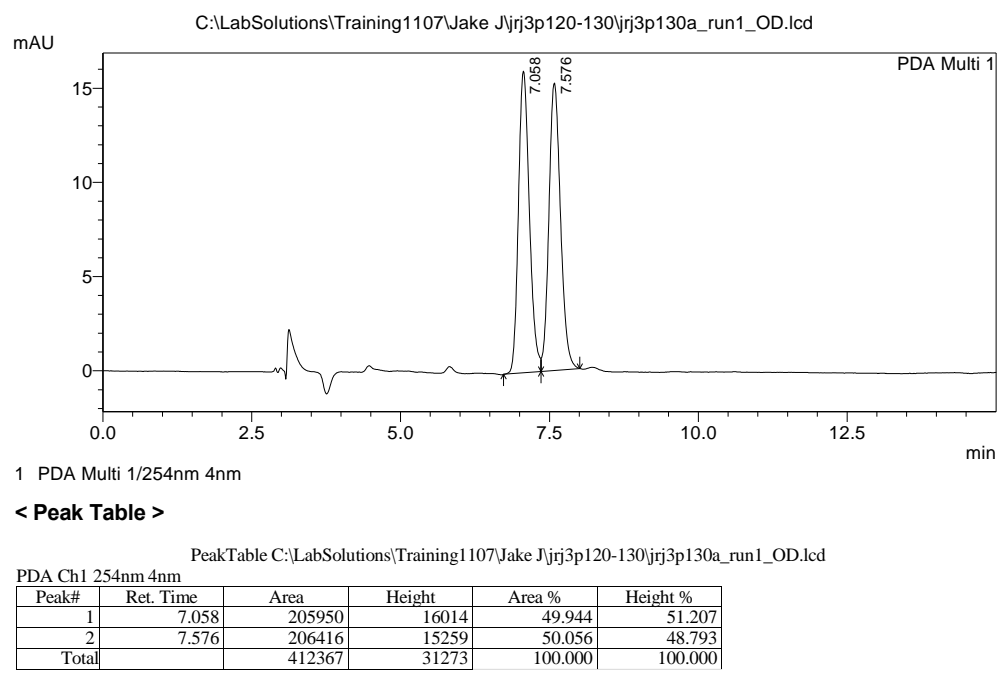

Enantiomerically enriched $(S) \mathbf{4 f}$ using $S$-TCPTTL:
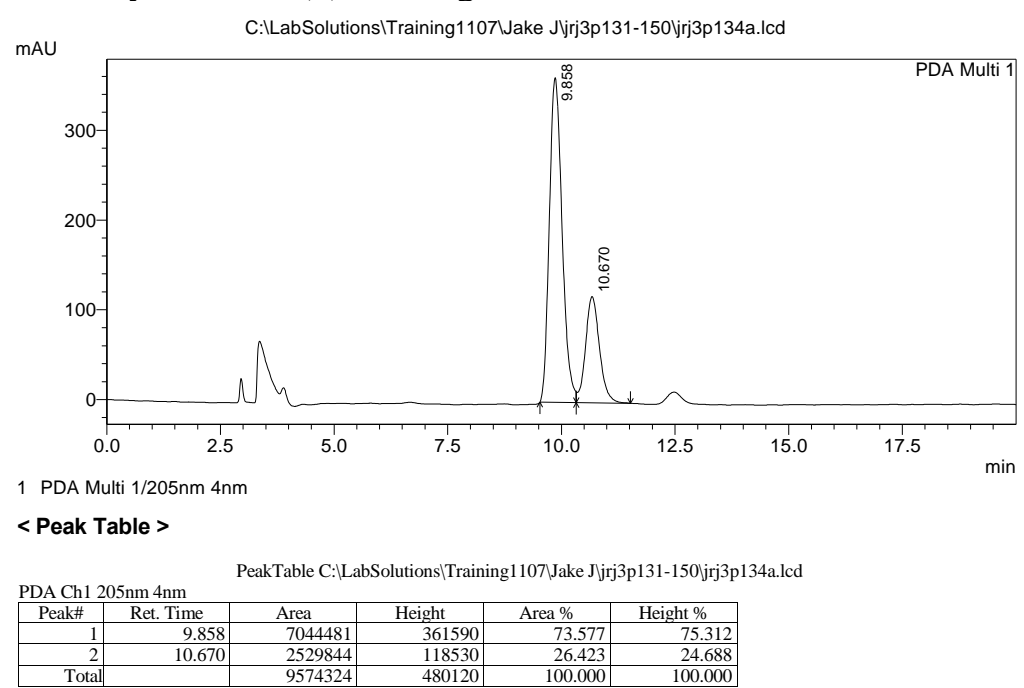


\section{(S)-benzhydryl(methyl)(naphthalen-2-yl)silane (4g)}

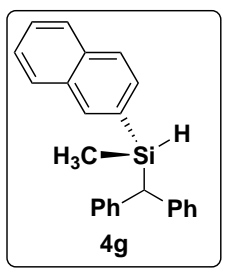

Synthesized according to the general procedure for enantioselective donor/donor insertion using 2a $(0.2 \mathrm{mmol}, 38.8 \mathrm{mg})$ to give a white solid in $69 \%$ yield $(0.158 \mathrm{mmol}, 46.7 \mathrm{mg})$. Enantiomeric ratio was determined by HLPC after hydrolysis with a Daicel CHIRALPAK ( $\mathrm{AD}-\mathrm{H}$ column (1\% IPA/ hexanes), $1.0 \mathrm{~mL} / \mathrm{min} . \mathrm{t}_{\mathrm{R}}(\mathbf{4 g a})=19.5 \mathrm{~min}, \mathrm{tR}(\mathbf{4 g b})=24.2$ min, 82:18 er ( $\mathrm{Si}-\mathrm{OH}$ product). Absolute configuration was assigned to be $(S)$ based on analogy to $\mathbf{5 a}$.

1H NMR (600 MHz, C6 6 6) $7.86(\mathrm{~s}, 1 \mathrm{H}), 7.61-7.49(\mathrm{~m}, 3 \mathrm{H}), 7.33(\mathrm{~d}, J=8.1 \mathrm{~Hz}, 1 \mathrm{H}), 7.28-7.18(\mathrm{~m}, 6 \mathrm{H}), 7.13(\mathrm{t}$, $J=7.6 \mathrm{~Hz}, 2 \mathrm{H}), 7.04(\mathrm{dt}, J=13.7,7.4 \mathrm{~Hz}, 3 \mathrm{H}), 6.97(\mathrm{t}, J=7.3 \mathrm{~Hz}, 1 \mathrm{H}), 5.04(\mathrm{p}, J=3.6 \mathrm{~Hz}, 1 \mathrm{H}), 3.83(\mathrm{~d}, J=3.8 \mathrm{~Hz}$, $1 \mathrm{H}), 0.32(\mathrm{~d}, J=3.6 \mathrm{~Hz}, 3 \mathrm{H}) .{ }_{13} \mathrm{C}$ NMR $\left(151 \mathrm{MHz}, \mathrm{CDCl}_{3}\right) \delta 142.2,142.0,136.1,134.0,132.9,132.3,130.9,129.1$, 129.0, 128.66, 128.5, 128.2, 127.8, 127.0, 126.7, 126.0, 125.7, 125.6, 43.3, -5.7. 29Si NMR (119 MHz, C6 6 6) $\delta-10.9$ MALDI $m / z$ calc for $\mathrm{C}_{24} \mathrm{H}_{22} \mathrm{Si}[\mathrm{M}+\mathrm{Na}]+361.1383$. Found 363.1318.

Racemic Standard for $\mathbf{4 g}$ :

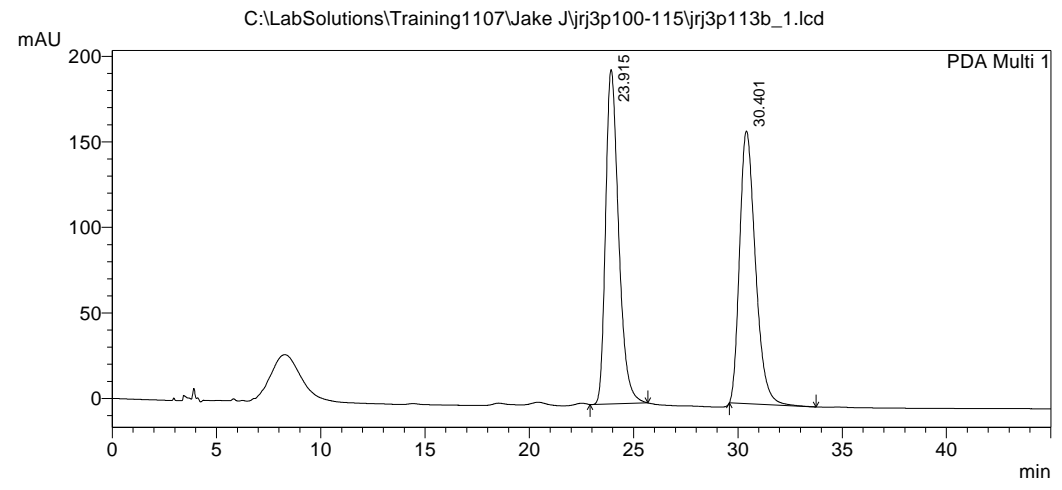

Enantiomerically enriched $(S) \mathbf{4 g}$ using $S$-TCPTTL:

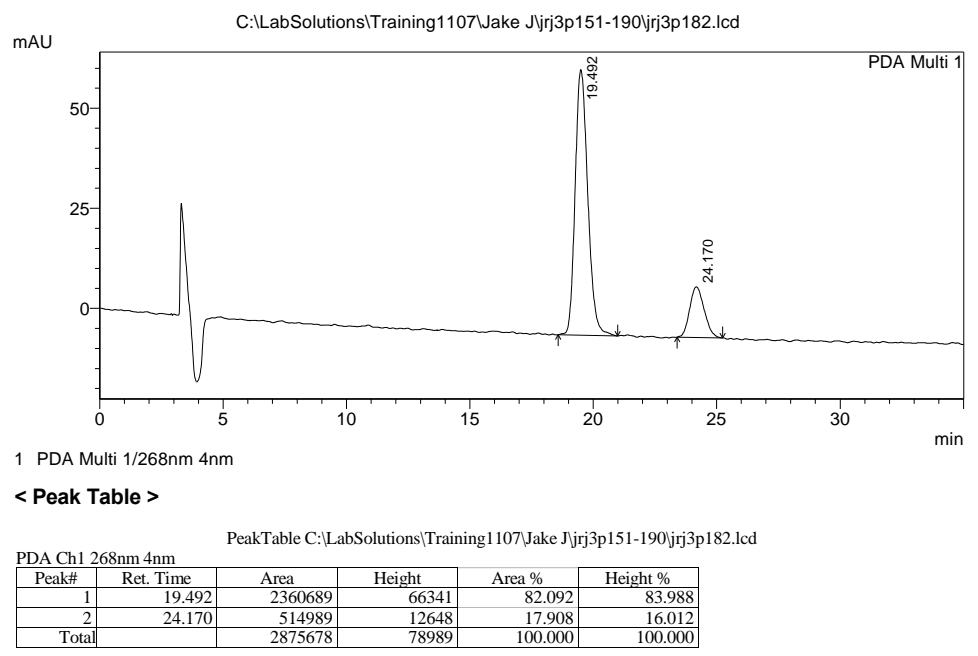




\section{(S)-benzhydryl(methyl)(naphthalen-1-yl)silane (4h)}

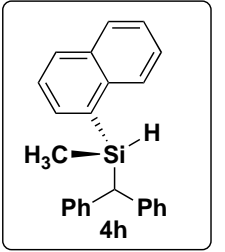

Synthesized using method $F$ with diazo compound $2 \mathbf{a}(0.2 \mathrm{mmol}, 38.8 \mathrm{mg})$ to give a white solid in $60 \%$ yield $(0.12 \mathrm{mmol}, 40.6 \mathrm{mg})$. Enantiomeric ratio was determined by HLPC after hydrolysis with a Daicel CHIRALPAK ® AD-H column (1\% IPA/ hexanes), 1.0 $\mathrm{mL} / \mathrm{min}$. tR $(\mathbf{4 h a})=10.1 \mathrm{~min}$, tR $(\mathbf{4 h b})=11.2 \mathrm{~min}, 77: 23 \mathrm{er}(\mathrm{Si}-\mathrm{OH}$ product $)$. Absolute configuration was assigned to be $(S, S)$ based on analogy to $\mathbf{5 a}$.

${ }_{1} \mathrm{H}$ NMR $\left(600 \mathrm{MHz}, \mathrm{C}_{6} \mathrm{D}_{6}\right) \delta 8.08-8.01(\mathrm{~m}, 1 \mathrm{H}), 7.63-7.58(\mathrm{~m}, 2 \mathrm{H}), 7.54-7.49(\mathrm{~m}, 1 \mathrm{H}), 7.25(\mathrm{~m}, 2 \mathrm{H}), 7.19(\mathrm{~d}, J$ $=7.5 \mathrm{~Hz}, 4 \mathrm{H}), 7.14(\mathrm{~s}, 1 \mathrm{H}), 7.09(\mathrm{t}, J=7.6 \mathrm{~Hz}, 2 \mathrm{H}), 7.03-6.96(\mathrm{~m}, 3 \mathrm{H}), 6.91(\mathrm{t}, J=7.3 \mathrm{~Hz}, 1 \mathrm{H}), 5.33(\mathrm{p}, J=3.6 \mathrm{~Hz}$, $1 \mathrm{H}), 4.05(\mathrm{~d}, J=4.0 \mathrm{~Hz}, 1 \mathrm{H}), 0.36(\mathrm{~d}, J=3.7 \mathrm{~Hz}, 3 \mathrm{H}) .13 \mathrm{C}$ NMR $\left(151 \mathrm{MHz}, \mathrm{CDCl}_{3}\right) \delta 142.3,142.2,137.1,135.2$, 133.3, 133.3, 130.4, 129.0, 129.0, 128.6, 128.4, 127.8, 126.0, 125.6, 125.5, 125.1, 43.1, -5.0. 29Si NMR (79 MHz, $\left.\mathrm{CDCl}_{3}\right) \delta$-15.6. MALDI $\mathrm{m} / \mathrm{z}$ calc for $\mathrm{C}_{24} \mathrm{H}_{22} \mathrm{Si}[\mathrm{M}+\mathrm{Na}]+361.1383$. Found 361.1446 .

Racemic Standard for $\mathbf{4 h}$ :

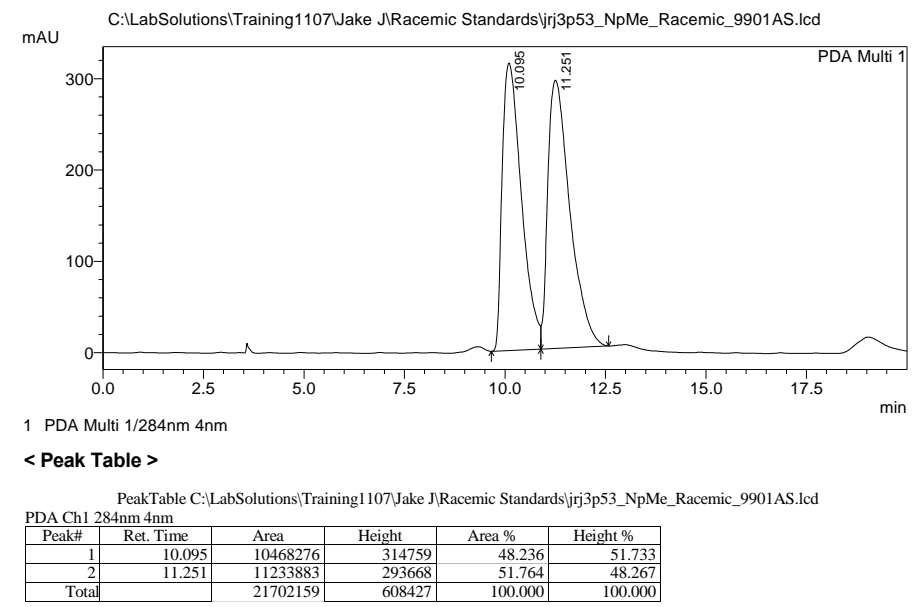

Spectrum index for racemic standard of $\mathbf{4 h}$ :

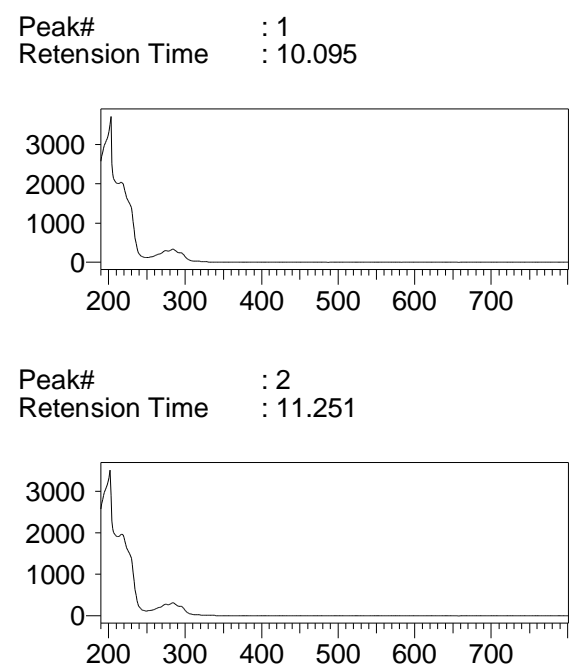


Enantiomerically enriched $(S)$ 4h using $S$-TCPTTL:

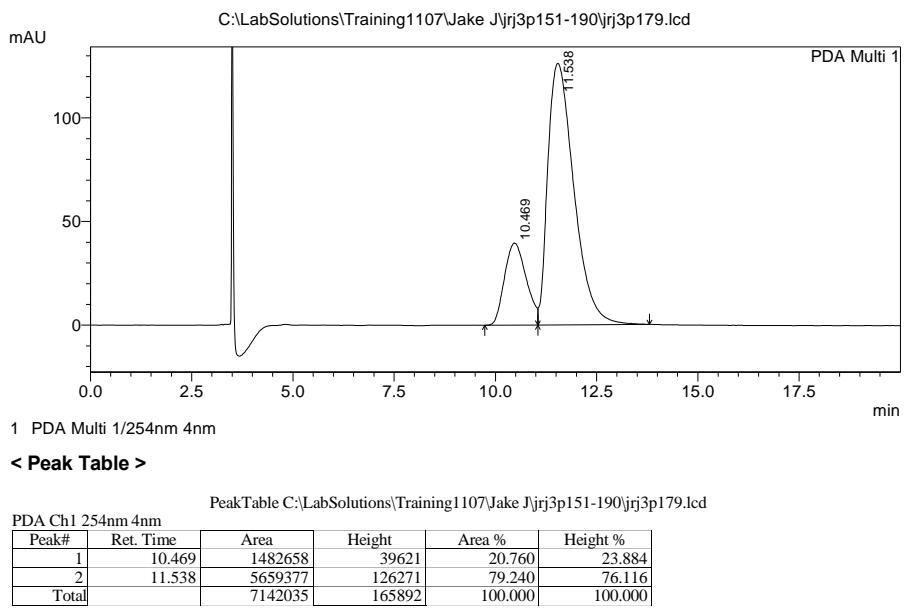

Spectrum index for enantioenriched sample of $\mathbf{4 h}$ :

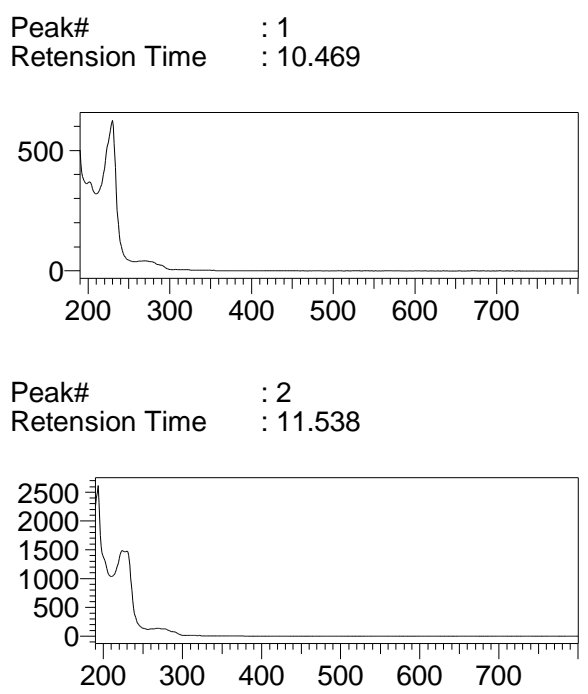

Spectrum indices between racemic standard and enantioenriched are identical, indicating the peaks are identical despite slightly different retention times. 


\section{(S)-benzhydryl(2-methylphenyl)(methyl)silane (4i)}

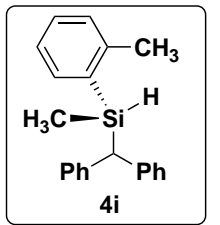

Synthesized using method $F$ with diazo compound $\mathbf{2 a}(0.2 \mathrm{mmol}, 38.8 \mathrm{mg})$ to give a white solid in $55 \%$ yield $(0.110 \mathrm{mmol}, 33.2 \mathrm{mg})$. Enantiomeric ratio was determined by HLPC after hydrolysis with a Daicel CHIRALPAK ® OD-H column (1\% IPA/ hexanes), 1.0 $\mathrm{mL} / \mathrm{min}$. $\mathrm{t}(\mathbf{4 i a})=10.9 \mathrm{~min}, \mathrm{tR}(4 \mathbf{i b})=12.6 \mathrm{~min}, 69: 31 \mathrm{er}(\mathrm{Si}-\mathrm{OH}$ product $)$. Absolute configuration was assigned to be $(S, S)$ based on analogy to $\mathbf{5 a}$.

1H NMR (600 MHz, $\left.\mathrm{CDCl}_{3}\right) \delta 7.34(\mathrm{~m}, 1 \mathrm{H}), 7.27(\mathrm{~m}, 1 \mathrm{H}), 7.25-7.21(\mathrm{~m}, 4 \mathrm{H}), 7.15(\mathrm{~m}, 5 \mathrm{H}), 7.11-7.04(\mathrm{~m}, 3 \mathrm{H})$, $4.82(\mathrm{p}, J=3.7 \mathrm{~Hz}, 1 \mathrm{H}), 3.86(\mathrm{~d}, J=4.3 \mathrm{~Hz}, 1 \mathrm{H}), 2.17(\mathrm{~s}, 3 \mathrm{H}), 0.28(\mathrm{~d}, J=3.7 \mathrm{~Hz}, 3 \mathrm{H}) .13 \mathrm{C} \mathrm{NMR}\left(151 \mathrm{MHz}, \mathrm{CDCl}_{3}\right)$ $\delta$ 144.3, 142.4, 142.3, 135.6, 133.8, 129.8, 129.7, 129.1, 128.8, 128.6, 128.4, 125.6, 125.5, 125.0, 43.2, 22.6, -5.4. ${ }_{29} \mathrm{Si}$ NMR (119 MHz, $\left.\mathrm{CDCl}_{3}\right) \delta-13.7 \mathrm{MALDI} m / z$ calc for $\mathrm{C}_{21} \mathrm{H}_{22} \mathrm{Si}[\mathrm{M}+\mathrm{H}]+328.138$. Found 325.227.

Racemic Standard for 4i:

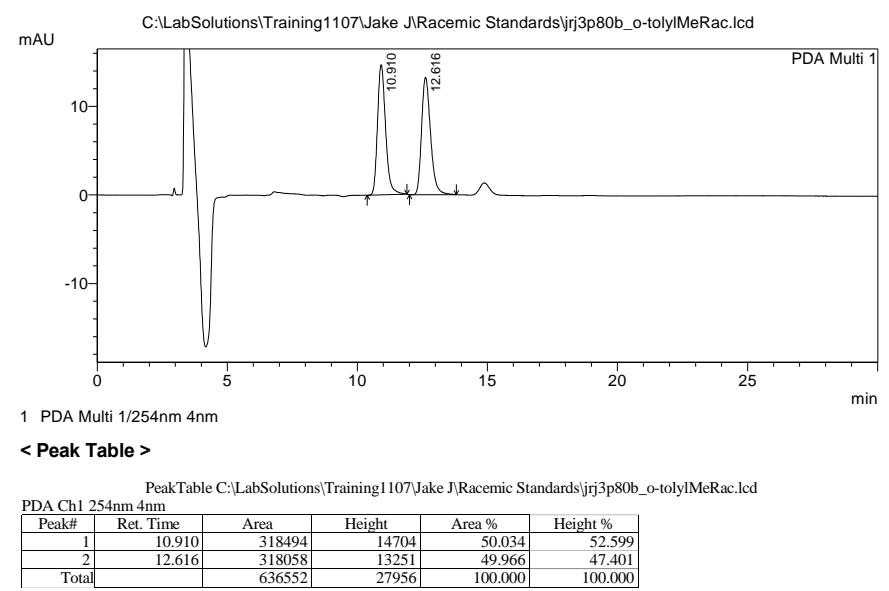

Enantiomerically enriched $(S, S)$ 4i using $S$-TCPTTL:

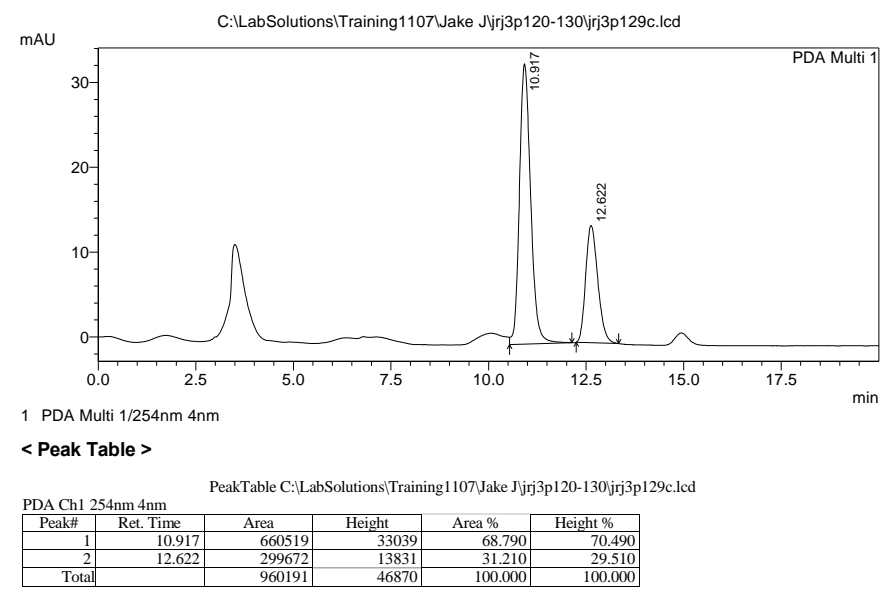




\section{(S)-benzhydryl(mesityl)(methyl)silane (4j)}

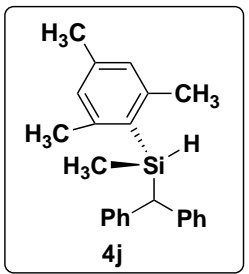

Synthesized using method $\mathrm{F}$ with diazo compound $\mathbf{2 a}(0.2 \mathrm{mmol}, 38.8 \mathrm{mg})$ to give a white solid in $64 \%$ yield $(0.128 \mathrm{mmol}, 42.3 \mathrm{mg})$. Enantiomeric ratio was determined by HLPC after hydrolysis with a Daicel CHIRALPAK ® OD-H column (2\% IPA/ hexaness $), 1.0 \mathrm{~mL} / \mathrm{min}$. tR $(\mathbf{4 j a})=6.8 \mathrm{~min}$, tR $(\mathbf{4 j b})=8.6 \mathrm{~min}, 60: 40 \mathrm{er}(\mathrm{Si}-\mathrm{OH}$ product$)$. Absolute configuration was assigned to be $(S)$ based on analogy to $\mathbf{5 a}$.

${ }_{1} \mathrm{H}$ NMR $\left(400 \mathrm{MHz}, \mathrm{CDCl}_{3}\right) \delta 7.31(\mathrm{~m}, 3 \mathrm{H}), 7.28-7.13(\mathrm{~m}, 2 \mathrm{H}), 7.10(\mathrm{~d}, J=4.7 \mathrm{~Hz}, 4 \mathrm{H}), 7.04(\mathrm{~m}, 1 \mathrm{H}), 6.75(\mathrm{~s}, 2 \mathrm{H})$, $4.94(\mathrm{p}, J=4.3 \mathrm{~Hz}, 1 \mathrm{H}), 3.92$ (d, $J=5.4 \mathrm{~Hz}, 1 \mathrm{H}), 2.24(\mathrm{~s}, 9 \mathrm{H}), 0.29$ (d, J=4.0 Hz, 3H). 13C NMR (101 MHz, CDCl 3$)$ $\delta 144.7,142.7,142.4,139.4,129.3,129.1,128.7,128.6,128.4,128.3,125.7,125.3,43.4,24.1,21.2,-3.7 .29 \mathrm{Si}$ NMR $\left(79 \mathrm{MHz}, \mathrm{CDCl}_{3}\right) \delta$-18.0. MALDI $m / z$ calc for $\mathrm{C}_{23} \mathrm{H}_{26} \mathrm{Si}[\mathrm{M}+\mathrm{Na}]+353.170$. Found 353.248.

Racemic Standard for $\mathbf{4 j}$ :

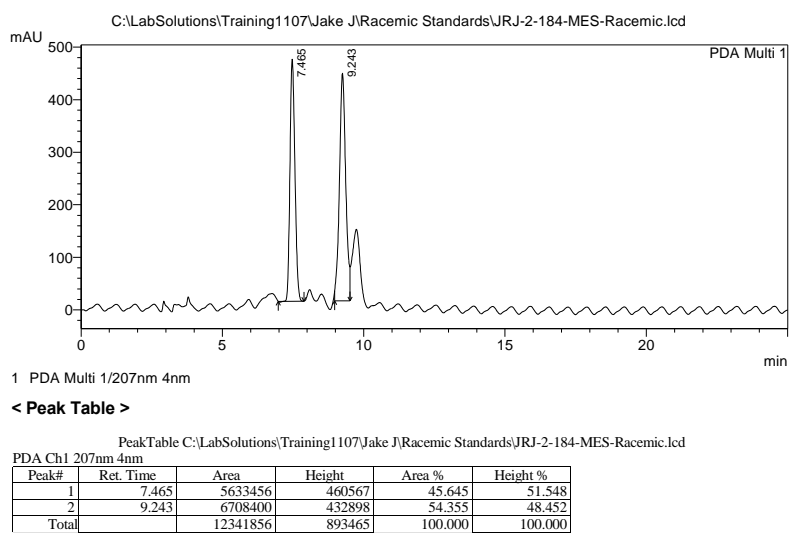

Enantiomerically enriched $(S, S) 4 \mathbf{j}$ using $S$-TCPTTL:

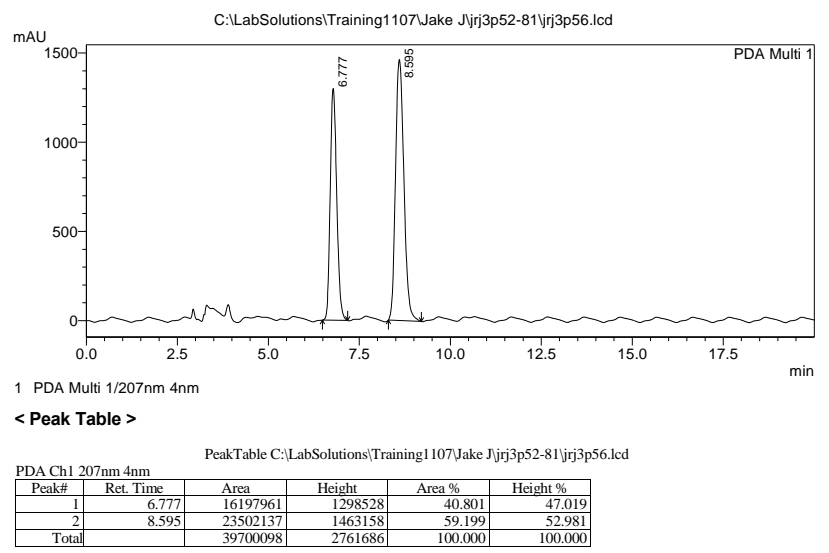




\section{(S)-benzhydryl(isobutyl)(naphthalen-1-yl)silane (4k)}

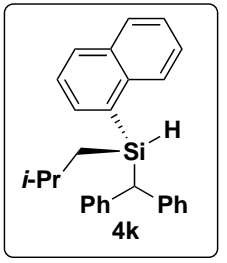

Synthesized using method $\mathrm{F}$ with diazo compound $\mathbf{2 a}(0.2 \mathrm{mmol}, 38.8 \mathrm{mg})$ to give a white solid in $60 \%$ yield $(0.120 \mathrm{mmol}, 41.3 \mathrm{mg})$. Enantiomeric ratio was determined by HLPC after hydrolysis with a Daicel CHIRALPAK ® AD-H column (1\% IPA/ hexanes), $1.0 \mathrm{~mL} / \mathrm{min}$. $\mathrm{tR}(\mathbf{4 k a})=10.9 \mathrm{~min}, \mathrm{tR}(\mathbf{4 k b})=12.6 \mathrm{~min}, 82: 18 \mathrm{er}(\mathrm{Si}-\mathrm{OH}$ product $)$. Absolute configuration was assigned to be $(S)$ based on analogy to $\mathbf{5 a}$.

1H NMR (600 MHz, CDCl $) \delta 7.92(\mathrm{~d}, J=8.3 \mathrm{~Hz}, 1 \mathrm{H}), 7.83(\mathrm{dd}, J=8.1,6.4 \mathrm{~Hz}, 2 \mathrm{H}), 7.59(\mathrm{dd}, J=6.7,1.2 \mathrm{~Hz}, 1 \mathrm{H})$, $7.48-7.43(\mathrm{~m}, 1 \mathrm{H}), 7.40(\mathrm{ddd}, J=8.1,6.8,1.4 \mathrm{~Hz}, 1 \mathrm{H}), 7.36(\mathrm{td}, J=7.3,6.7,1.2 \mathrm{~Hz}, 1 \mathrm{H}), 7.30-7.26(\mathrm{~m}, 5 \mathrm{H}), 7.26$ $-7.23(\mathrm{~m}, 1 \mathrm{H}), 7.17(\mathrm{ddd}, J=8.5,5.1,1.8 \mathrm{~Hz}, 1 \mathrm{H}), 7.09(\mathrm{~d}, J=6.8 \mathrm{~Hz}, 2 \mathrm{H}), 7.07-7.03(\mathrm{~m} \mathrm{2H}), 7.00(\mathrm{td}, J=6.9,1.4$ $\mathrm{Hz}, 1 \mathrm{H}), 5.11(\mathrm{td}, J=5.0,2.2 \mathrm{~Hz}, 1 \mathrm{H}), 4.04(\mathrm{~d}, J=4.5 \mathrm{~Hz}, 1 \mathrm{H}), 1.59(\mathrm{ddt}, J=12.9,7.7,6.5 \mathrm{~Hz}, 1 \mathrm{H}), 1.03(\mathrm{dt}, J=$ $14.8,5.8 \mathrm{~Hz}, 1 \mathrm{H}), 0.89(\mathrm{dtd}, J=14.3,7.1,6.4,2.3 \mathrm{~Hz}, 1 \mathrm{H}), 0.82(\mathrm{t}, J=6.3 \mathrm{~Hz}, 7 \mathrm{H}), 13 \mathrm{C}$ NMR $\left(151 \mathrm{MHz}, \mathrm{CDCl}_{3}\right) \delta$ $142.4,142.4,137.3,135.9,133.3,132.8,130.3,129.2,129.0,128.8,128.6,128.3,127.9,125.9,125.7,125.5,125.3$, 125.1, 41.4, 26.2, 25.5, 25.4, 22.5. ${ }_{29} \mathrm{Si} \mathrm{NMR}\left(79 \mathrm{MHz}, \mathrm{CDCl}_{3}\right) \delta-9.5$. MALDI $\mathrm{m} / z$ calc for $\mathrm{C}_{27} \mathrm{H}_{28} \mathrm{Si}[\mathrm{M}-\mathrm{H}]_{+}$ 379.1877. Found 379.220.

Racemic Standard for $\mathbf{4 k}$ :

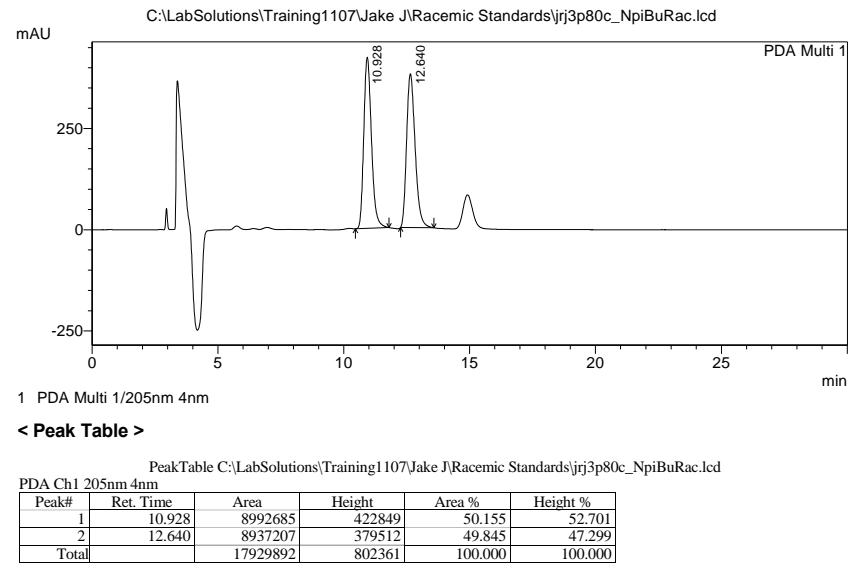

Enantiomerically enriched $(S, S)$ 4k using $S$-TCPTTL:

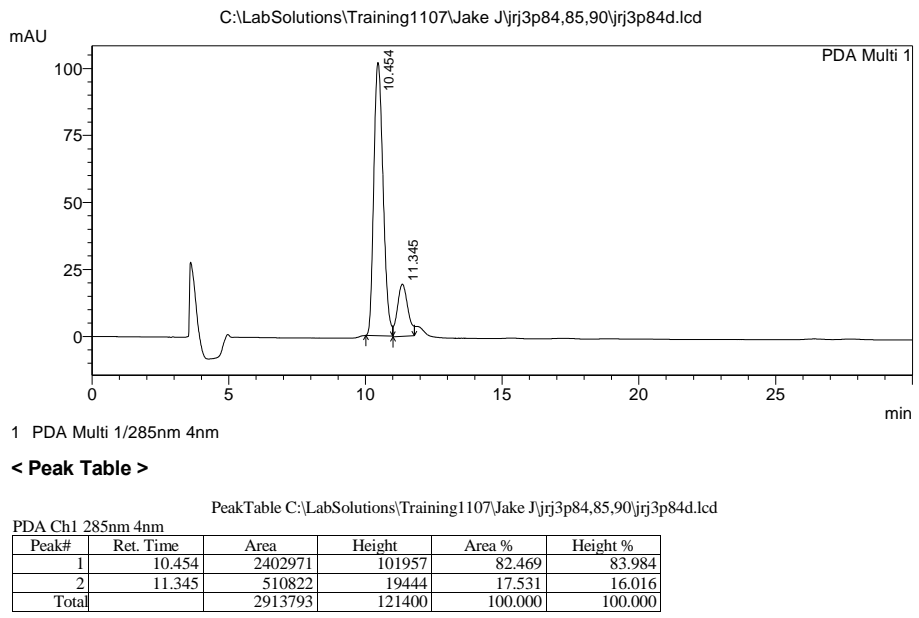




\section{(S)-benzhydryl(isobutyl)(phenyl)silane (4l)}

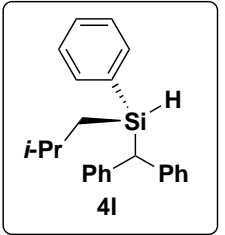

Synthesized using method $\mathrm{F}$ with diazo compound 2a $(0.2 \mathrm{mmol}, 38.8 \mathrm{mg})$ and isobutylphenylsilane $(164.3 \mathrm{mg})$ to give a white solid in $45 \%$ yield $(0.090 \mathrm{mmol}, 29.7$ $\mathrm{mg}$ ). Enantiomeric ratio was determined by HLPC after hydrolysis with a Daicel CHIRALPAK ® OD-H column (1\% IPA/ hexanes), $1.0 \mathrm{~mL} / \mathrm{min}$. tR $(4 \mathbf{l a})=5.6 \mathrm{~min}$, tR $(4 \mathrm{lb})=6.2 \mathrm{~min}, 86: 14 \mathrm{er}(\mathrm{Si}-\mathrm{OH}$ product $)$. Absolute configuration was assigned to be $(S$, $S$ ) based on analogy to $\mathbf{5 a}$.

1H NMR (400 MHz, $\left.\mathrm{CDCl}_{3}\right) \delta 7.40-7.31(\mathrm{~m}, 3 \mathrm{H}), 7.29(\mathrm{~d}, J=5.5 \mathrm{~Hz}, 6 \mathrm{H}), 7.25-7.15(\mathrm{~m}, 5 \mathrm{H}), 7.12(\mathrm{t}, J=6.8 \mathrm{~Hz}$, $1 \mathrm{H}), 4.74(\mathrm{~h}, J=2.2 \mathrm{~Hz}, 1 \mathrm{H}), 3.86(\mathrm{~d}, J=3.9 \mathrm{~Hz}, 1 \mathrm{H}), 1.68(\mathrm{dh}, J=12.5,6.3 \mathrm{~Hz}, 1 \mathrm{H}), 0.93(\mathrm{~d}, J=5.9 \mathrm{~Hz}, 1 \mathrm{H}), 0.89$ $(\mathrm{d}, J=6.6 \mathrm{~Hz}, 6 \mathrm{H}), 0.80(\mathrm{dd}, J=14.7,7.7 \mathrm{~Hz}, 1 \mathrm{H}) .13 \mathrm{C} \mathrm{NMR}\left(101 \mathrm{MHz}, \mathrm{CDCl}_{3}\right) \delta 142.4,142.2,135.4,134.3,129.5$, 129.1, 129.0, 128.6, 128.4, 127.8, 125.6, 125.4, 42.8, 26.1, 25.5, 25.2, 21.7. ${ }_{29} \mathrm{Si} \mathrm{NMR}\left(79 \mathrm{MHz}, \mathrm{CDCl}_{3}\right) \delta$-8.7. Did not ionize using ESI-MS or MALDI-TOF.

\section{Racemic Standard for 4l:}

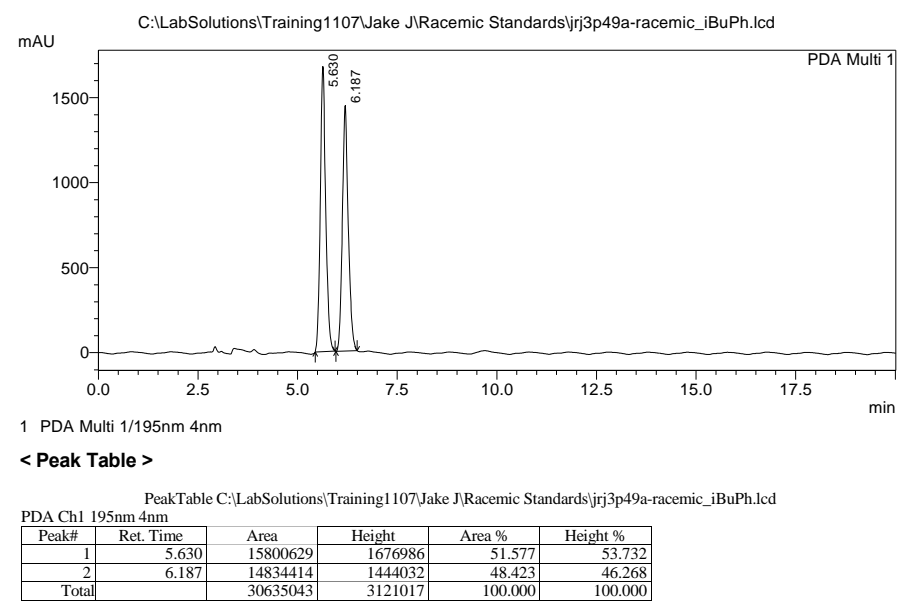

Enantiomerically enriched $(S)$ 4l using $S$-TCPTTL:

$<$ Chromatogram>

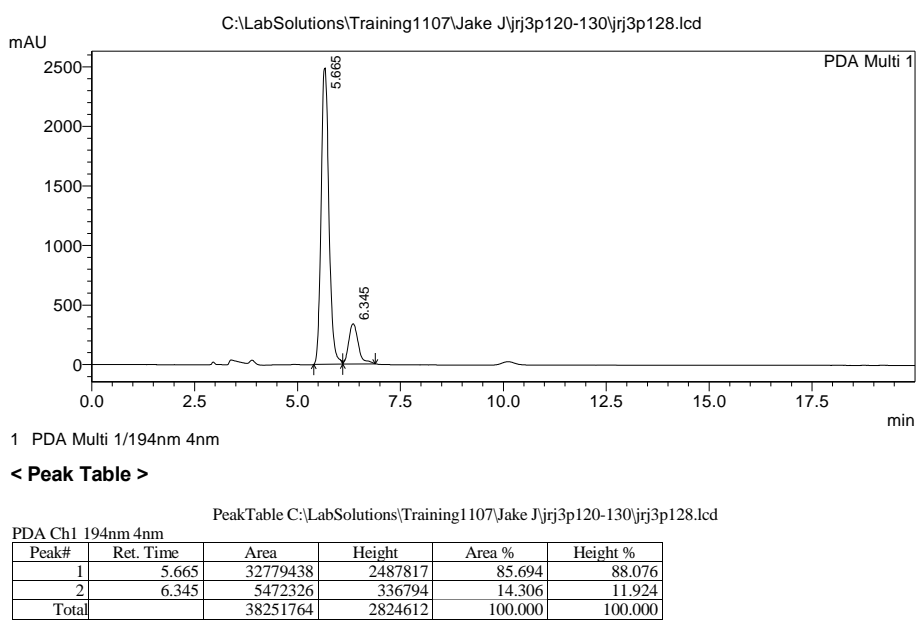




\section{(S)-benzhydryl(neopentyl)(phenyl)silane (4m)}

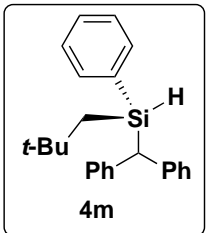

Synthesized using method $\mathrm{F}$ with diazo compound $\mathbf{2 a}(0.2 \mathrm{mmol}, 42.0 \mathrm{mg})$ to give a white solid in $60 \%$ yield $(0.120 \mathrm{mmol}, 41.3 \mathrm{mg})$. Enantiomeric ratio was determined by HLPC after hydrolysis with a Daicel CHIRALPAK $®$ AD-H column (1\% IPA/ heptane), 1.0 $\mathrm{mL} / \mathrm{min}$. tR $(\mathbf{4 m a})=10.6 \mathrm{~min}, \mathrm{tR}(\mathbf{4 m b})=13.1 \mathrm{~min}, 50: 50 \mathrm{er}(\mathrm{Si}-\mathrm{OH}$ product $)$. Absolute configuration was assigned to be $(S)$ based on analogy to $\mathbf{5 a}$.

${ }_{1} \mathrm{H}$ NMR $\left(400 \mathrm{MHz}, \mathrm{CDCl}_{3}\right) \delta 7.37-7.30(\mathrm{~m}, 3 \mathrm{H}), 7.30-7.21(\mathrm{~m}, 6 \mathrm{H}), 7.18(\mathrm{t}, J=6.8 \mathrm{~Hz}, 3 \mathrm{H}), 7.15-7.04(\mathrm{~m}, 3 \mathrm{H})$, $4.81(\mathrm{t}, J=4.7 \mathrm{~Hz}, 1 \mathrm{H}), 3.80(\mathrm{~d}, J=4.0 \mathrm{~Hz}, 1 \mathrm{H}), 1.08(\mathrm{dd}, J=14.7,5.4 \mathrm{~Hz}, 1 \mathrm{H}), 0.93(\mathrm{~s}, 1 \mathrm{H}), 0.89(\mathrm{~s}, 9 \mathrm{H}) .13 \mathrm{C} \mathrm{NMR}$ $\left(101 \mathrm{MHz}, \mathrm{CDCl}_{3}\right) \delta 142.4,142.2,135.4,135.3,129.4,129.3,128.9,128.6,128.3,127.7,125.6,125.3,43.3,32.5$, 30.6, 27.45. $29 \mathrm{Si} \mathrm{NMR}\left(79 \mathrm{MHz}, \mathrm{CDCl}_{3}\right) \delta$-11.1. Did not ionize using ESI, MALDI or APCI.

Racemic Standard for $\mathbf{4 m}$ :

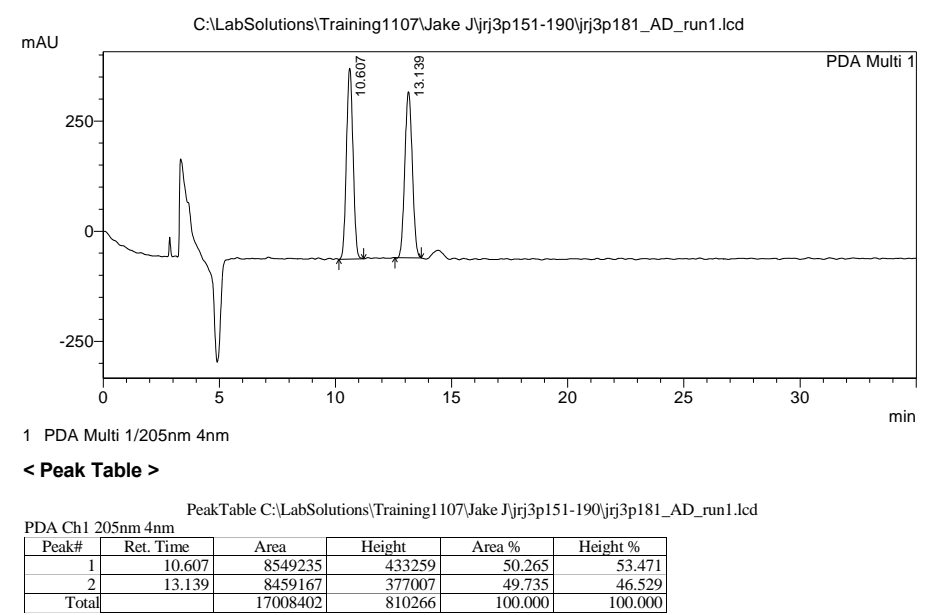

Enantiomerically enriched $(S) \mathbf{4 m}$ using $S$-TCPTTL:

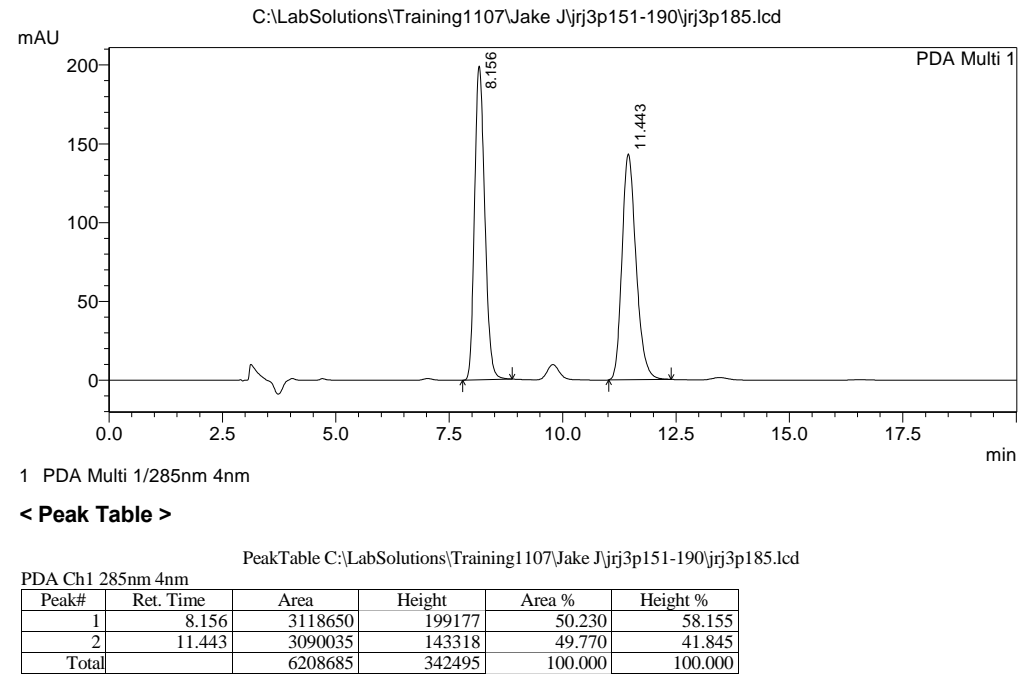




\section{(S)-benzhydryl(cyclohexyl)(phenyl)silane (4n)}

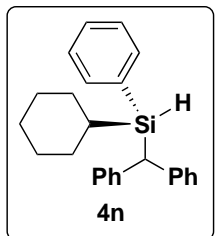

Synthesized using method F with diazo compound $\mathbf{2 a}(0.2 \mathrm{mmol}, 38.8 \mathrm{mg})$ to give a white solid in $77 \%$ yield $(0.158 \mathrm{mmol}, 54.9 \mathrm{mg})$. Enantiomeric ratio was determined by HLPC after hydrolysis with a Daicel CHIRALPAK ® AD-H column (2\% IPA/ hexanes), 1.0 $\mathrm{mL} / \mathrm{min}$. tR $(\mathbf{4 n a})=37.7 \mathrm{~min}, \mathrm{tR}(\mathbf{4 n b})=42.6 \mathrm{~min}, 70: 30$ er $(\mathrm{Si}-\mathrm{OH}$ product $)$. Absolute configuration was assigned to be $(S)$ based on analogy to $\mathbf{5 a}$.

1H NMR (600 MHz, $\left.\mathrm{CDCl}_{3}\right) \delta 7.34-7.27(\mathrm{~m}, 6 \mathrm{H}), 7.27-7.20(\mathrm{~m}, 4 \mathrm{H}), 7.19-7.10(\mathrm{~m}, 5 \mathrm{H}), 7.07-7.01(\mathrm{~m}, 1 \mathrm{H})$, $4.48(\mathrm{dd}, J=4.5,2.9 \mathrm{~Hz}, 1 \mathrm{H}), 3.96(\mathrm{~d}, J=4.4 \mathrm{~Hz}, 1 \mathrm{H}), 1.72-1.62(\mathrm{~m}, 1 \mathrm{H}), 1.65-1.55(\mathrm{~m}, 4 \mathrm{H}), 1.17-1.01(\mathrm{~m}, 5 \mathrm{H})$, $1.00-0.90(\mathrm{~m}, 1 \mathrm{H}) . \mathrm{NMR}\left(151 \mathrm{MHz}, \mathrm{CDCl}_{3}\right) \delta 142.6,142.3,135.8,133.2,129.4,129.2,129.0,128.6,128.4,127.7$, 125.7, 125.4, 40.5, 28.5, 28.1, 27.8, 26.8, 22.9. $29 \mathrm{Si}$ NMR (79 MHz, $\left.\mathrm{CDCl}_{3}\right) \delta$-2.8. MALDI $m / z$ calc for $\mathrm{C}_{25} \mathrm{H}_{28} \mathrm{Si}$ [M $+\mathrm{Na}]+379.185$. Found 379.140 .

Racemic Standard for $\mathbf{4 n}$ :

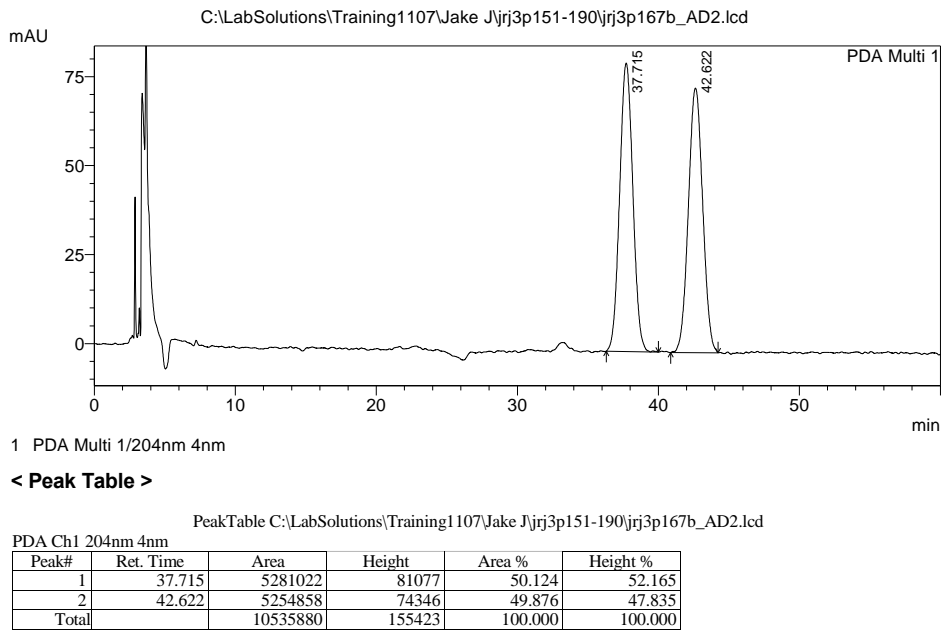

Enantiomerically enriched $(S, S)$ 4n using $S$-TCPTTL:

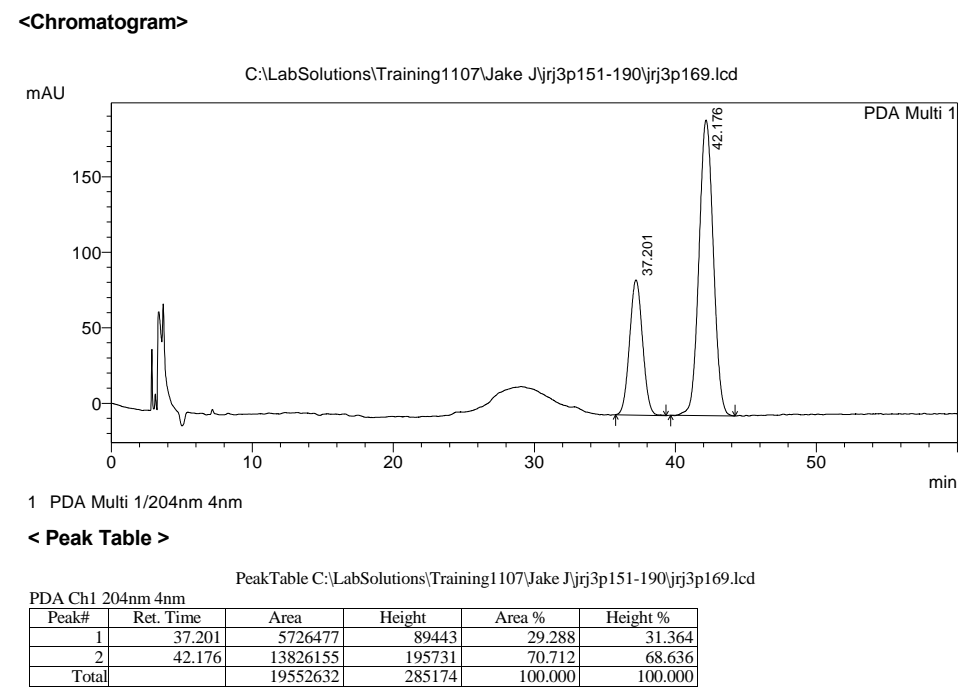




\section{(S)-3-benzhydryl-1,1,1-triethyl-3-phenyldisiloxane (40)}

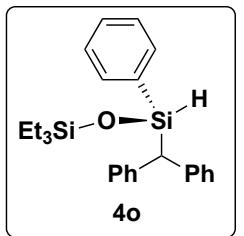

Synthesized using method $F$ with diazo compound $\mathbf{2 a}(0.2 \mathrm{mmol}, 38.8 \mathrm{mg})$ to give a white solid in $60 \%$ yield $(0.120 \mathrm{mmol}, 41.3 \mathrm{mg})$. Enantiomeric ratio was determined by HLPC after hydrolysis with a Daicel CHIRALPAK ® AD-H column (2\% IPA/ hexanes), $1.0 \mathrm{~mL} / \mathrm{min} . \mathrm{tR}_{\mathrm{R}}(\mathbf{4 0 a})=9.5 \mathrm{~min}, \mathrm{tR}(\mathbf{4 o b})=10.6 \mathrm{~min}, 61: 39 \mathrm{er}(\mathrm{Si}-\mathrm{OH}$ product $)$. Absolute configuration was assigned to be $(S)$ based on analogy to $\mathbf{5 a}$.

${ }_{1} \mathrm{H}$ NMR $\left(600 \mathrm{MHz}, \mathrm{CDCl}_{3}\right) \delta 7.37-7.31(\mathrm{~m}, 1 \mathrm{H}), 7.31-7.25(\mathrm{~m}, 8 \mathrm{H}), 7.25-7.20(\mathrm{~m}, 3 \mathrm{H}), 7.20-7.09(\mathrm{~m}, 3 \mathrm{H})$, $5.30(\mathrm{~d}, J=2.6 \mathrm{~Hz}, 1 \mathrm{H}), 3.80(\mathrm{~d}, J=2.6 \mathrm{~Hz}, 1 \mathrm{H}), 0.80(\mathrm{t}, J=8.0 \mathrm{~Hz}, 6 \mathrm{H}), 0.41(\mathrm{q}, J=7.7 \mathrm{~Hz}, 6 \mathrm{H}) .13 \mathrm{C} \mathrm{NMR}(101$ $\left.\mathrm{MHz}_{2} \mathrm{CDCl}_{3}\right) \delta 141.1,141.0,135.5,134.1,130.0,129.5,129.1,128.5,128.5,127.7,125.7,125.6,44.4,6.7,6.0 .29 \mathrm{Si}$ NMR (79 MHz, $\left.\mathrm{CDCl}_{3}\right) \delta-14.5,-18.4 /$ Did not ionize using ESI, MALDI or APCI.

Racemic Standard for 4o:

<Chromatogram>

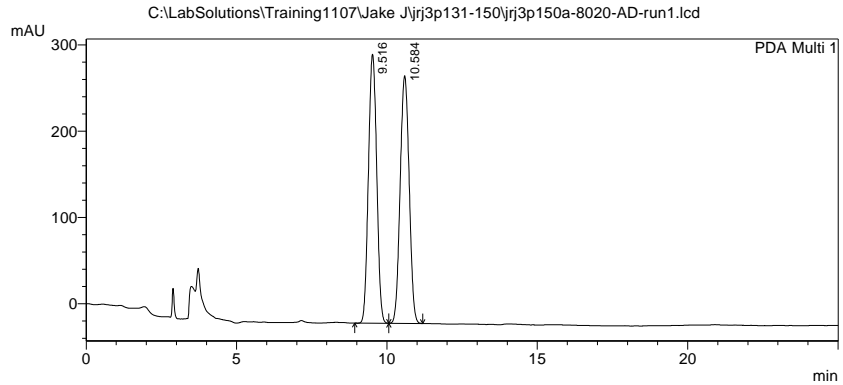

1 PDA Multi $1 / 204 \mathrm{~nm} 4 \mathrm{~nm}$

<Peak Table >

PeakTable C:LabSolutionsiTraining1 107Uake Jjirj3p131-150jiji3p150a-8020-AD-run1.lcd

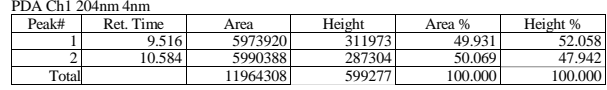

Enantiomerically enriched $(S, S)$ 4o using $S$-TCPTTL:

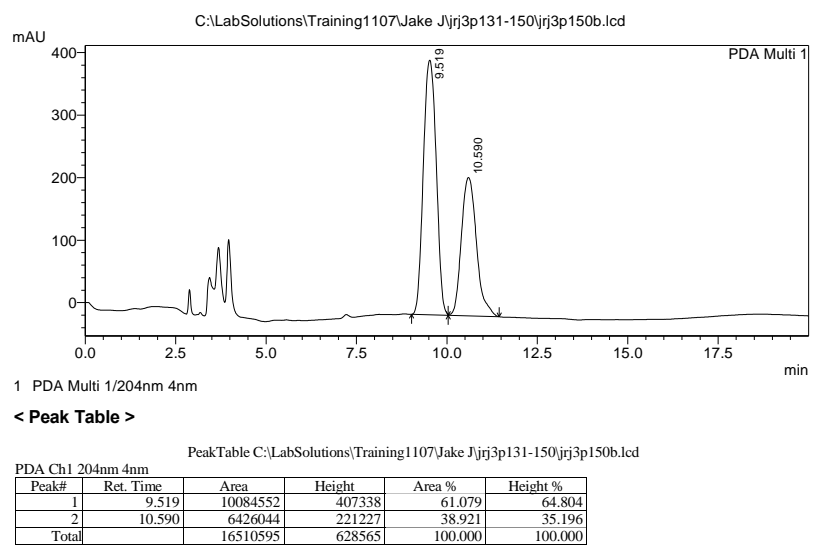




\section{(S)-methyl(phenyl)((S)-phenyl(o-tolyl)methyl)silane (5a):}

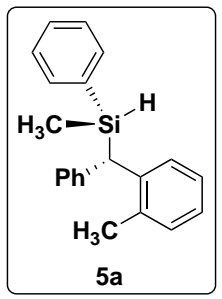

Synthesized using method $\mathrm{G}$ with Diazo compound 3a $(5.1 \mathrm{mmol}, 1.00 \mathrm{~g})$ to give a clear oil in $89 \%$ yield ( $93: 7 \mathrm{dr}, 1.34 \mathrm{~g}, 4.5 \mathrm{mmol})$. Enantiomeric ratio was determined by HLPC after hydrolysis with a Daicel CHIRALPAK ® AD-H column (1\% IPA/ heptane), $1.0 \mathrm{~mL} / \mathrm{min}$.

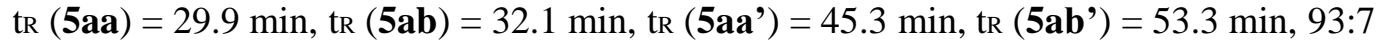
er (Si-OH product, 5ab:5ab'). Absolute configuration was assigned to be $(S, S)$ based on analogy to $\mathbf{5 e}$ and $\mathbf{1 1 .}$

$\left.{ }_{1} \mathrm{H} \mathrm{NMR}\left(600 \mathrm{MHz}, \mathrm{CDCl}_{3}\right)\right) \delta 7.45-7.04(\mathrm{~m}, 14 \mathrm{H}), 4.72(\mathrm{q}, J=3.6 \mathrm{~Hz}, 3 \mathrm{H}), 3.96(\mathrm{~d}, J=4.0 \mathrm{~Hz}, 1 \mathrm{H}), 2.21(\mathrm{~s}, 3 \mathrm{H})$, $0.33(\mathrm{~d}, J=3.6 \mathrm{~Hz}, 3 \mathrm{H}) .{ }_{13} \mathrm{C}$ NMR $\left(151 \mathrm{MHz}, \mathrm{CDCl}_{3}\right) \delta 142.0,140.5,137.0,135.1,135.0,139.0,129.7,129.6,128.9$, 128.3, 127.8, 126.1 , 125.9, 125.2, 38.7, 20.3, -5.6. $\left.29 \mathrm{Si} \mathrm{NMR} \mathrm{(119} \mathrm{MHz,} \mathrm{CDCl}_{3}\right) \delta-10.2$. MALDI $m / z$ calc for C21 $\mathrm{H}_{22} \mathrm{Si}$ $[\mathrm{M}+\mathrm{H}]+303.156$. Found 303.154 .

Racemic Standard for 5a:

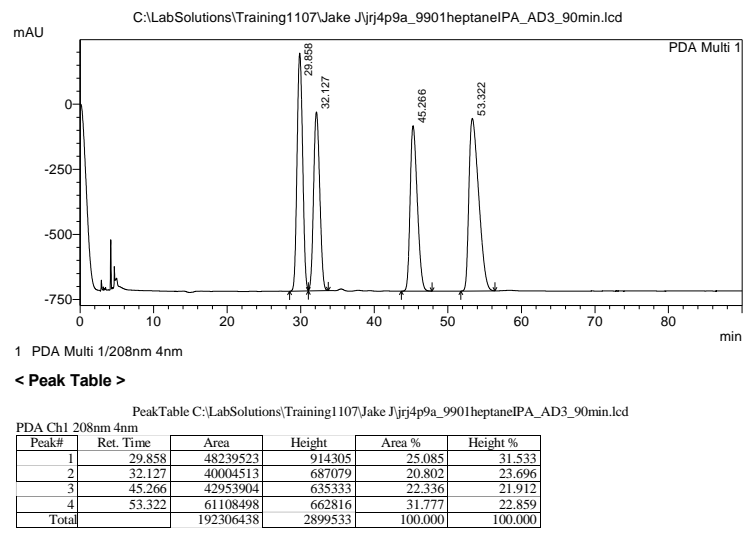

Enantiomerically enriched $(S, S)$ 5a using $S$-TCPTTL:

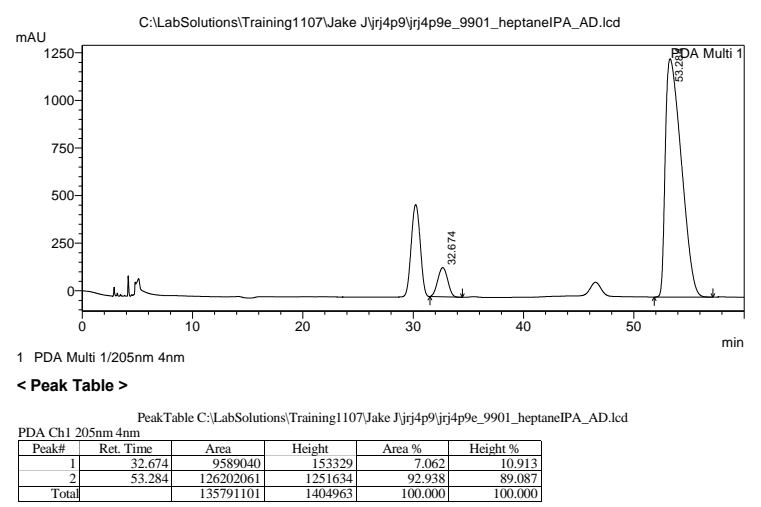


Enantiomerically enriched $(R, R)$ 5a using $R$-TCPTTL:

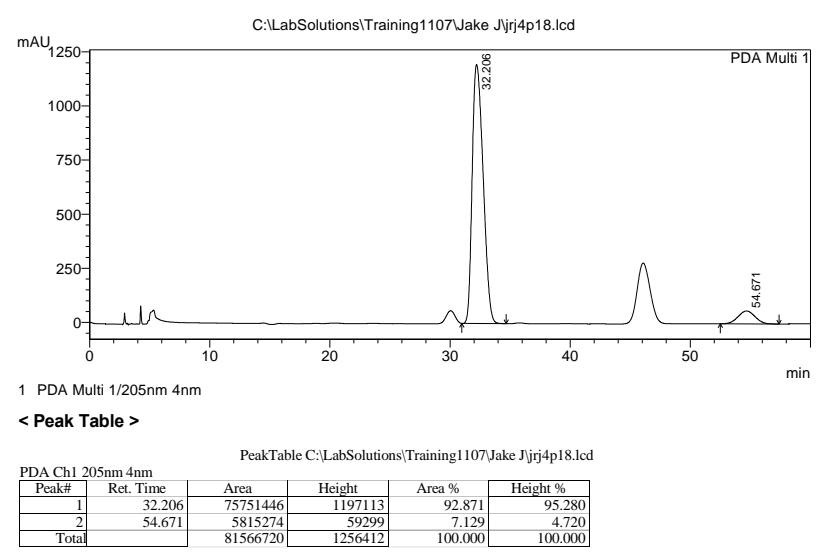




\section{$(S)-((S)-(2-m e t h o x y p h e n y l)($ phenyl)methyl)(methyl)(phenyl)silane (5b):}

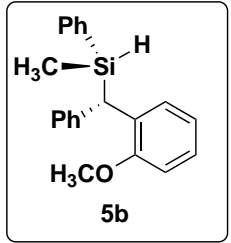

Synthesized using method $\mathrm{F}$ with diazo compound $\mathbf{3 b}(0.2 \mathrm{mmol}, 48.9 \mathrm{mg})$ to give a clear solid in $91 \%$ yield $(0.182 \mathrm{mmol}, 58.0 \mathrm{mg}, 90: 10 \mathrm{dr})$. Enantiomeric ratio was determined by HLPC after hydrolysis with a Daicel CHIRALPAK ® AD-H column (2\% IPA/ hexanes $), 1.0 \mathrm{~mL} / \mathrm{min}$. $\mathrm{t}_{\mathrm{R}}(\mathbf{5 b a})=26.6 \mathrm{~min}, \mathrm{t}_{\mathrm{R}}(\mathbf{5 b b})=29.0 \mathrm{~min}, \mathrm{tR}_{\mathrm{R}}(\mathbf{5} \mathbf{b} \mathbf{b})=36.8$ min, tR $(\mathbf{5 b b}$ ') $=42.9$ min, 95:5 er (Si-OH product, 5ba':5ba) Absolute configuration was assigned to be $(S, S)$ based on analogy to $\mathbf{5 a}$.

1H NMR (600 MHz, CDCl $) \delta 7.33(\mathrm{~d}, J=7.6 \mathrm{~Hz}, 2 \mathrm{H}), 7.30(\mathrm{~d}, J=7.2 \mathrm{~Hz}, 1 \mathrm{H}), 7.24(\mathrm{dt}, J=11.6,5.4 \mathrm{~Hz}, 3 \mathrm{H}), 7.19$ $-7.11(\mathrm{~m}, 5 \mathrm{H}), 7.09-7.05(\mathrm{~m}, 1 \mathrm{H}), 6.88(\mathrm{t}, J=7.4 \mathrm{~Hz}, 1 \mathrm{H}), 6.82(\mathrm{~d}, J=8.2 \mathrm{~Hz}, 1 \mathrm{H}), 4.70-4.66(\mathrm{~m}, 1 \mathrm{H}), 4.26(\mathrm{~d}, J$ $=3.5 \mathrm{~Hz}, 1 \mathrm{H}), 3.72(\mathrm{~s}, 3 \mathrm{H}), 0.25(\mathrm{~d}, J=3.4 \mathrm{~Hz}, 3 \mathrm{H}) .13 \mathrm{C} \mathrm{NMR}\left(151 \mathrm{MHz}, \mathrm{CDCl}_{3}\right) \delta 156.9,142.5,136.0,134.9,131.0$, $130.2,129.3,129.2,128.3,127.7,126.8,125.2,120.7,110.6,55.3,35.3,-5.7 .29 \mathrm{Si}$ NMR $\left(76 \mathrm{MHz}, \mathrm{CDCl}_{3}\right) \delta-12.5$. ESI-MS $m / z$ calc for $\mathrm{C}_{21} \mathrm{H}_{22} \mathrm{O} 2 \mathrm{Si}(\mathrm{Si}-\mathrm{OH})[\mathrm{M}-\mathrm{H}]-333.1316$. Found: 333.1304 .

Racemic Standard for $\mathbf{5 b}$ :

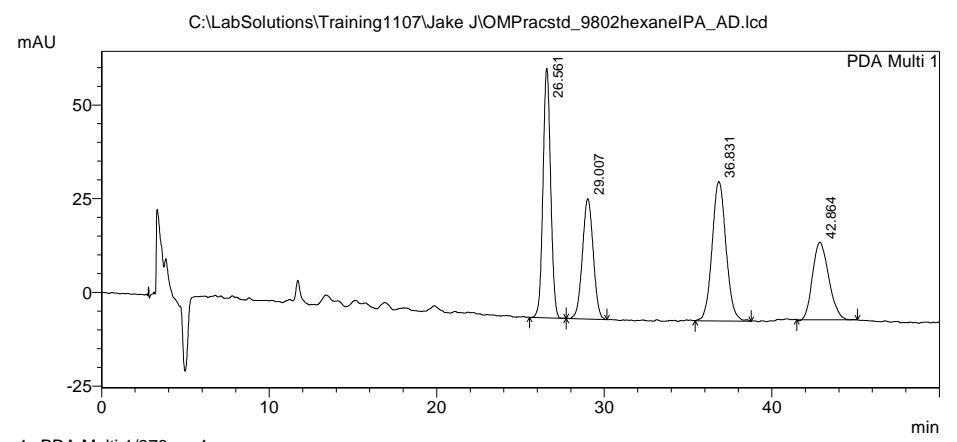

1 PDA Multi $1 / 273 \mathrm{~nm} 4 \mathrm{~nm}$

< Peak Table >

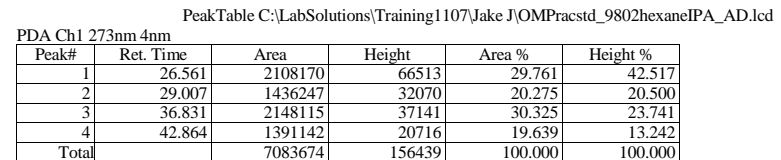

Enantiomerically enriched $(S, S)$ 5b using $S$-TCPTTL:

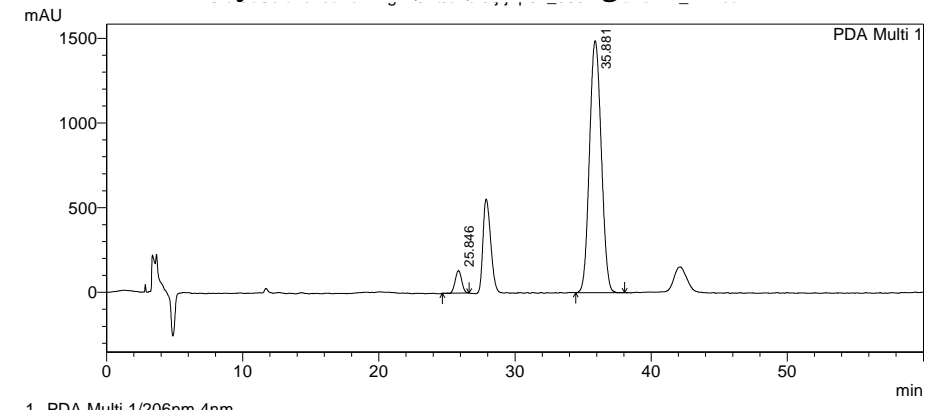

1 PDA Multi $1 / 206 \mathrm{~nm} 4 \mathrm{~nm}$

< Peak Table >

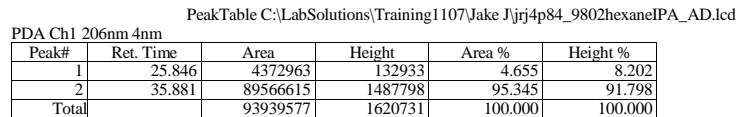


Enantiomerically enriched $(R, R) \mathbf{5 b}$ using $R$-TCPTTL:

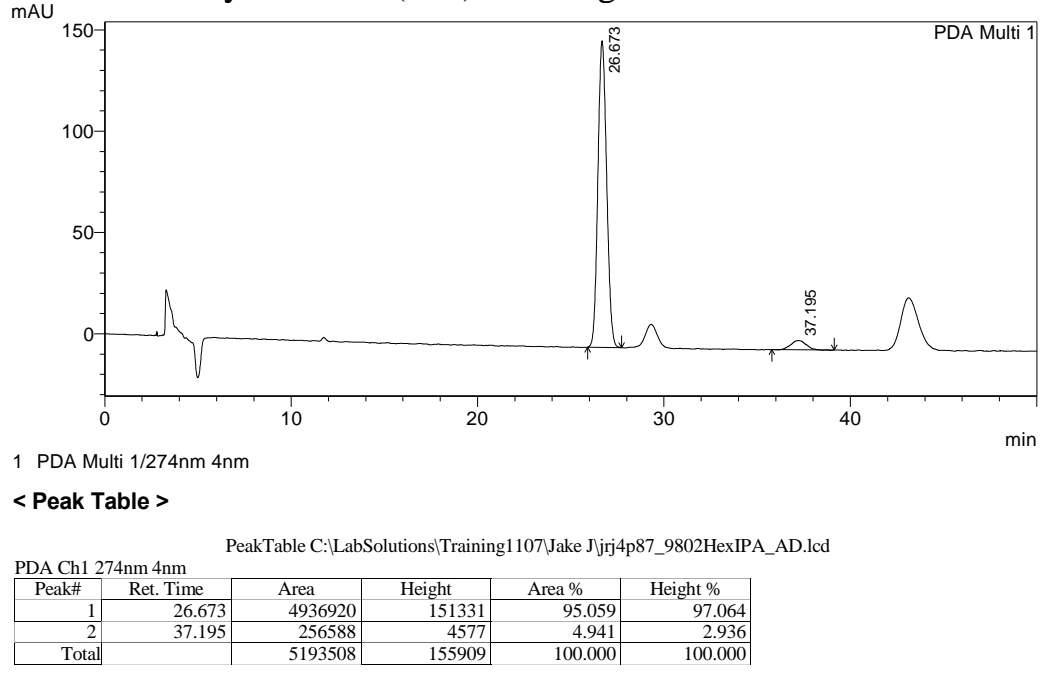




\section{(S)-methyl(phenyl)((S)-phenyl(2-(trifluoromethyl)phenyl)methyl)silane (5c):}

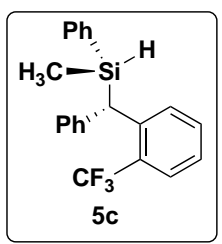

Synthesized using method $\mathrm{F}$ with diazo compound $\mathbf{3 c}(0.2 \mathrm{mmol}, 42.0 \mathrm{mg})$ to give a white solid in $93 \%$ yield $\left(0.186 \mathrm{mmol}, 66.3 \mathrm{mg}, 98: 2 \mathrm{dr}\right.$ (determined using ${ }_{19} \mathrm{~F}$ NMR). Enantiomeric ratio was determined by HLPC after hydrolysis with a Daicel CHIRALPAK ${ }^{\circledR} \mathrm{AD}-\mathrm{H}$ column $(2 \% \mathrm{IPA} /$ hexanes $), 1.0 \mathrm{~mL} / \mathrm{min} . \mathrm{tR}(\mathbf{5} \mathbf{c a})=14.7 \mathrm{~min}, \mathrm{tR}(\mathbf{5 c b})=15.7$ $\min , t_{R}\left(\mathbf{5} \mathbf{c b}^{\prime}\right)=23.4$ min, tR $\left(\mathbf{5} \mathbf{c a}^{\prime}\right)=26.6 \mathrm{~min}, 93: 7$ er $(\mathrm{Si}-\mathrm{OH}$ product, $\mathbf{5} \mathbf{c b} ' \mathbf{5} \mathbf{c b})$ Absolute configuration was assigned to be $(S, S)$ based on analogy to $\mathbf{5 a}$.

1H NMR (600 MHz, $\left.\mathrm{CDCl}_{3}\right) \delta 7.63(\mathrm{dd}, J=12.3,7.9 \mathrm{~Hz}, 2 \mathrm{H}), 7.50(\mathrm{t}, J=7.6 \mathrm{~Hz}, 1 \mathrm{H}), 7.34(\mathrm{~d}, J=7.1 \mathrm{~Hz}, 3 \mathrm{H}), 7.29$ $-7.22(\mathrm{~m}, 4 \mathrm{H}), 7.21(\mathrm{~d}, J=7.5 \mathrm{~Hz}, 2 \mathrm{H}), 7.17(\mathrm{t}, J=7.6 \mathrm{~Hz}, 2 \mathrm{H}), 7.10(\mathrm{t}, J=7.2 \mathrm{~Hz}, 1 \mathrm{H}), 4.76(\mathrm{p}, J=4.0 \mathrm{~Hz}, 1 \mathrm{H})$, $4.22(\mathrm{~d}, J=4.7 \mathrm{~Hz}, 1 \mathrm{H}), 0.20(\mathrm{~d}, J=3.7 \mathrm{~Hz}, 4 \mathrm{H}) .13 \mathrm{C} \mathrm{NMR}\left(151 \mathrm{MHz}, \mathrm{CDCl}_{3}\right) \delta 141.7,141.7,141.4,134.9,134.3$, $131.9,131.4,129.8,128.8,128.5,128.5\left(\mathrm{q}, J_{\mathrm{CCCF}}=7.0 \mathrm{~Hz}\right), 128.3,128.1\left(\mathrm{q}, J_{\mathrm{CCF}}=36.0 \mathrm{~Hz}\right), 127.9,126.4\left(\mathrm{q}, J_{\mathrm{CCF}}=\right.$ $5.9 \mathrm{~Hz}, 125.8,125.7,124.8$ (q, J $C F=274 \mathrm{~Hz}), 37.80,-5.80 .19 \mathrm{~F} \mathrm{NMR}\left(376 \mathrm{MHz}, \mathrm{CDCl}_{3}\right) \delta-58.2 .{ }_{29} \mathrm{Si} \mathrm{NMR}(76 \mathrm{MHz}$, $\left.\mathrm{CDCl}_{3}\right) \delta$-9.2. MALDI $m / z$ calc for $\mathrm{C}_{21} \mathrm{H}_{19} \mathrm{~F}_{3} \mathrm{Si}[\mathrm{M}+\mathrm{Na}]+379.110$. Found 379.100

Racemic Standard for $\mathbf{5 c}$ :

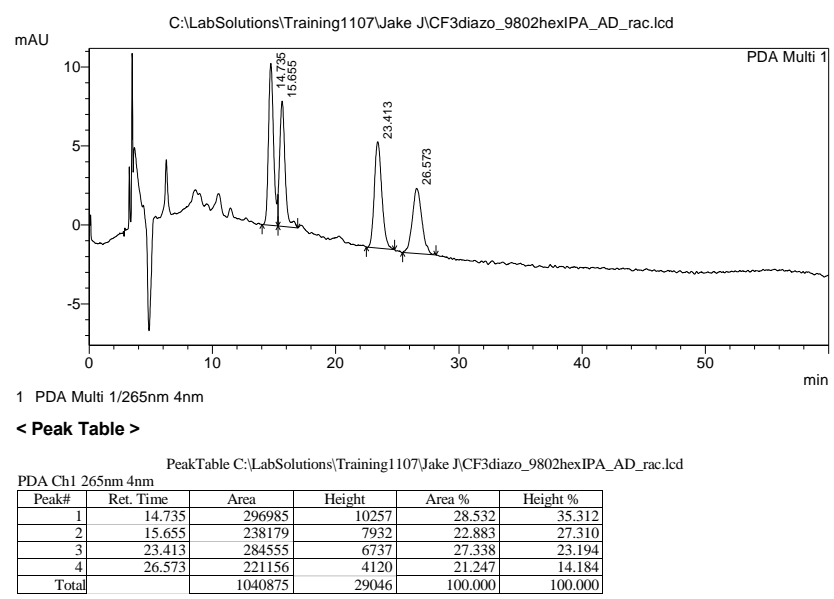

Spectrum index for racemic standard of $\mathbf{5 c}$ :
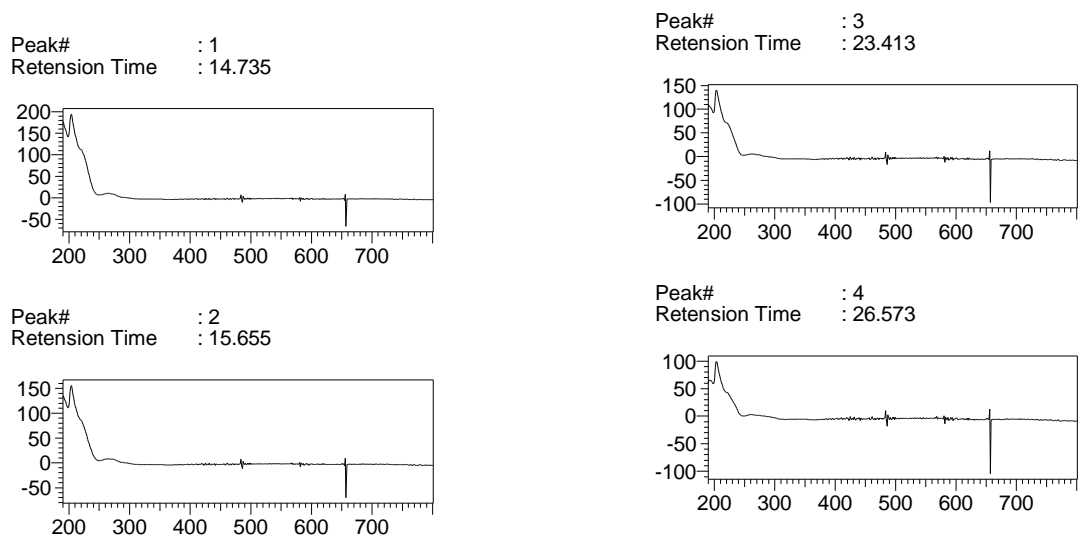
Enantiomerically enriched $(S, S) \mathbf{5 c}$ using $S$-TCPTTL:

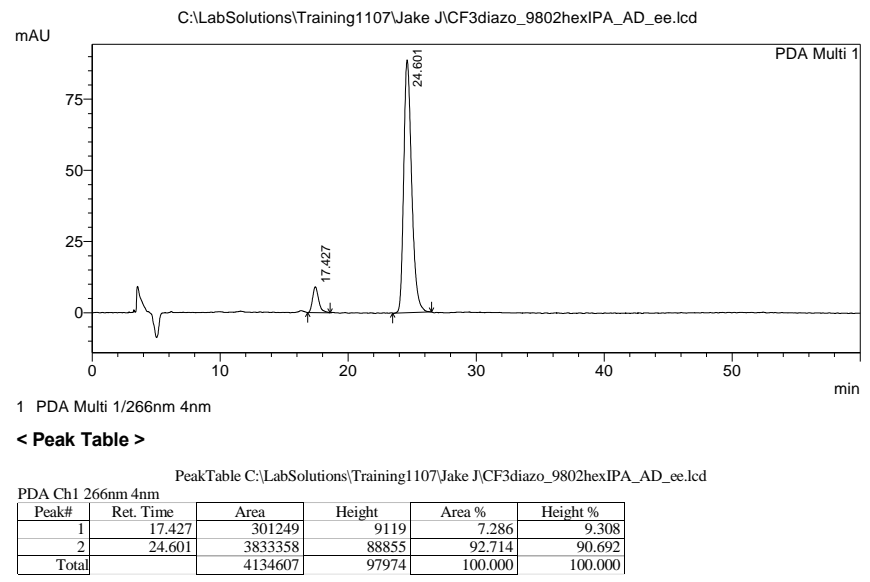

Spectrum index for enantioenriched sample of $\mathbf{5 c}$ :

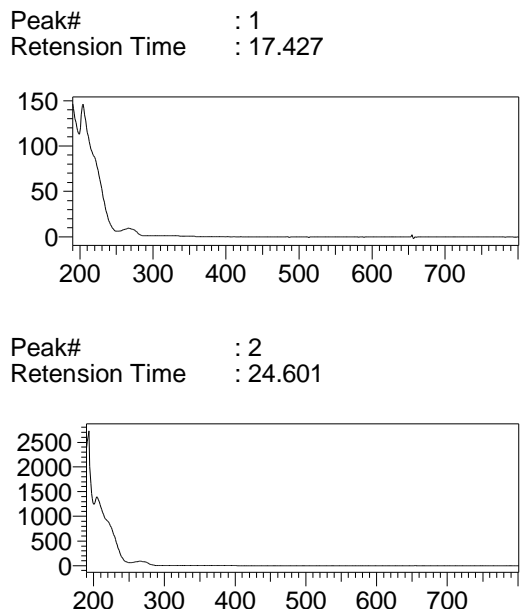

Spectrum indices between racemic standard and enantioenriched are identical, indicating the peaks are identical despite slightly different retention times. 


\section{$(S)-((S)-(2-c h l o r o p h e n y l)(4-m e t h o x y p h e n y l) m e t h y l)(m e t h y l)(p h e n y l) s i l a n e ~(5 d)$}

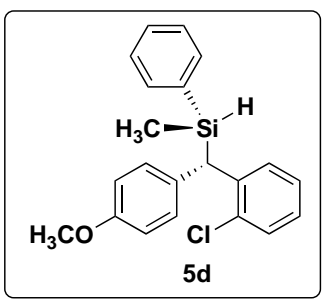

Synthesized using method F with diazo compound $3 \mathbf{e}(0.2 \mathrm{mmol}, 52.0 \mathrm{mg})$ to give a white solid in $98 \%$ yield $(0.196 \mathrm{mmol}, 69.0 \mathrm{mg}, 90: 10 \mathrm{dr}$. Enantiomeric ratio was determined by HLPC after hydrolysis with a Daicel CHIRALPAK ${ }^{\circledR}$ AD-H column $(2 \% \mathrm{IPA} /$ heptane $), 1.0 \mathrm{~mL} / \mathrm{min}$. $\mathrm{tR}(\mathbf{5 d a})=49.1 \mathrm{~min}, \mathrm{tR}_{\mathrm{R}}(\mathbf{5 d b})=52.5 \mathrm{~min}$, $\mathrm{tR}_{\mathrm{R}}\left(\mathbf{5} \mathbf{d a}^{\prime}\right)=55.0 \mathrm{~min}, \mathrm{tR}\left(\mathbf{5} \mathbf{d b} \mathbf{b}^{\prime}\right)=87.0 \mathrm{~min}, 89: 11 \mathrm{er}(\mathrm{Si}-\mathrm{OH}$ product, 5da':5da $)$ Absolute configuration was assigned to be $(S, S)$ based on analogy to $\mathbf{5 a}$.

${ }_{1} \mathrm{H}$ NMR $\left(600 \mathrm{MHz} \mathrm{CDCl}_{3}\right) \delta 7.39(\mathrm{~m}, 5 \mathrm{H}), 7.31(\mathrm{t}, J=7.2 \mathrm{~Hz}, 2 \mathrm{H}), 7.22(\mathrm{t}, J=7.6 \mathrm{~Hz}, 1 \mathrm{H}), 7.16(\mathrm{~d}, J=8.2 \mathrm{~Hz}$, $2 \mathrm{H}), 7.12(\mathrm{t}, J=7.7 \mathrm{~Hz}, 1 \mathrm{H}), 6.78(\mathrm{~d}, J=8.4 \mathrm{~Hz}, 2 \mathrm{H}), 4.82-4.76(\mathrm{~m}, 1 \mathrm{H}), 4.45(\mathrm{~d}, J=4.5 \mathrm{~Hz}, 1 \mathrm{H}), 3.76(\mathrm{~s}, 3 \mathrm{H})$, $0.38(\mathrm{~d}, J=3.4 \mathrm{~Hz}, 3 \mathrm{H}) .13 \mathrm{C}$ NMR $\left(151 \mathrm{MHz}, \mathrm{CDCl}_{3}\right) \delta 157.7,140.7,135.0,134.6,134.1,133.3,130.4,130.3$, 129.9, 129.7, 127.9, 127.0, 126.8, 113.9, 75.9, 53.2, 36.2, -5.2. 29Si NMR (79 MHz, CDCl $) \delta-11.34$. MALDI $m / z$ calc for $\mathrm{C}_{21} \mathrm{H}_{21} \mathrm{ClOSi}[\mathrm{M}-\mathrm{H}]+351.097$. Found 351.083.

Racemic Standard for $\mathbf{5 d}$ :

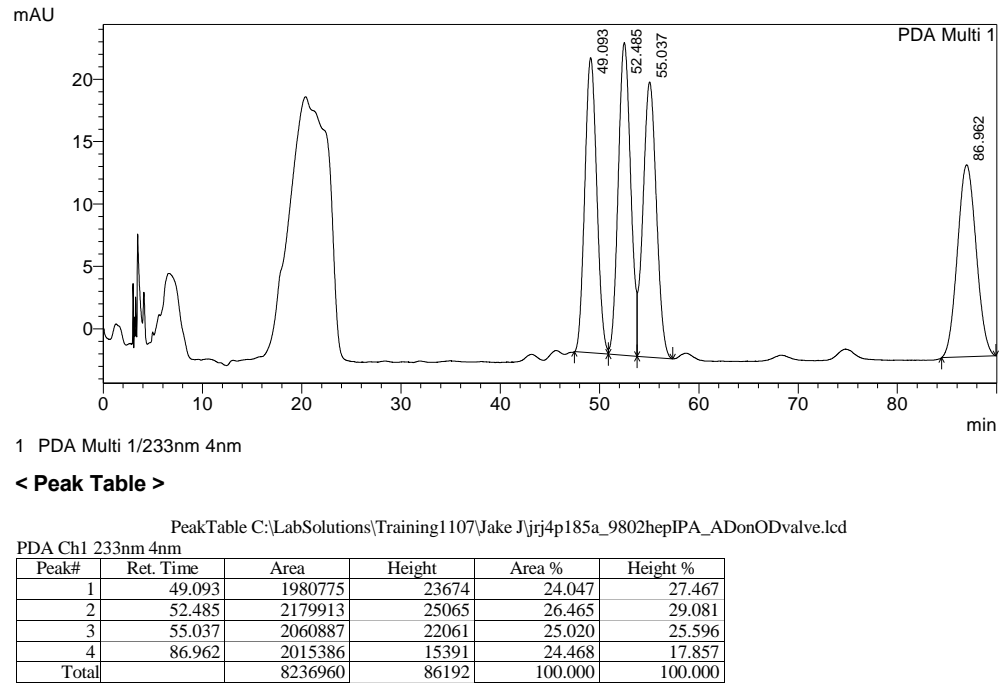

Enantiomerically enriched $(S, S)$ 5d using $S$-TCPTTL:

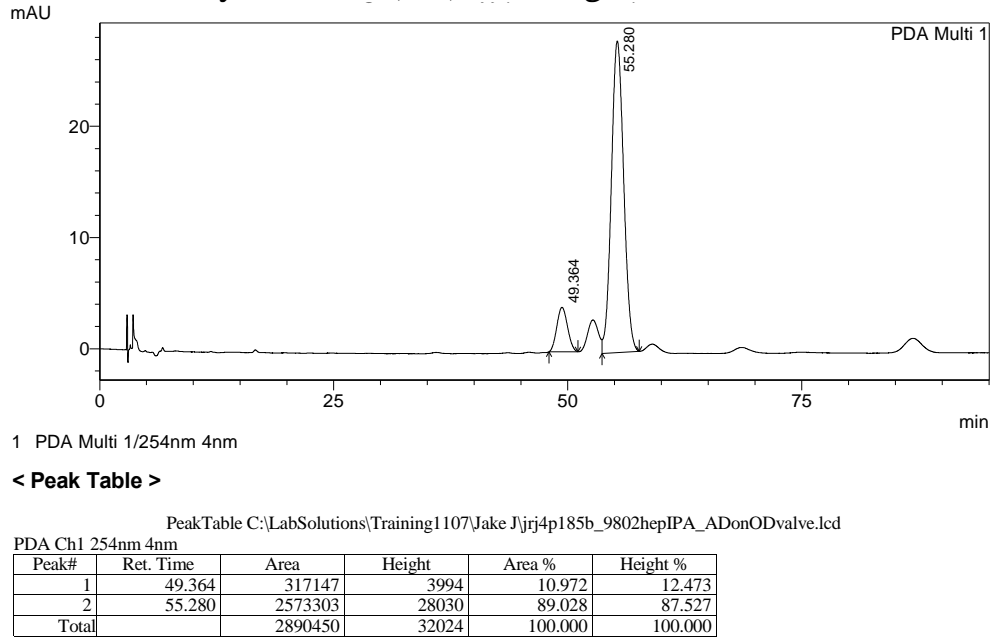


Enantiomerically enriched $(R, R) \mathbf{5 d}$ using $R$-TCPTTL:

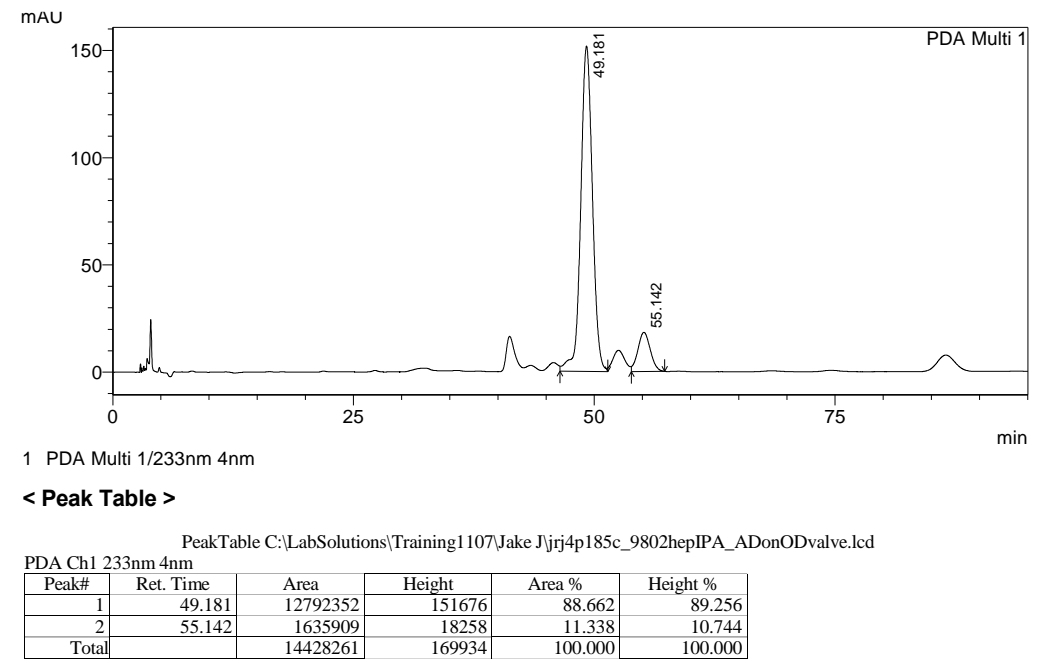




\section{$(S)$-methyl((S)-naphthalen-1-yl(phenyl)methyl)(phenyl)silane (5e):}

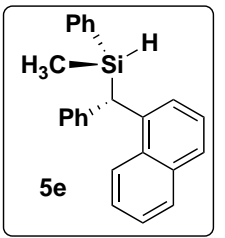

Synthesized using method $\mathrm{F}$ with diazo compound $\mathbf{3 d}(0.2 \mathrm{mmol}, 48.9 \mathrm{mg})$ to give a white solid in $78 \%$ yield $(0.158 \mathrm{mmol}, 52.8 \mathrm{mg}, 85: 15 \mathrm{dr})$. Enantiomeric ratio was determined by HLPC after hydrolysis with a Daicel CHIRALPAK $®$ AD-H column (2\% IPA/ hexanes), $1.0 \mathrm{~mL} / \mathrm{min} . \mathrm{tR}_{\mathrm{R}}(\mathbf{5 e a})=36.0 \mathrm{~min}, \mathrm{tR}(\mathbf{5 e a})=46.3 \mathrm{~min}, \mathrm{tR}(\mathbf{5 e b})=50.8 \mathrm{~min}, \mathrm{tR}_{\mathrm{R}}(\mathbf{5 e b})=$ $56.5 \mathrm{~min}, 61: 39$ er (Si-OH product, 5ea:5ea') Absolute configuration was assigned to be $(S, S)$ based on analogy to $\mathbf{5 a}$.

1H NMR (600 MHz, CDCl $) \delta 8.12-8.05(\mathrm{~m}, 1 \mathrm{H}), 7.82-7.78(\mathrm{~m}, 1 \mathrm{H}), 7.70(\mathrm{~d}, J=8.3 \mathrm{~Hz}, 1 \mathrm{H}), 7.62(\mathrm{~d}, J=7.1 \mathrm{~Hz}$, $3 \mathrm{H}), 7.46-7.36(\mathrm{~m}, 3 \mathrm{H}), 7.35-7.29(\mathrm{~m}, 2 \mathrm{H}), 7.27-7.16(\mathrm{~m}, 1 \mathrm{H}), 7.11(\mathrm{~m}, 3 \mathrm{H}), 7.08-7.00(\mathrm{~m}, 1 \mathrm{H}), 4.83(\mathrm{p}, J=$ $3.2 \mathrm{~Hz}, 1 \mathrm{H}), 4.56(\mathrm{~d}, J=4.2 \mathrm{~Hz}, 1 \mathrm{H}), 0.33(\mathrm{~d}, J=3.6 \mathrm{~Hz}, 3 \mathrm{H}) .13 \mathrm{C}$ NMR $\left(151 \mathrm{MHz}, \mathrm{CDCl}_{3}\right) \delta 142.3,138.2,135.1$, $134.5,132.8,129.7,129.0,128.7,128.5,128.4,127.8,127.4,126.9,126.0,125.6,125.4,125.3,124.2,38.1,-5.4 .29 \mathrm{Si}$ NMR $\left(119 \mathrm{MHz}, \mathrm{CDCl}_{3}\right) \delta-10.0$. Did not ionize using ESI, MALDI or APCI.

Racemic Standard for 5e:

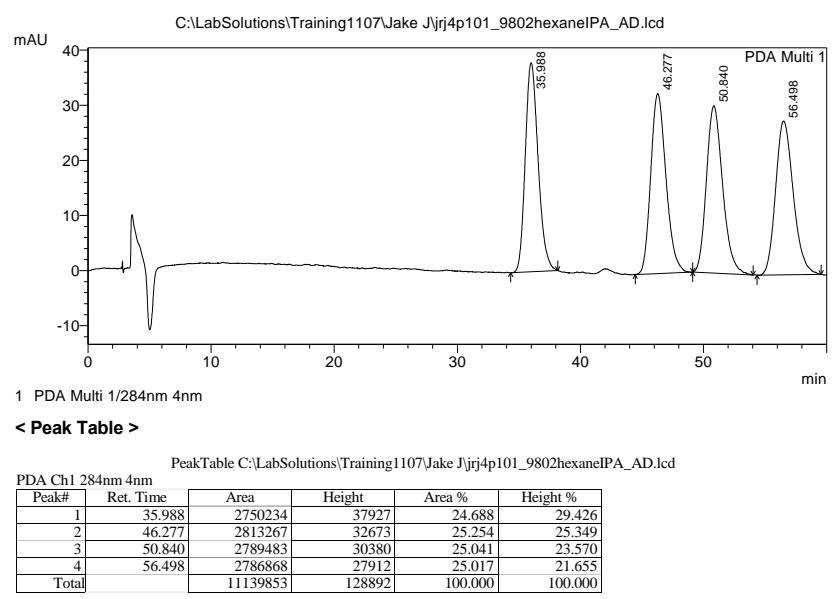

Enantiomerically enriched $(S, S)$ 5e using $S$-TCPTTL:

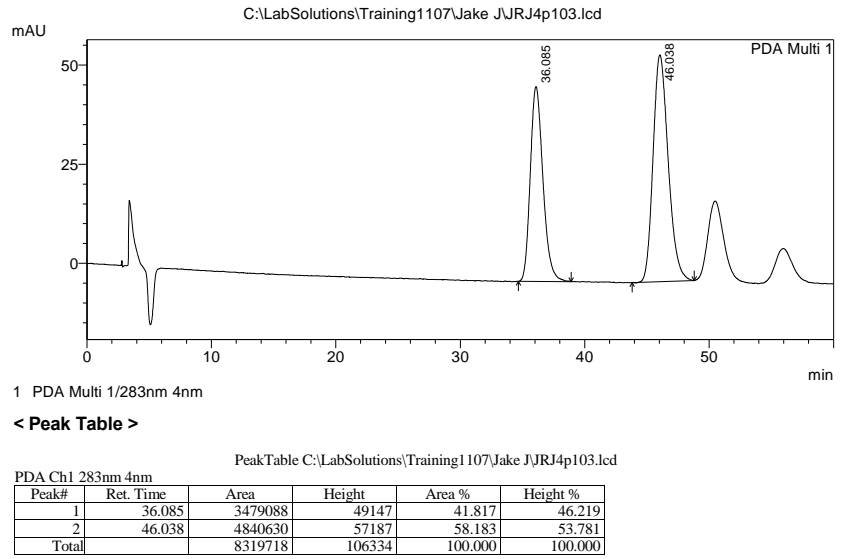


Enantiomerically enriched $(R, R)$ 5e using $R$-TCPTTL:

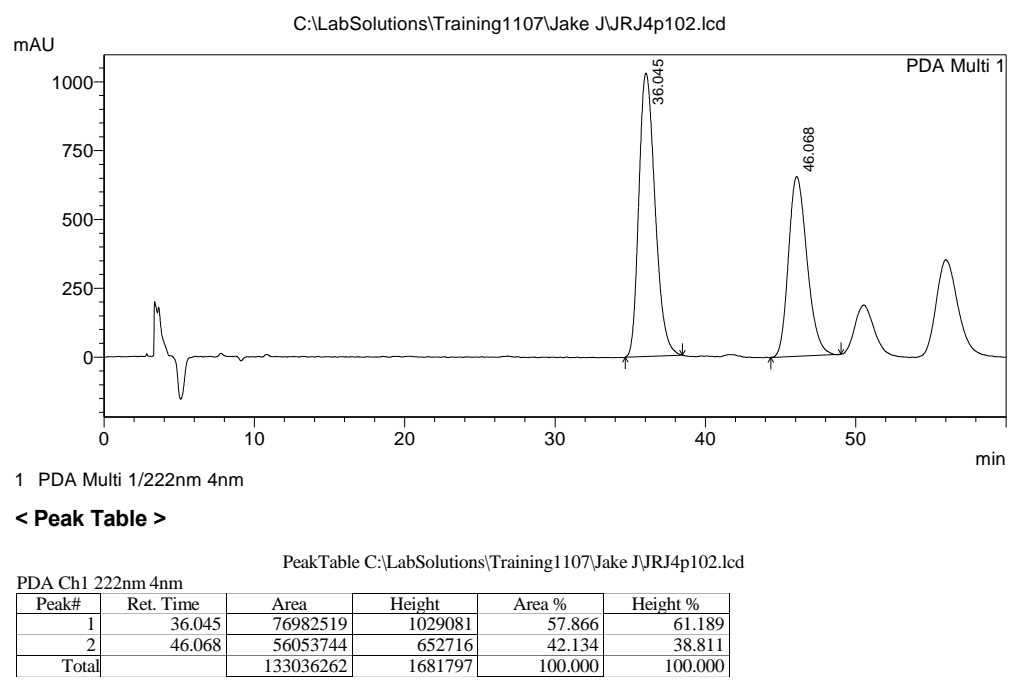




\section{(S)-(4-methoxyphenyl)(methyl)((S)-phenyl(o-tolyl)methyl)silane (5f):}

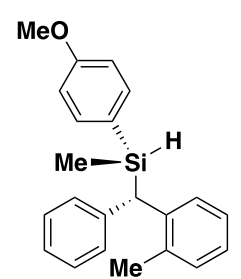

Synthesized using method $F$ with diazo compound $\mathbf{3 a}(0.2 \mathrm{mmol}, 42.0 \mathrm{mg})$ to give a clear oil in $85 \%$ yield $(0.170 \mathrm{mmol}, 56.5 \mathrm{mg}, 94: 6 \mathrm{dr})$. Enantiomeric ratio was determined by HLPC after hydrolysis with a Daicel CHIRALPAK ® AD-H column (1\% IPA/ heptane), $1.0 \mathrm{~mL} / \mathrm{min} . \mathrm{tR}_{\mathrm{R}}(\mathbf{5 f a})=33.3 \mathrm{~min}, \mathrm{tR}_{\mathrm{R}}(\mathbf{5 f b})=37.5 \mathrm{~min}, \mathrm{tR}_{\mathrm{R}}\left(\mathbf{5} \mathbf{f a} \mathbf{a}^{\prime}\right)=47.5 \mathrm{~min}, \mathrm{tR}_{\mathrm{R}}\left(\mathbf{5} \mathbf{f b} \mathbf{b}^{\prime}\right)=56.3$ min, 93.5:6.5 er (Si-OH product, $\mathbf{5 f b}$ ':5fb). Absolute configuration was assigned to be $(S, S)$ based Tamao-Fleming oxidation results and analogy to $\mathbf{1 1 .}$

1H NMR (400 MHz, $\left.\mathrm{CDCl}_{3}\right) \delta 7.39(\mathrm{~d}, J=7.6 \mathrm{~Hz}, 2 \mathrm{H}), 7.04-7.23(\mathrm{~m}, 4 \mathrm{H}), 6.80(\mathrm{~d}, J=7.9 \mathrm{~Hz}, 1 \mathrm{H}), 4.73-4.67(\mathrm{~m}$, $1 \mathrm{H}), 3.93(\mathrm{~d}, J=3.7 \mathrm{~Hz}, 1 \mathrm{H}), 3.79(\mathrm{~s}, 1 \mathrm{H}), 2.22(\mathrm{~s}, 1 \mathrm{H}), 0.31(\mathrm{~d}, J=3.5 \mathrm{~Hz}, 3 \mathrm{H}),{ }_{13} \mathrm{C}$ NMR $\left(151 \mathrm{MHz}, \mathrm{CDCl}_{3}\right) \delta$ 160.9, 142.13, 140.7, 137.0, 136.6, 130.9, 129.7, 128.9, 128.3, 126.0, 125.9, 125.7, 125.2, 113.6, 55.2, 39.0, 20.4, 5.4. $9 \mathrm{Si} \mathrm{NMR}\left(119 \mathrm{MHz}, \mathrm{CDCl}_{3}\right) \delta$-11.4. Did not ionize using ESI, MALDI or APCI.

Racemic Standard for $\mathbf{5 f}$ :

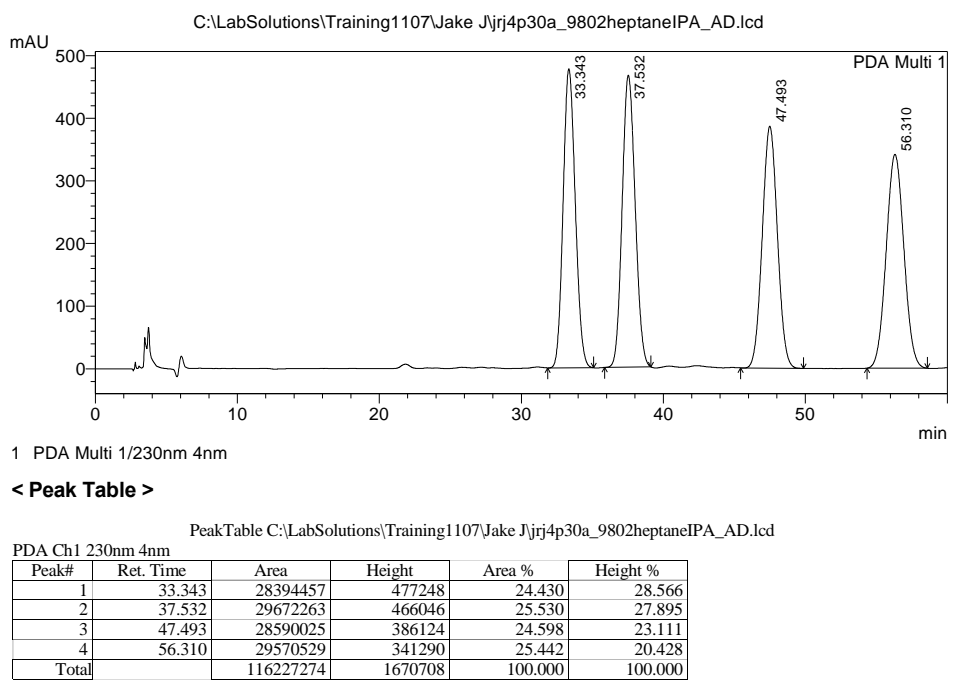

Enantiomerically enriched $(S, S)$ 5f using $S$-TCPTTL:

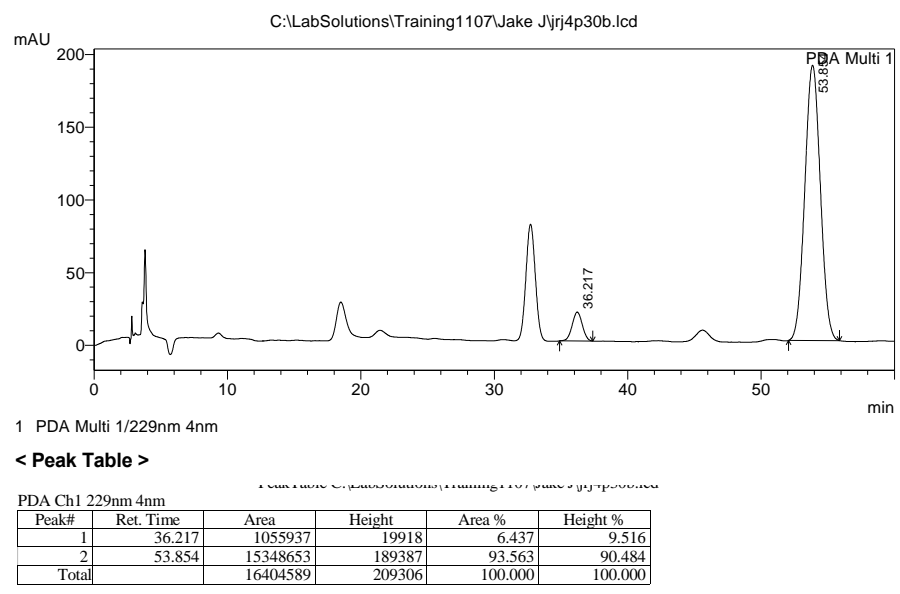


Enantiomerically enriched $(R, R)$ 5f using $R$-TCPTTL:

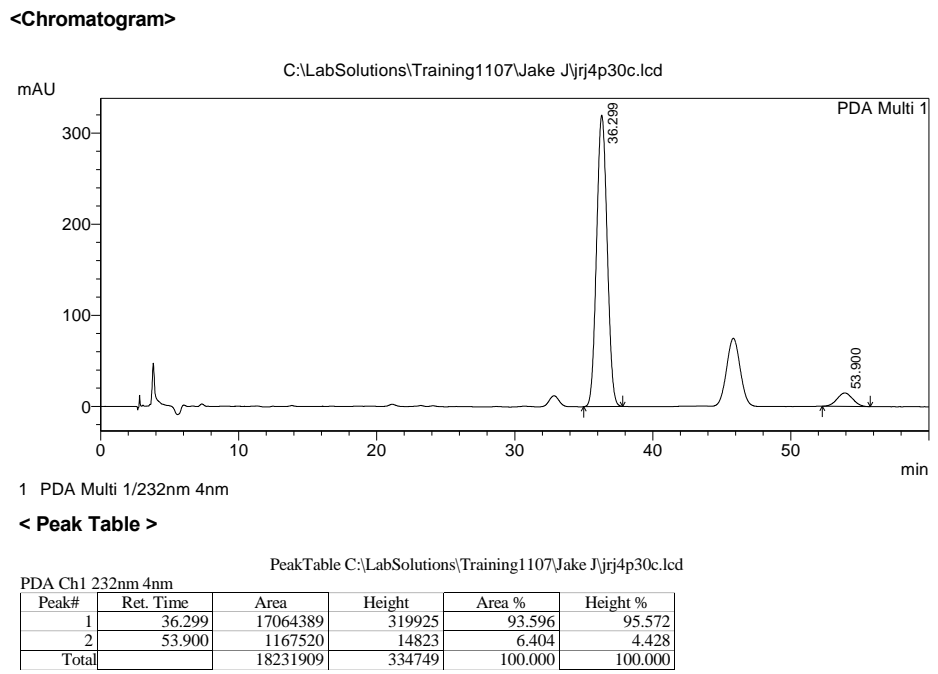




\section{(S)-(4-fluorophenyl)(methyl)((S)-phenyl(o-tolyl)methyl)silane (5g):}

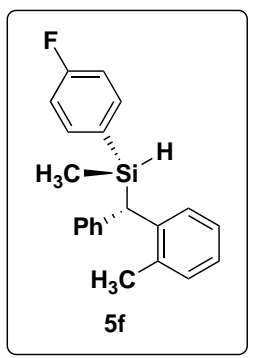

Synthesized using method $\mathrm{F}$ with diazo compound $\mathbf{3 a}(0.2 \mathrm{mmol}, 42.0 \mathrm{mg})$ to give an oil in $90 \%$ yield $(0.180 \mathrm{mmol}, 57.7 \mathrm{mg}, 90: 10 \mathrm{dr})$. Enantiomeric ratio was determined by HLPC after hydrolysis with a Daicel CHIRALPAK ${ }^{\circledR}$ AD-H column (2\% IPA/ hexanes), $1.0 \mathrm{~mL} / \mathrm{min} . \mathrm{tR}_{\mathrm{R}}(\mathbf{5 g a})=36.0 \mathrm{~min}, \mathrm{tR}(\mathbf{5 g b})=46.3 \mathrm{~min}, \mathrm{tR}_{\mathrm{R}}\left(\mathbf{5} \mathbf{g a} \mathbf{9}^{\prime}\right)=50.8 \mathrm{~min}, \mathrm{tR}_{\mathrm{R}}$ $(\mathbf{5 g b} ')=56.5 \mathrm{~min}, 61: 39$ er $(\mathrm{Si}-\mathrm{OH}$ product, $\mathbf{5 g b} \mathbf{5 g b})$ Absolute configuration was assigned to be $(S, S)$ based on analogy to $\mathbf{5 a}$.

1H NMR (600 MHz, CDCl3) $\delta 7.43(\mathrm{dd}, J=7.6,1.4 \mathrm{~Hz}, 1 \mathrm{H}), 7.30-7.23(\mathrm{~m}, 2 \mathrm{H}), 7.23-7.15(\mathrm{~m}, 4 \mathrm{H}), 7.15-7.05$ (m, 4H), $7.01-6.94(\mathrm{~m}, 2 \mathrm{H}), 4.76(\mathrm{p}, J=3.7 \mathrm{~Hz}, 1 \mathrm{H}), 3.96(\mathrm{~d}, J=4.1 \mathrm{~Hz}, 1 \mathrm{H}), 2.24(\mathrm{~s}, 3 \mathrm{H}), 0.37(\mathrm{~d}, J=3.7 \mathrm{~Hz}, 3 \mathrm{H})$. ${ }_{13} \mathrm{C}$ NMR $\left(151 \mathrm{MHz}, \mathrm{CDCl}_{3}\right) \delta 164.1\left(\mathrm{~d}, J_{C F}=248.9 \mathrm{~Hz}\right), 141.8,140.26,137.0\left(\mathrm{~d}, J_{C C C F}=7.5 \mathrm{~Hz}\right), 137.0,130.9$, 129.6, 128.9, 128.5, 128.4, 126.1, 126.0, 125.3, $115.0\left(\mathrm{~d}, J_{C C F}=19.7 \mathrm{~Hz}\right), 38.8,20.3,-5.5 .19 \mathrm{~F} \mathrm{NMR}(376 \mathrm{MHz}$, $\left.\mathrm{CDCl}_{3}\right) \delta$-110.97. $29 \mathrm{Si} \mathrm{NMR}\left(76 \mathrm{MHz}, \mathrm{CDCl}_{3}\right) \delta$-10.9. MALDI $m / z$ calc for $\mathrm{C}_{21} \mathrm{H}_{21} \mathrm{FSi}[\mathrm{M}+\mathrm{Na}]+343.129$. Found 343.134 .

Racemic Standard for $\mathbf{5 g}$ :

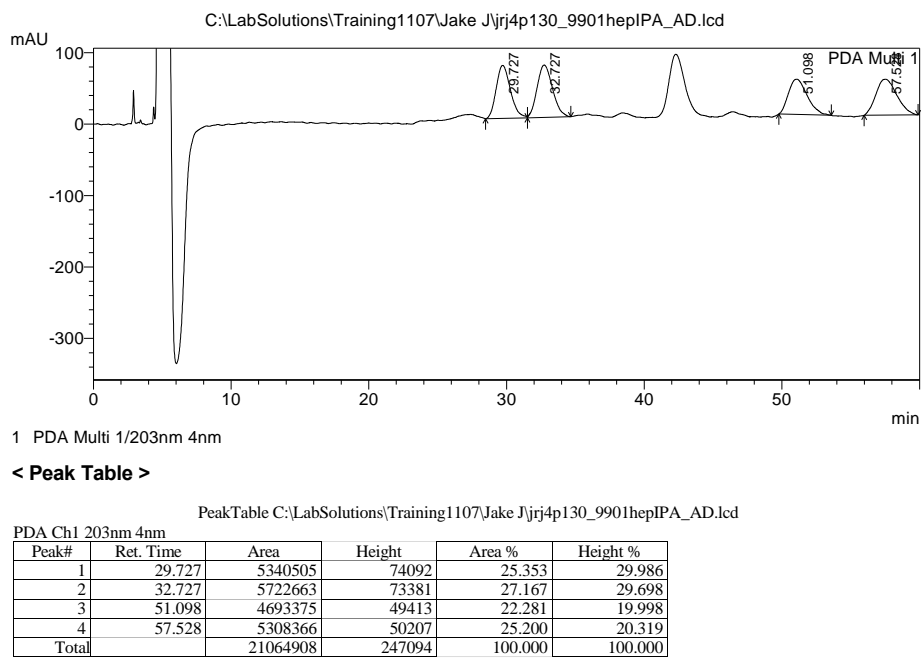

Enantiomerically enriched $(S, S) \mathbf{5 g}$ using $S$-TCPTTL:

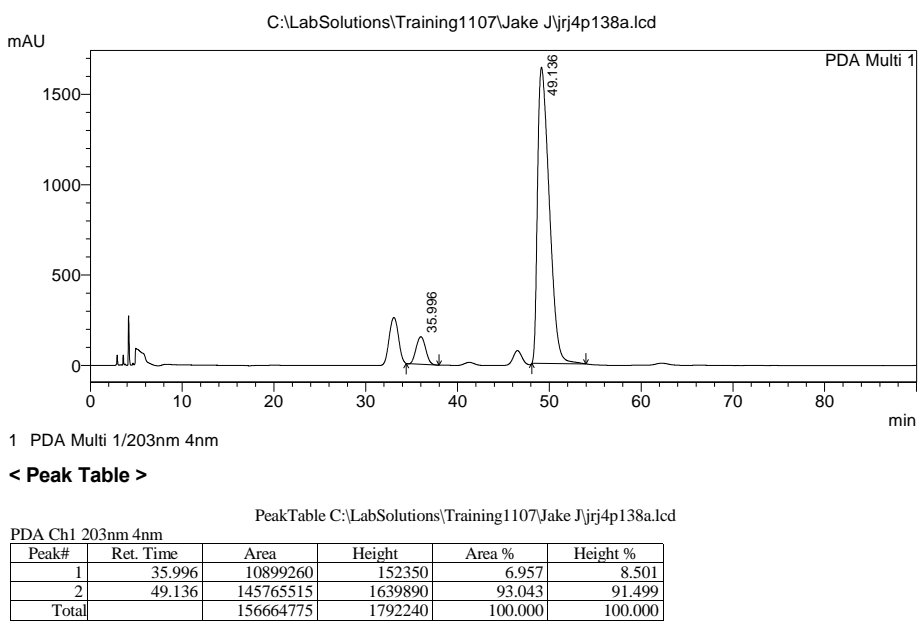


Enantiomerically enriched $(R, R) \mathbf{5 g}$ using $R$-TCPTTL:

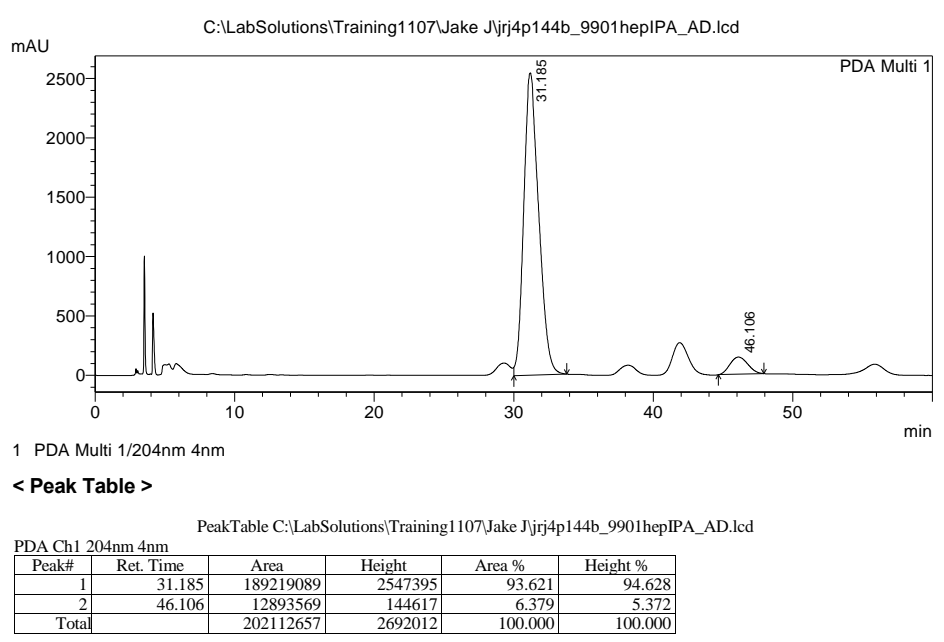




\section{$(S)$-methyl(naphthalen-2-yl)((S)-phenyl(o-tolyl)methyl)silane (5h):}

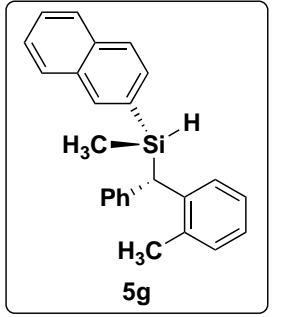

Synthesized using method F with diazo compound 3a $(0.2 \mathrm{mmol}, 42.0 \mathrm{mg})$ to give a white solid in $95 \%$ yield $(0.190 \mathrm{mmol}, 66.9 \mathrm{mg}, 91: 9 \mathrm{dr})$. Enantiomeric ratio was determined by HLPC after hydrolysis with a Daicel CHIRALPAK ® AD-H column $(1 \% \mathrm{IPA} /$ heptane $), 1.0 \mathrm{~mL} / \mathrm{min} . \mathrm{tR}(\mathbf{5 h a})=57.1 \mathrm{~min}, \mathrm{tR}(\mathbf{5 h b})=62.8 \mathrm{~min}, \mathrm{tr}(\mathbf{5 h a})=$ $86.4 \mathrm{~min}, \mathrm{tR}(\mathbf{5 h b})=113.1 \mathrm{~min}, 92: 8$ er $(\mathrm{Si}-\mathrm{OH}$ product, 5hb':5hb) Absolute configuration was assigned to be $(S, S)$ based on analogy to $\mathbf{5 a}$.

1H NMR (600 MHz, CDCl $) \delta 7.85(\mathrm{~m}, 2 \mathrm{H}), 7.77(\mathrm{dd}, J=12.3,8.0 \mathrm{~Hz}, 2 \mathrm{H}), 7.55-7.48(\mathrm{~m}, 3 \mathrm{H}), 7.35(\mathrm{~d}, J=8.1 \mathrm{~Hz}$, 1H), $7.21(\mathrm{td}, J=15.6,14.8,7.9 \mathrm{~Hz}, 4 \mathrm{H}), 7.17-7.09(\mathrm{~m}, 4 \mathrm{H}), 4.93(\mathrm{p}, J=3.8 \mathrm{~Hz}, 1 \mathrm{H}), 4.11(\mathrm{~d}, J=3.7 \mathrm{~Hz}, 1 \mathrm{H}), 2.29$ (s, 3H), $0.48(\mathrm{~d}, J=3.5 \mathrm{~Hz}, 3 \mathrm{H}) .{ }_{13} \mathrm{C}$ NMR $\left(151 \mathrm{MHz}, \mathrm{CDCl}_{3}\right) \delta 141.9,140.5,137.0,136.2,134.0,132.9,132.5$, 131.0, 130.9, 129.7, 128.9, 128.4, 128.2, 127.8, 126.9, 126.7, 126.1, 126.0, 126.0, 125.3, 38.8, 20.4, -5.4. 29Si NMR $\left(119 \mathrm{MHz}, \mathrm{CDCl}_{3}\right) \delta$-10.1. MALDI $m / z$ calc for $\mathrm{C}_{25} \mathrm{H}_{24} \mathrm{Si}[\mathrm{M}+\mathrm{Na}]+375.154$. Found 375.166.

Racemic Standard for $\mathbf{5 h}$ :

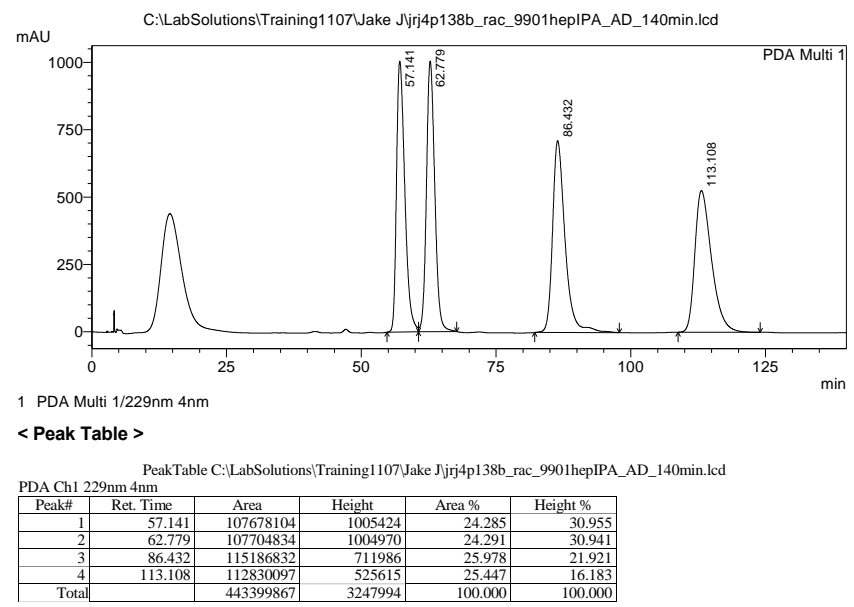

Enantiomerically enriched $(S, S)$ 5h using $S$-TCPTTL:

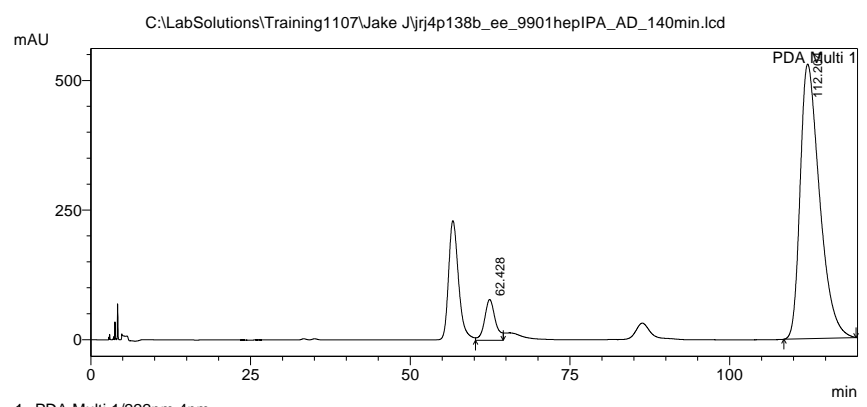

1 PDA Multi $1 / 222 n m 4 n m$

<Peak Table >

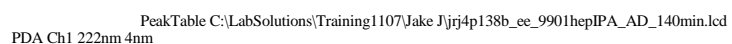

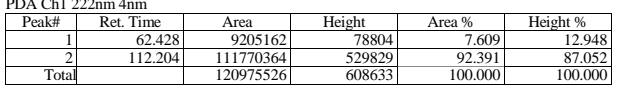


Enantiomerically enriched $(R, R) \mathbf{5 h}$ using $R$-TCPTTL:

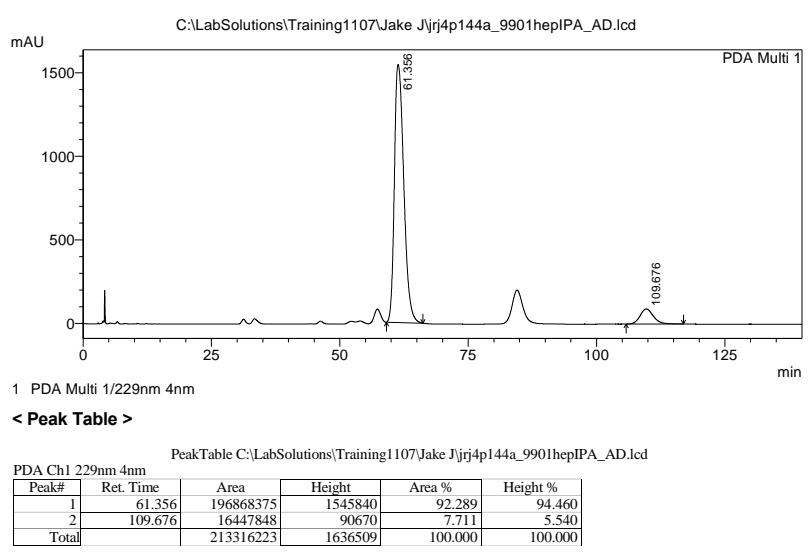




\section{$(S)$-methyl(naphthalen-1-yl)((S)-phenyl(o-tolyl)methyl)silane (5i):}

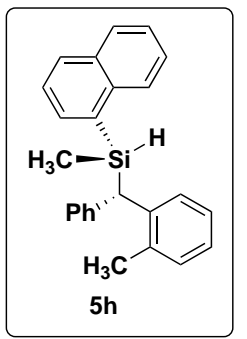

Synthesized using method $\mathrm{F}$ with diazo compound 3a $(0.2 \mathrm{mmol}, 42.0 \mathrm{mg})$ to give a white solid in $55 \%$ yield $(0.11 \mathrm{mmol}, 38.8 \mathrm{mg}, 82: 18 \mathrm{dr}$. Enantiomeric ratio was determined by HLPC after hydrolysis with a Daicel CHIRALPAK ® AD-H column ( $2 \%$ IPA/ heptane $), 1.0 \mathrm{~mL} / \mathrm{min}$. $\mathrm{tR}(\mathbf{5 i a})=23.9 \mathrm{~min}, \mathrm{tR}(\mathbf{5 i b})=25.5 \mathrm{~min}, \mathrm{tR}(\mathbf{5 i a})=41.6 \mathrm{~min}$, tR $(\mathbf{5 i b}$ ') $=50.4 \mathrm{~min}, 92: 8$ er $(\mathrm{Si}-\mathrm{OH}$ product, 5ia':5ia) Absolute configuration was assigned to be $(S, S)$ based on analogy to $\mathbf{5 a}$.

1H NMR (400 MHz, CDCl3) $\delta 7.90-7.75(\mathrm{~m}, 4 \mathrm{H}), 7.57(\mathrm{~d}, J=6.7 \mathrm{~Hz}, 1 \mathrm{H}), 7.53-7.38(\mathrm{~m}, 3 \mathrm{H}), 7.34(\mathrm{dt}, J=8.5$, $4.5 \mathrm{~Hz}, 2 \mathrm{H}), 7.15(\mathrm{t}, J=7.2 \mathrm{~Hz}, 2 \mathrm{H}), 7.08(\mathrm{~m}, 2 \mathrm{H}), 7.02(\mathrm{~m}, 1 \mathrm{H}), 6.95(\mathrm{~m}, 1 \mathrm{H}), 5.16(\mathrm{p}, J=4.1 \mathrm{~Hz}, 1 \mathrm{H}), 4.23(\mathrm{~d}, J=$ $4.5 \mathrm{~Hz}, 1 \mathrm{H}), 2.17(\mathrm{~s}, 3 \mathrm{H}), 0.45(\mathrm{~d}, J=3.8 \mathrm{~Hz}, 3 \mathrm{H}) . \mathrm{NMR}\left(101 \mathrm{MHz}, \mathrm{CDCl}_{3}\right) \delta 142.3,14.0,137.2,136.9,135.2,133.5$, 133.3, 130.9, 130.4, 129.8, 127.0, 128.7, 128.3, 127.7, 126.1, 126.0, 125.9, 125.6, 125.2, 125.1, 38.5, 20.3, -4.64.29 Si NMR $\left(119 \mathrm{MHz}, \mathrm{CDCl}_{3}\right) \delta$-10.6. MALDI $m / z$ calc for $\mathrm{C}_{25} \mathrm{H}_{24} \mathrm{Si}[\mathrm{M}+\mathrm{Na}]+375.154$. Found 375.163.

Racemic Standard for 5i:

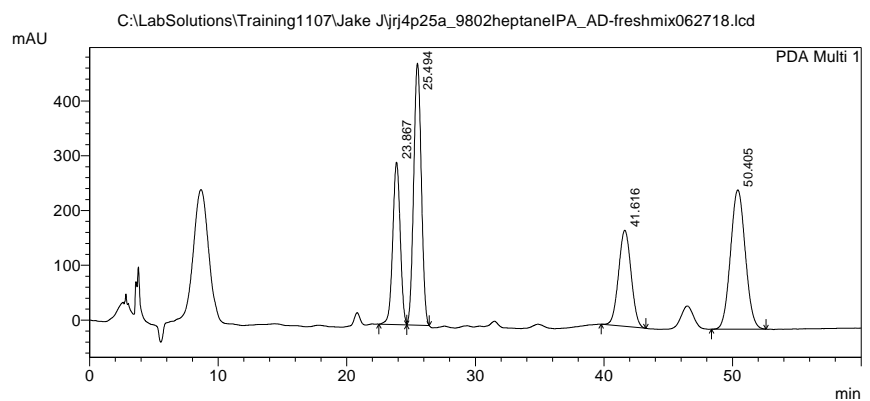

1 PDA Multi $1 / 225 \mathrm{~nm} 4 \mathrm{~nm}$

< Peak Table >

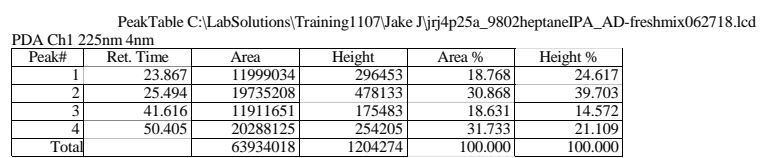

Enantiomerically enriched $(S, S)$ 5i using $S$-TCPTTL:

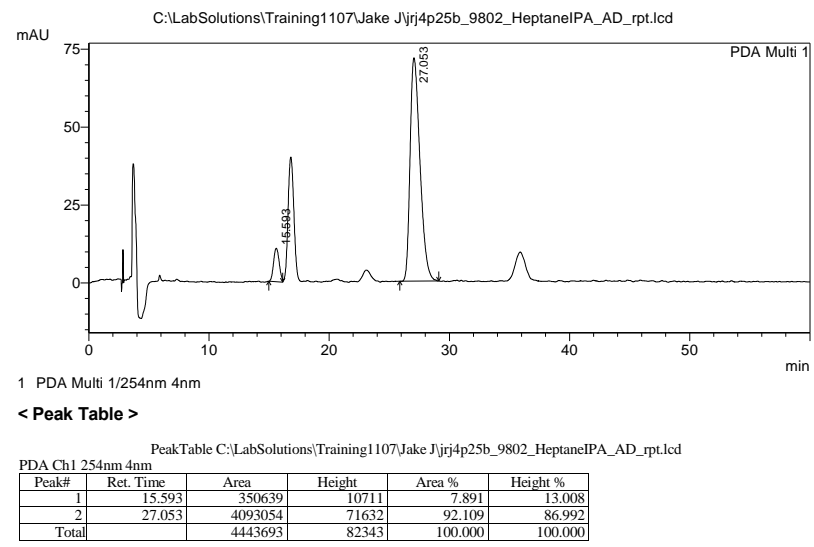




\section{(S)-(4-fluoronaphthalen-1-yl)(methyl)((S)-phenyl(o-tolyl)methyl)silane (5j):}

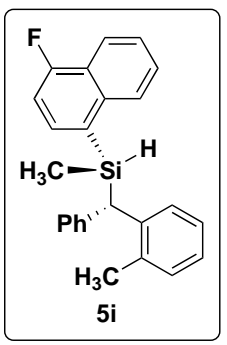

Synthesized using method $\mathrm{F}$ with diazo compound $\mathbf{3 a}(0.2 \mathrm{mmol}, 42.0 \mathrm{mg})$ to give a white solid in $66 \%$ yield $(0.132 \mathrm{mmol}, 48.9 \mathrm{mg}, 84: 16 \mathrm{dr}$. Enantiomeric ratio was determined by HLPC after hydrolysis with a Daicel CHIRALPAK ® AD-H column (2\% IPA/ heptane $), 1.0 \mathrm{~mL} / \mathrm{min}$. tR $(\mathbf{5 j a})=35.0 \mathrm{~min}, \mathrm{tR}_{\mathrm{a}}(\mathbf{5 j b})=37.9 \mathrm{~min}, \mathrm{tR}_{\mathrm{R}}\left(\mathbf{5} \mathbf{j} \mathbf{j} \mathbf{\prime}^{\prime}\right)=59.8 \mathrm{~min}, \mathrm{t}_{\mathrm{R}}$ $(\mathbf{5 j b})=65.6 \mathrm{~min}, 92: 8 \mathrm{er}(\mathrm{Si}-\mathrm{OH}$ product, 5ja':5ja) Absolute configuration was assigned to be $(S, S)$ based on analogy to $\mathbf{5 a}$.

${ }_{1} \mathrm{H}$ NMR $\left(400 \mathrm{MHz}, \mathrm{CDCl}_{3}\right) \delta 8.18(\mathrm{~d}, J=8.3 \mathrm{~Hz}, 1 \mathrm{H}), 7.94(\mathrm{~d}, J=8.5 \mathrm{~Hz}, 1 \mathrm{H}), 7.59-7.42(\mathrm{~m}, 4 \mathrm{H}), 7.25-7.00(\mathrm{~m}$, $9 \mathrm{H}), 5.20(\mathrm{q}, J=5.9,4.0 \mathrm{~Hz}, 1 \mathrm{H}), 4.24(\mathrm{~d}, J=4.4 \mathrm{~Hz}, 1 \mathrm{H}), 2.23(\mathrm{~s}, 3 \mathrm{H}), 0.52(\mathrm{~d}, J=3.5 \mathrm{~Hz}, 3 \mathrm{H}) .13 \mathrm{C}$ NMR $(101$ $\left.\mathrm{MHz}, \mathrm{CDCl}_{3}\right) \delta 160.5\left(\mathrm{~d}, J_{C F}=255.0 \mathrm{~Hz}\right), 140.2,136.8,135.2\left(\mathrm{~d}, J_{C C C F}=8.3 \mathrm{~Hz}\right), 130.9,129.66,129.1\left(\mathrm{~d}, J_{C C C F}=\right.$ $4.9 \mathrm{~Hz}), 128.7,128.6,128.4,128.2,127.4\left(\mathrm{~d}, J_{C C C} F=3.2 \mathrm{~Hz}\right), 126.8,126.0,125.9,125.8\left(\mathrm{~d}, J_{C C C C F}=1.6 \mathrm{~Hz}\right), 125.1$, $123.7\left(\mathrm{~d}, J_{C C F}=15.0 \mathrm{~Hz}\right), 121.1\left(\mathrm{~d}, J_{C C C F}=6.3 \mathrm{~Hz}\right), 108.9\left(\mathrm{~d}, J_{\mathrm{CCF}}=18.5 \mathrm{~Hz}\right), 38.5,20.2,-4.8 .19 \mathrm{~F} \mathrm{NMR}(376 \mathrm{MHz}$, $\left.\mathrm{CDCl}_{3}\right) \delta$-120.35. ${ }_{29} \mathrm{Si} \mathrm{NMR}\left(76 \mathrm{MHz}, \mathrm{CDCl}_{3}\right) \delta-12.7 \mathrm{MALDI} \mathrm{m} / z$ calc for $\mathrm{C}_{25} \mathrm{H}_{23} \mathrm{FSi}[\mathrm{M}+\mathrm{H}]+371.163$. Found 371.151 .

Racemic Standard for $\mathbf{5 j}$ :

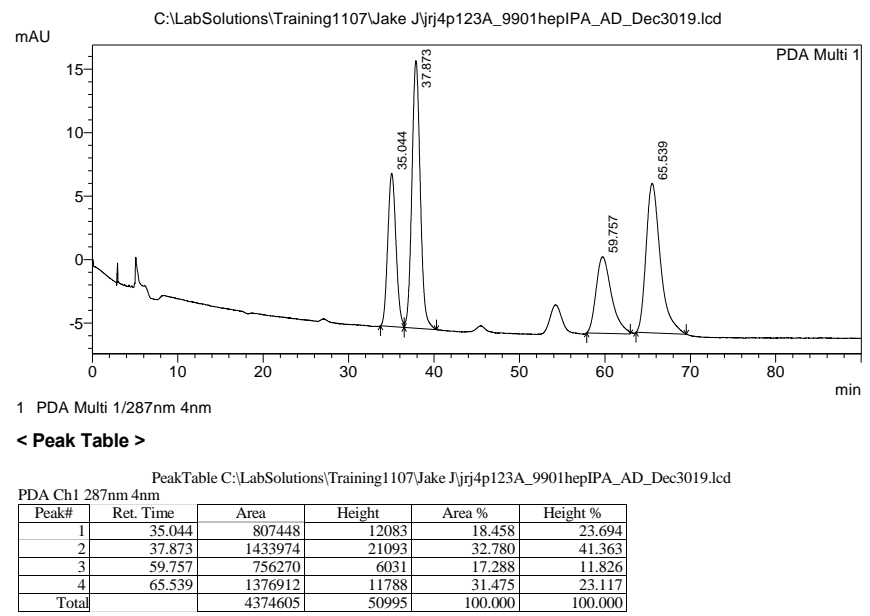

Enantiomerically enriched $(S, S) \mathbf{5 j}$ using $S$-TCPTTL:

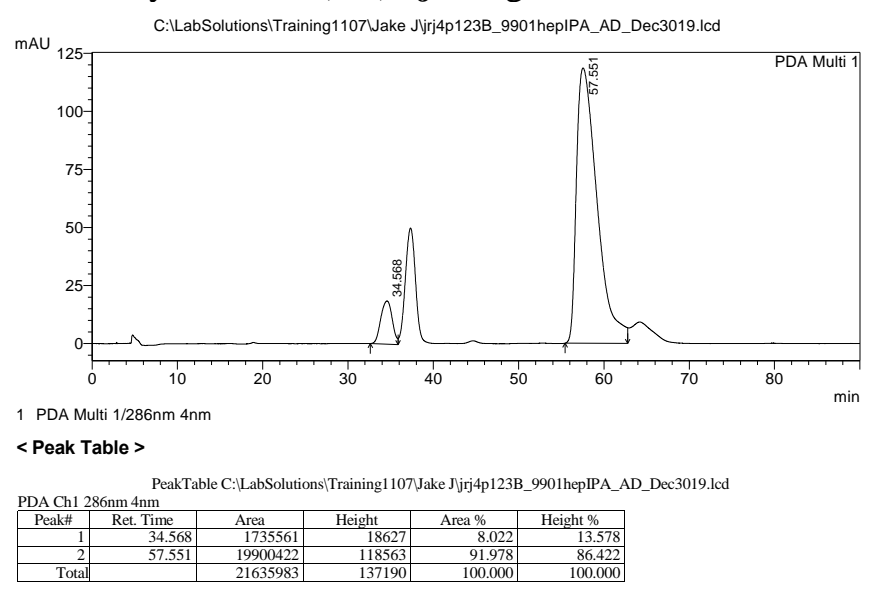




\section{Functionalization Products and Miscellaneous:}

\section{1,2-bis(diphenylmethylene)hydrazine (6):}

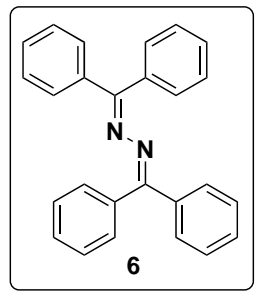

Spectrum matches previous report.17

${ }_{1} \mathrm{H}$ NMR $\left(600 \mathrm{MHz}, \mathrm{CDCl}_{3}\right) \delta 7.47(\mathrm{~d}, J=7.7 \mathrm{~Hz}, 4 \mathrm{H}), 7.39(\mathrm{dt}, J=11.4,6.5 \mathrm{~Hz}, 6 \mathrm{H}), 7.36-7.31(\mathrm{~m}, 6 \mathrm{H}), 7.27(\mathrm{t}$, $J=7.5 \mathrm{~Hz}, 4 \mathrm{H})$.

(1E,2E)-1,2-bis(phenyl(o-tolyl)methylene)hydrazine (7):

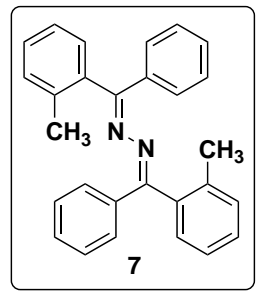

Observed and isolated during kinetics experiments as a yellow solid.

1H NMR (600 MHz, CDCl 3 ) $\delta 7.37(\mathrm{~m}, 4 \mathrm{H}), 7.29(\mathrm{~m}, 6 \mathrm{H}), 7.22(\mathrm{~m}, 6 \mathrm{H}), 2.23(\mathrm{~s}, 6 \mathrm{H}),{ }_{13} \mathrm{C}$ NMR $\left(151 \mathrm{MHz}, \mathrm{CDCl}_{3}\right)$ $\delta 137.8,136.6,130.3,123.0,129.9,128.3,128.3,128.2,128.0,125.4,20.2$ 


\section{$(R)$-methyl(phenyl)((S)-phenyl(o-tolyl)methyl)silanol (9):}

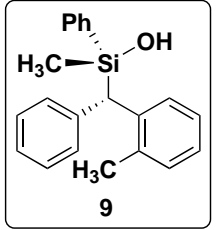

Synthesized using method K $\mathbf{5 a}(0.33 \mathrm{mmol}, 100.0 \mathrm{mg})$ to give a clear oil in $90 \%$ yield (0.3 mmol, $94.5 \mathrm{mg}, 93: 7 \mathrm{dr}$ ). Enantiomeric ratio was determined by HLPC with a Daicel CHIRALPAK $®$ AD-H column (1\% IPA/ heptane), $1.0 \mathrm{~mL} / \mathrm{min} . \mathrm{t}_{\mathrm{R}}(\mathbf{9 a})=29.9 \mathrm{~min}, \mathrm{t}_{\mathrm{R}}$ $(\mathbf{9 b})=32.1 \mathrm{~min}, \mathrm{tR}\left(\mathbf{9} \mathbf{a}^{\prime}\right)=45.3 \mathrm{~min}, \mathrm{tR}\left(\mathbf{9} \mathbf{b}^{\prime}\right)=53.3 \mathrm{~min}, 93: 7 \mathrm{er}\left(\mathbf{9 b}: 9 \mathbf{b}^{\prime}\right)$. Absolute configuration was assigned to be $(R, S)$ based on analogy to $\mathbf{5 a}$, and formed through a retentive process.11

${ }_{1} \mathrm{H}$ NMR (400 MHz, C6D6) $\delta 7.70(\mathrm{~d}, J=7.6 \mathrm{~Hz}, 1 \mathrm{H}), 7.36(\mathrm{~d}, J=7.9 \mathrm{~Hz}, 2 \mathrm{H}), 7.26(\mathrm{~d}, J=7.6 \mathrm{~Hz}, 2 \mathrm{H}), 7.12-7.06$ $(\mathrm{m}, 5 \mathrm{H}), 7.05-6.88(\mathrm{~m}, 4 \mathrm{H}), 3.96(\mathrm{~s}, 1 \mathrm{H}), 2.03(\mathrm{~s}, 3 \mathrm{H}), 1.57(\mathrm{~s}, 1 \mathrm{H}), 0.29(\mathrm{~s}, 1 \mathrm{H}) .{ }_{13 \mathrm{C} \mathrm{NMR}}(151 \mathrm{MHz}, \mathrm{CDCl} 3) \delta$ 141.3, 139.7, 137.1, 136.9, 134.0, 130.9, 130.0, 129.9, 129.0, 128.4, 127.8, 126.0, 125.9, 125.4, 41.6, 20.4, -1.6. 29Si NMR $\left(79 \mathrm{MHz}, \mathrm{CDCl}_{3}\right) \delta$ 1.66. MALDI $\mathrm{m} / z$ calc for $\mathrm{C}_{22} \mathrm{H}_{22} \mathrm{Si}[\mathrm{M}+\mathrm{Na}]+341.1332$. Found 341.212.

Racemic Standard for 9:

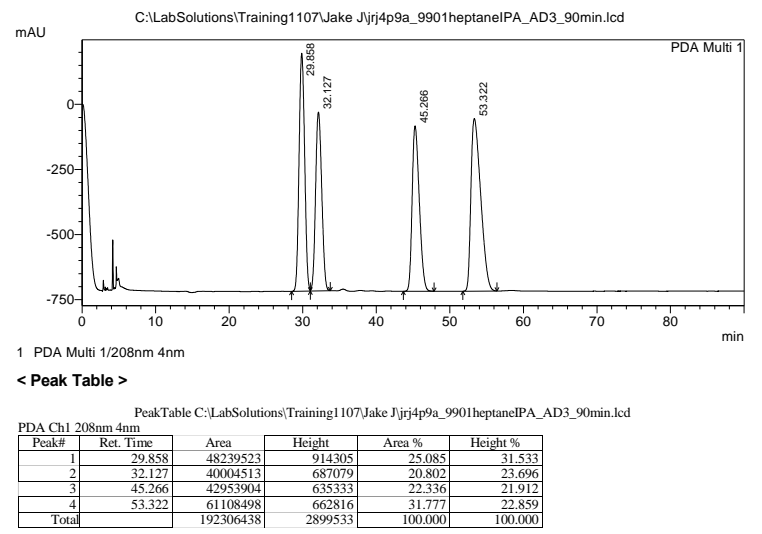

Enantiomerically enriched $(S, S) 9$ using $S$-TCPTTL:

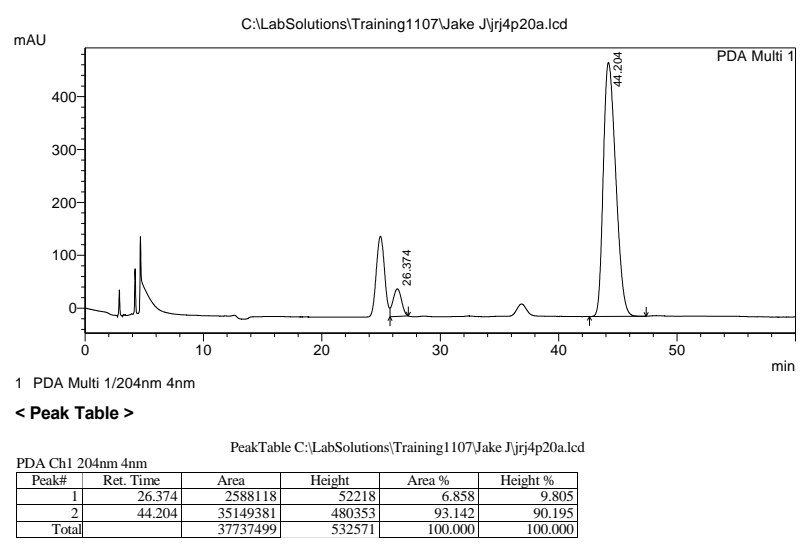




\section{$(R)-((E)-4-f l u o r o s t y r y l)(m e t h y l)($ phenyl $)((S)$-phenyl(o-tolyl)methyl)silane (10):}

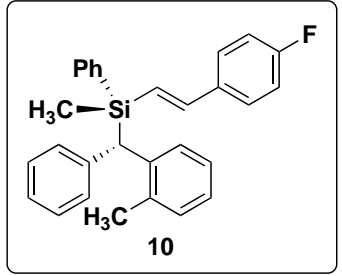

Synthesized using method L with $\mathbf{5 a}(0.33 \mathrm{mmol}, 100.0 \mathrm{mg})$ to give a clear oil in $62 \%$ yield $(104.1 \mathrm{mg}, 85: 15$ 10:10', $93: 7 \mathrm{dr})$. Absolute configuration was assigned to be $(R, S)$ based on analogy to $\mathbf{5 a}$ and assuming retention of configuration.11 The $E$ aisomer was assigned based off $J$ values of the alkene protons. Could not separate using CSP-HPLC.

${ }_{1} \mathrm{H}$ NMR $\left(600 \mathrm{MHz}_{\mathrm{CDCl}}\right) \delta 7.41-7.32(\mathrm{~m}, 6 \mathrm{H}), 7.29(\mathrm{~m} 2 \mathrm{H}), 7.20-7.13(\mathrm{~m}, 3 \mathrm{H}), 7.13-7.06(\mathrm{~m}, 4 \mathrm{H}), 7.02(\mathrm{~m}$, $3 \mathrm{H}), 6.81(\mathrm{~d}, J=19.2 \mathrm{~Hz}, 1 \mathrm{H}), 6.50(\mathrm{~d}, J=19.2 \mathrm{~Hz}, 1 \mathrm{H}), 4.11(\mathrm{~s}, 1 \mathrm{H}), 2.22(\mathrm{~s}, 3 \mathrm{H}), 0.46(\mathrm{~s}, 3 \mathrm{H}) .13 \mathrm{C} \mathrm{NMR}(151 \mathrm{MHz}$, $\left.\mathrm{CDCl}_{3}\right) \delta 162.9\left(\mathrm{~d}, J_{C F}=250.4 \mathrm{~Hz}\right), 145.8,141.7,140.5,137.0,136.8,135.0,130.9,130.3,129.4,129.1,128.9,128.4$, $128.3,128.2,127.8,125.8\left(\mathrm{~d}, J_{C C C F}=6.3 \mathrm{~Hz}\right), 125.1,124.1\left(\mathrm{~d}, J_{C C C C F}=2.3 \mathrm{~Hz}\right), 115.6\left(\mathrm{~d}, J_{\mathrm{CCF}}=21.6 \mathrm{~Hz}\right), 40.1,20.4$, -3.9.MALDI $m / z$ calc for $\mathrm{C}_{29} \mathrm{H}_{27} \mathrm{FSi}\left[\mathrm{M}+\mathrm{H}_{3} \mathrm{O}\right]+$. 441.204. Found 441.158. ${ }_{19} \mathrm{~F}$ NMR $\left(376 \mathrm{MHz} \mathrm{CDCl}_{3}\right) \delta-113.3$. 29: $\mathrm{Si}$ NMR (79 $\left.\mathrm{MHz}, \mathrm{CDCl}_{3}\right) \delta-12.0$.

\section{(1S,2R)-2-methyl-1,2-diphenyl-2,3-dihydro-1H-benzo[c]silole (11):}

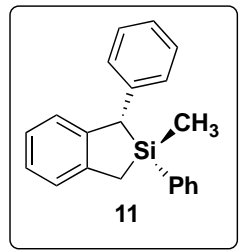

Synthesized using method $\mathbf{J}$ with $\mathbf{5 a}(0.33 \mathrm{mmol}, 100.0 \mathrm{mg})$ to give a clear oil in $90 \%$ yield $(0.3 \mathrm{mmol}, 89.2 \mathrm{mg}, 90: 10 \mathrm{dr})$. Absolute configuration was assigned to be $(S, S)$ based on analogy to $\mathbf{5 f}$, and ${ }_{1} \mathrm{H}$ NOESY experiments. Could not separate using CSP-HPLC.

${ }_{1} \mathrm{H}$ NMR $\left(600 \mathrm{MHz}_{\mathrm{CDCl}}\right) \delta 7.46(\mathrm{~d}, J=7.6 \mathrm{~Hz}, 1 \mathrm{H}), 7.32-7.20(\mathrm{~m}, 1 \mathrm{H}), 7.19-7.12(\mathrm{~m}, 4 \mathrm{H}), 7.08(\mathrm{~d}, J=7.6 \mathrm{~Hz}$, 2H), $7.01(\mathrm{~d}, J=7.2 \mathrm{~Hz}, 1 \mathrm{H}), 6.75(\mathrm{~d}, J=8.4 \mathrm{~Hz}, 2 \mathrm{H}), 3.91(\mathrm{~s}, 1 \mathrm{H}), 2.66(\mathrm{~d}, J=17.3 \mathrm{~Hz}, 1 \mathrm{H}), 2.34(\mathrm{~d}, J=17.3 \mathrm{~Hz}$, 1H), 0.63 (s, 3H).13C NMR (151 MHz, $\left.\mathrm{CDCl}_{3}\right) \delta 144.8,142.9,142.1,134.5,129.6,129.0,128.9,128.1,127.9,127.6$, 126.6, 124.3, 43.4, 18.9, -4.6. $29 \mathrm{Si} \mathrm{NMR}\left(79 \mathrm{MHz}_{\mathrm{CDCl}}\right) \delta$ 15.7. MALDI $m / z$ calc for $\mathrm{C}_{21} \mathrm{H}_{20} \mathrm{Si}[\mathrm{M}+\mathrm{Na}]+323.123$. Found 323.166.

\section{(S)-phenyl(o-tolyl)methanol (S1)}

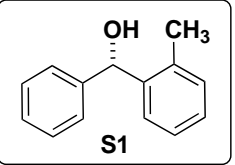

Synthesized using procedure for Tamao-Fleming oxidation.HPLC retention times match previously reported syntheses. Enantiomeric ratio was determined by HLPC with a Daicel CHIRALPAK $®$ OD-H column $(2 \%$ IPA/ hexanes $), 0.5 \mathrm{~mL} / \mathrm{min} . \mathrm{tR}($ S1a $)=20.4 \mathrm{~min}, \mathrm{tR}$ $(\mathbf{S 1 b})=21.6 \mathrm{~min}, 91.5: 8.5$ er. Absolute configuration was assigned to be $(S)$ based on previous reports. 10

${ }_{1} \mathrm{H}$ NMR $\left(600 \mathrm{MHz}, \mathrm{CDCl}_{3}\right) \delta 7.50(\mathrm{~d}, J=7.6 \mathrm{~Hz}, 1 \mathrm{H}), 7.31(\mathrm{~m}, 4 \mathrm{H}), 7.27-7.21(\mathrm{~m}, 2 \mathrm{H}), 7.19(\mathrm{td}, J=7.4,1.6 \mathrm{~Hz}$, $1 \mathrm{H}), 7.13(\mathrm{~d}, J=7.3 \mathrm{~Hz}, 1 \mathrm{H}), 5.97(\mathrm{~s}, 1 \mathrm{H}), 2.23(\mathrm{~s}, 3 \mathrm{H})$. 
Racemic Standard:

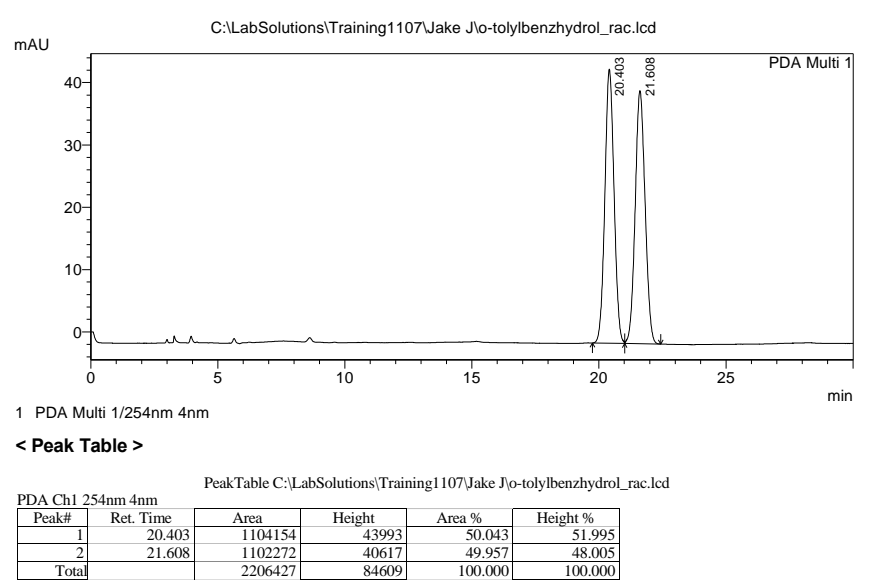

HPLC trace after Tamao-Fleming Oxidation of $\mathbf{5 f}$ :

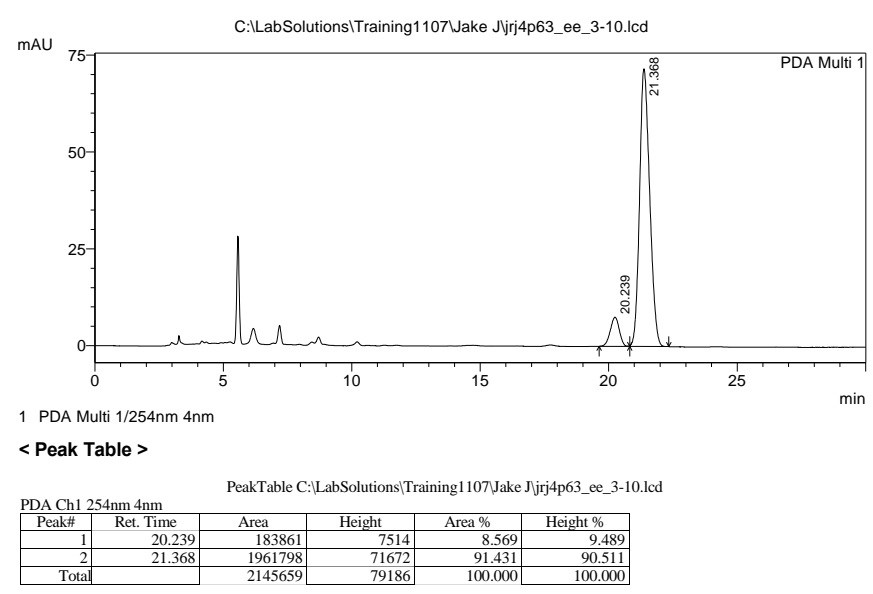




\section{X-ray Crystallography Data}

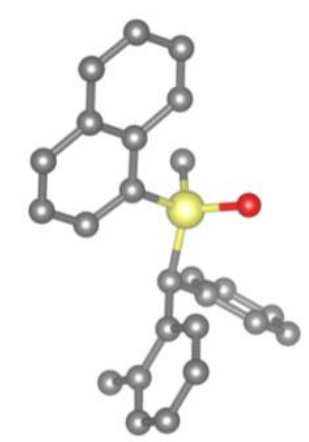

Figure S15. X-ray18 of (+/-) 5i hydrolysis product; H's are removed for clarity as well as the distorted enantiomer.

Crystals were grown from slow diffusion in DCM/Benzene. A colorless plate with approximate orthogonal dimensions $0.282 \times 0.425 \times 0.755 \mathrm{~mm} 3$ was placed and optically centered on the Bruker Duo 19 APEXII CCD system at $-183^{\circ} \mathrm{C}(90 \mathrm{~K})$. Indexing of the unit cell used a random set of reflections collected from three series of $0.5^{\circ}$ wide $\omega$-scans, 10 seconds per frame, and 30 frames per series that were well distributed in reciprocal space. Data were collected $[\mathrm{MoK} \alpha]$ with $0.3^{\circ}$ wide scans, variable time per frame dependent upon detector $2 \theta$ angle and varying $\varphi$ and omega angles such that nearly all unique reflections were collected at least once. The crystal to detector distance was $5.00 \mathrm{~cm}$, thus providing a complete sphere of data to $2 \theta_{\max }=54.97^{\circ}$.

\section{Structural determination and Refinement:}

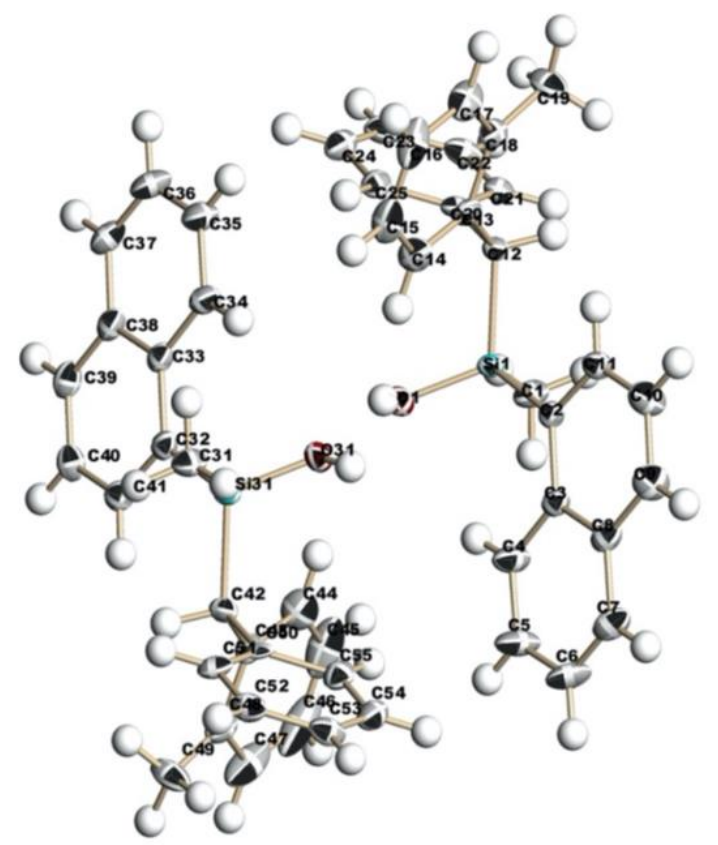

Figure S16. ORTEP of (+/-) 5i hydrolysis product. Thermal Ellipsoids are at 50\%.

All crystallographic calculations were performed on an Intel Xeon E5-1620v2 at 3.70GHz with eight core processor and 16GB of extended memory. Determination of the unit cell required Cellnow 20 and the reciprocal lattice viewing 
(Rlatt) capabilities of the APEX2 program to determine the true lattice. Cellnow20 determined the twin relationship between the two components, a rotation of $179.8^{\circ}$ about the real axis $10-1$ produced the transformation matrix -0.344 $0-0.6500-10-1.35600 .344$, generated the orientation matrices for the components and output a useable multiple matrices input file for the integration program SAINT 19. Saint was run six times using the output optimized merged matrix file from the previous run. Data collected were now corrected for absorption using TWINABS 21,22 and Blessing's method and merged generating both single component HKLF4 and multi-component HKLF5 files. The SHELXTL23 program package was now implemented to determine, based upon systematic absences and intensity statistics, the non-centrosymmetric monoclinic space group P2 ${ }_{1}$ (no. 4). The structure was determined by direct methods with a majority of the non-hydrogen atoms from the two nearly identical molecules being located directly using the program XT.24 Refinement of the structure was achieved using the program XL.25 One of the ortho-methylbenzyl groups was found to be disordered over two positions in one of the molecules, while the other molecule was fully ordered. The $\mathrm{OH}$ groups were also found to be 50\% disordered and optimized with one hydrogen directed towards the other molecules hydroxyl oxygen and the other away for each molecule. The data collected were merged for least squares refinement to 8983 unique data. All non-hydrogen atoms were refined anisotropically. Hydrogen atoms were idealized but their thermal parameters were allowed to refine freely but for those attached to the oxygen atoms; those were fixed to be equal to each other. The final structure was refined to convergence with $\mathrm{R}(\mathrm{F})=3.49 \%$, $\mathrm{wR}\left(\mathrm{F}^{2}\right)=9.64 \%, \mathrm{GOF}=1.043$ for all 8893 unique reflections $\left[\mathrm{R}(\mathrm{F})=3.33, \mathrm{wR}\left(\mathrm{F}^{2}\right)=9.49 \%\right.$ for those 8671 data with Fo $>4 \sigma(\mathrm{Fo})]$. The final difference-Fourier map was featureless indicating that the structure is both correct and complete. The absolute configuration structure parameter, Flack(x),26 was determined to be $0.06(7)$ indicating that the structure is correct. 
Table S4. Crystal data and structure refinement for [ $\left.\mathrm{C}_{25} \mathrm{H}_{24} \mathrm{OSi}\right]$.

Identification code

Empirical formula

Formula weight

Temperature

Wavelength

Crystal system

Space group

Unit cell dimensions

Volume

Z, Z'

Density (calculated)

Absorption coefficient

$\mathrm{F}(000)$

Crystal size

Crystal color and habit

Diffractometer

Theta range for data collection

Index ranges

Reflections collected

Independent reflections

Observed reflections (I $>2$ sigma(I))

Completeness to theta $=25.242^{\circ}$

Absorption correction

Max. and min. transmission

Solution method

Refinement method

Data / restraints / parameters

Goodness-of-fit on $\mathrm{F}^{2}$

Final $\mathrm{R}$ indices [I $>2 \operatorname{sigma}(\mathrm{I})]$

$\mathrm{R}$ indices (all data)

Absolute structure parameter
JF2862TW51 (JRJNpMeSi)

C25 H24 O Si

368.53

90(2) K

$0.71073 \AA$

Monoclinic

$\mathrm{P} 21$

$\mathrm{a}=7.9375(6) \AA \quad\left\langle=90^{\circ}\right.$.

$\mathrm{b}=20.4070(15) \AA \quad \Omega=104.2477(10)^{\circ}$.

$\mathrm{c}=12.5011(9) \AA \quad\left(c=90^{\circ}\right.$.

$1962.6(3) \AA^{3}$

4, 2

$1.247 \mathrm{Mg} / \mathrm{m}^{3}$

$0.131 \mathrm{~mm}^{-1}$

784

$0.755 \times 0.425 \times 0.282 \mathrm{~mm}^{3}$

Colorless Plate

Bruker APEX-II CCD

1.681 to $27.477^{\circ}$.

$-10<=\mathrm{h}<=10,-26<=\mathrm{k}<=26,-16<=\mathrm{k}<=16$

8983

8983

8671

$100.0 \%$

Semi-empirical from equivalents

0.9542 and 0.8639

SHELXT (Sheldrick, 2014)

SHELXL-2018/3 (Sheldrick, 2018) Full-matrix least-squares on $F^{2}$ $8983 / 8 / 588$

1.043

$\mathrm{R} 1=0.0333, \mathrm{wR} 2=0.0949$

$\mathrm{R} 1=0.0349, \mathrm{wR} 2=0.0964$

$0.06(7)$

Largest diff. peak and hole 0.231 and -0.204 e. $\AA^{-3}$ 


\section{References}

(1) Green, S. P.; Wheelhouse, K. M.; Payne, A. D.; Hallett, J. P.; Miller, P. W.; Bull, J. A. Thermal Stability and Explosive Hazard Assessment of Diazo Compounds and Diazo Transfer Reagents. Org. Process Res. Dev. 2020, 24 (1), 67-84. https://doi.org/10.1021/acs.oprd.9b00422.

(2) Yasutomi, Y.; Suematsu, H.; Katsuki, T. Iridium(III)-Catalyzed Enantioselective Si-H Bond Insertion and Formation of an Enantioenriched Silicon Center. J. Am. Chem. Soc. 2010, 132 (13), 4510-4511. https://doi.org/10.1021/ja100833h.

(3) Satoh, Y.; Igarashi, M.; Sato, K.; Shimada, S. Highly Selective Synthesis of Hydrosiloxanes by Au-Catalyzed Dehydrogenative Cross-Coupling Reaction of Silanols with Hydrosilanes. ACS Catal. 2017, 7 (3), 1836-1840. https://doi.org/10.1021/acscatal.6b03560.

(4) Lamb, K. N.; Squitieri, R. A.; Chintala, S. R.; Kwong, A. J.; Balmond, E. I.; Soldi, C.; Dmitrenko, O.; Castiñeira Reis, M.; Chung, R.; Addison, J. B.; Fettinger, J. C.; Hein, J. E.; Tantillo, D. J.; Fox, J. M.; Shaw, J. T. Synthesis of Benzodihydrofurans by Asymmetric C-H Insertion Reactions of Donor/Donor Rhodium Carbenes. Chem. - A Eur. J. 2017, 23 (49), 11843-11855. https://doi.org/10.1002/chem.201701630.

(5) Chen, J.; Chen, C.; Ji, C.; Lu, Z. Cobalt-Catalyzed Asymmetric Hydrogenation of 1,1Diarylethenes. Org. Lett. 2016, 18 (7), 1594-1597. https://doi.org/10.1021/acs.orglett.6b00453.

(6) Brown, E.; Chevalier, C.; Huet, F.; Le Grumelec, C.; Lézé, A.; Touet, J. Determination of the Enantiomeric Excesses of Chiral Acids by 19F NMR Studies of Their Esters Deriving from (R)(+)-2-(Trifluoromethyl)Benzhydrol. Tetrahedron: Asymmetry 1994, 5 (7), 1191-1194. https://doi.org/https://doi.org/10.1016/0957-4166(94)80154-1.

(7) Wu, C.; Hu, F.; Liu, Z.; Deng, G.; Ye, F.; Zhang, Y.; Wang, J. Cu(I)-Catalyzed Coupling of Diaryldiazomethanes with Terminal Alkynes: An Efficient Synthesis of Tri-Aryl-Substituted Allenes. Tetrahedron. 2015, pp 9196-9201. https://doi.org/10.1016/j.tet.2015.10.043.

(8) Cobo, A. A.; Armstrong, B. M.; Fettinger, J. C.; Franz, A. K. Catalytic Asymmetric Synthesis of Cyclopentene-Spirooxindoles Bearing Vinylsilanes Capable of Further Transformations. Org. Lett. 2019, 21 (20), 8196-8200. https://doi.org/10.1021/acs.orglett.9b02852.

(9) Karmel, C.; Chen, Z.; Hartwig, J. F. Iridium-Catalyzed Silylation of C-H Bonds in Unactivated Arenes: A Sterically Encumbered Phenanthroline Ligand Accelerates Catalysis. J. Am. Chem. Soc. 2019, 141 (17), 7063-7072. https://doi.org/10.1021/jacs.9b01972.

(10) Touge, T.; Nara, H.; Fujiwhara, M.; Kayaki, Y.; Ikariya, T. Efficient Access to Chiral Benzhydrols via Asymmetric Transfer Hydrogenation of Unsymmetrical Benzophenones with Bifunctional Oxo-Tethered Ruthenium Catalysts. J. Am. Chem. Soc. 2016, 138 (32), 10084-10087. https://doi.org/10.1021/jacs.6b05738.

(11) Corriu, R. J. P.; Guérin, C.; Moreau, J. J. E. Stereochemistry at Silicon. Topics in Stereochemistry. January 1, 1984, pp 43-198. https://doi.org/doi:10.1002/9780470147245.ch2.

(12) Oestreich, M. Chirality Transfer from Silicon to Carbon. Chem. - A Eur. J. 2006, 12 (1), 30-37. https://doi.org/10.1002/chem.200500782.

(13) Zhang, J.; Park, S.; Chang, S. Catalytic Access to Bridged Sila-N-Heterocycles from Piperidines via Cascade Sp3 and Sp2 C-Si Bond Formation. J. Am. Chem. Soc. 2018, 140 (41), 13209-13213. https://doi.org/10.1021/jacs.8b08733.

(14) Koga, S.; Ueki, S.; Shimada, M.; Ishii, R.; Kurihara, Y.; Yamanoi, Y.; Yuasa, J.; Kawai, T.; Uchida, T. A.; Iwamura, M.; Nozaki, K.; Nishihara, H. Access to Chiral Silicon Centers for Application to Circularly Polarized Luminescence Materials. J. Org. Chem. 2017, 82 (12), 61086117. https://doi.org/10.1021/acs.joc.7b00583.

(15) Yasutomi, Y.; Suematsu, H.; Katsuki, T. Iridium(III)-Catalyzed Enantioselective Si-H Bond Insertion and Formation of an Enantioenriched Silicon Center. J. Am. Chem. Soc. 2010, 132 (13), 4510-4511. https://doi.org/10.1021/ja100833h.

(16) Lee, M.; Ren, Z.; Musaev, D. G.; Davies, H. M. L. Rhodium-Stabilized Diarylcarbenes Behaving as Donor/Acceptor Carbenes. ACS Catal. 2020, 6240-6247.

https://doi.org/10.1021/acscatal.0c01131. 
(17) Lasri, J.; Aly, M. M.; Eltayeb, N. E.; Babgi, B. A. Synthesis of Symmetrical and Asymmetrical Azines from Hydrazones and/or Ferrocenecarboxaldehyde as Potential Antimicrobial-Antitumor Agents. J. Mol. Struct. 2018, 1164, 1-8. https://doi.org/https://doi.org/10.1016/j.molstruc.2018.03.030.

(18) Momma, K.; Izumi, F. \{ lit VESTA3\} for Three-Dimensional Visualization of Crystal, Volumetric and Morphology Data. J. Appl. Crystallogr. 2011, 44 (6), 1272-1276. https://doi.org/10.1107/S0021889811038970.

(19) Bruker (2013) APEXII (Version 2014.9-1) and (2016) SAINT (Version 8.37a). Bruker AXS Inc., Madison, Wisconsin, USA.

(20) Sheldrick, G.M., CELLNOW, Twin matrix determination program, Universität Göttingen: Göttingen, Germany, Version 2008/3.

(21) An Empirical Correction for Absorption Anisotropy, Blessing, R. H. (1995). Acta Cryst., A51, 33-38.

(22) Sheldrick, G.M., TWINABS Version 2012/1'An Empirical Correction for Absorption Anisotropy applied to Twinned crystals'. Universität Göttingen: Göttingen, Germany.

(23) Sheldrick, G.M., (2002). SHELXTL. Version 6.1. Siemens Analytical X-ray Instruments Inc., Madison, Wisconsin, USA.

(24) Sheldrick, G. M., (2014). SHELXT. Universität Göttingen: Göttingen, Germany.

(25) Sheldrick, G. M., SHELXL2017/3 structure refined program - unpublished.

(26) On Enantiomorph-Polarity Estimation, Flack, H.D. (1983). Acta Cryst., A39, 876-881. 
IX. Copies of NMR Spectra

$$
\overbrace{\substack{\mathrm{Si}^{\prime}{ }^{\mathrm{D}} \mathrm{d}_{2} \\ \mathrm{CH}_{3}}}^{\mathrm{CH}_{3}}
$$

${ }^{1} \mathrm{H}, 600 \mathrm{MHz}, \mathrm{CDCl}_{3}$

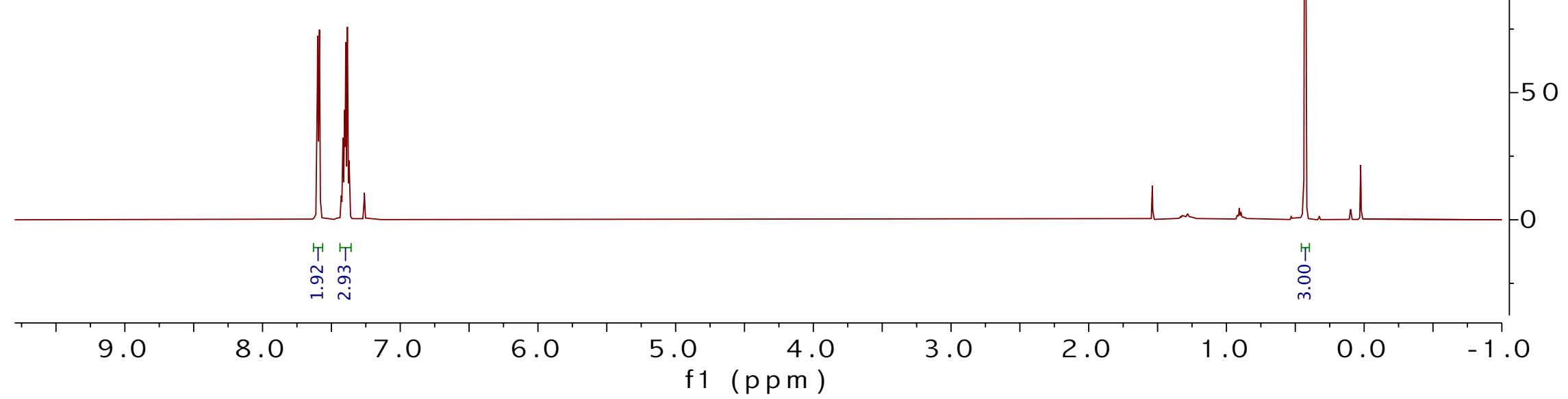




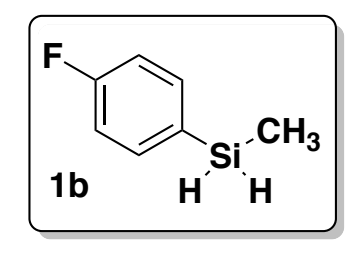

$\rightarrow \infty$

نَ

$\stackrel{\infty}{m} \hat{m} m$

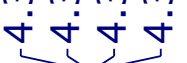

1000

${ }^{1} \mathrm{H}, 600 \mathrm{MHz}, \mathrm{C}_{6} \mathrm{D}_{6}$

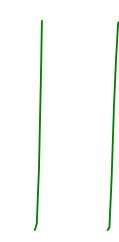

900

800

700

600

500

400

300

200

100

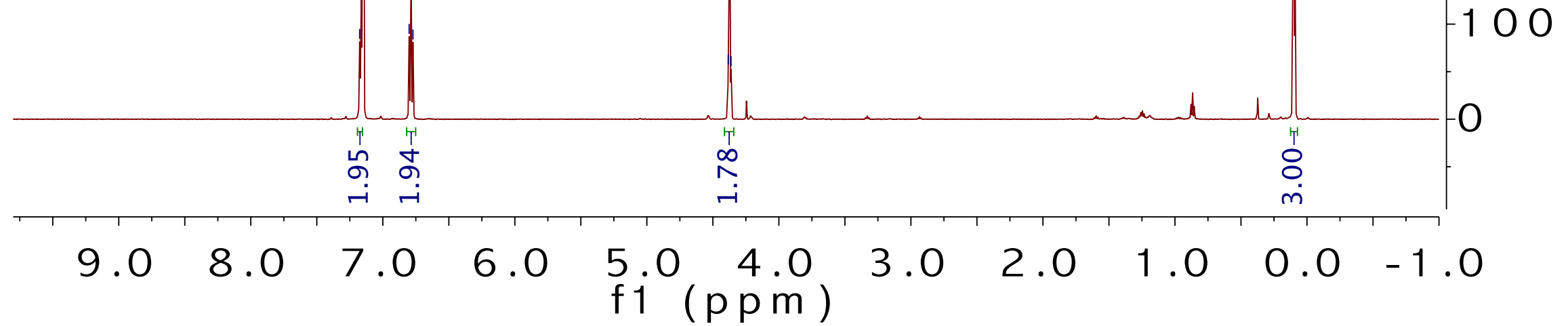




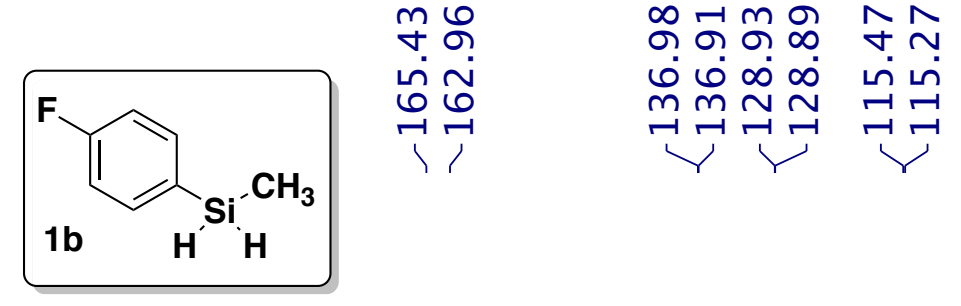

${ }^{13} \mathrm{C}, 100 \mathrm{MHz}, \mathrm{CDCl}_{3}$

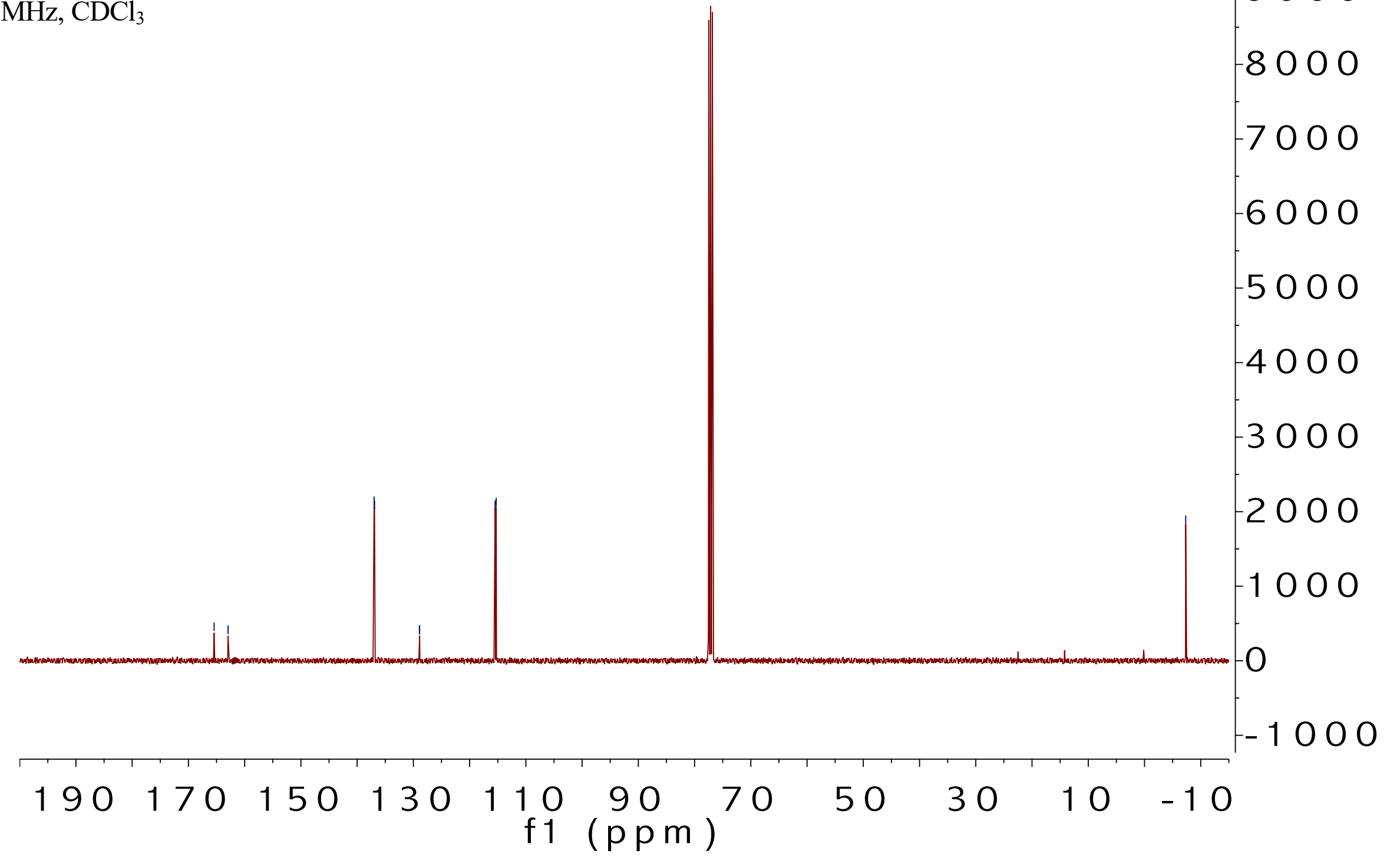




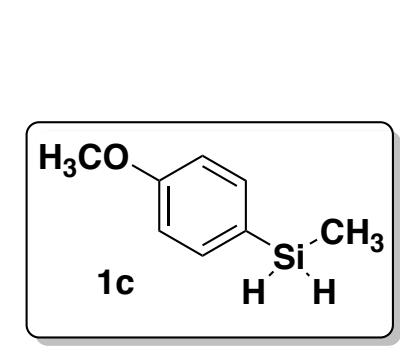

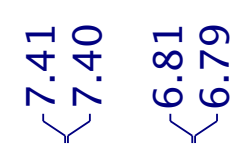

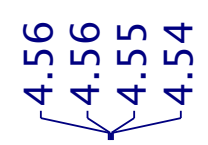

${ }^{1} \mathrm{H}, 600 \mathrm{MHz}, \mathrm{C}_{6} \mathrm{D}_{6}$

260

220

180

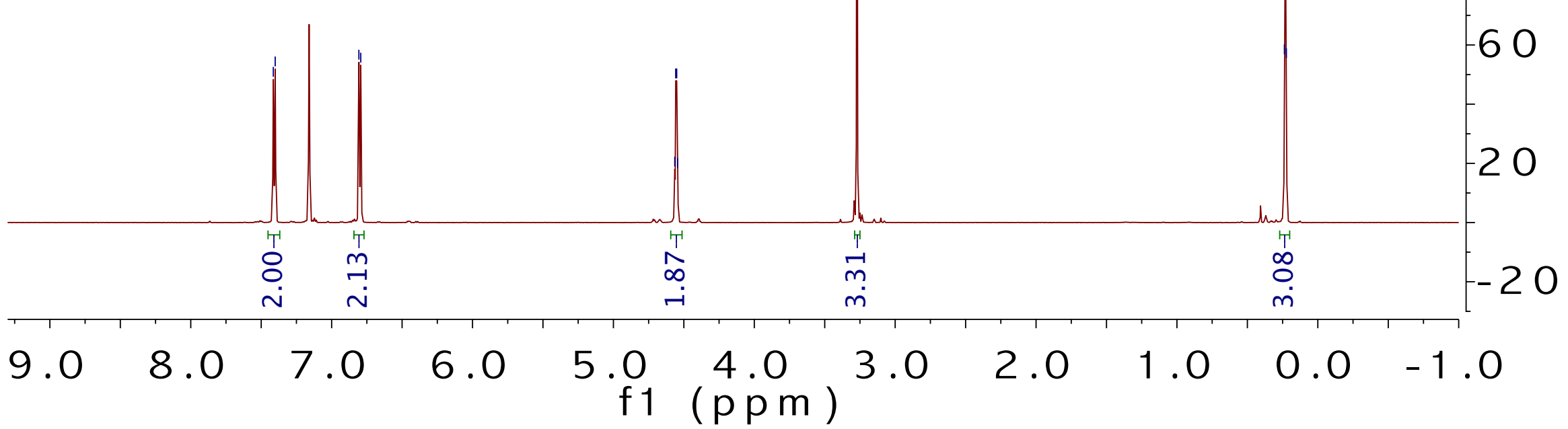




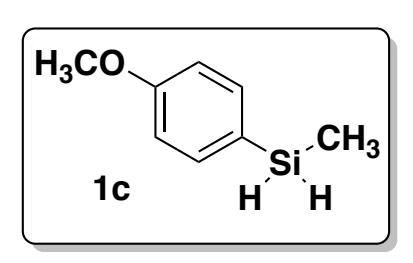

${ }^{13} \mathrm{C}, 100 \mathrm{MHz}, \mathrm{CDCl}_{3}$

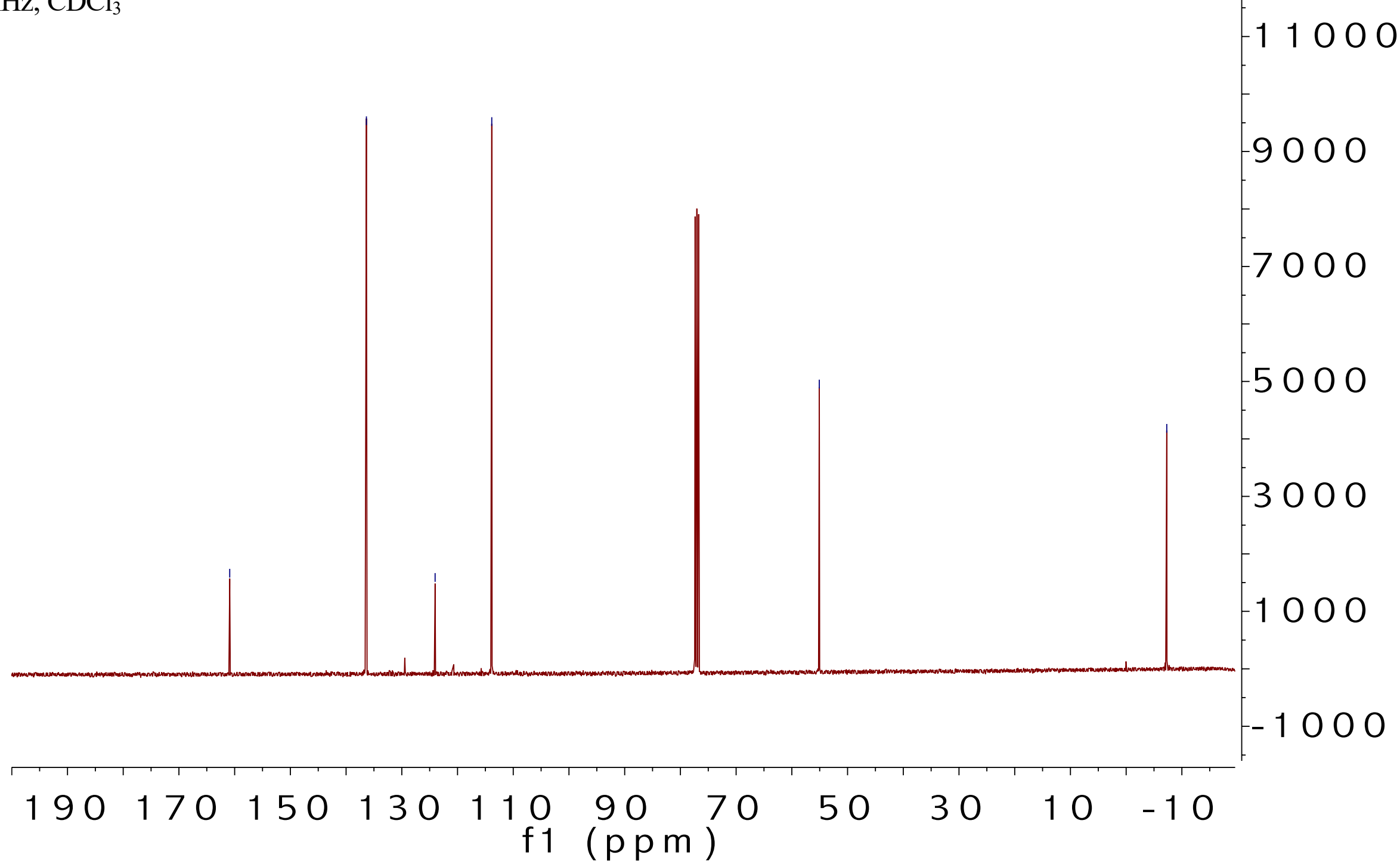




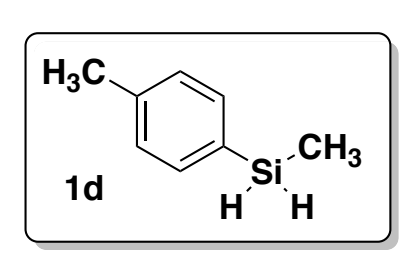

${ }^{1} \mathrm{H}, 600 \mathrm{MHz}, \mathrm{C}_{6} \mathrm{D}_{6}$

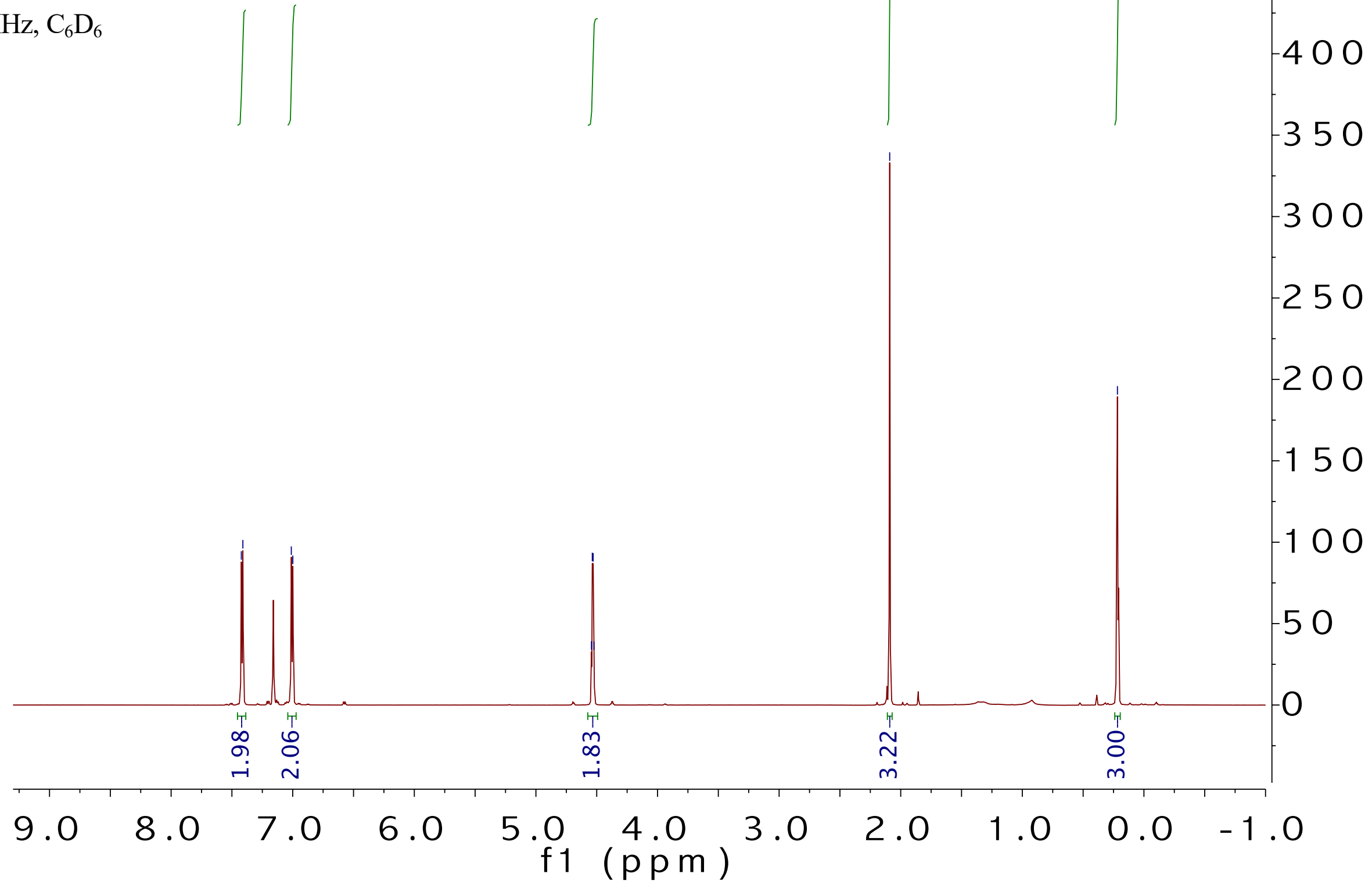



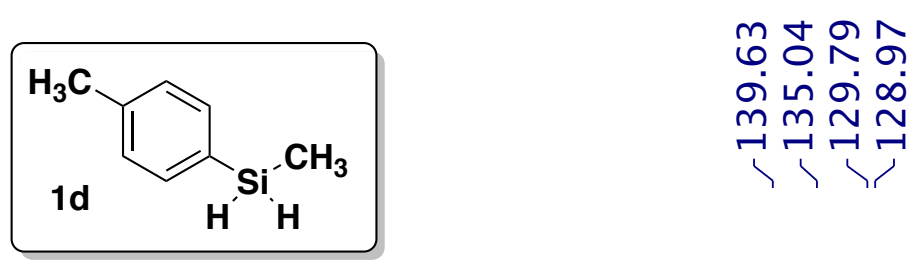

${ }^{13} \mathrm{C}, 100 \mathrm{MHz}, \mathrm{CDCl}_{3}$

$-2500$

1500

500 


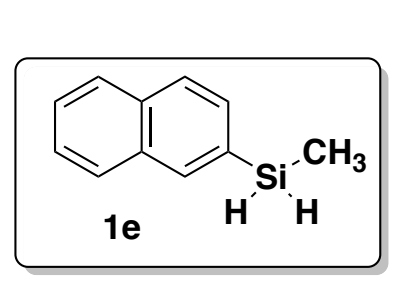

${ }^{1} \mathrm{H}, 600 \mathrm{MHz}, \mathrm{C}_{6} \mathrm{D}_{6}$

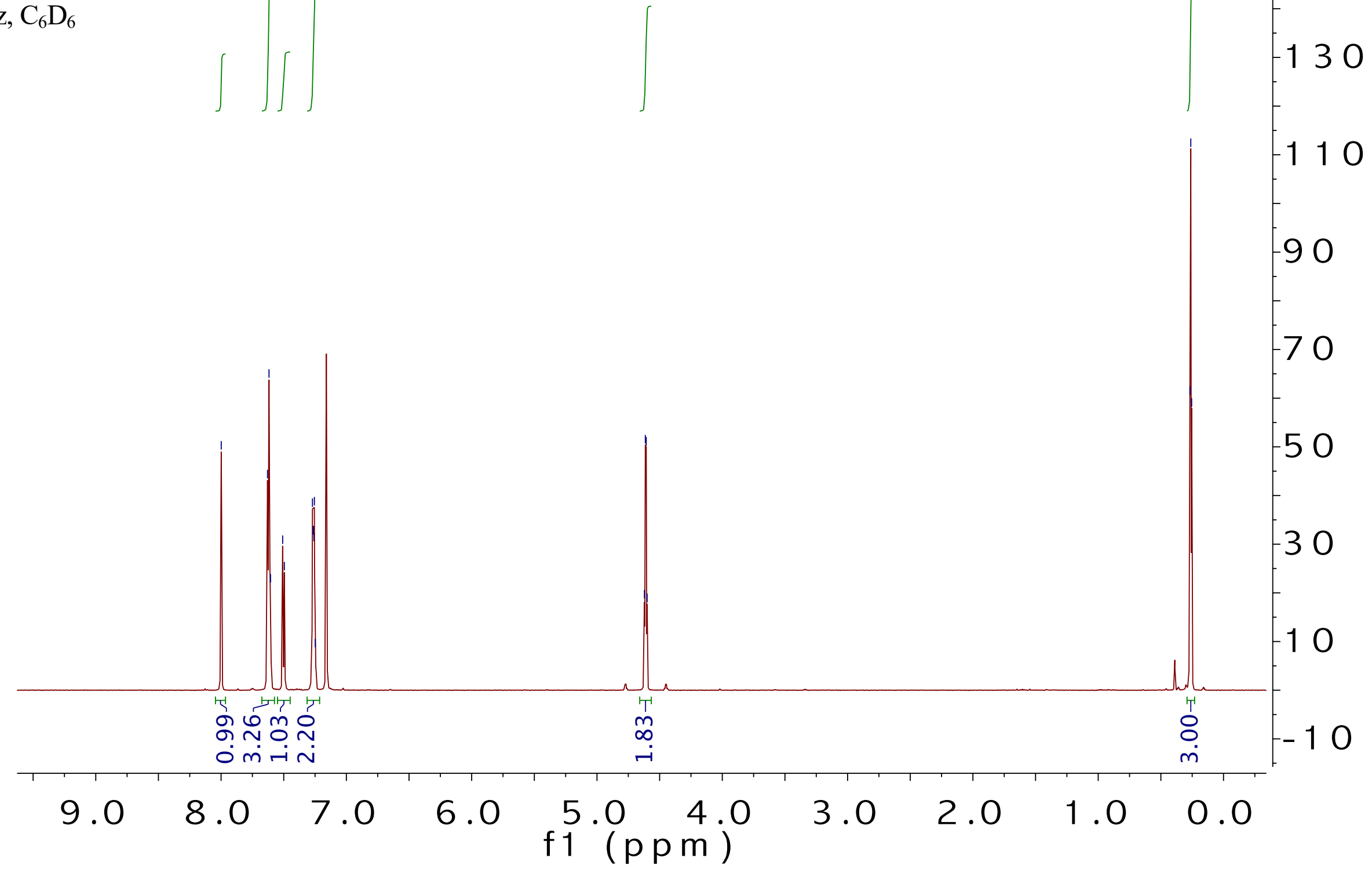




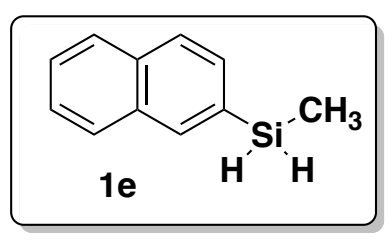




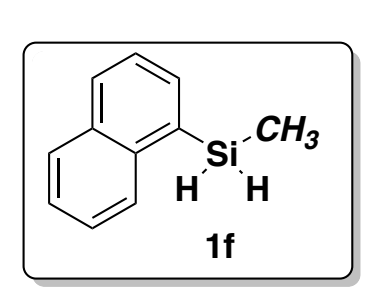

คㅇ

${ }^{1} \mathrm{H}, 600 \mathrm{MHz}, \mathrm{CDCl}_{3}$

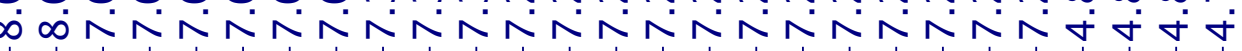

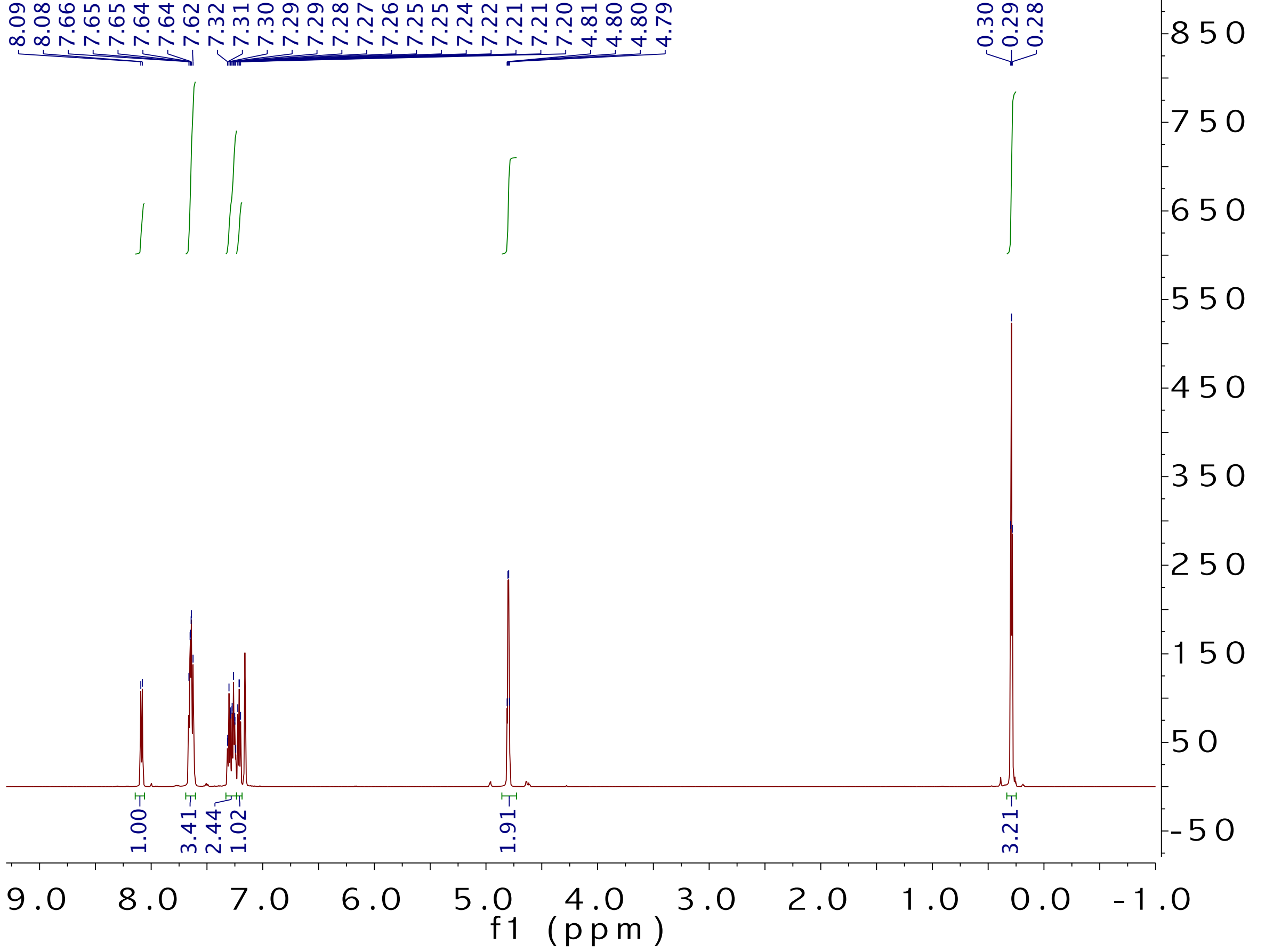




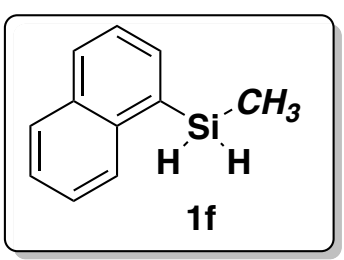

${ }^{13} \mathrm{C}, 151 \mathrm{MHz}, \mathrm{CDCl}_{3}$ 


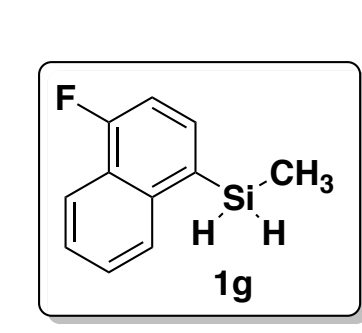

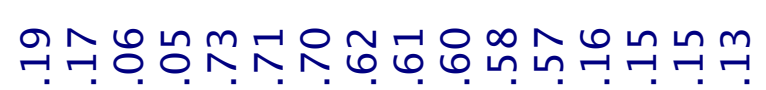

${ }^{1} \mathrm{H}, 400 \mathrm{MHz}, \mathrm{CDCl}_{3}$
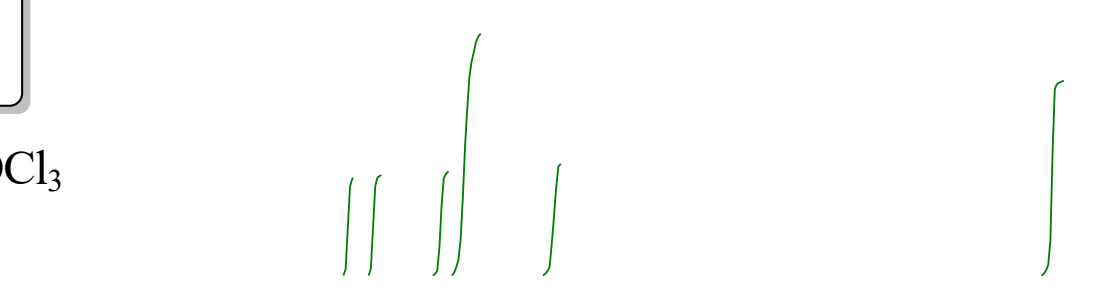

$-450$

$-400$

350

300

250

200

150

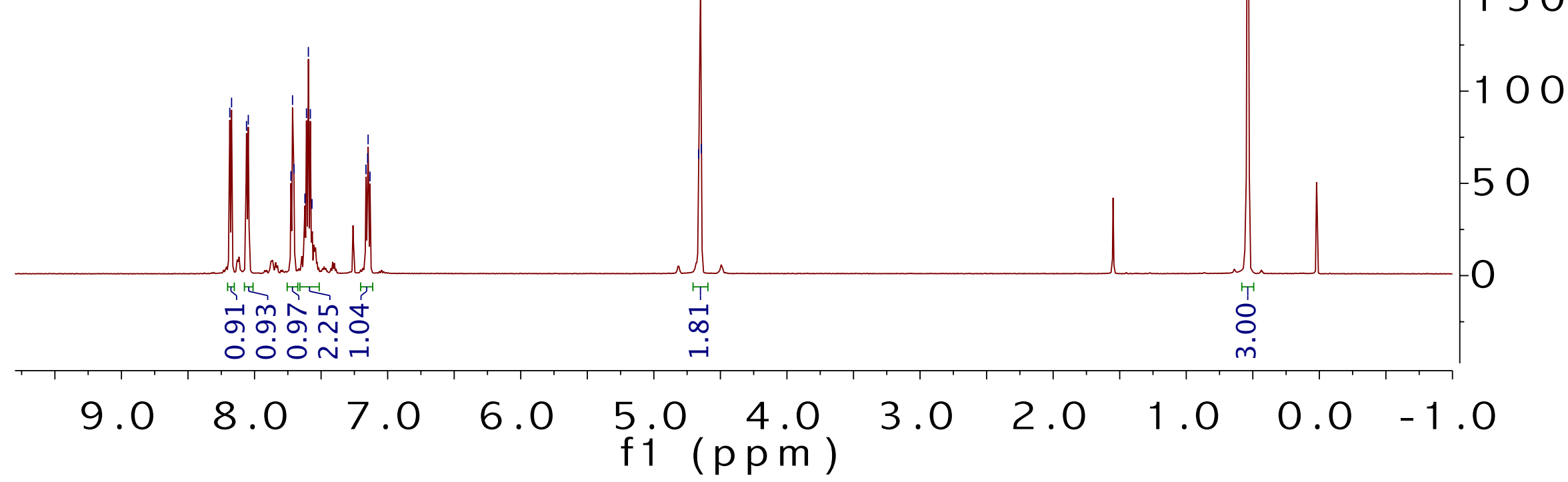




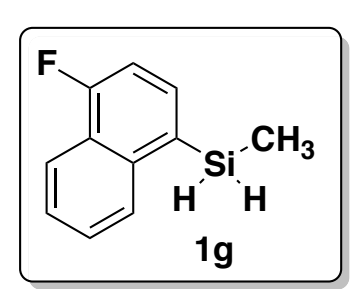



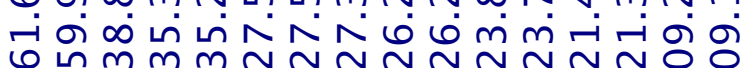

$\underbrace{0}$

${ }^{13} \mathrm{C}, 151 \mathrm{MHz}, \mathrm{CDCl}_{3}$

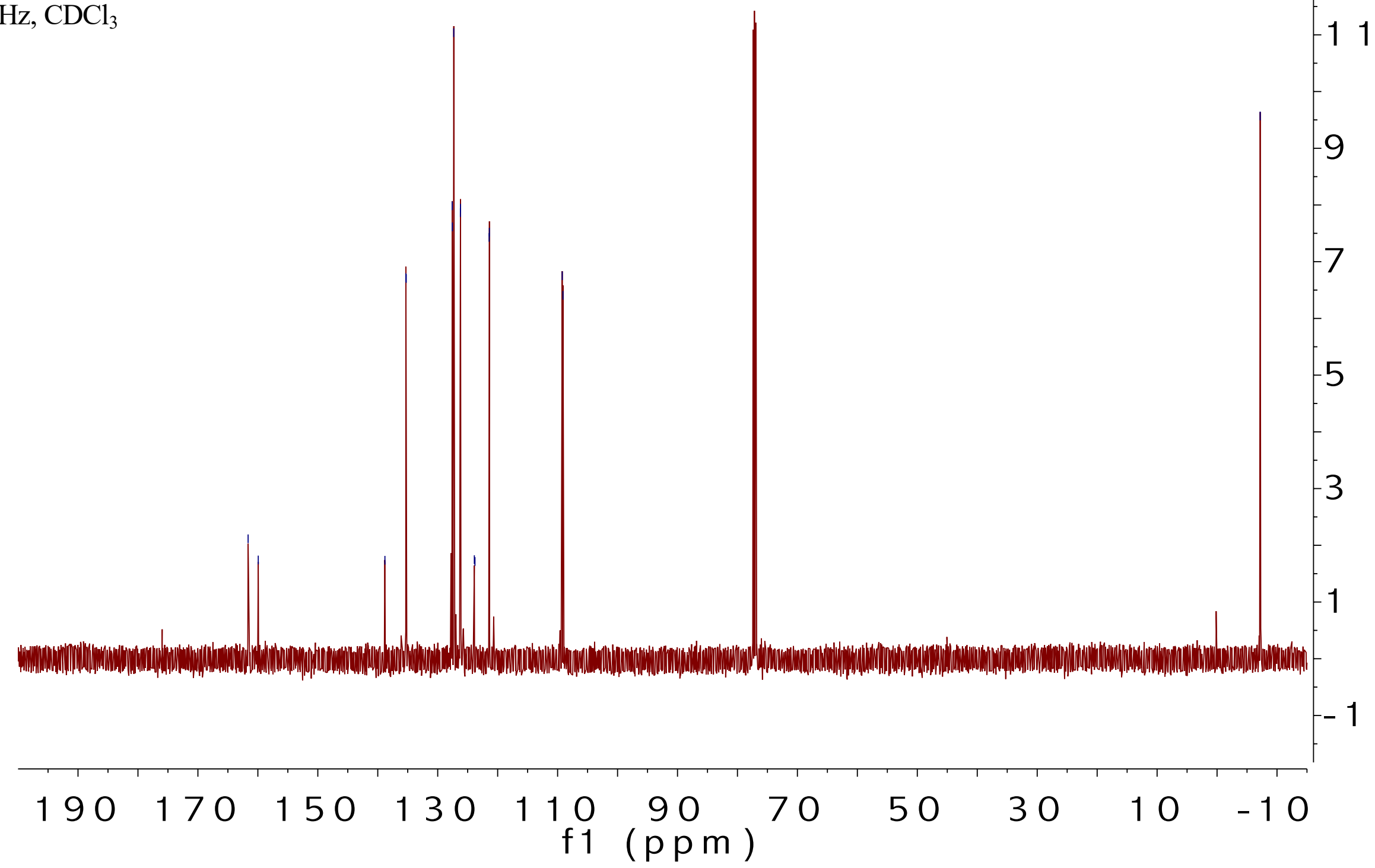




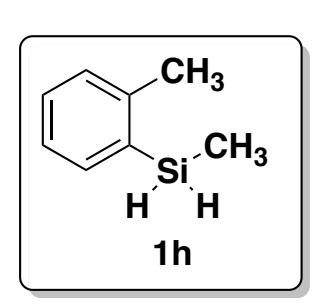

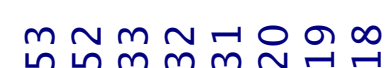

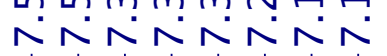

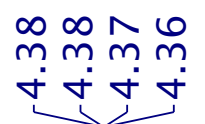

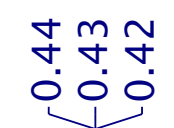

12200

${ }^{1} \mathrm{H}, 600 \mathrm{MHz}, \mathrm{CDCl}_{3}$

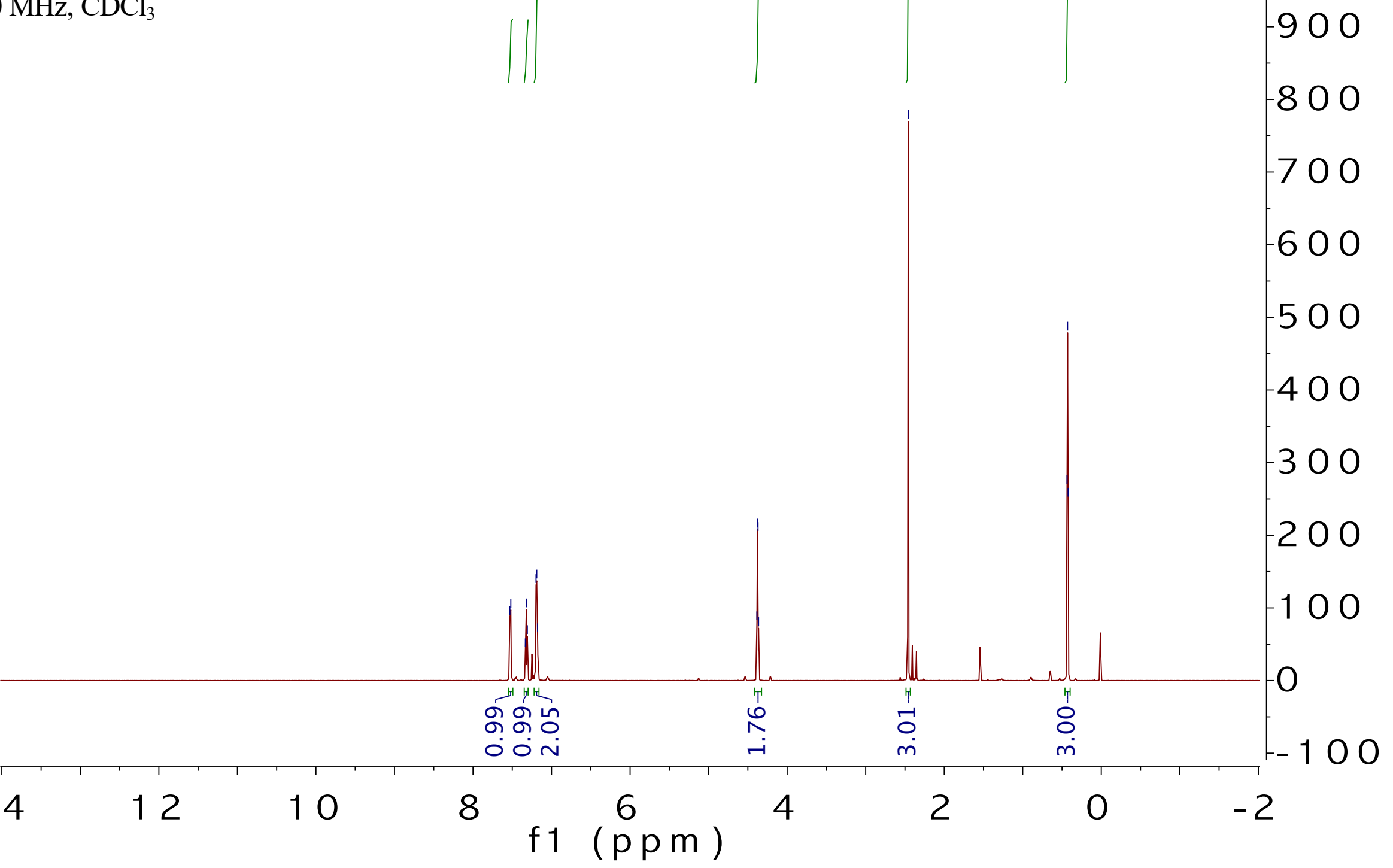




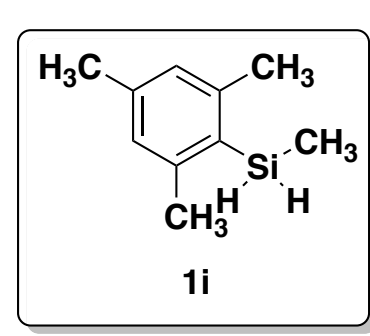

${ }^{1} \mathrm{H}, 400 \mathrm{MHz}, \mathrm{CDCl}_{3}$

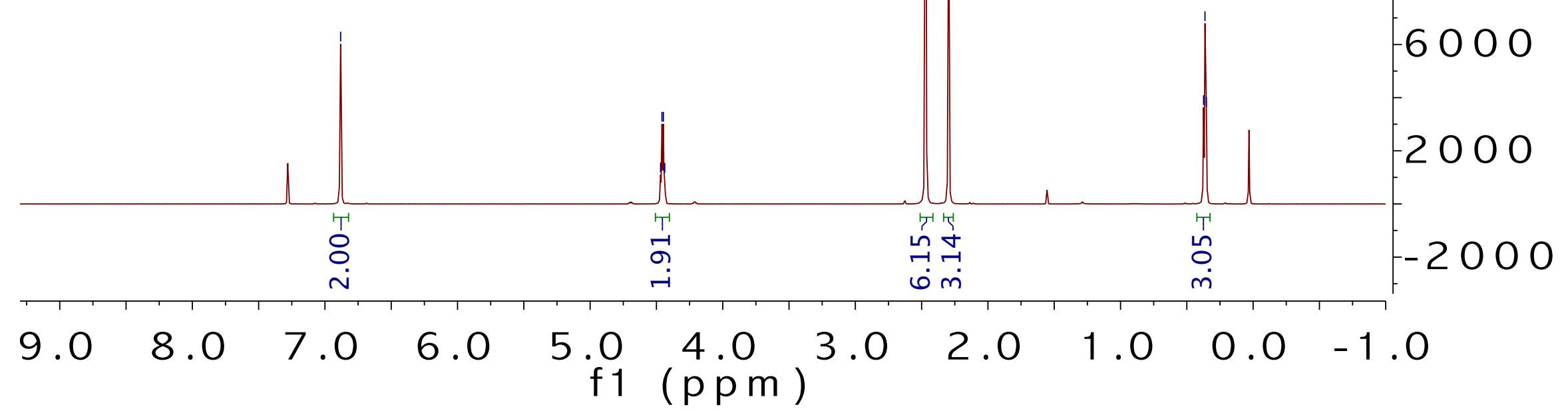




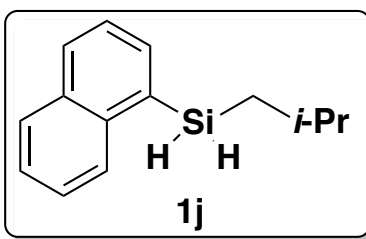

${ }^{1} \mathrm{H}, 600 \mathrm{MHz}, \mathrm{C}_{6} \mathrm{D}_{6}$
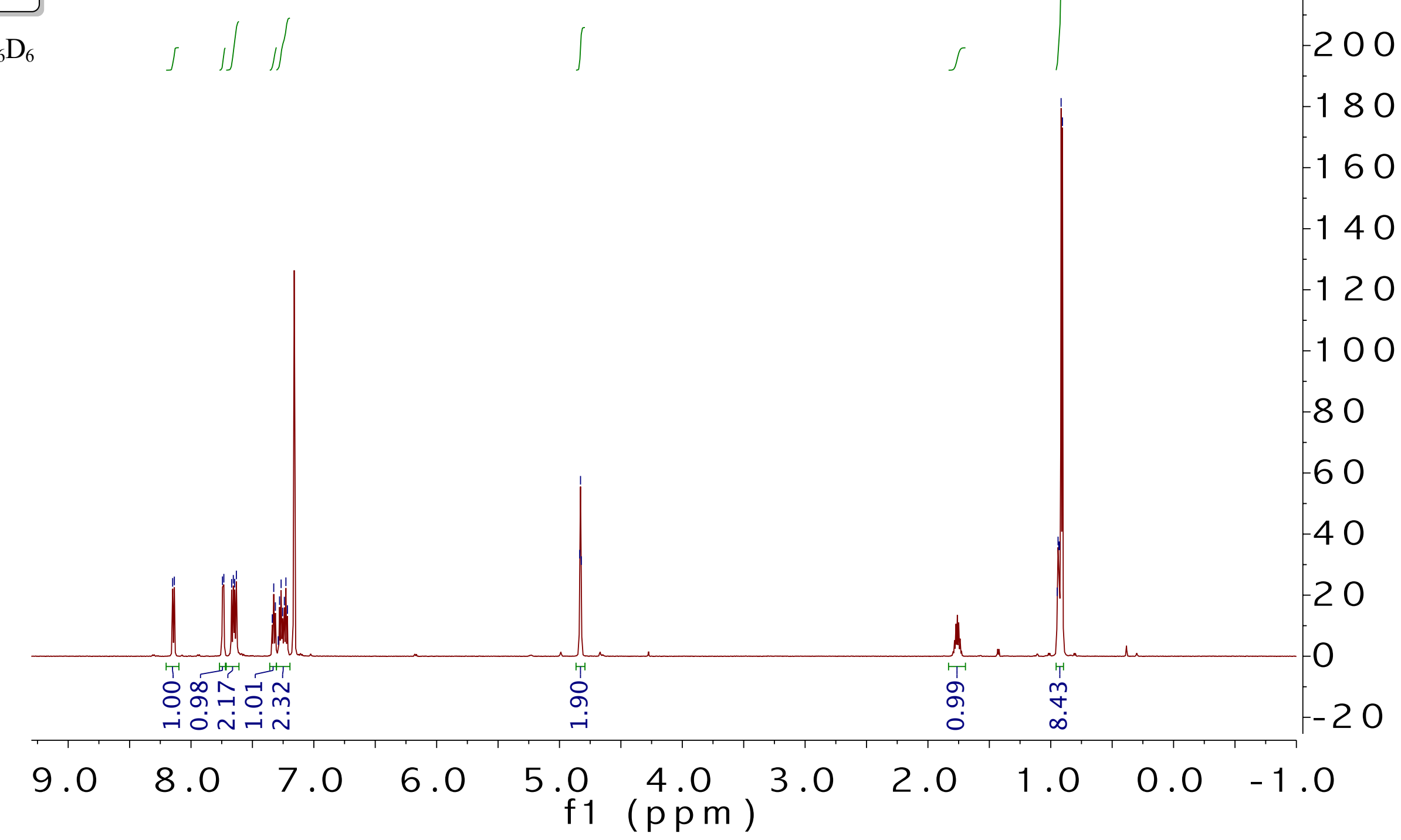
${ }^{13} \mathrm{C}, 151 \mathrm{MHz}, \mathrm{CDCl}_{3}$

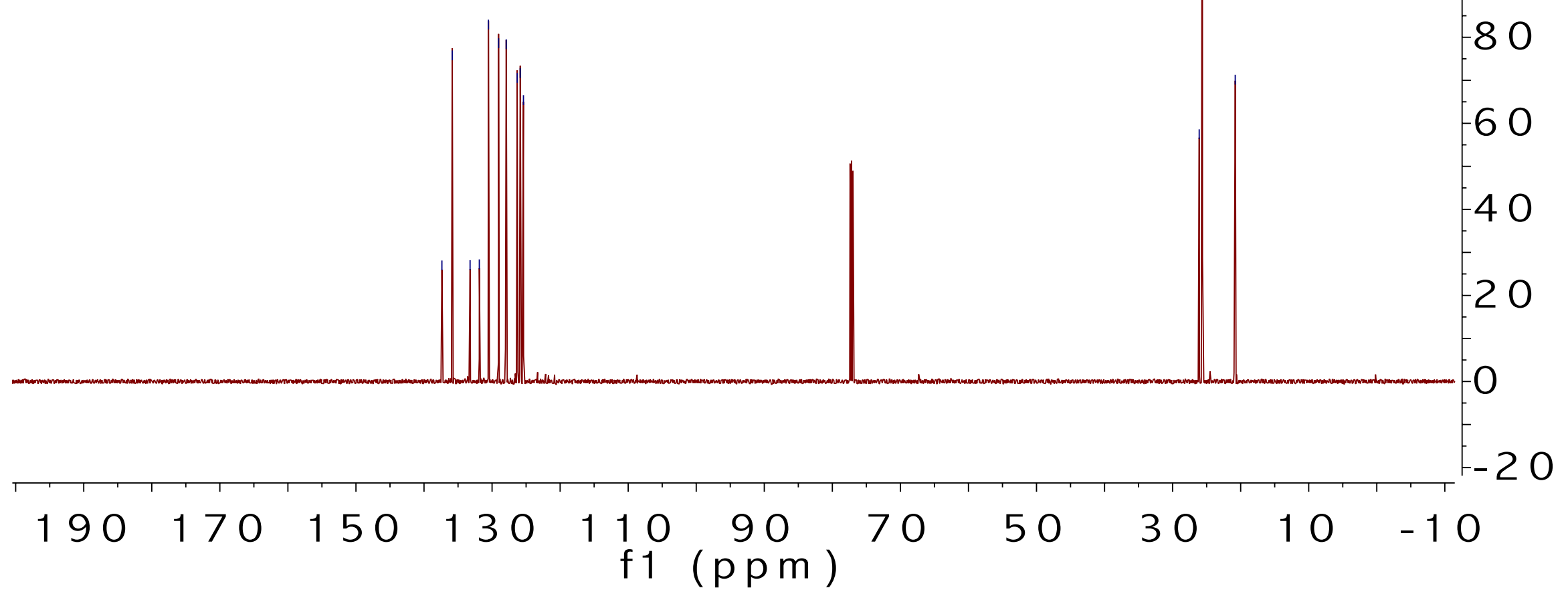




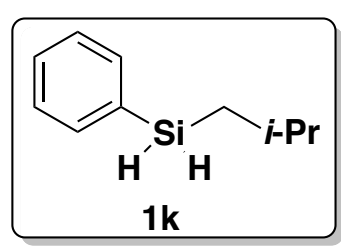

N

${ }^{1} \mathrm{H}, 600 \mathrm{MHz}, \mathrm{CDCl}_{3}$

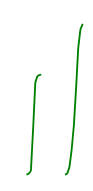

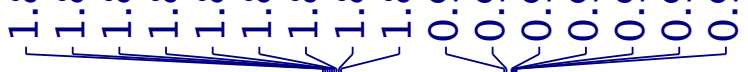

1500

1300

1100

900

700

500

300

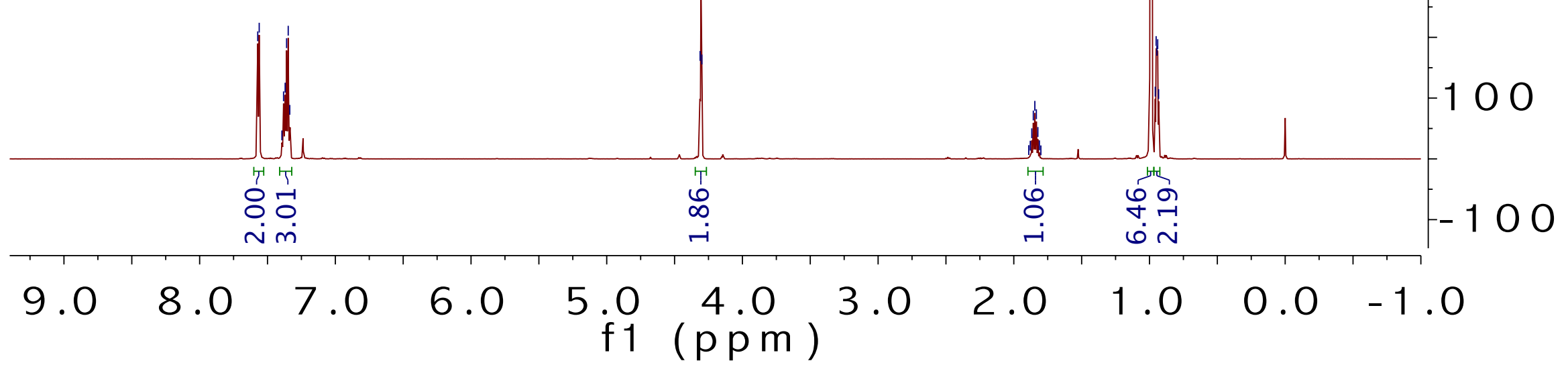




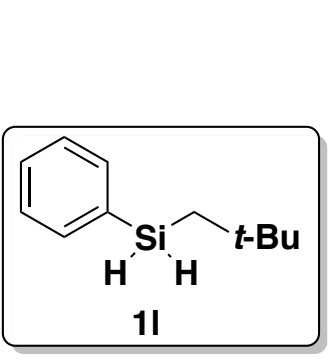

${ }^{1} \mathrm{H}, 600 \mathrm{MHz}, \mathrm{CDCl}_{3}$

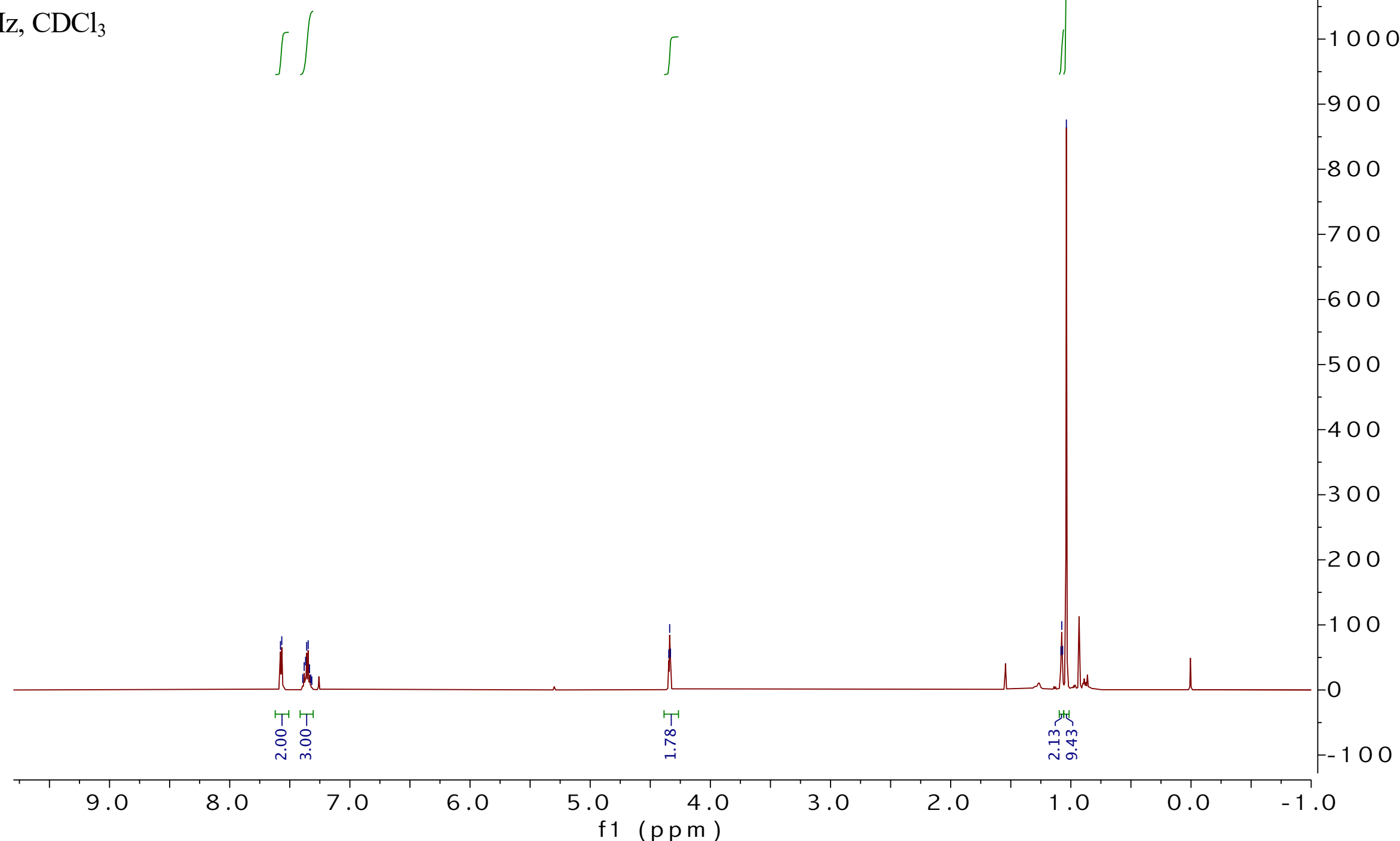




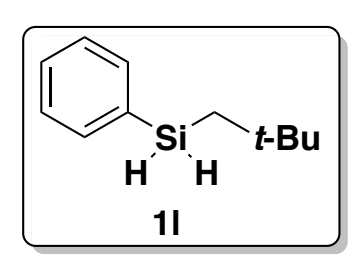

${ }^{13} \mathrm{C}, 151 \mathrm{MHz}, \mathrm{CDCl}_{3}$

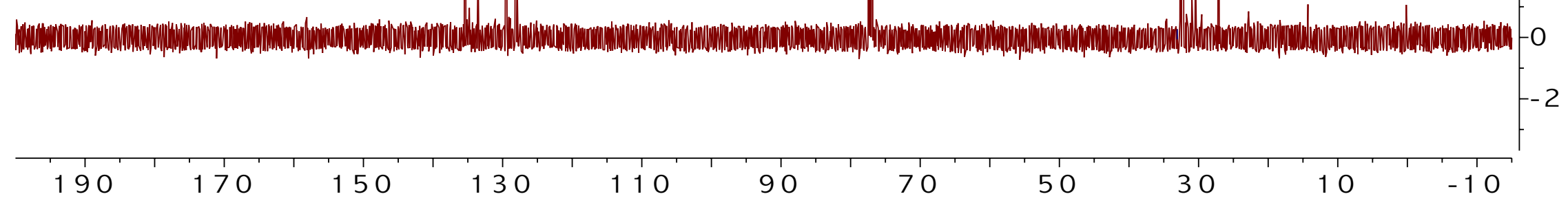




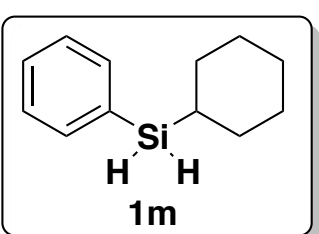

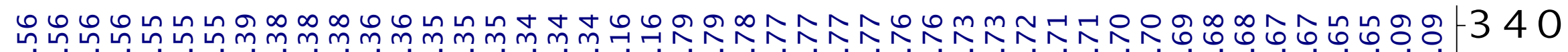

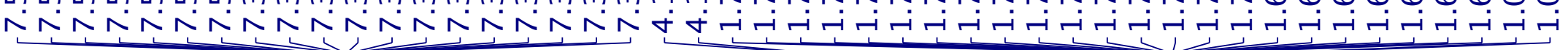

${ }^{1} \mathrm{H}, 600 \mathrm{MHz}, \mathrm{CDCl}_{3}$

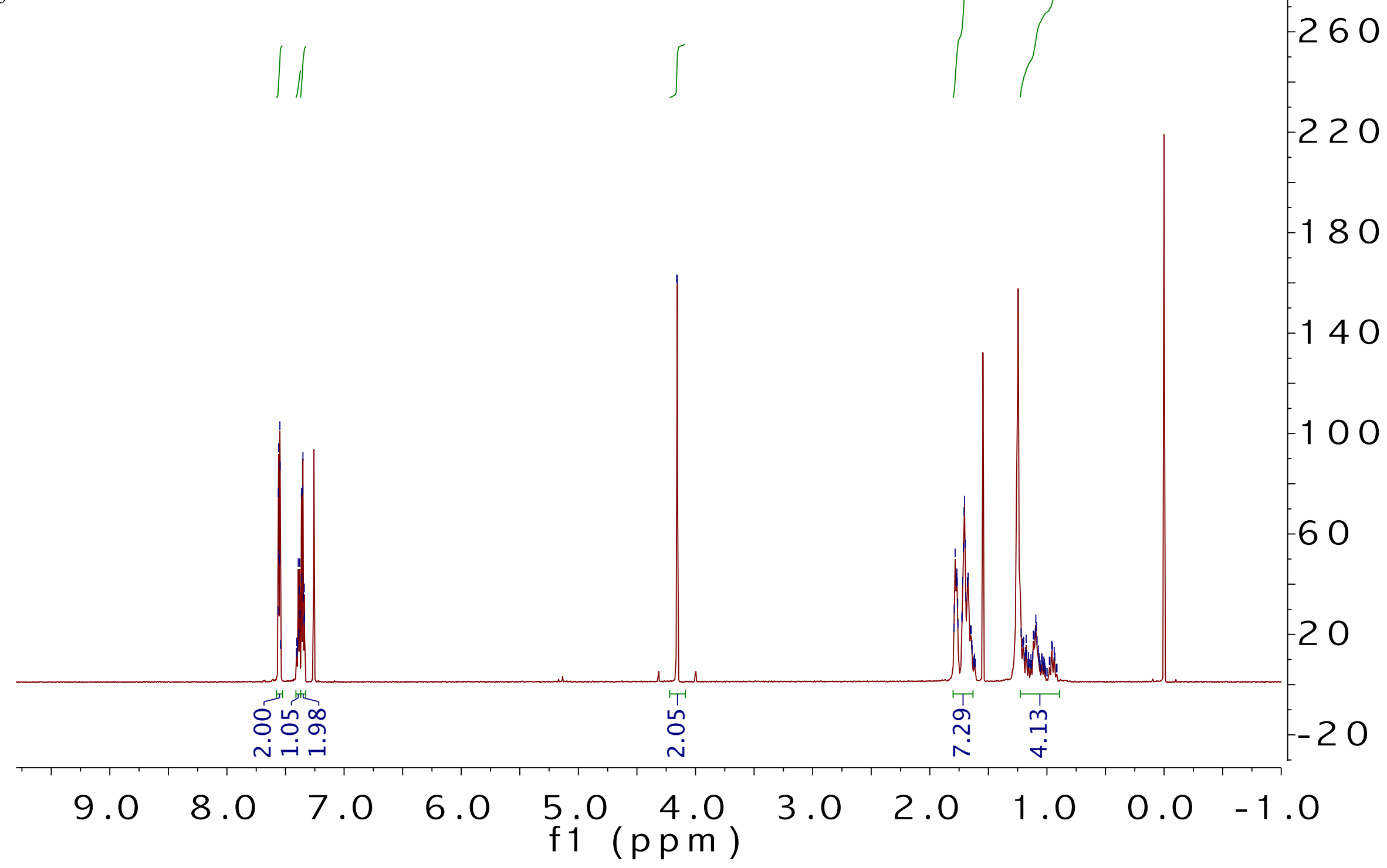




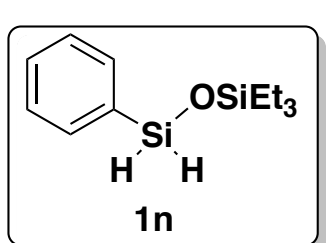

${ }^{1} \mathrm{H}, 400 \mathrm{MHz}, \mathrm{CDCl}_{3}$

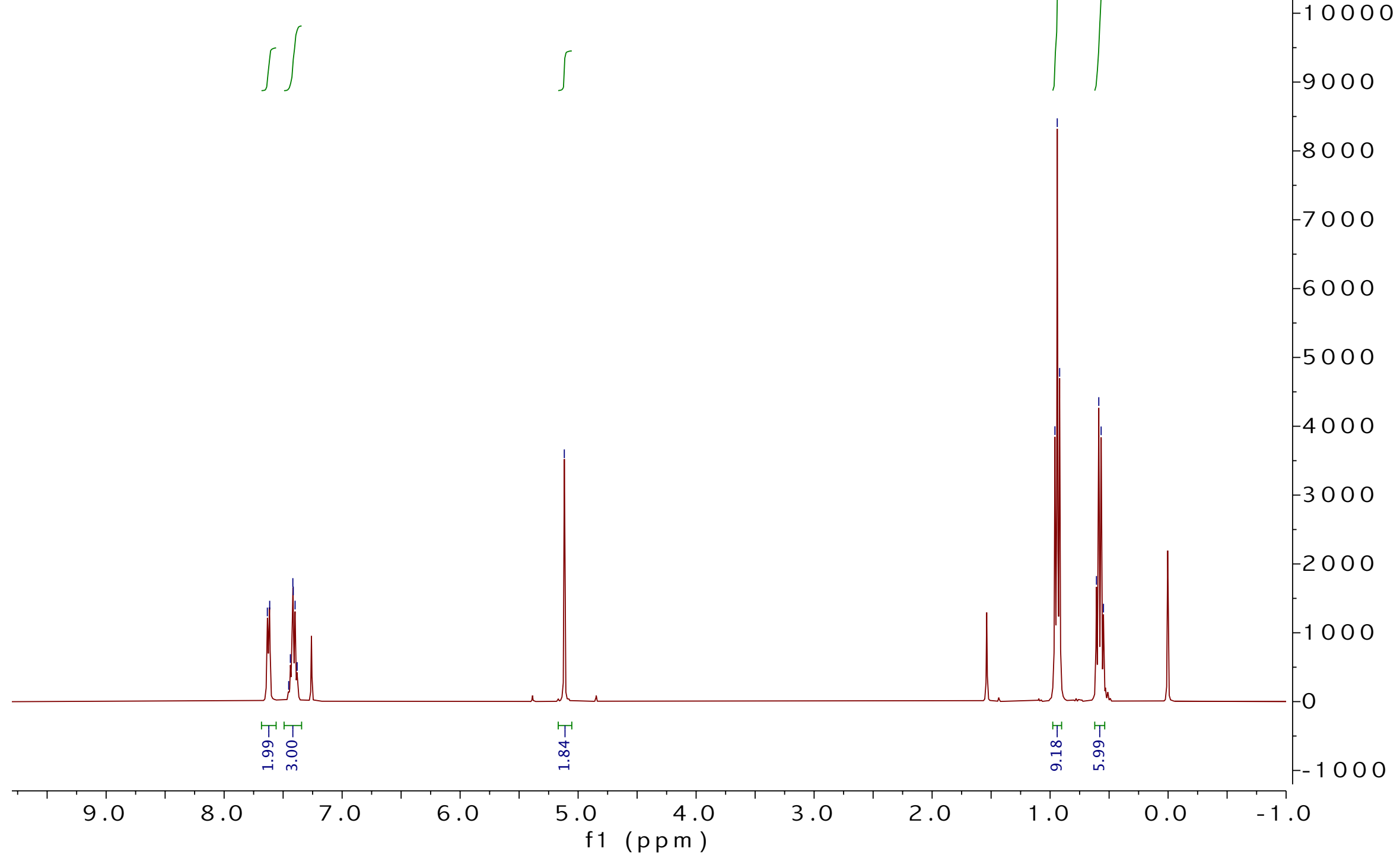




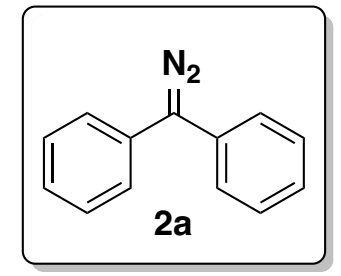

10000

$-9000$

${ }^{1} \mathrm{H}, 400 \mathrm{MHz}, \mathrm{CDCl}_{3}$

8000

$-7000$

$-6000$

$-5000$

$-4000$

$-3000$

$-2000$

$-1000$

7.0 


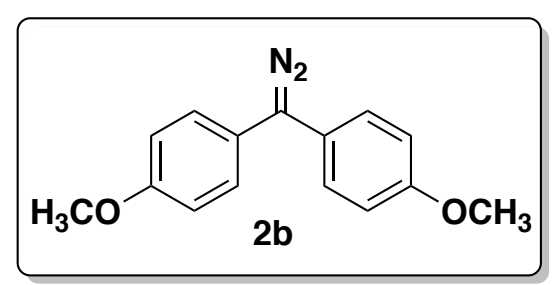

${ }^{1} \mathrm{H}, 600 \mathrm{MHz}, \mathrm{CDCl}_{3}$

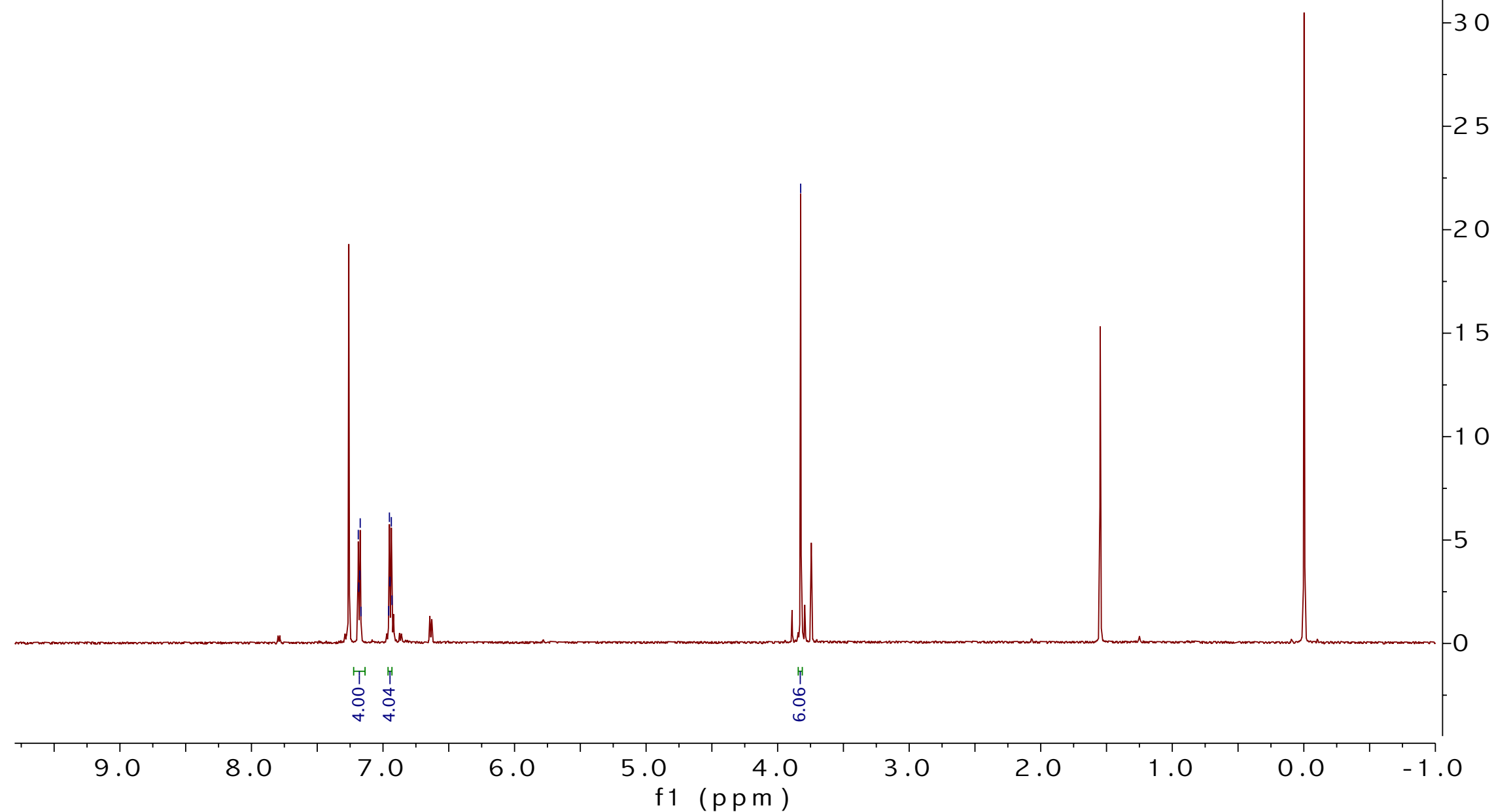




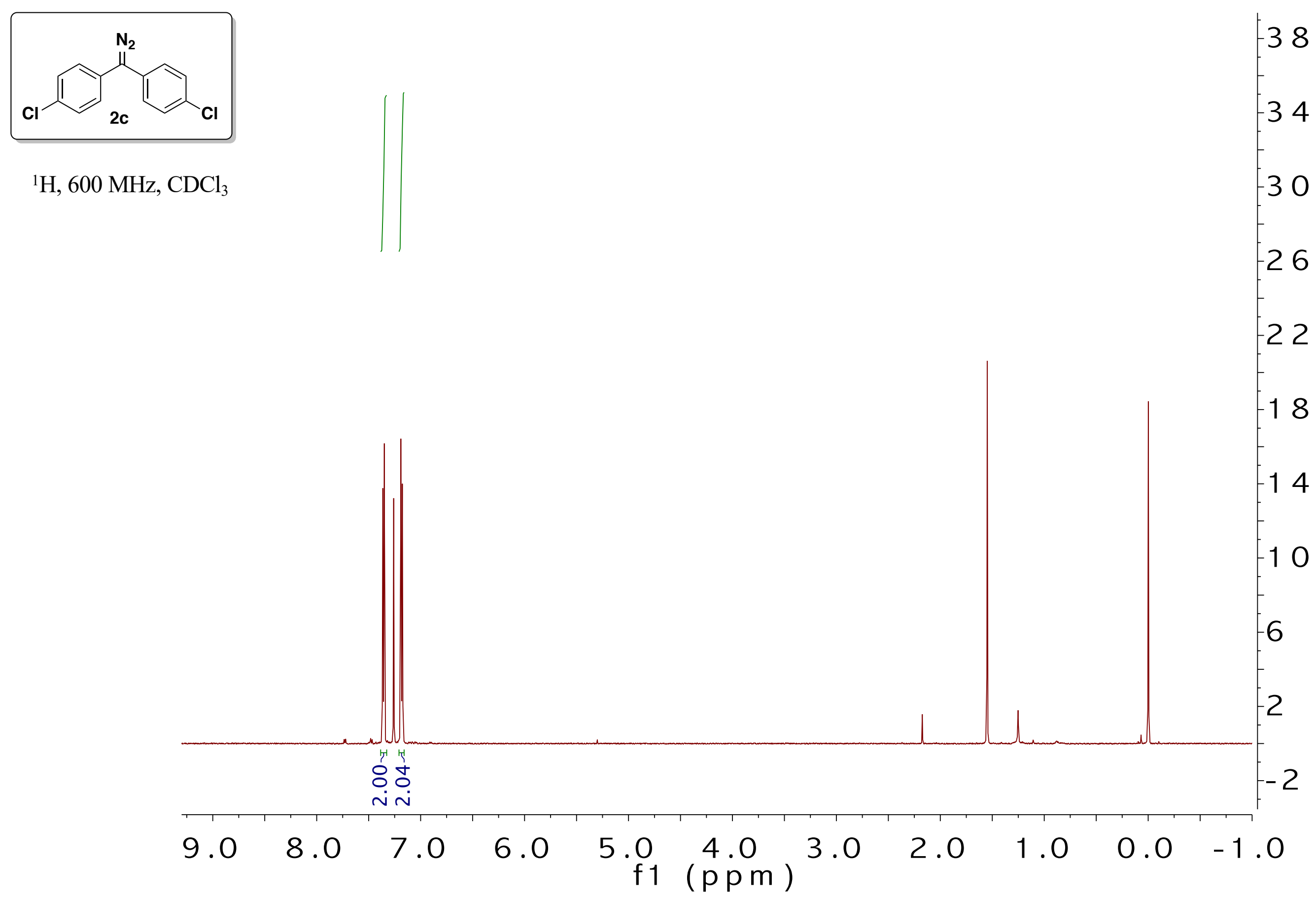




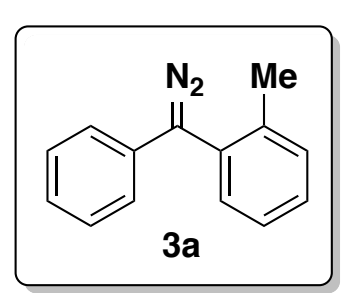

${ }^{1} \mathrm{H}, 600 \mathrm{MHz}, \mathrm{CDCl}_{3}$

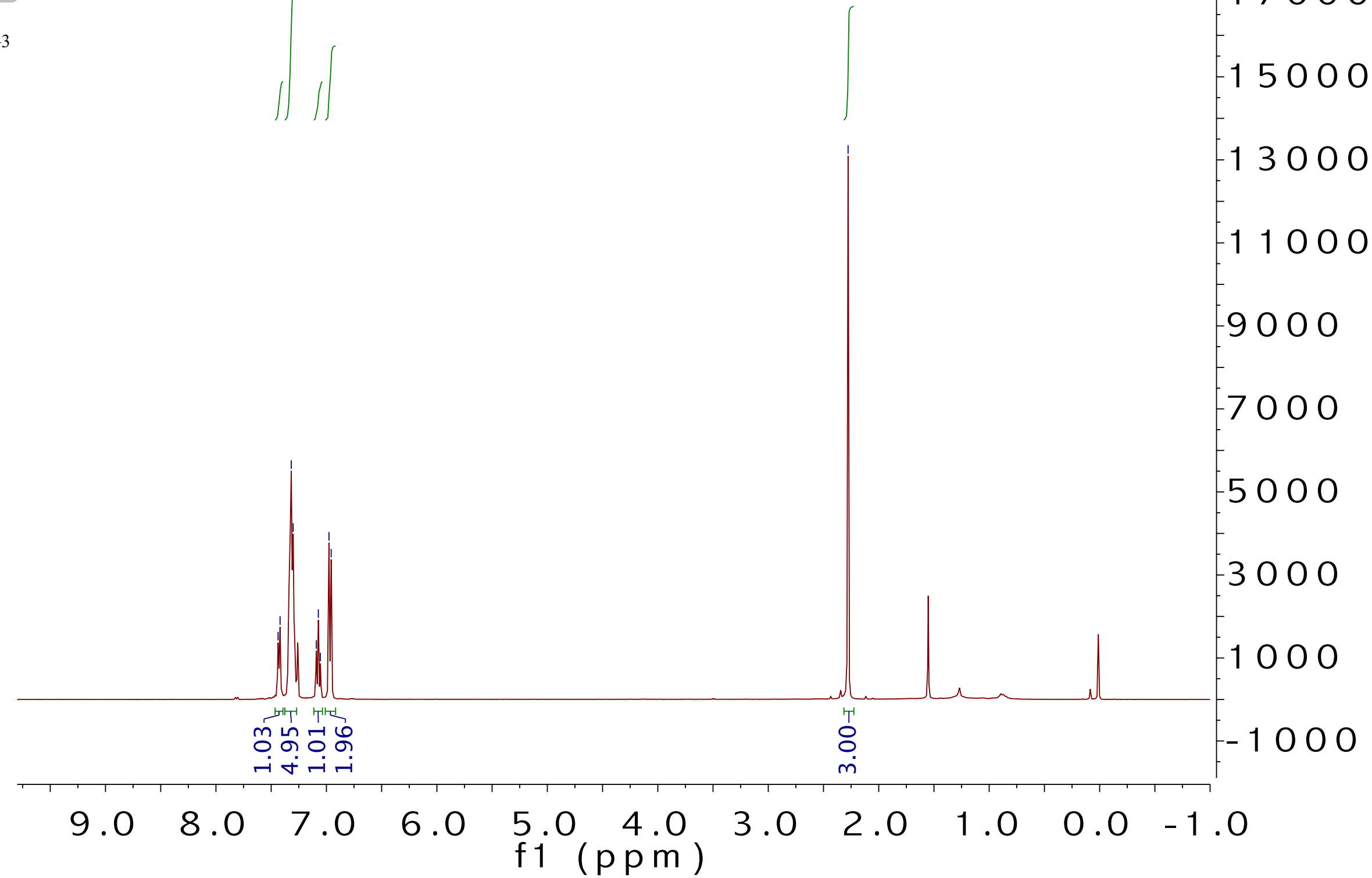




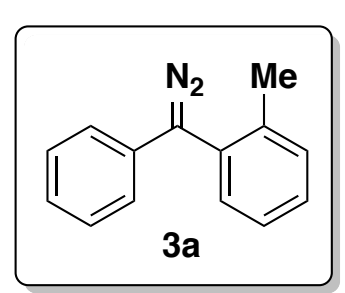

${ }^{13} \mathrm{C}, 151 \mathrm{MHz}, \mathrm{CDCl}_{3}$

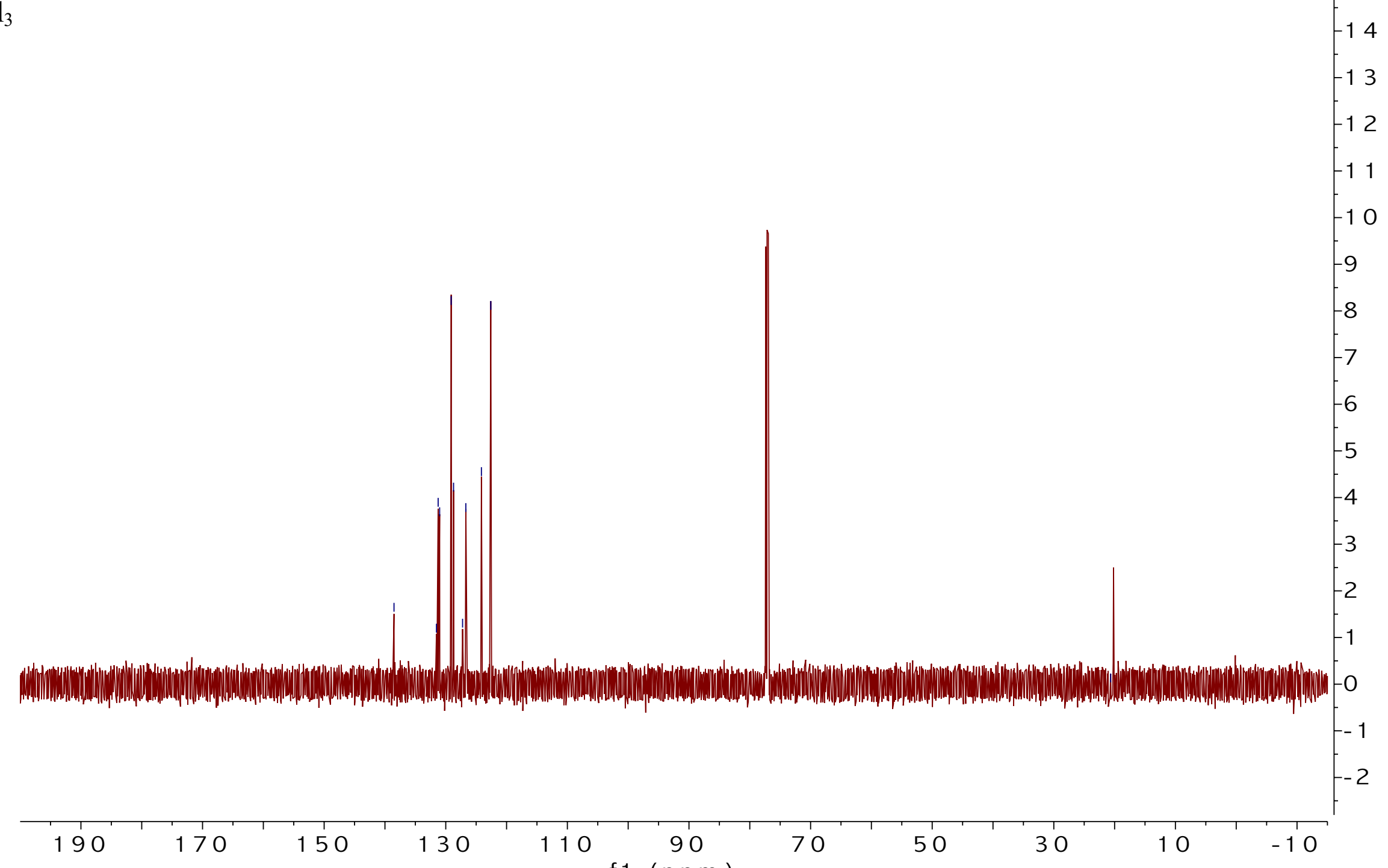




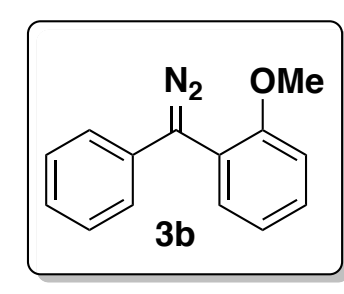

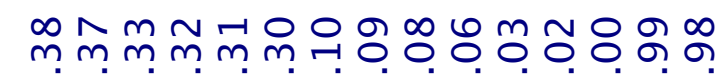

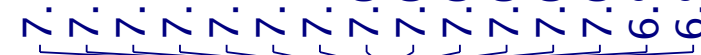

${ }^{1} \mathrm{H}, 600 \mathrm{MHz}, \mathrm{CDCl}_{3}$

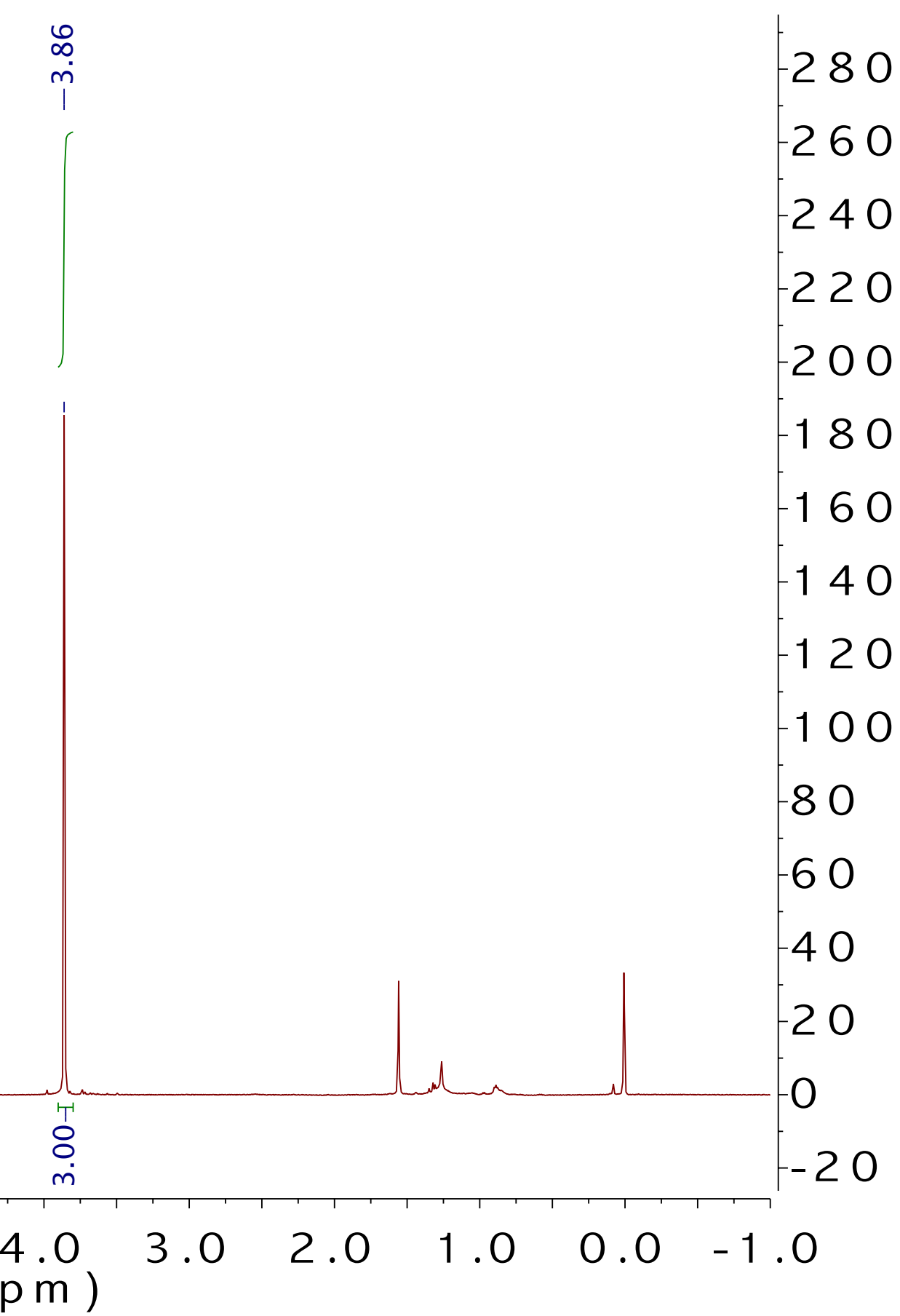




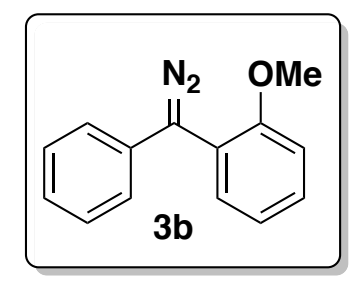

몬

㭊

กิ

10000

$-9000$

8000

$-7000$

$-6000$

5000

4000

3000

2000

1000

0

$-1000$

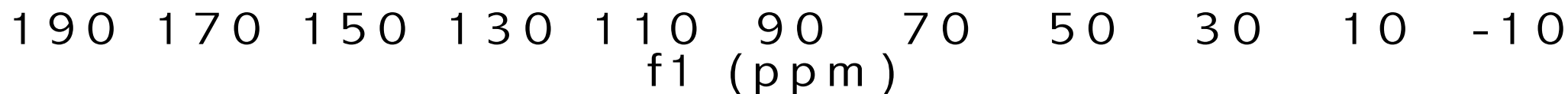




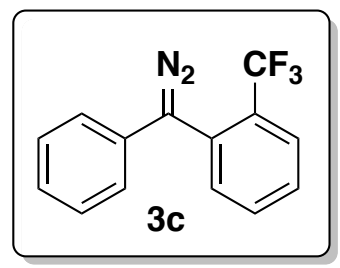

${ }^{1} \mathrm{H}, 400 \mathrm{MHz}, \mathrm{CDCl}_{3}$

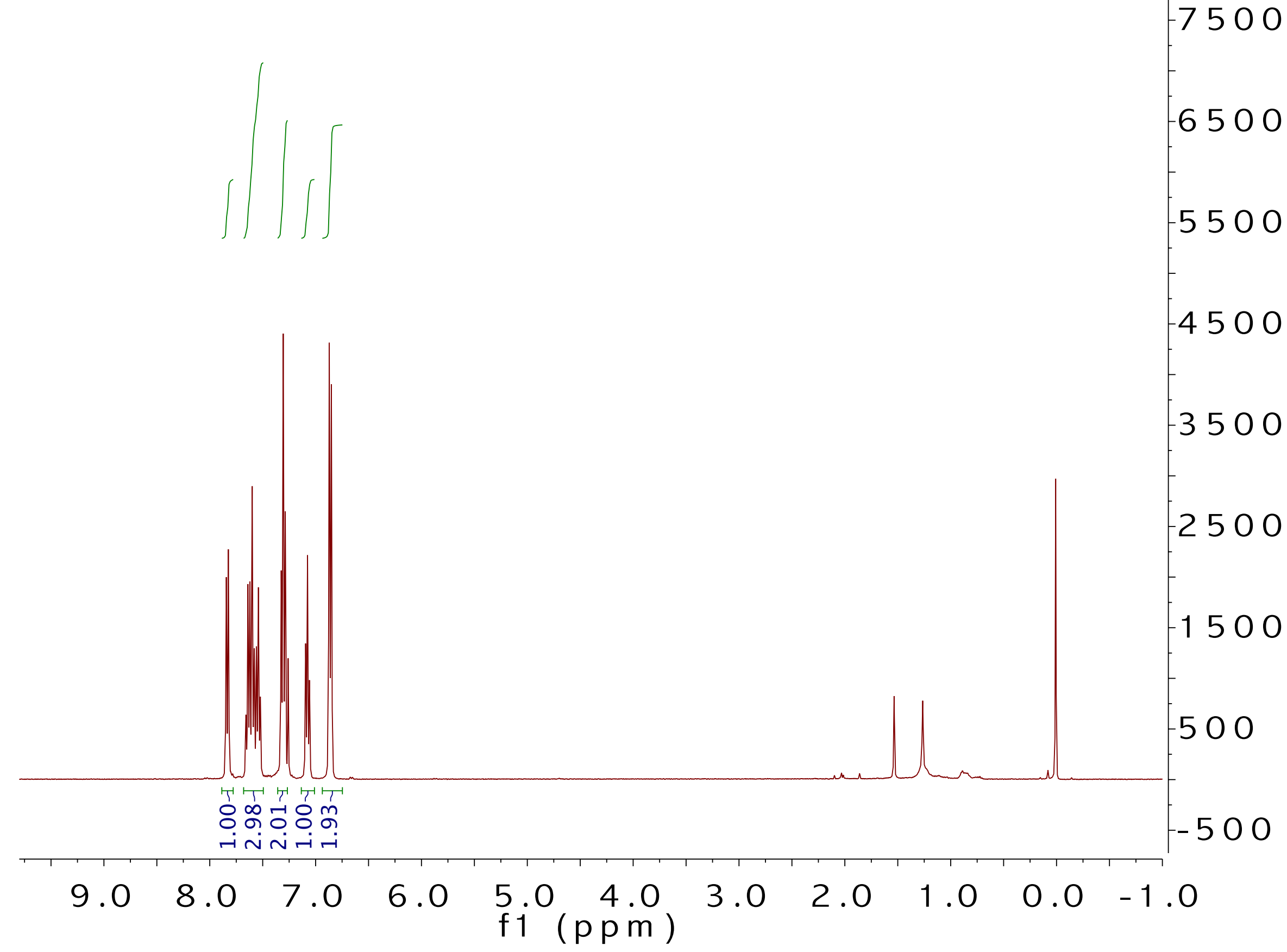




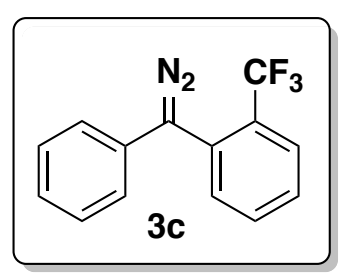

${ }^{13} \mathrm{C}, 100 \mathrm{MHz}, \mathrm{CDCl}_{3}$ 


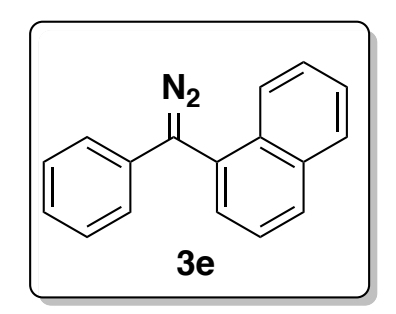

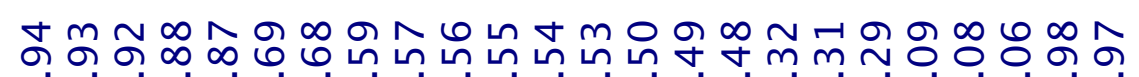

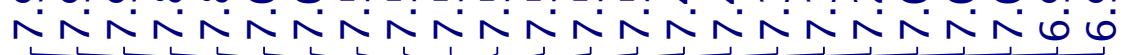

${ }^{1} \mathrm{H}, 600 \mathrm{MHz}, \mathrm{CDCl}_{3}$

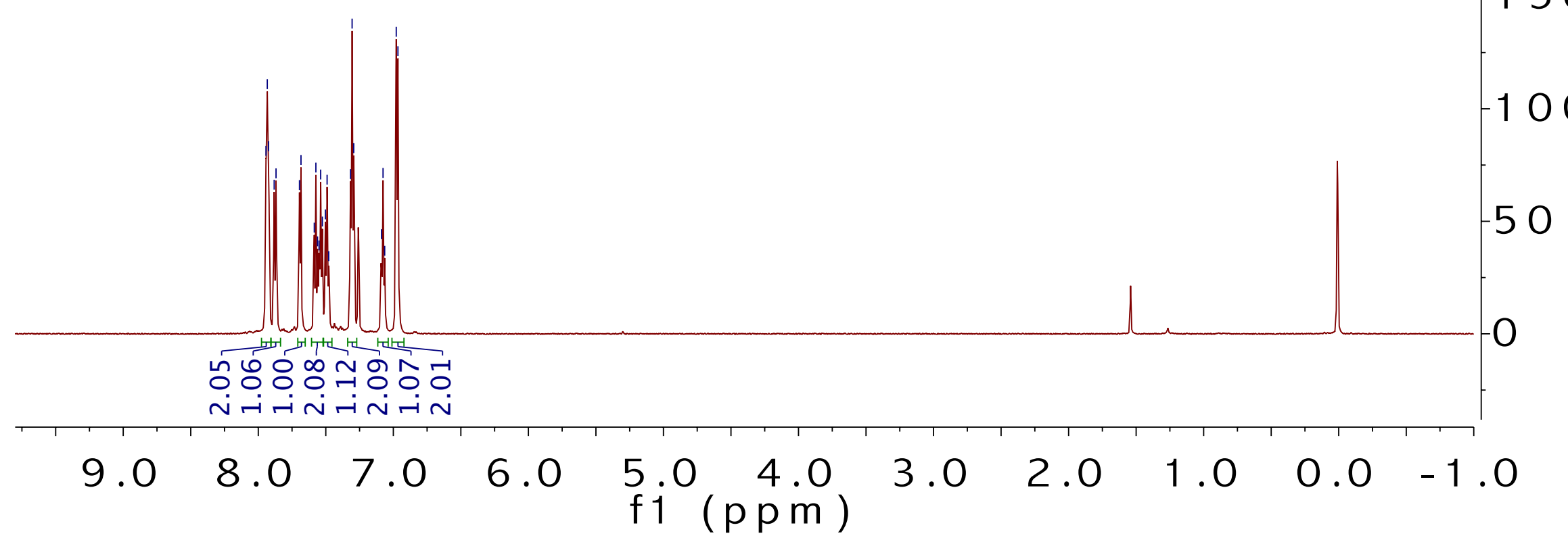



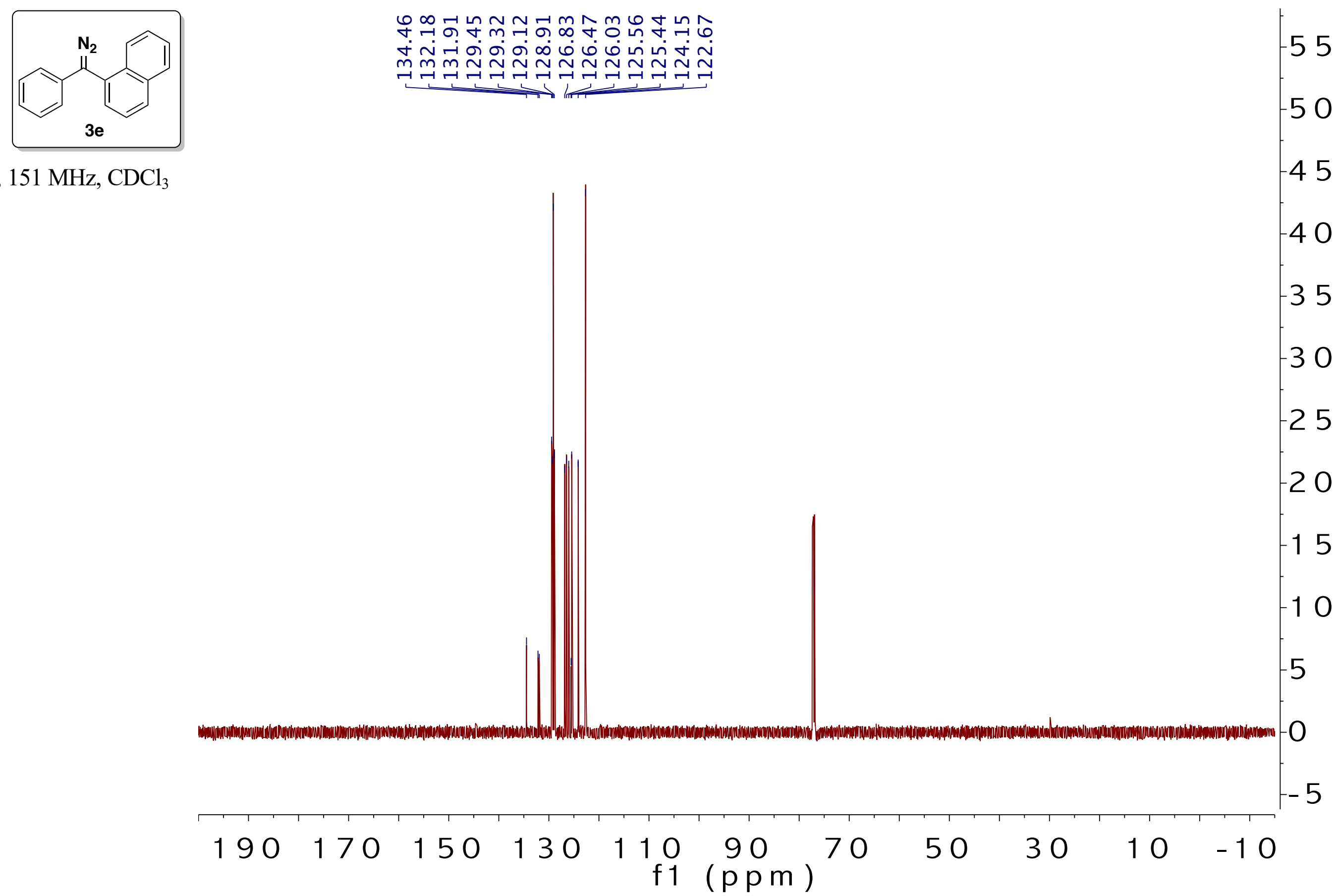

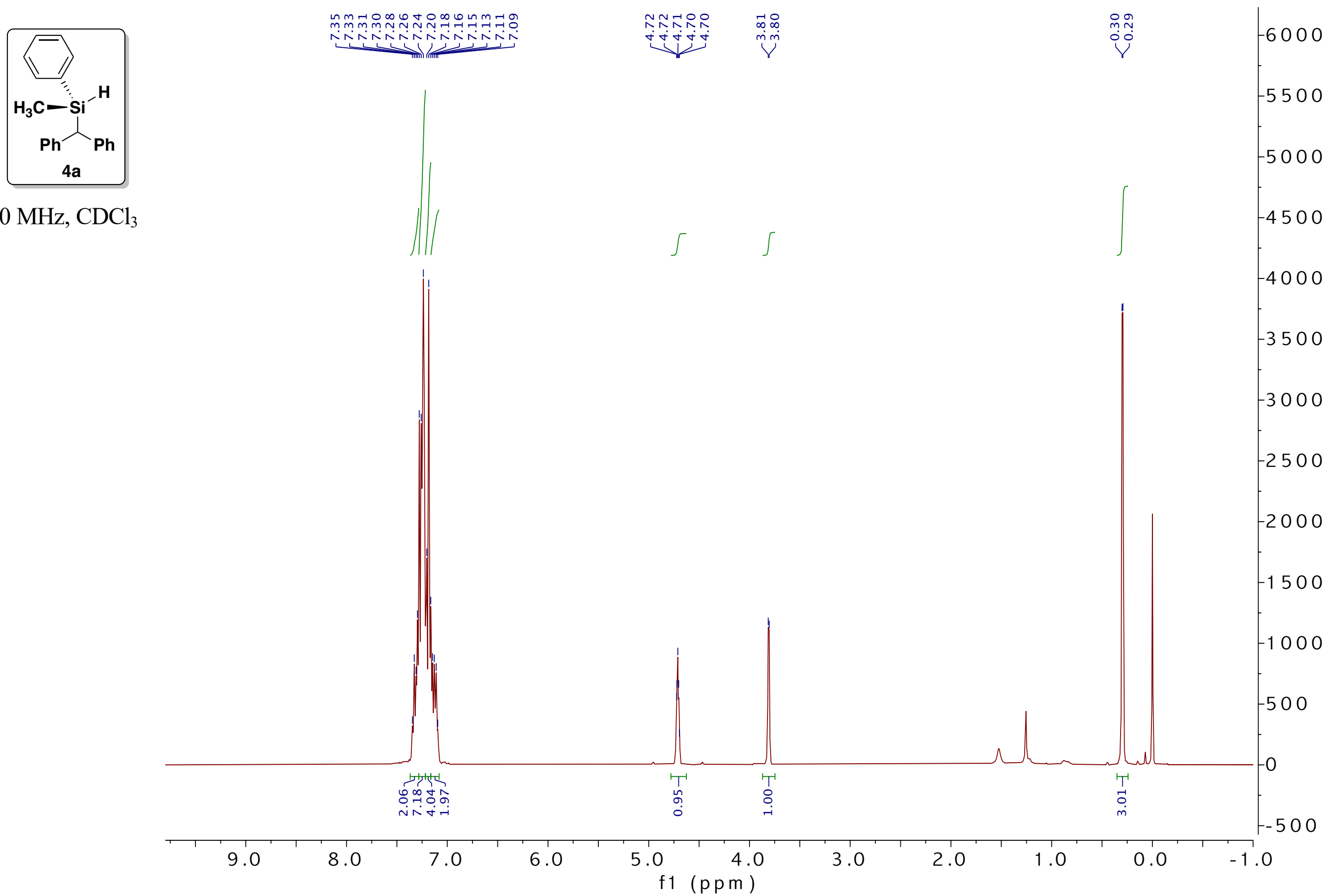

6000

5000

${ }^{1} \mathrm{H}, 400 \mathrm{MHz}, \mathrm{CDCl}_{3}$ f1 (ppm) 


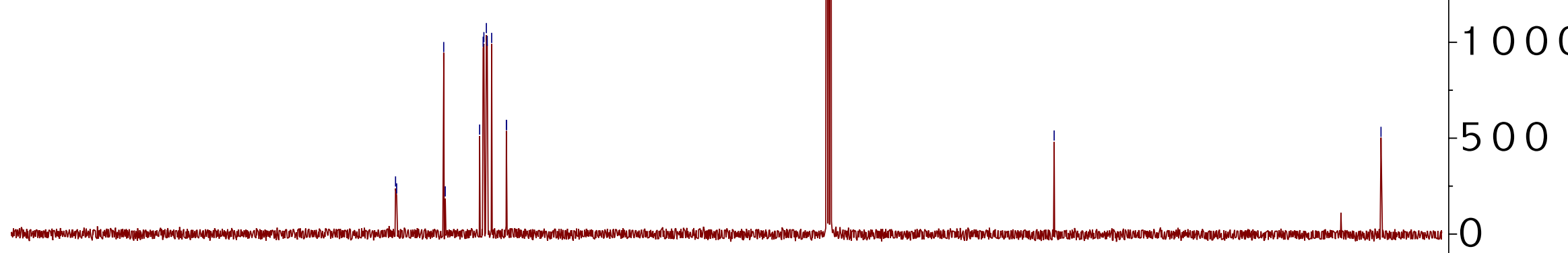




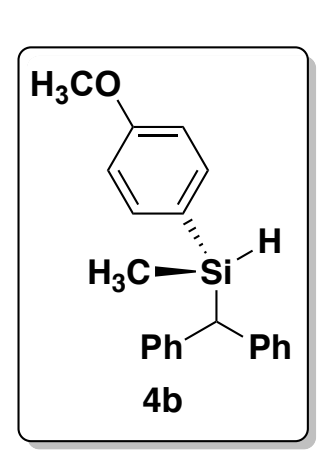

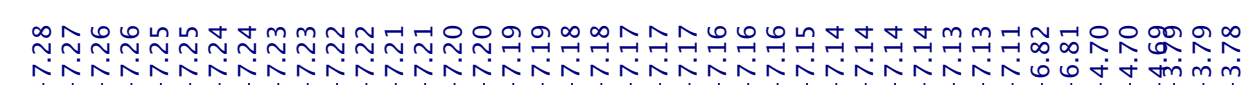

${ }^{1} \mathrm{H}, 600 \mathrm{MHz}, \mathrm{CDCl}_{3}$

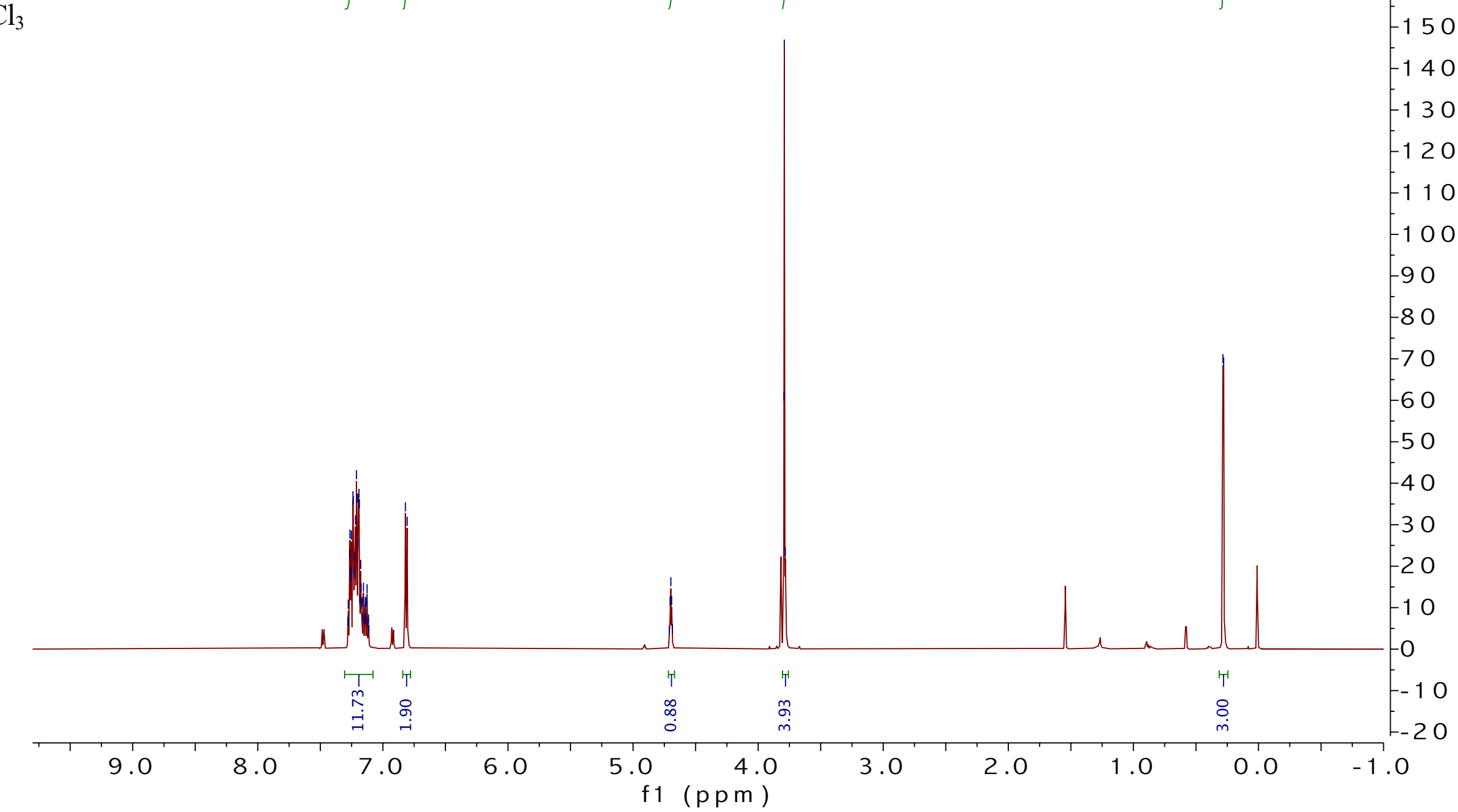




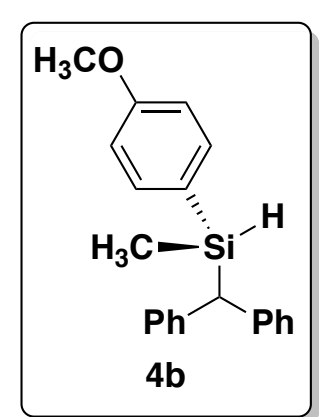

${ }^{13} \mathrm{C}, 151 \mathrm{MHz}, \mathrm{CDCl}_{3}$

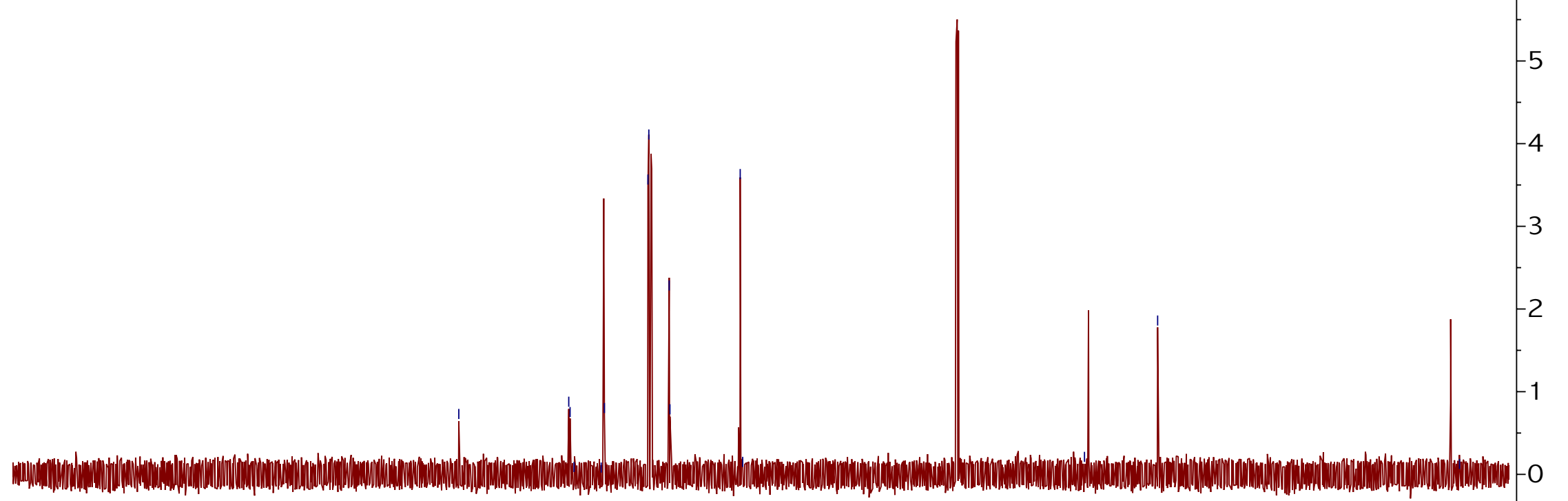




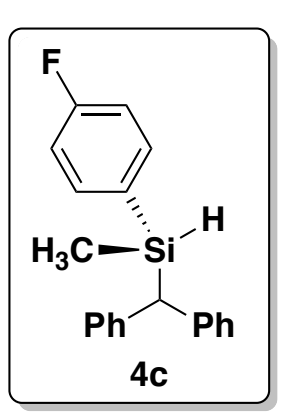

$m$ m

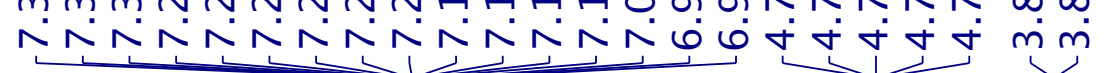

${ }^{1} \mathrm{H}, 400 \mathrm{MHz}, \mathrm{CDCl}_{3}$

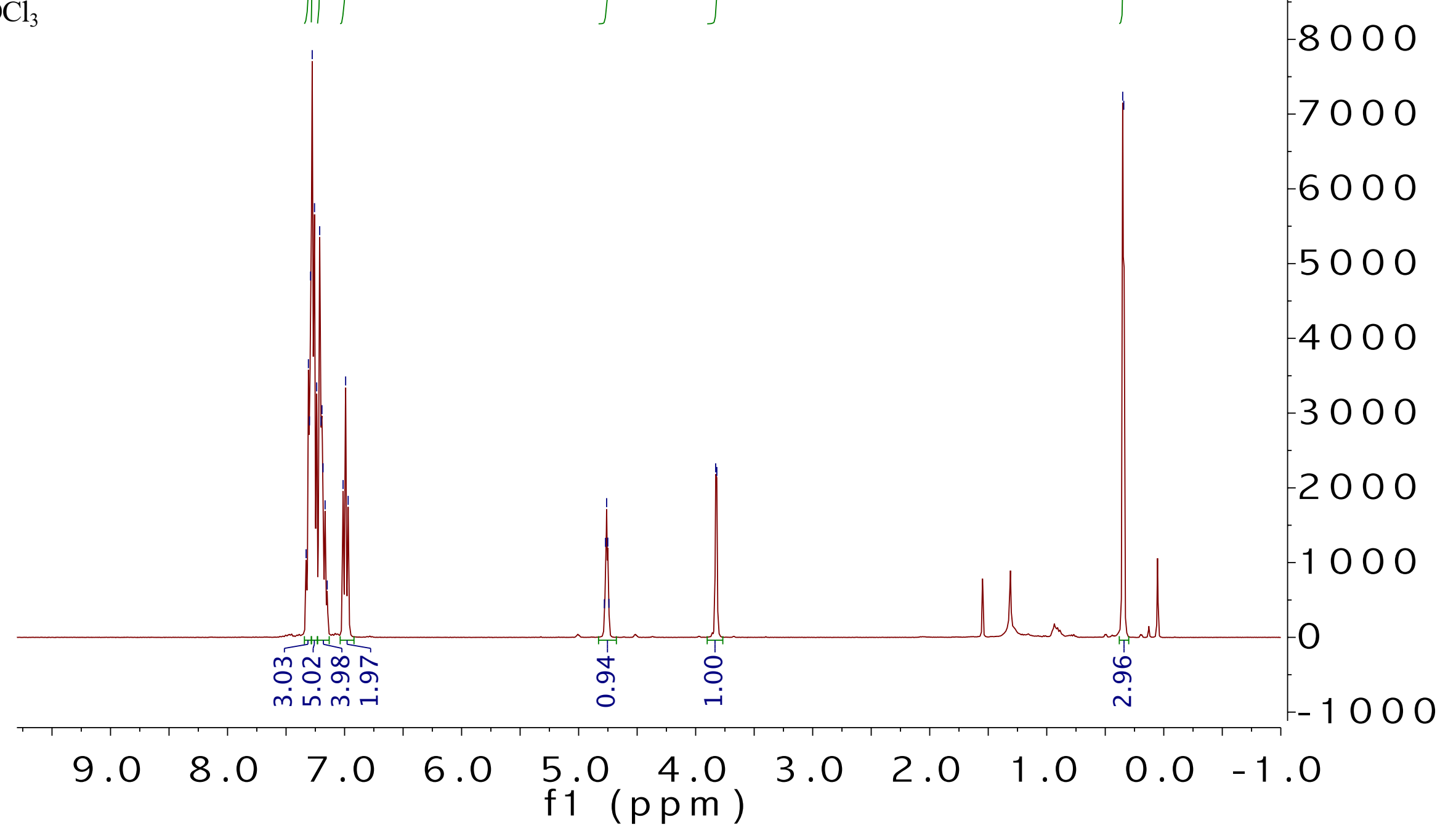




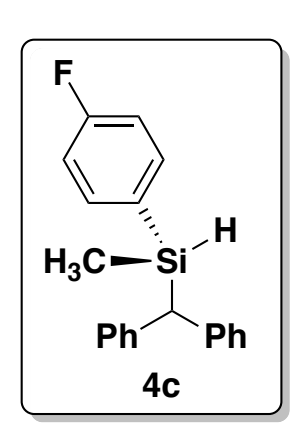

ํㅅㅅำ

ம்

${ }^{13} \mathrm{C}, 100 \mathrm{MHz}, \mathrm{CDCl}_{3}$

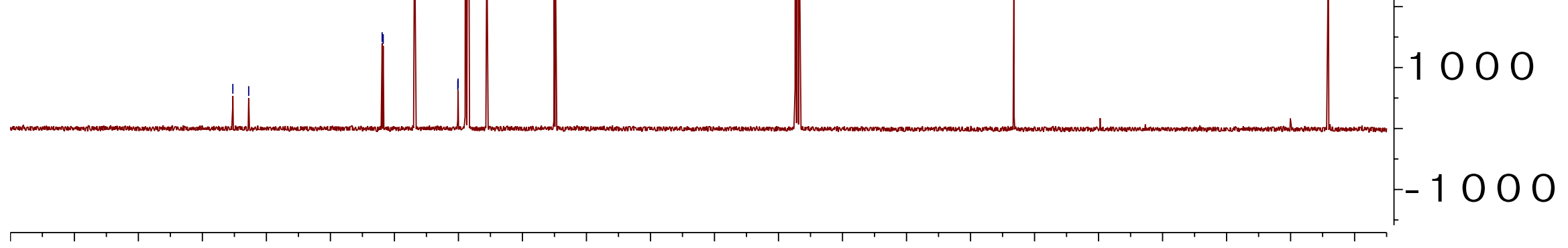



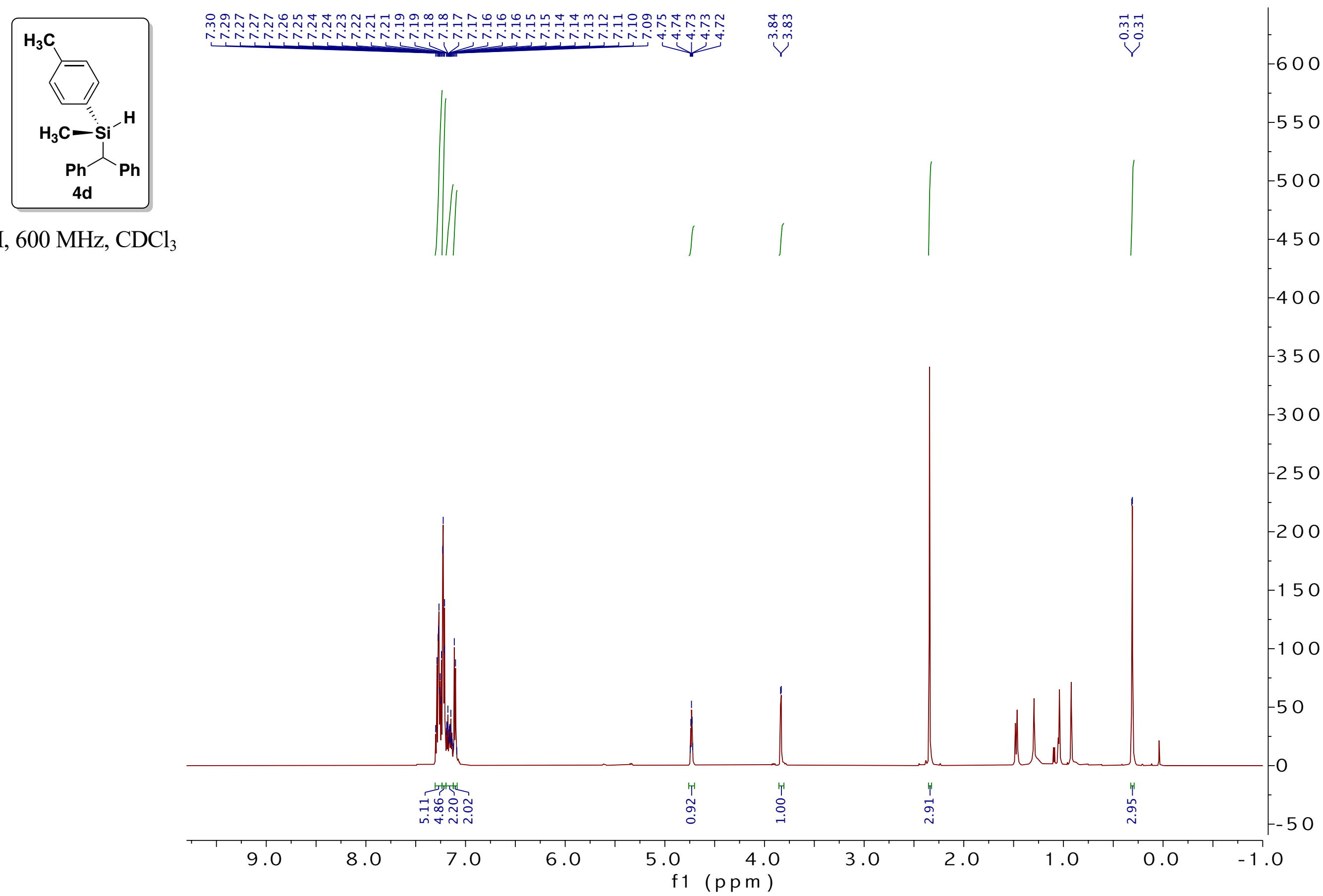


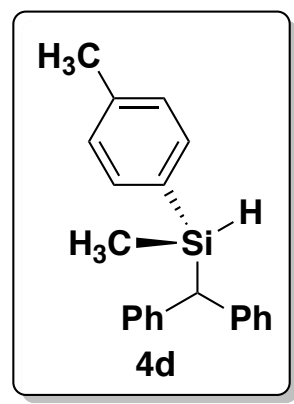

${ }^{13} \mathrm{C}, 151 \mathrm{MHz}, \mathrm{CDCl}_{3}$

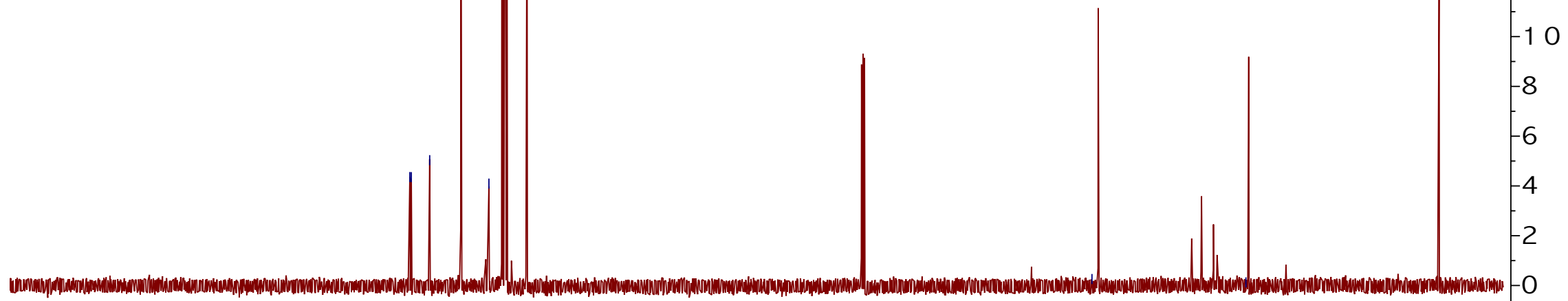




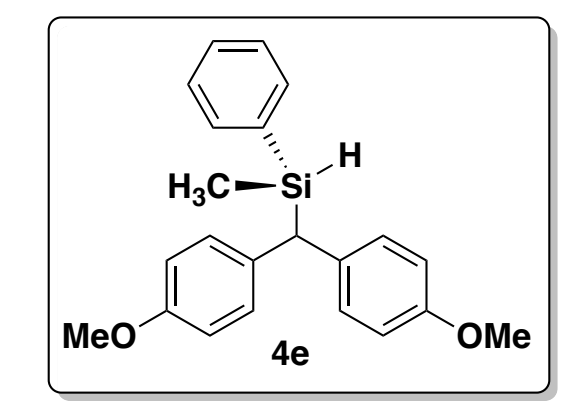

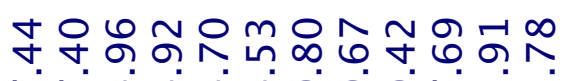

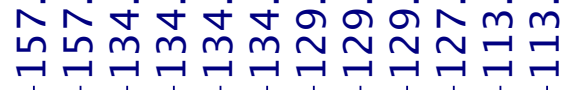

$\stackrel{\sim}{\sim}$

กิ่นกี่

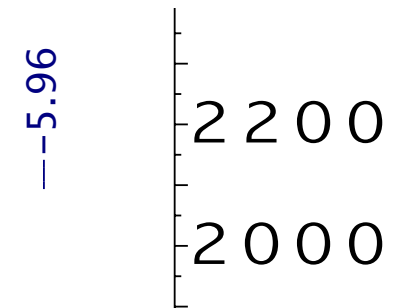

${ }^{13} \mathrm{C}, 100 \mathrm{MHz}, \mathrm{CDCl}_{3}$ 


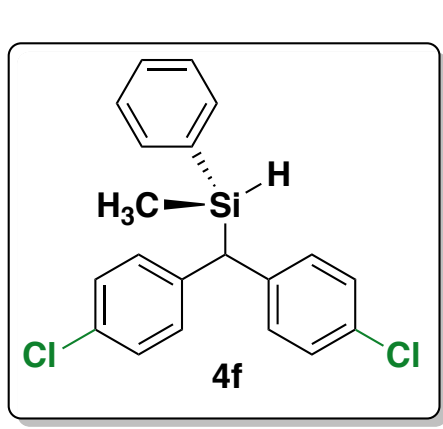

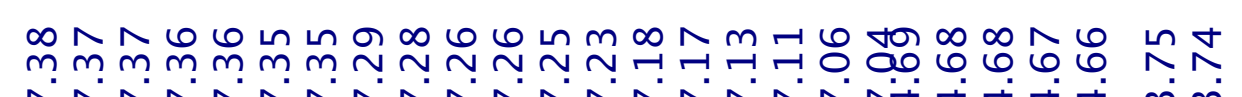
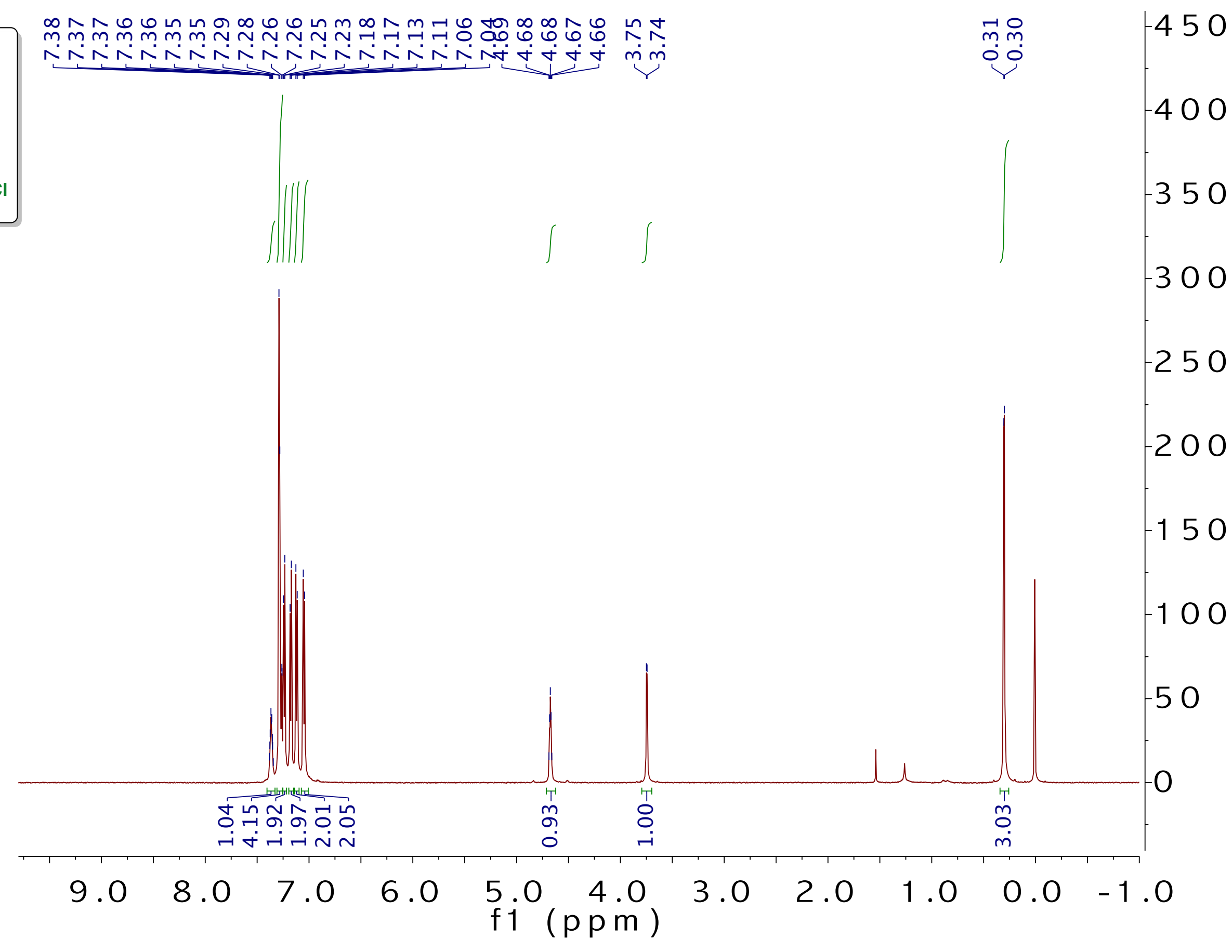

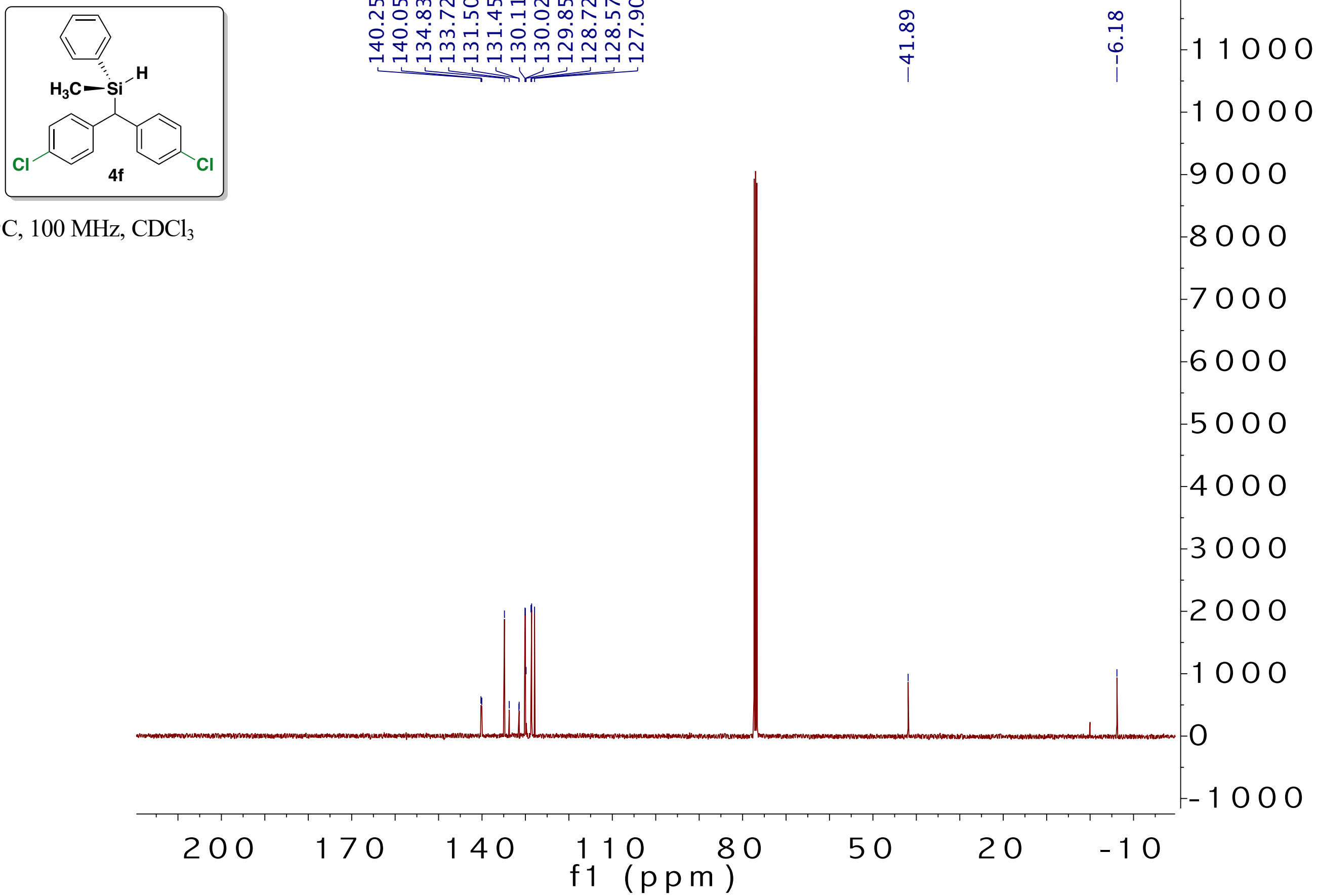


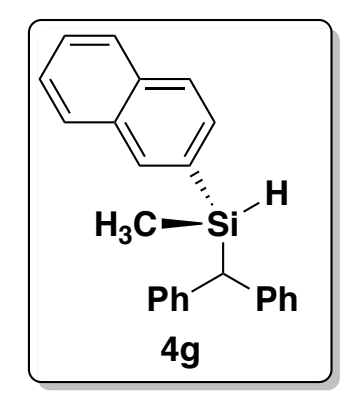

${ }^{13} \mathrm{C}, 151 \mathrm{MHz}, \mathrm{CDCl}_{3}$

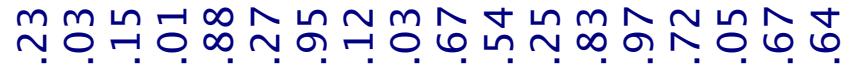

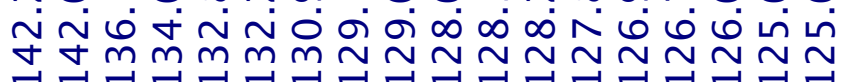

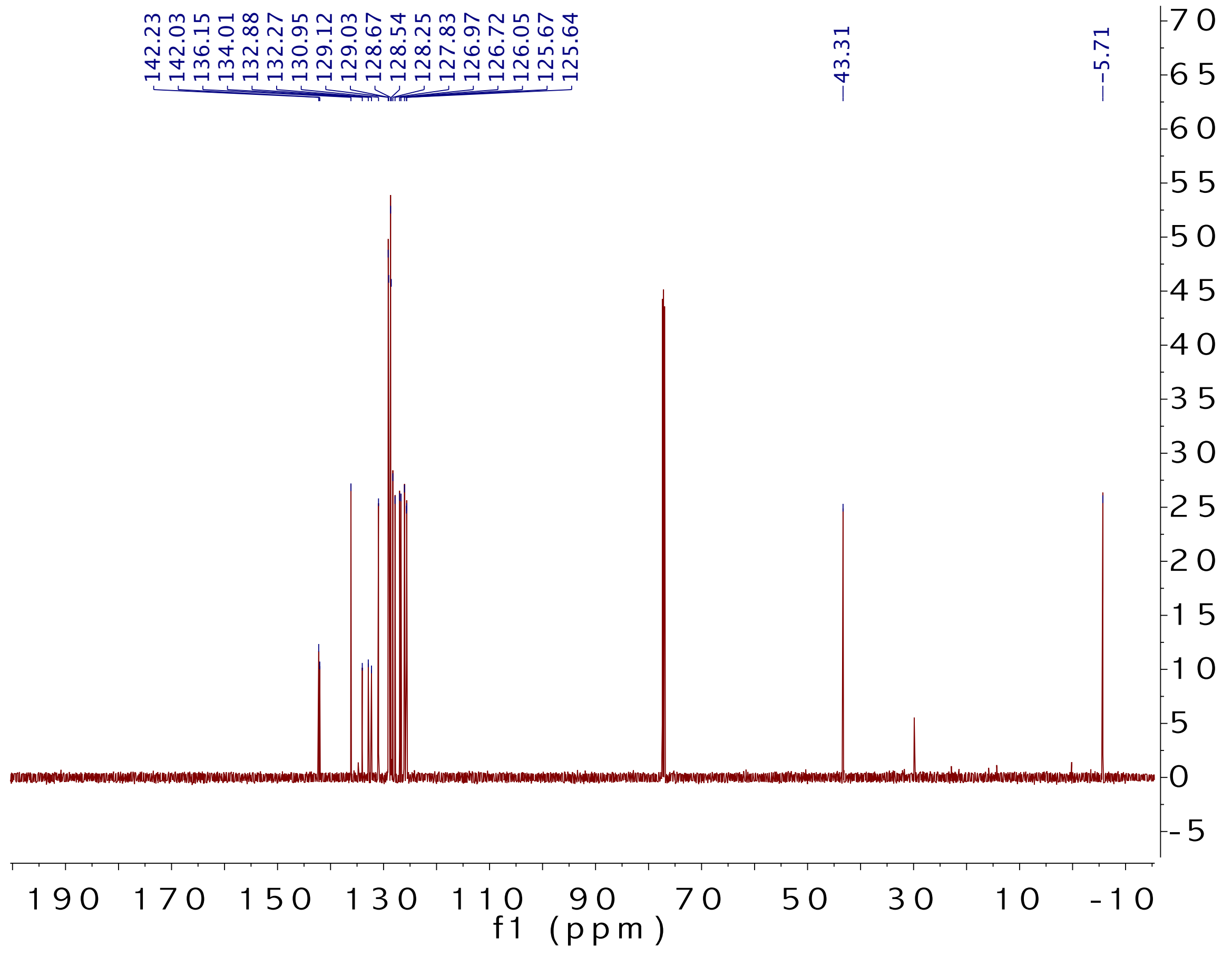

$-5$

S119 


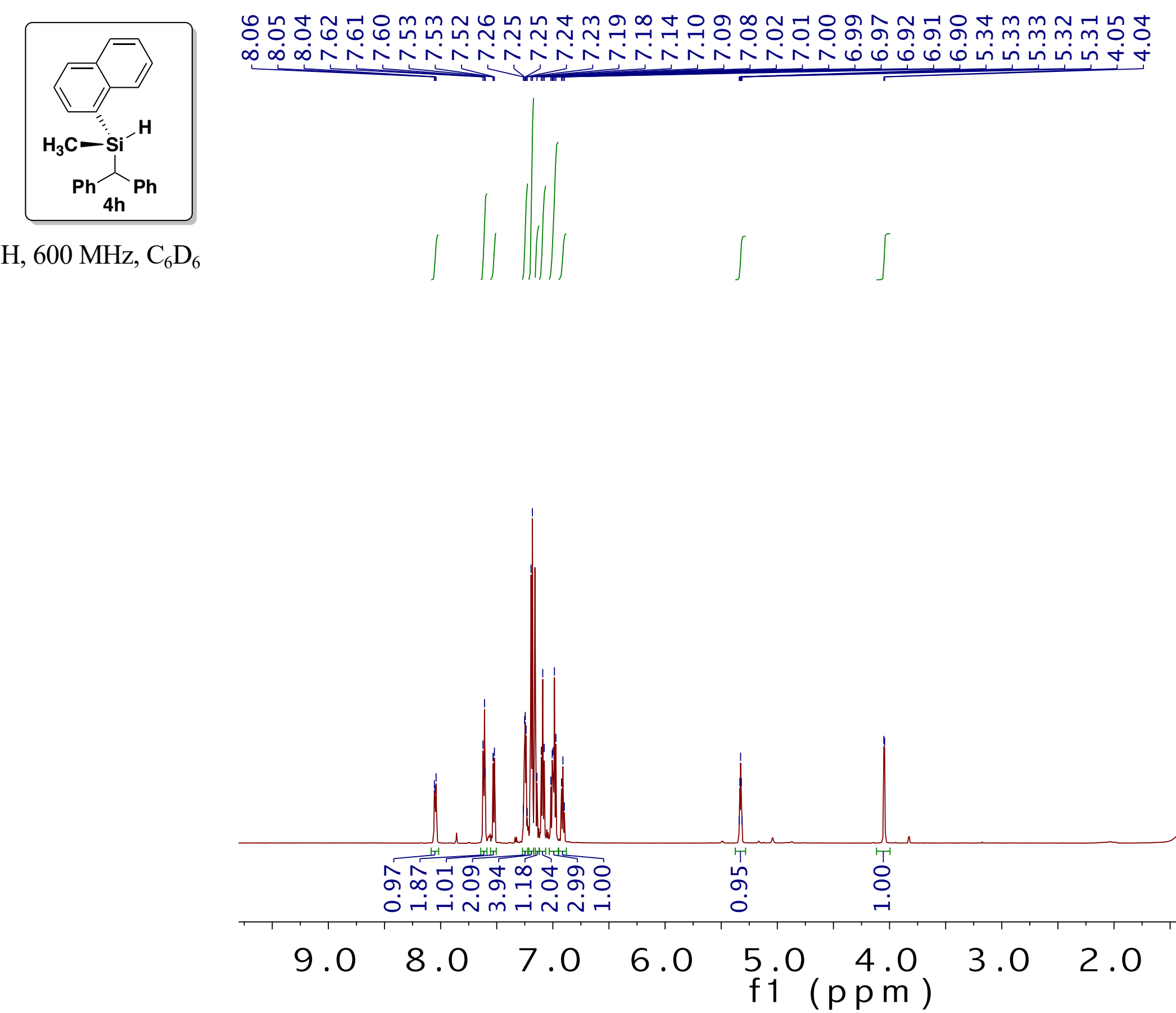

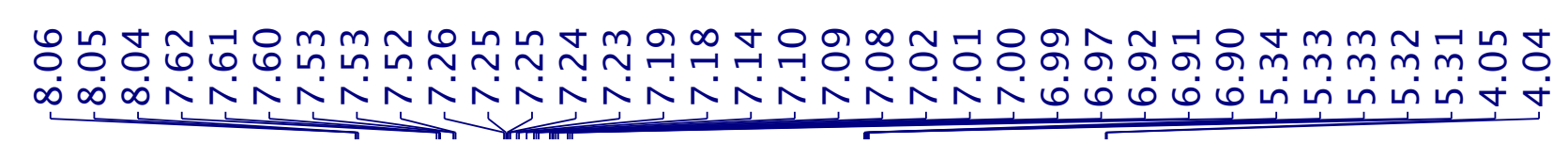




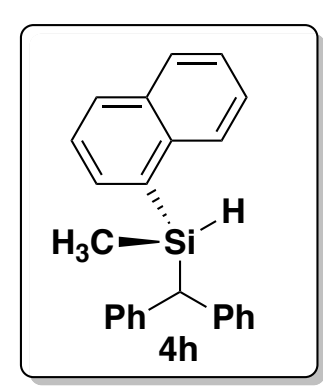

${ }^{13} \mathrm{C}, 100 \mathrm{MHz}, \mathrm{CDCl}_{3}$

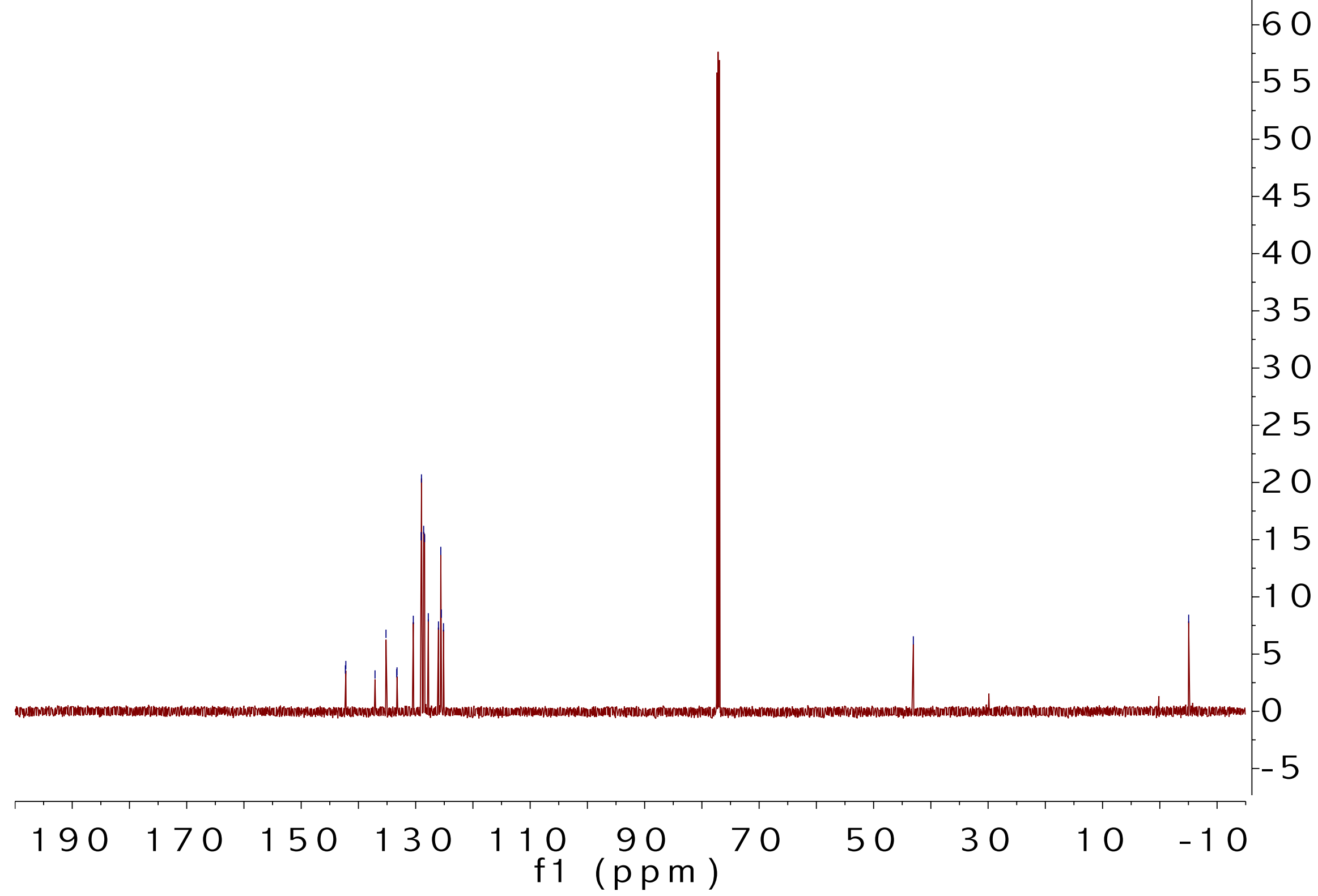




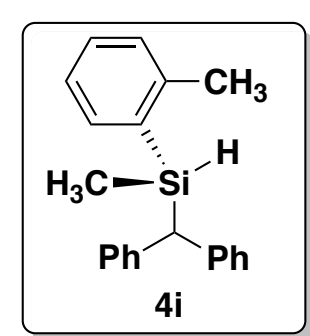

${ }^{1} \mathrm{H}, 600 \mathrm{MHz}, \mathrm{CDCl}_{3}$

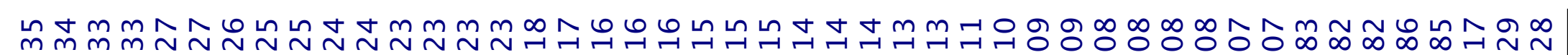

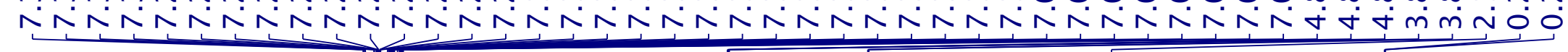

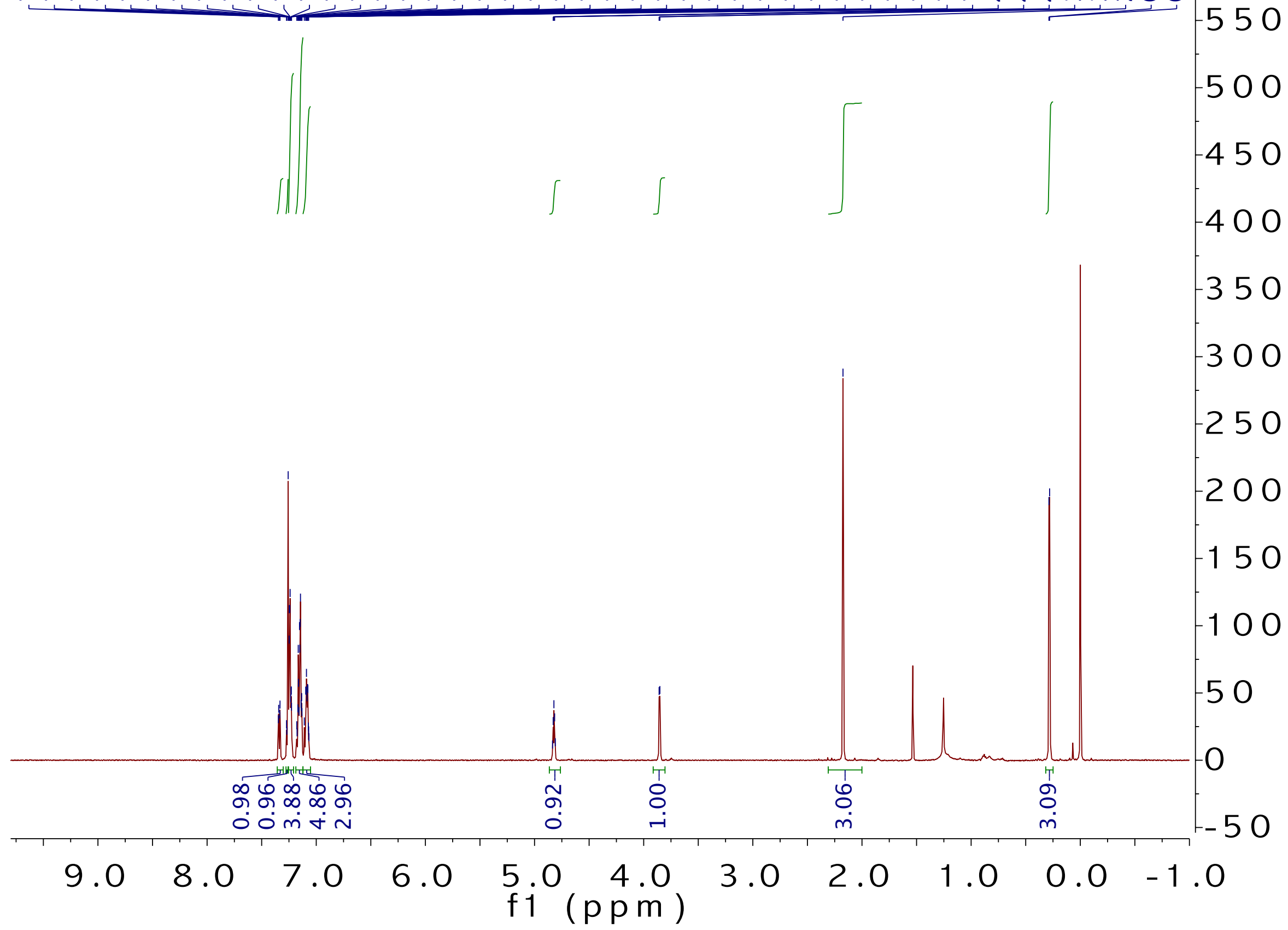




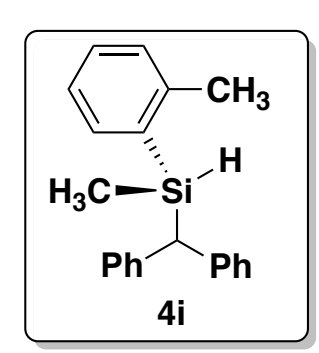



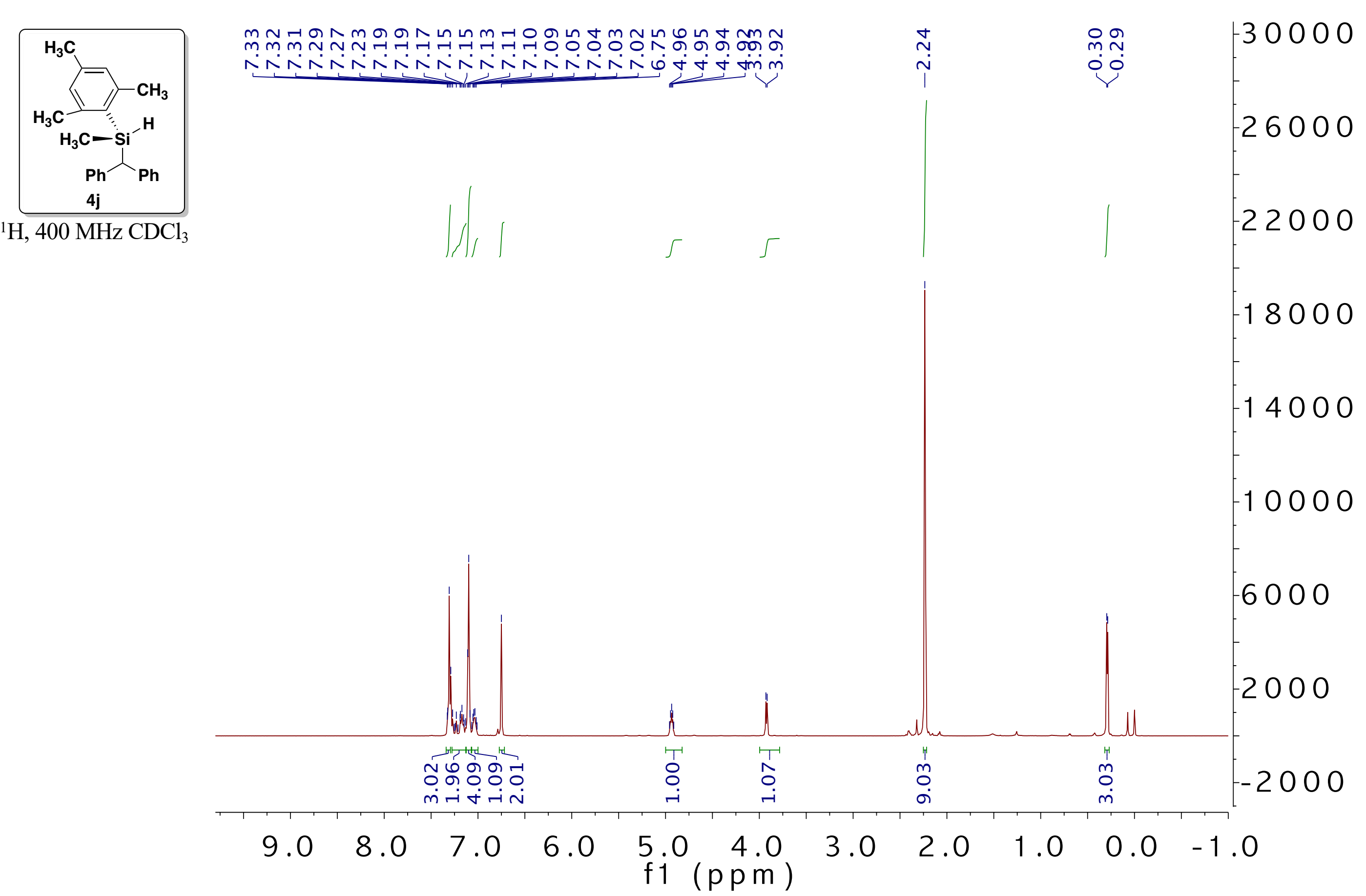


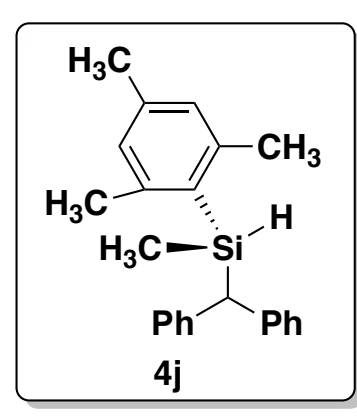



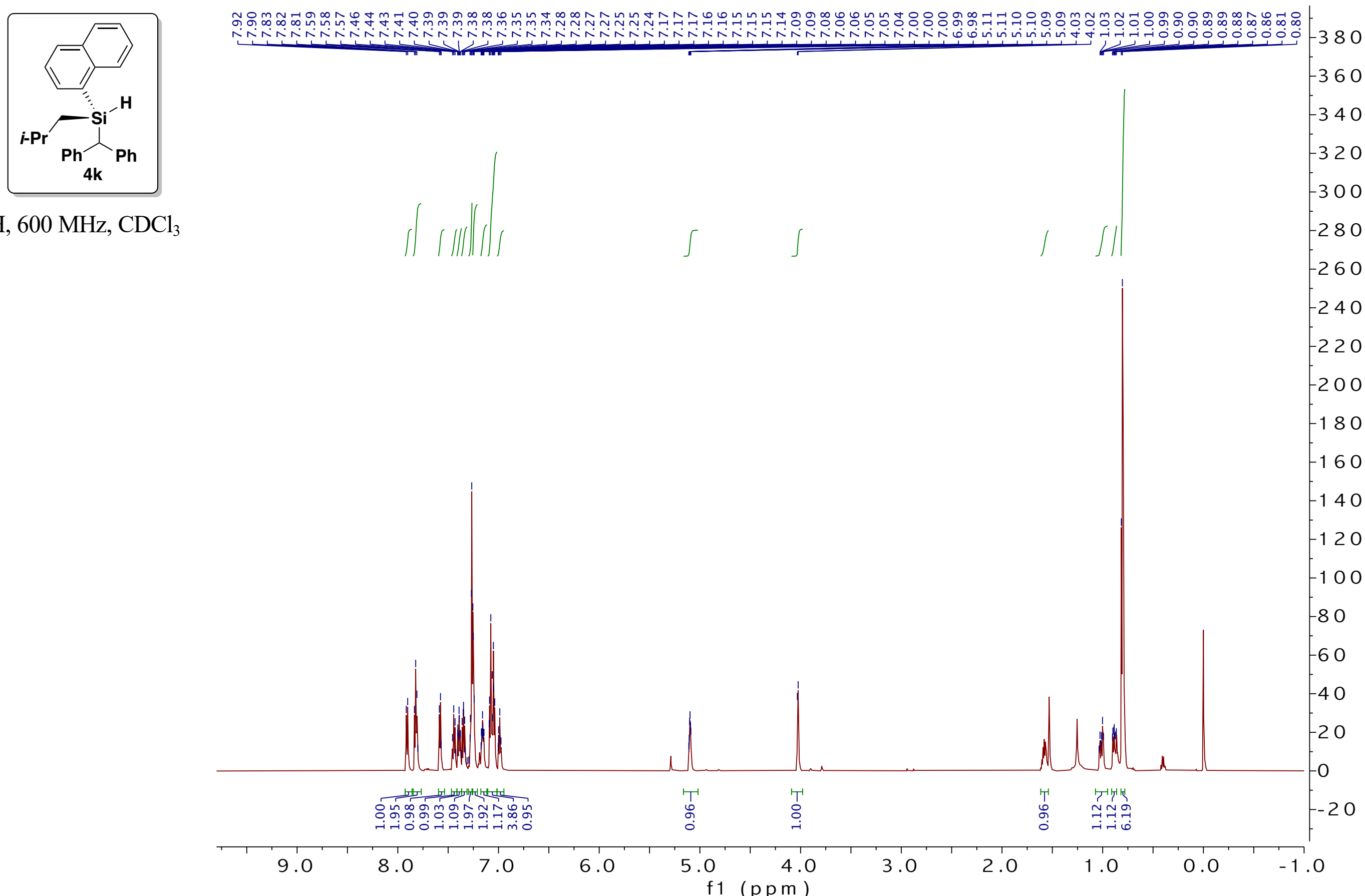


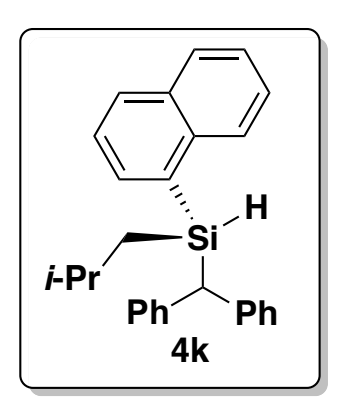

${ }^{13} \mathrm{C}, 151 \mathrm{MHz}, \mathrm{CDCl}_{3}$ 


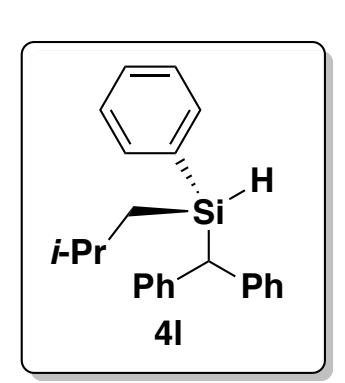

${ }^{1} \mathrm{H}, 400 \mathrm{MHz}, \mathrm{CDCl}_{3}$

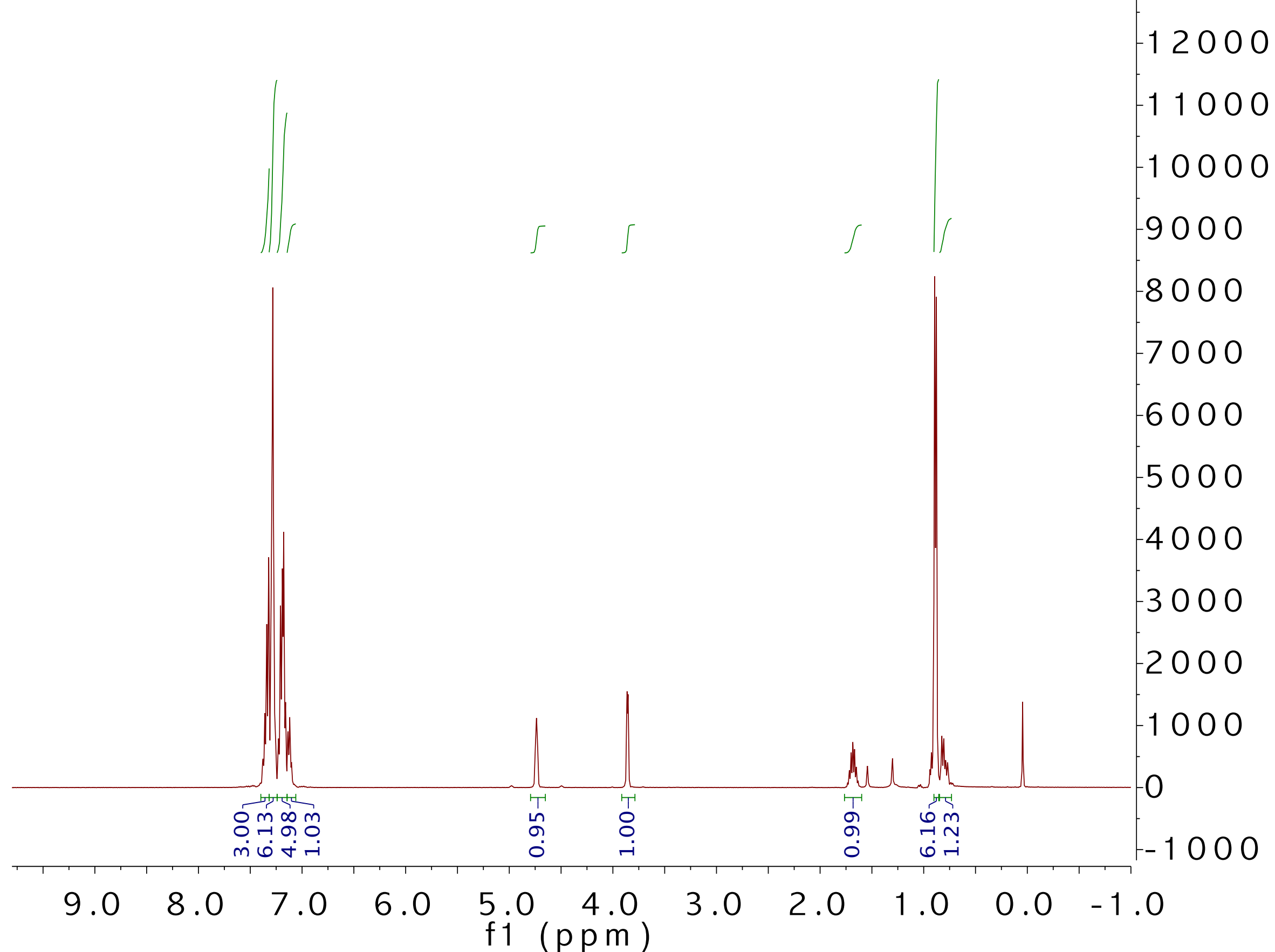




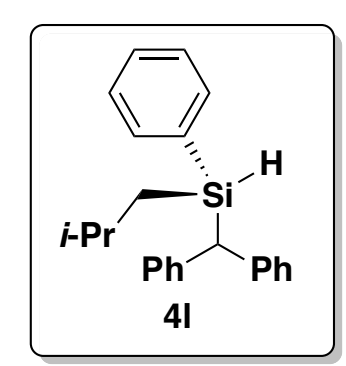

${ }^{13} \mathrm{C}, 100 \mathrm{MHz}, \mathrm{CDCl}_{3}$

4500

4000

3500

3000

2500

$-2000$

1500

1000

500

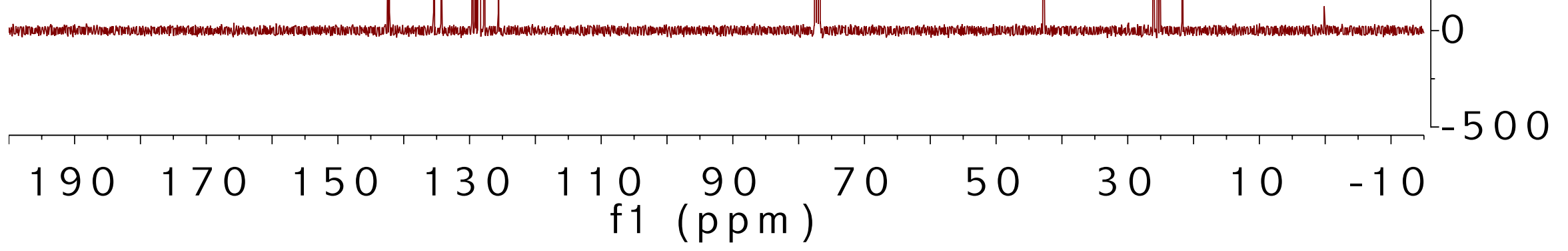




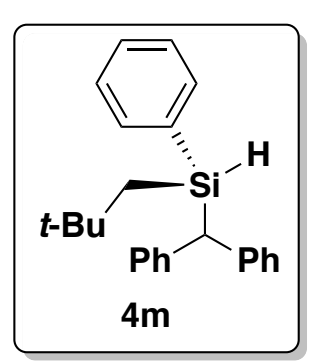

${ }^{1} \mathrm{H}, 400 \mathrm{MHz}, \mathrm{CDCl}_{3}$

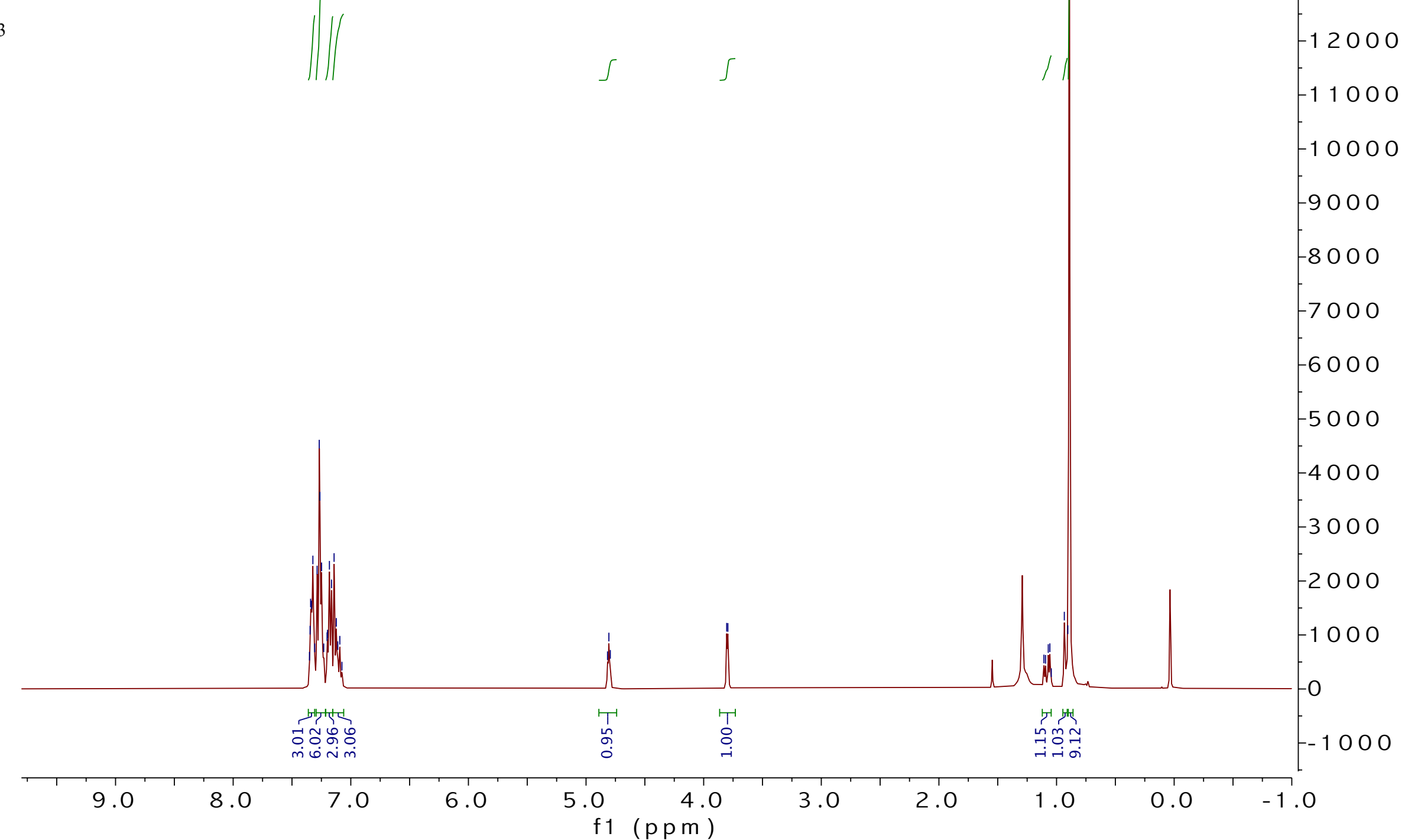




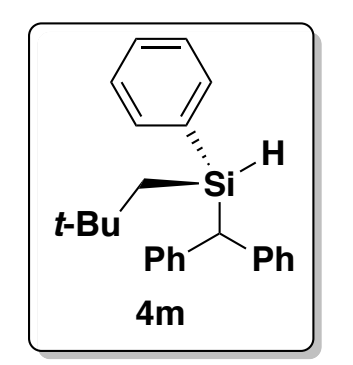

${ }^{13} \mathrm{C}, 100 \mathrm{MHz}, \mathrm{CDCl}_{3}$

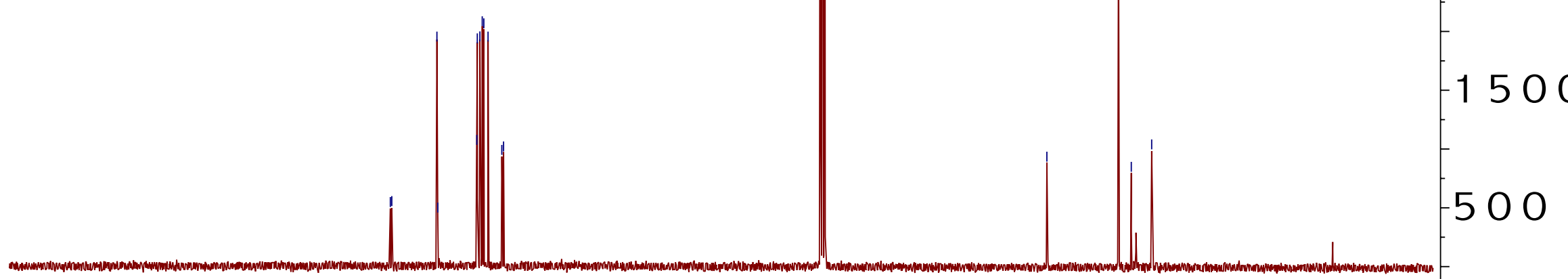




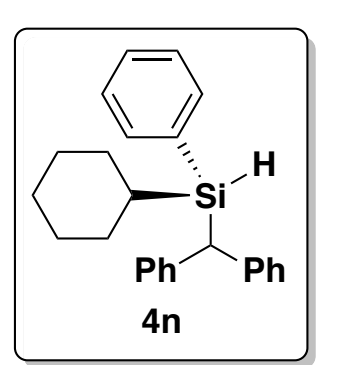

${ }^{1} \mathrm{H}, 600 \mathrm{MHz}, \mathrm{CDCl}_{3}$

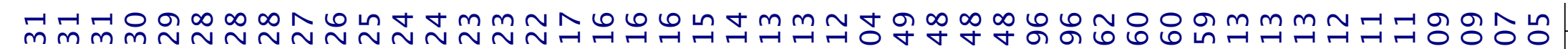

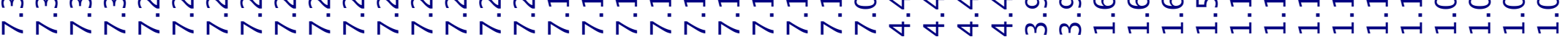

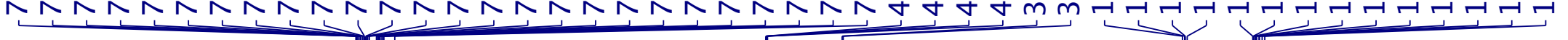
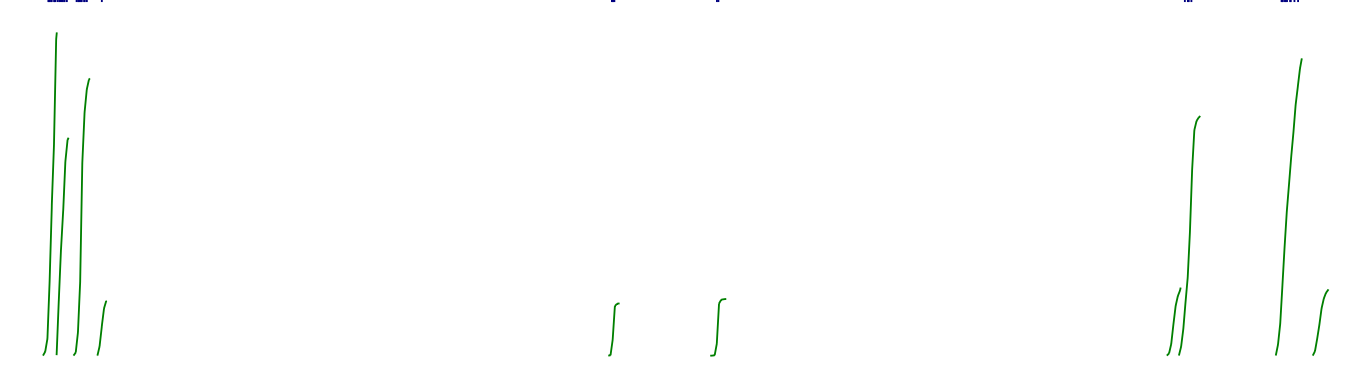

$-300$

260

220

180

140

100

60

$-20$ $-20$
9.0
8.0
$7.0 \quad 6.0$
${ }_{\mathrm{fi}}^{5}(\mathrm{ppm})$
3.0
2.0
$\begin{array}{llll}1.0 & 0.0 & -1.0\end{array}$ 


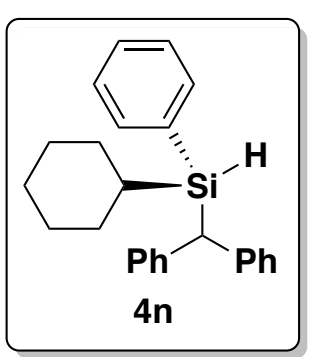



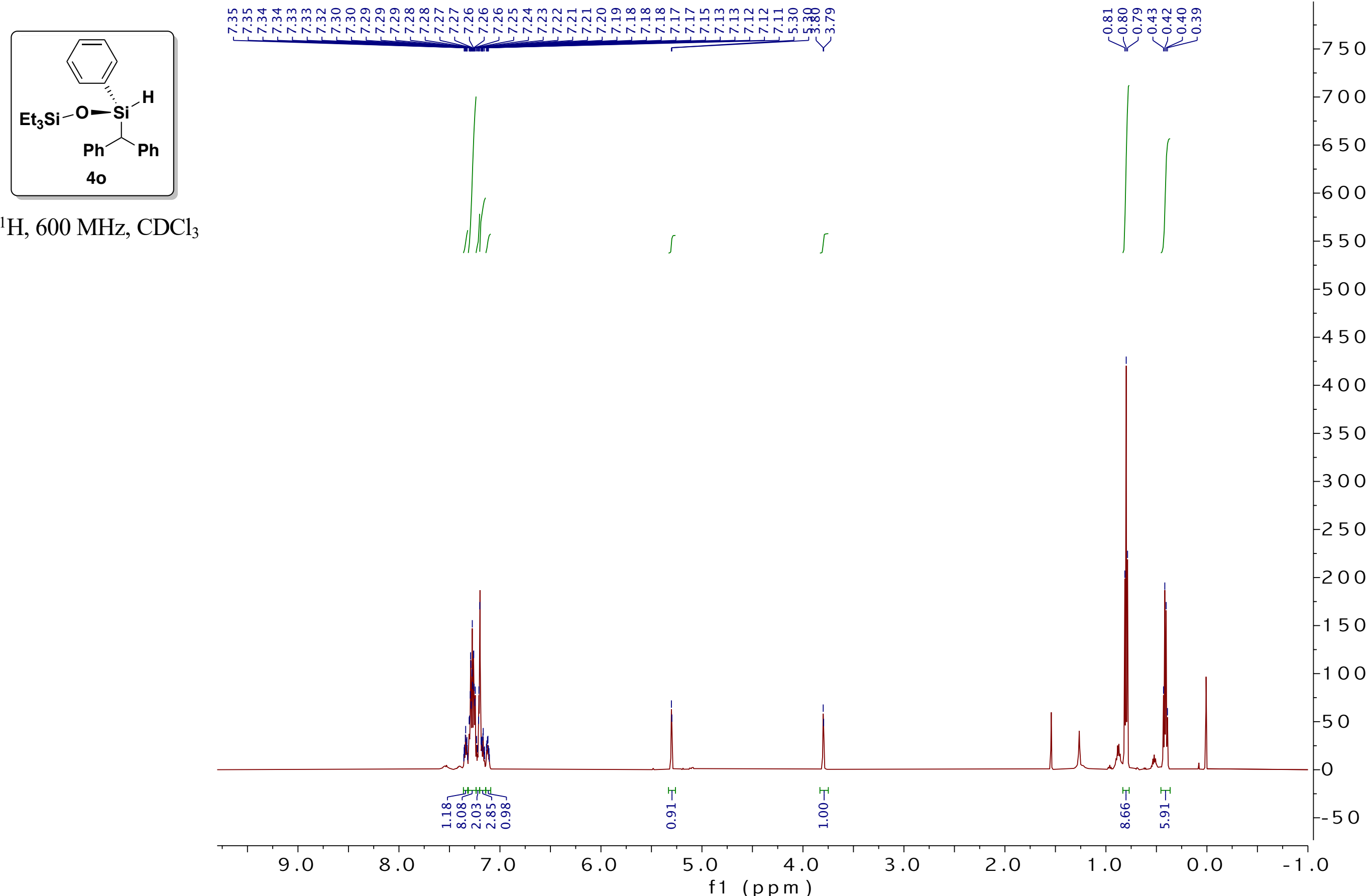


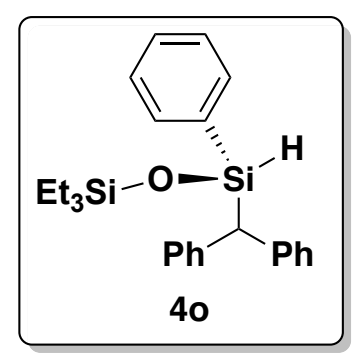

${ }^{13} \mathrm{C}, 151 \mathrm{MHz}, \mathrm{CDCl}_{3}$

$-9000$

$-8000$

$-7000$

$-6000$

5000

$-4000$

$-3000$

$-2000$

$-1000$ $-0$

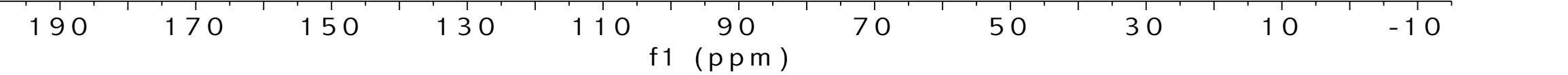




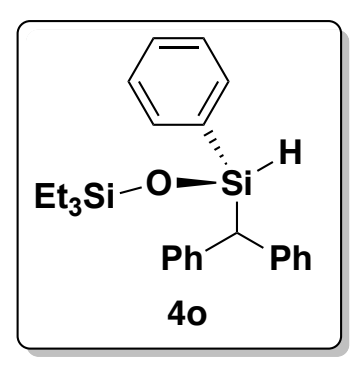

${ }^{29} \mathrm{Si}, 76 \mathrm{MHz}, \mathrm{CDCl}_{3}$

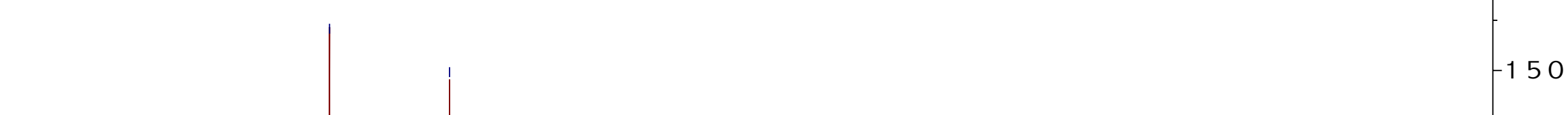

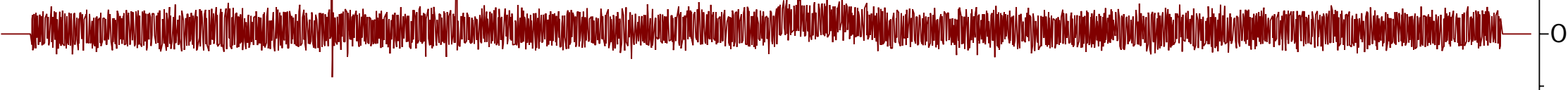




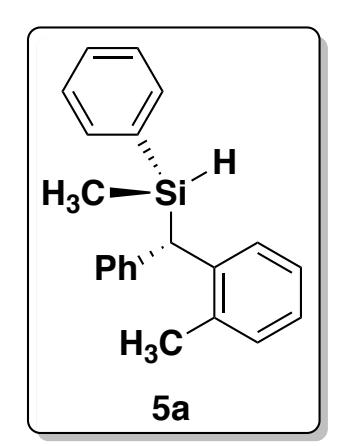

${ }^{13} \mathrm{C}, 150 \mathrm{MHz}, \mathrm{CDCl}_{3}$

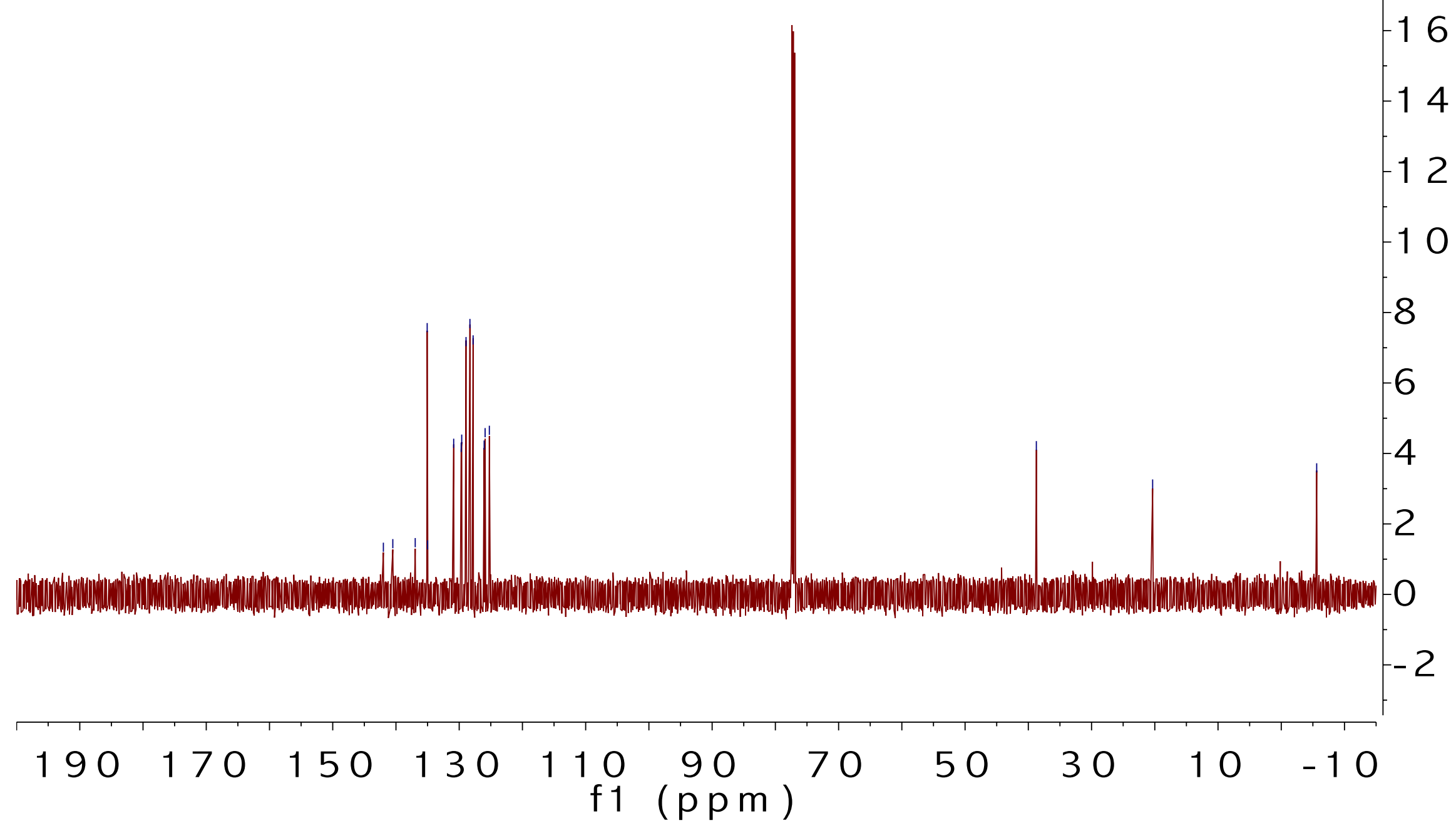




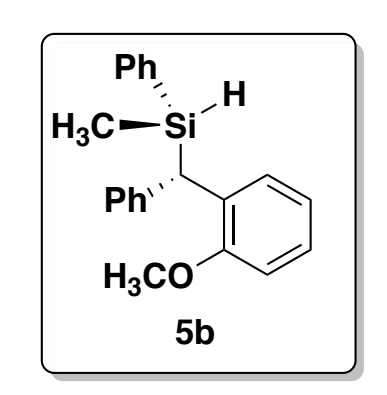

${ }^{1} \mathrm{H}, 600 \mathrm{MHz}, \mathrm{CDCl}_{3}$

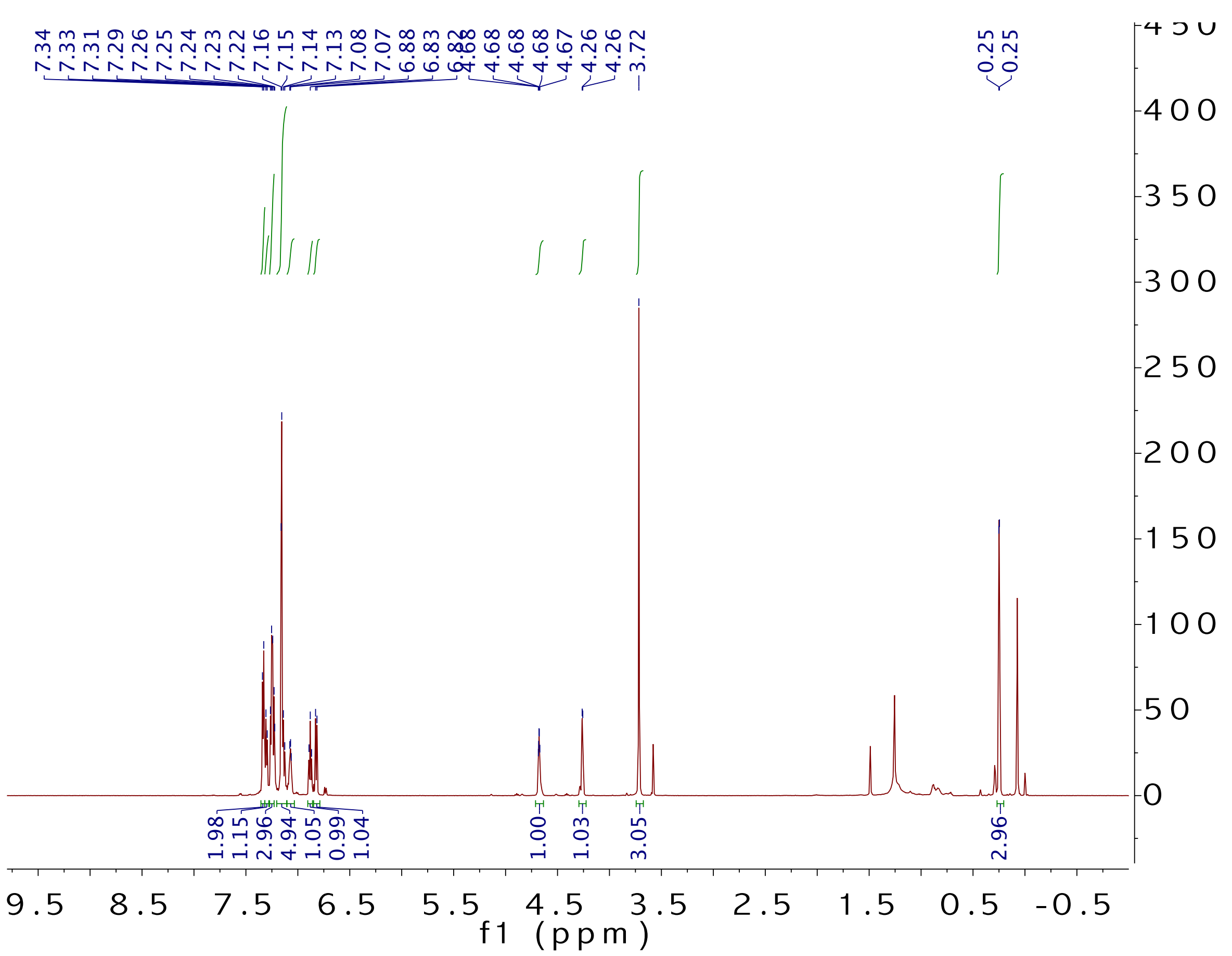




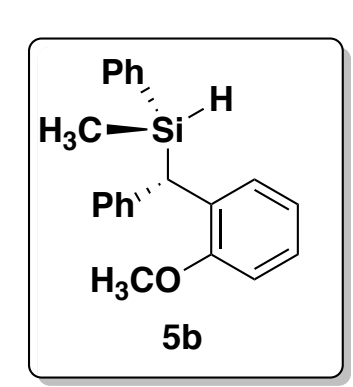

${ }^{13} \mathrm{C}, 151 \mathrm{MHz}, \mathrm{CDCl}_{3}$

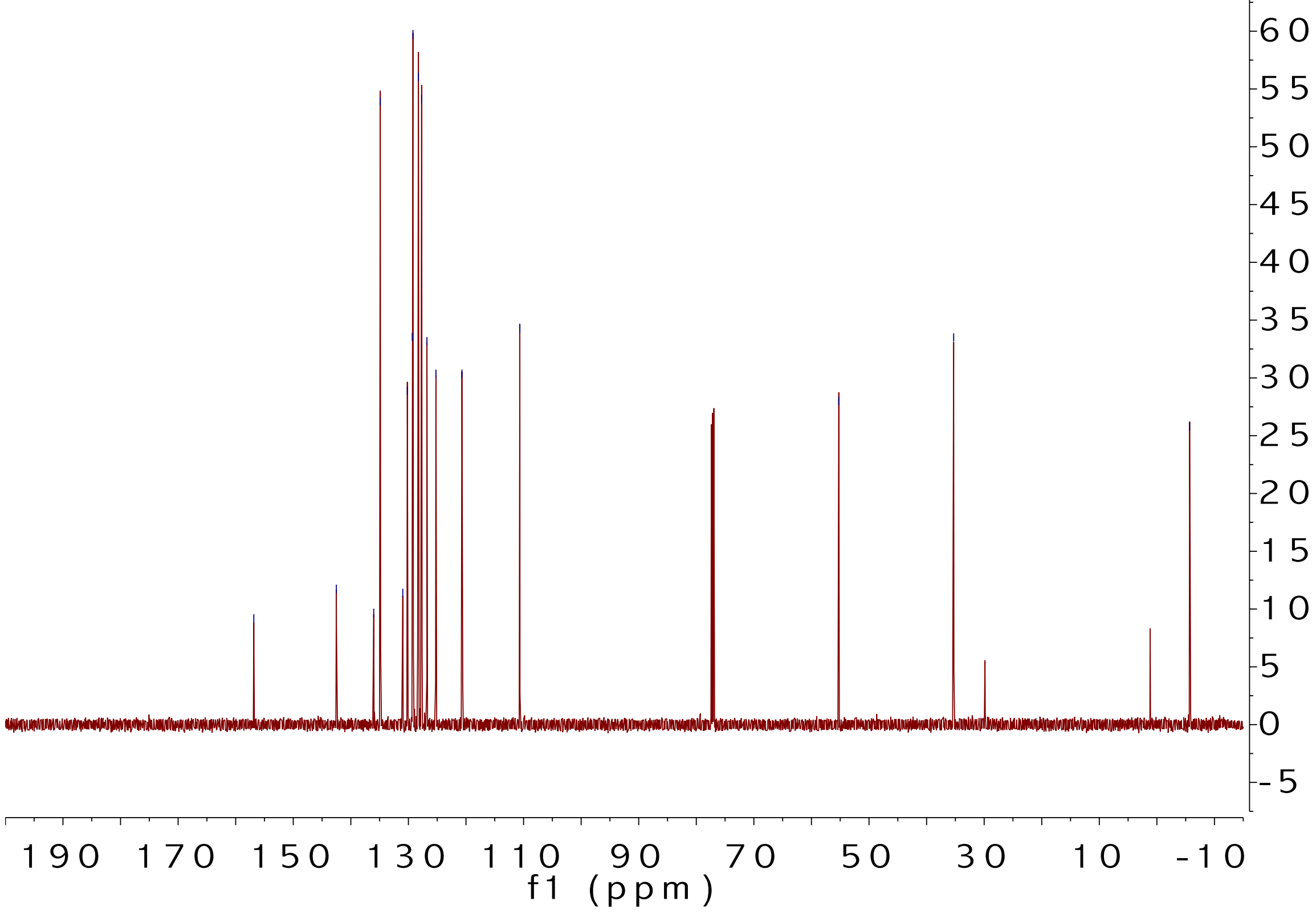




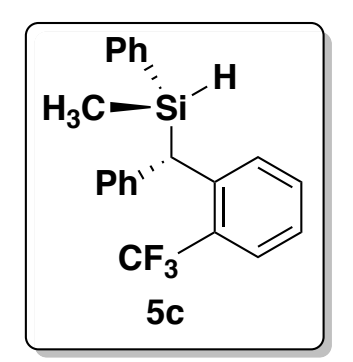

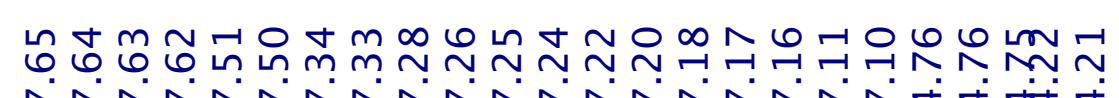

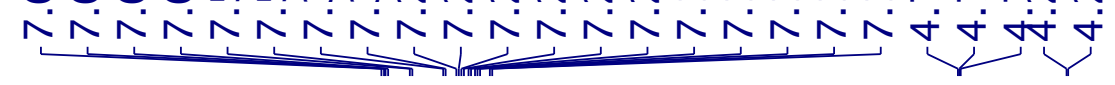

${ }^{1} \mathrm{H}, 600 \mathrm{MHz}, \mathrm{CDCl}_{3}$

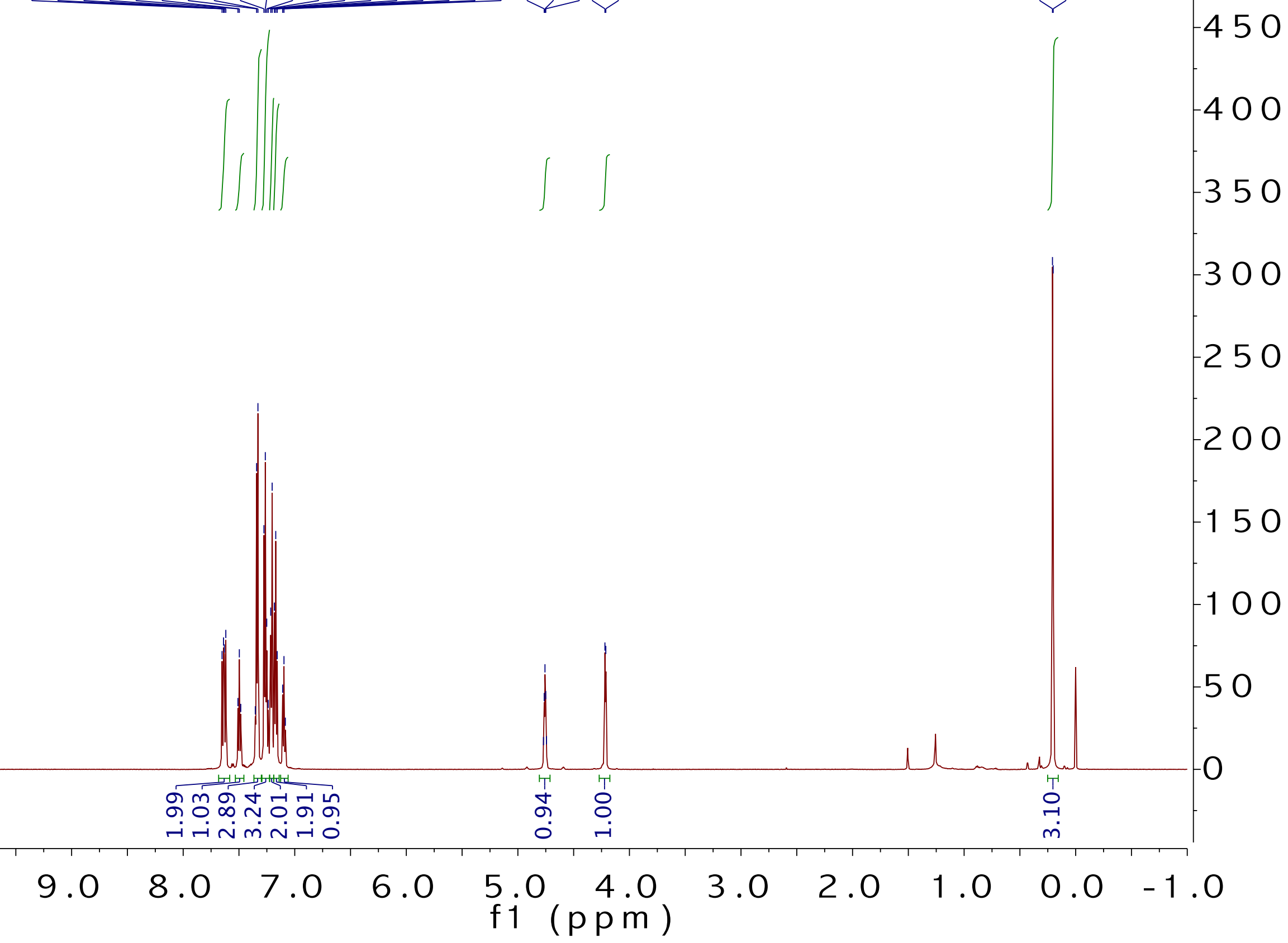


<smiles>CCc1ccccc1[C@H](c1ccccc1)[Si](C)(C)Cc1ccccc1</smiles>

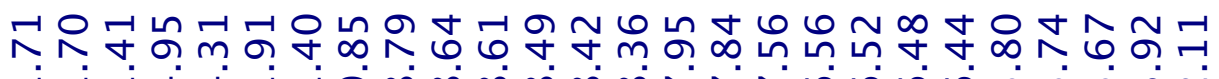

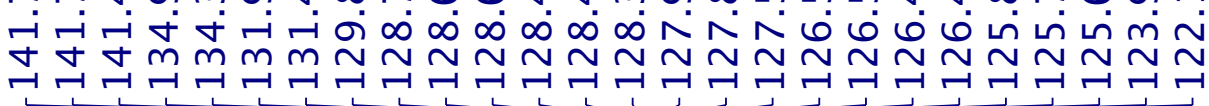

$\stackrel{\circ}{\infty}$

\begin{tabular}{l|lll}
$\stackrel{\infty}{\infty}$ & -1 & 1 & 0 \\
$\stackrel{\sim}{i}$ & -1 & 0 & 0
\end{tabular}

${ }^{13} \mathrm{C}, 151 \mathrm{MHz}, \mathrm{CDCl}_{3}$

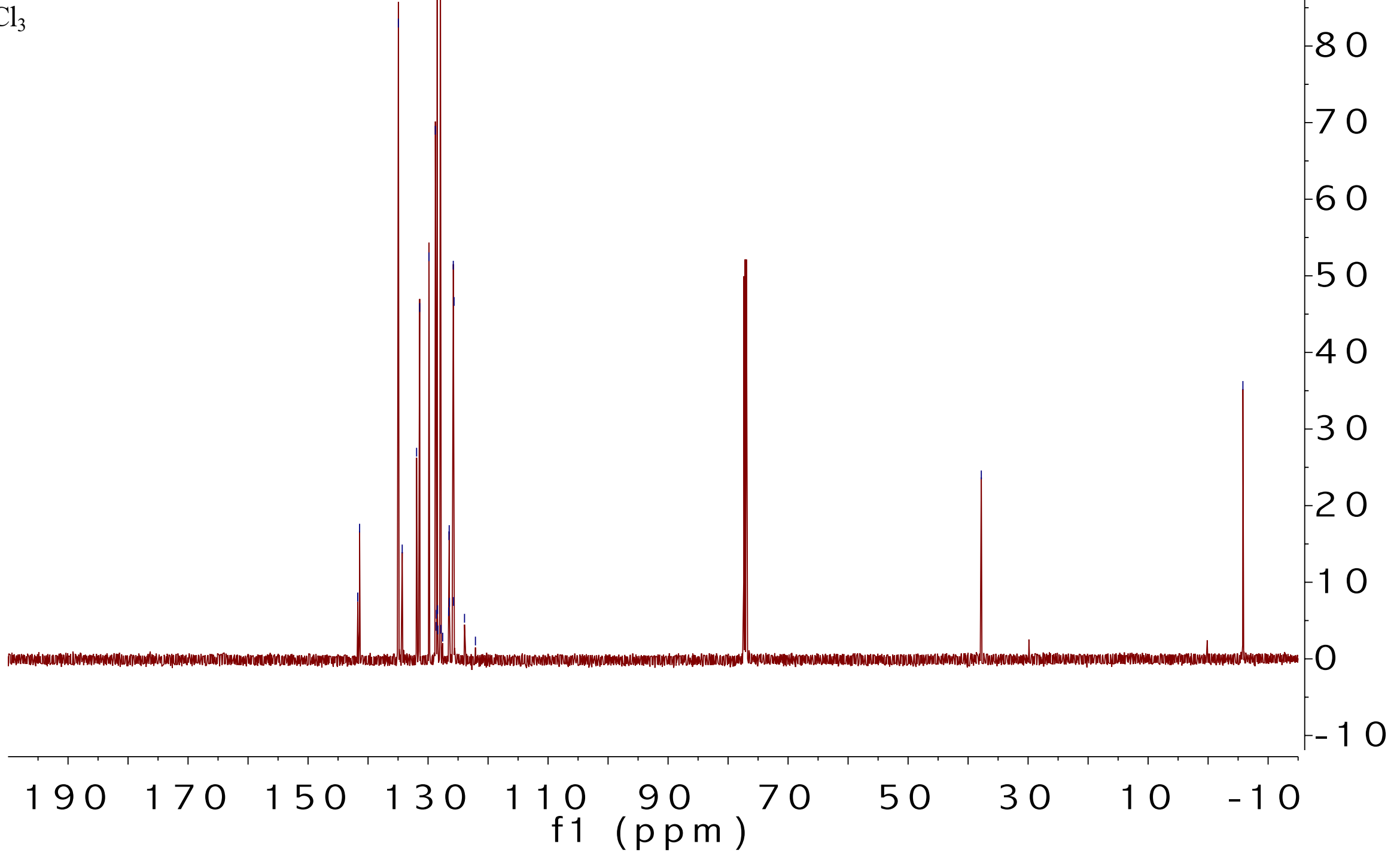

$\mathrm{S} 142$ 


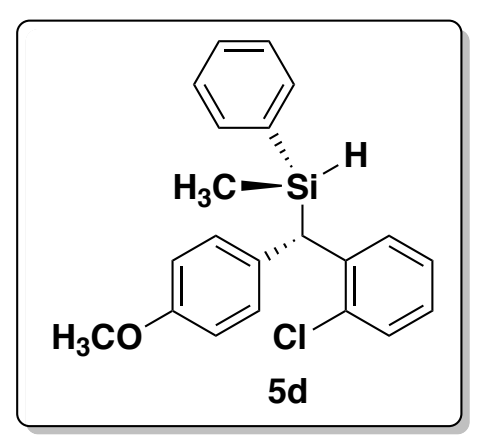




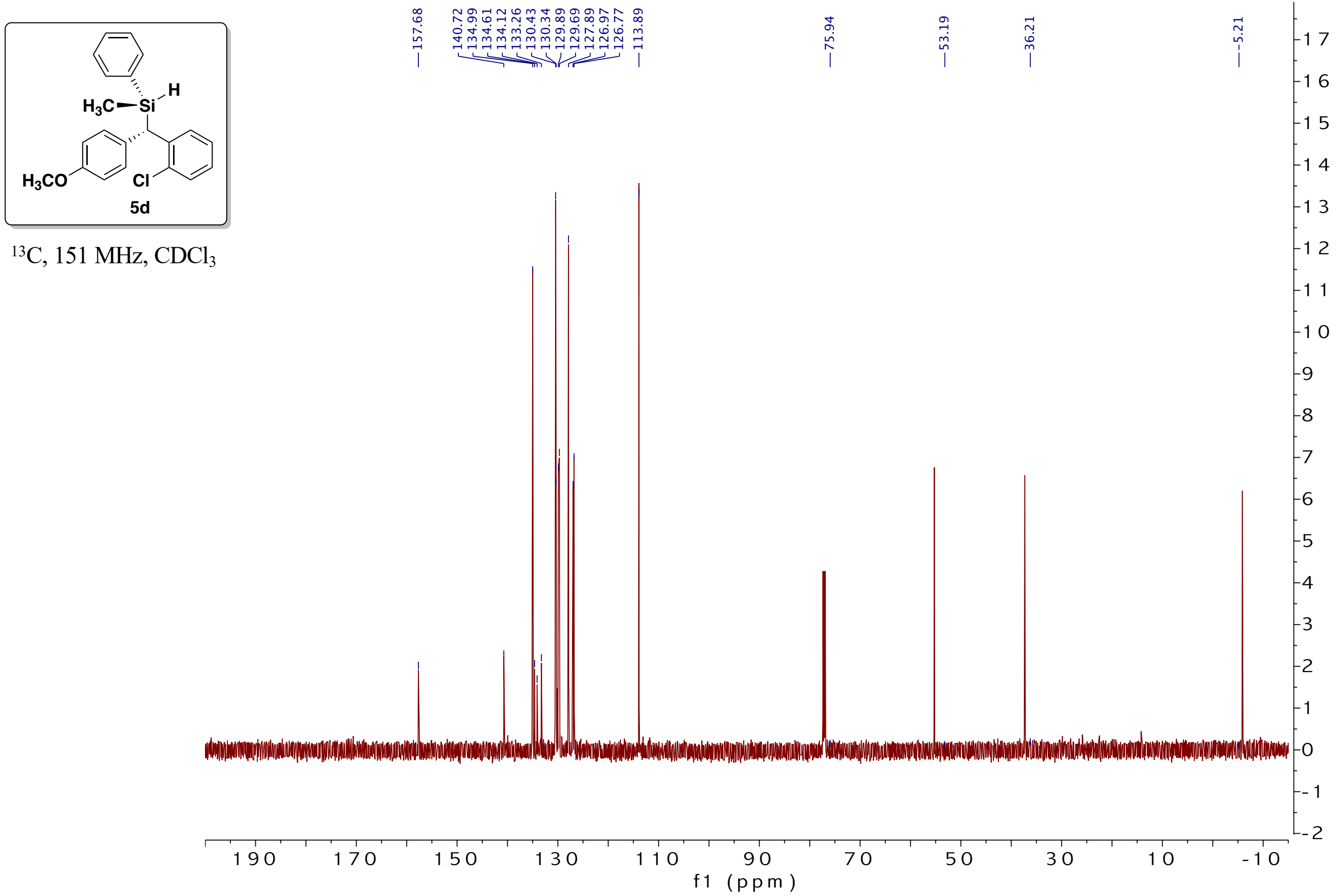

S144 

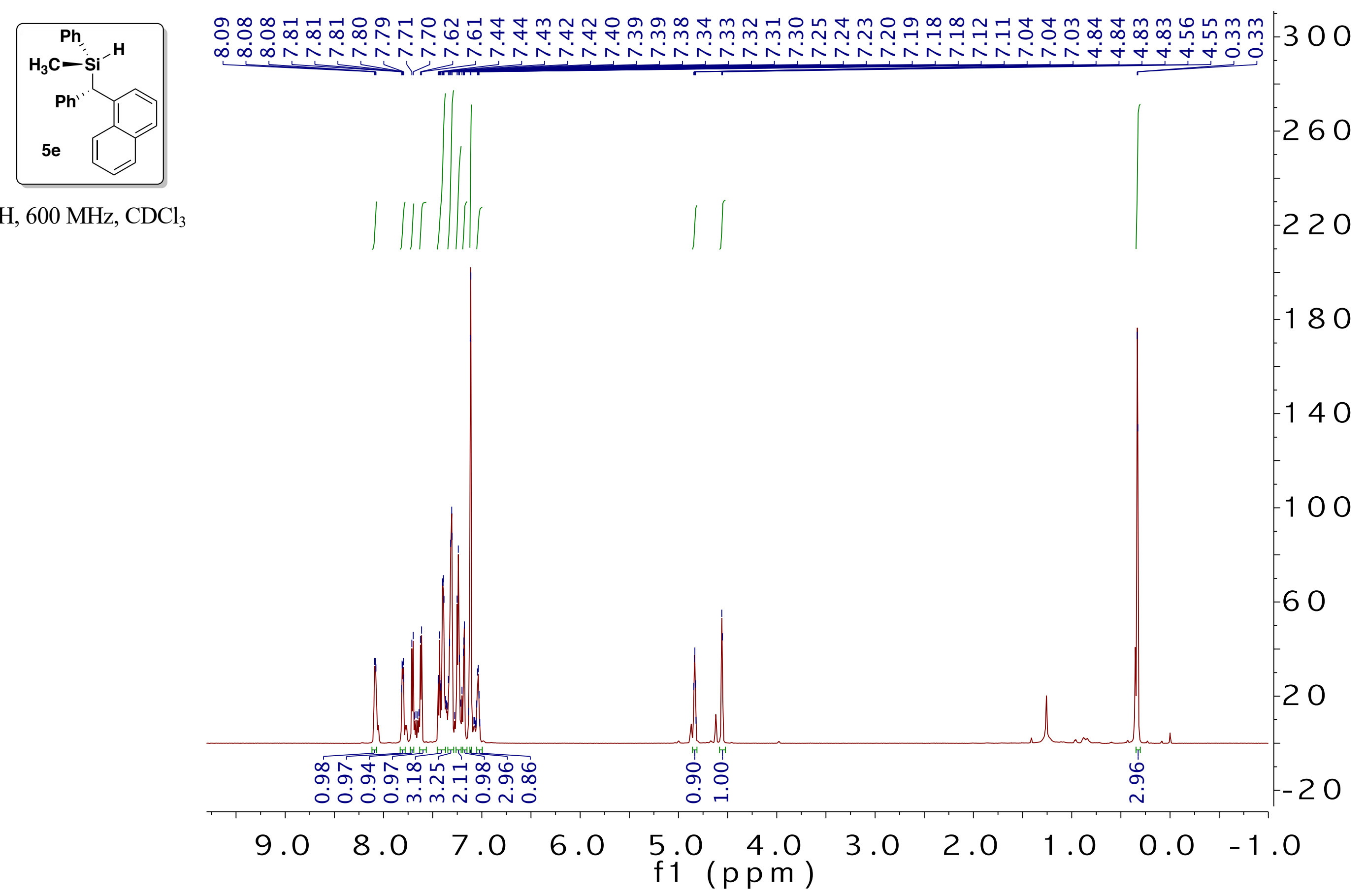

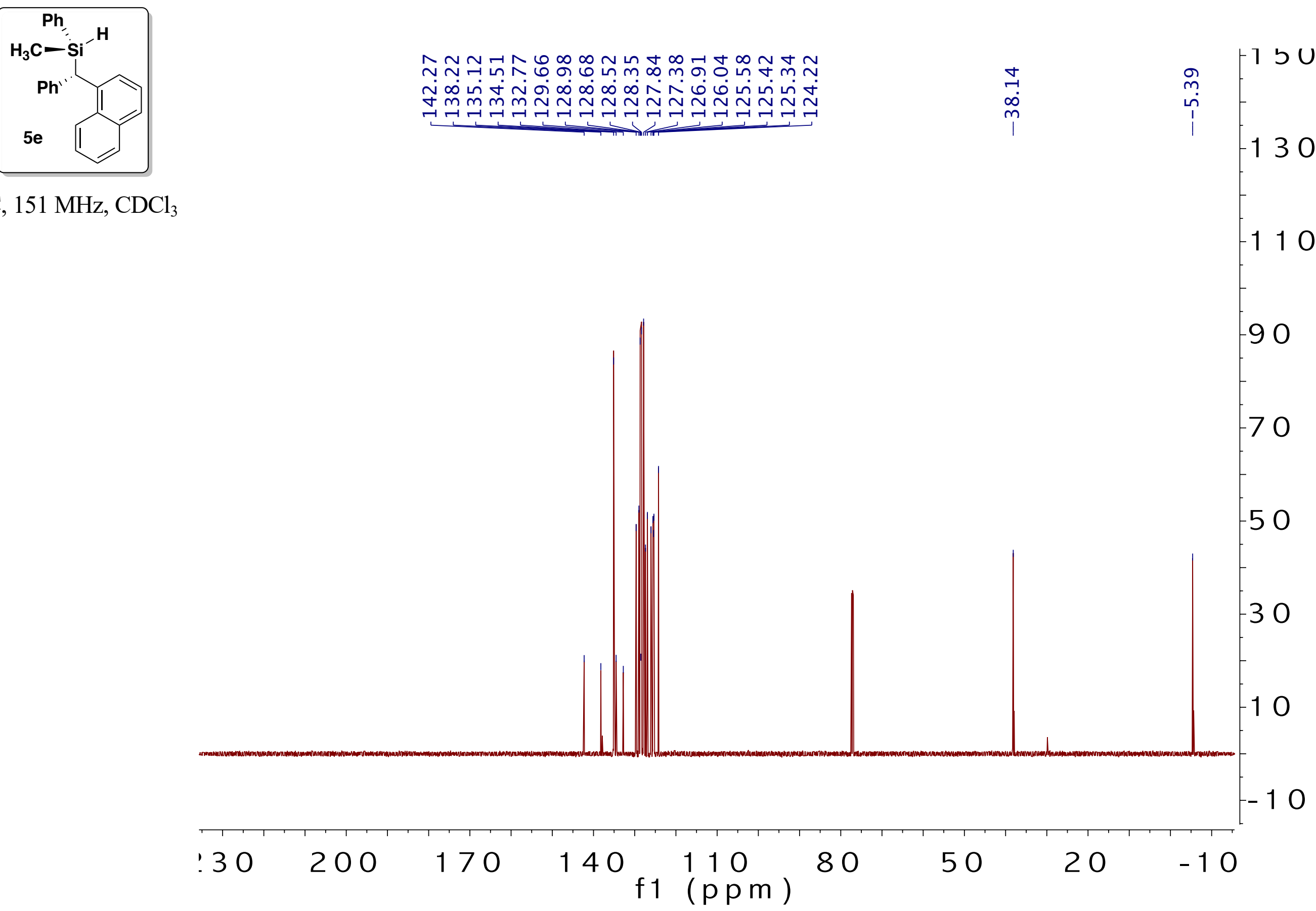

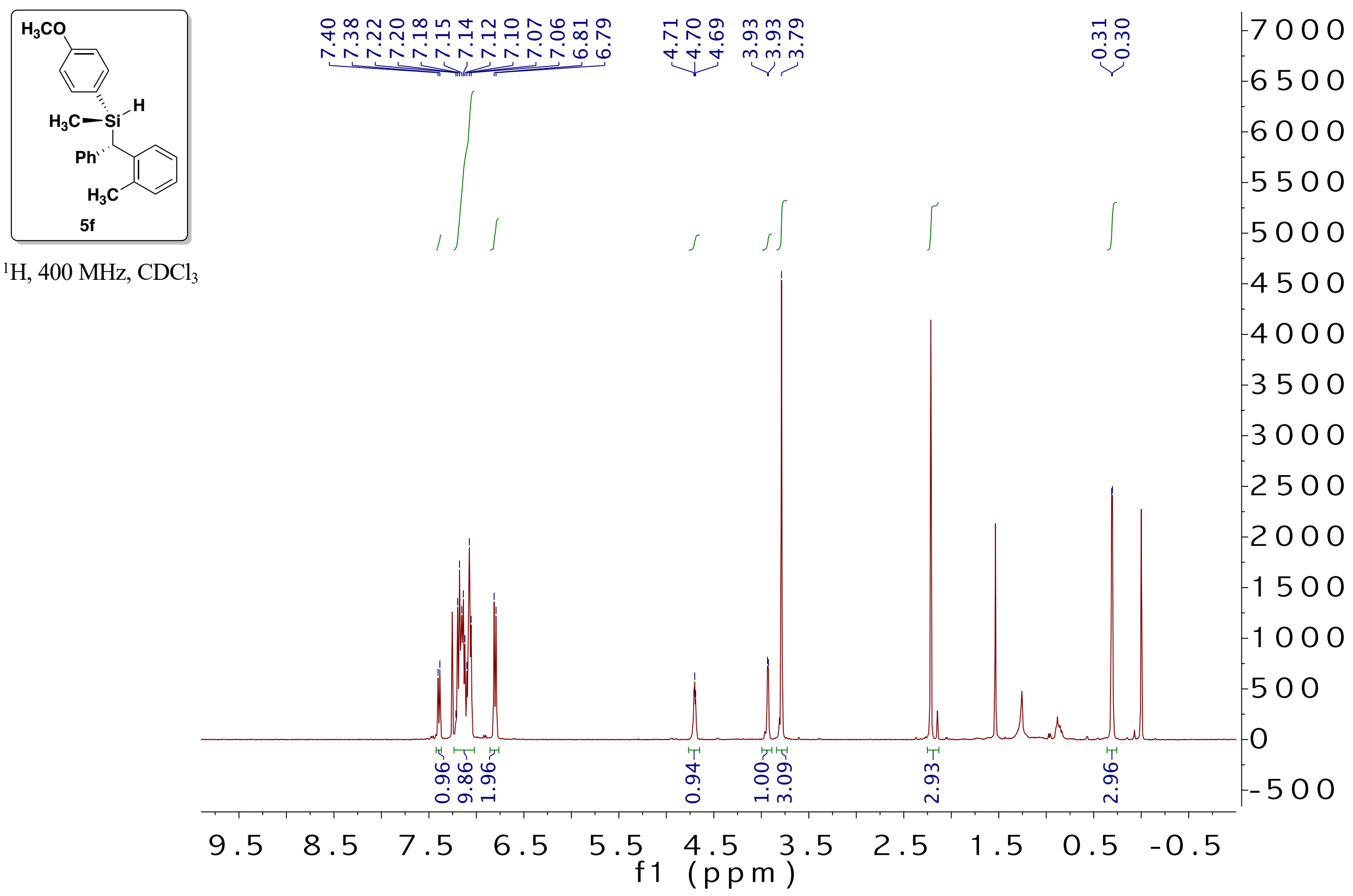


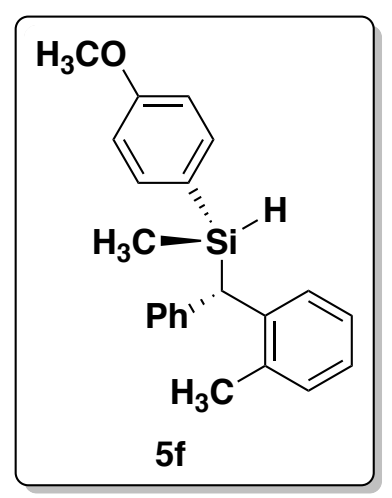

${ }^{13} \mathrm{C}, 151 \mathrm{MHz}, \mathrm{CDCl}_{3}$

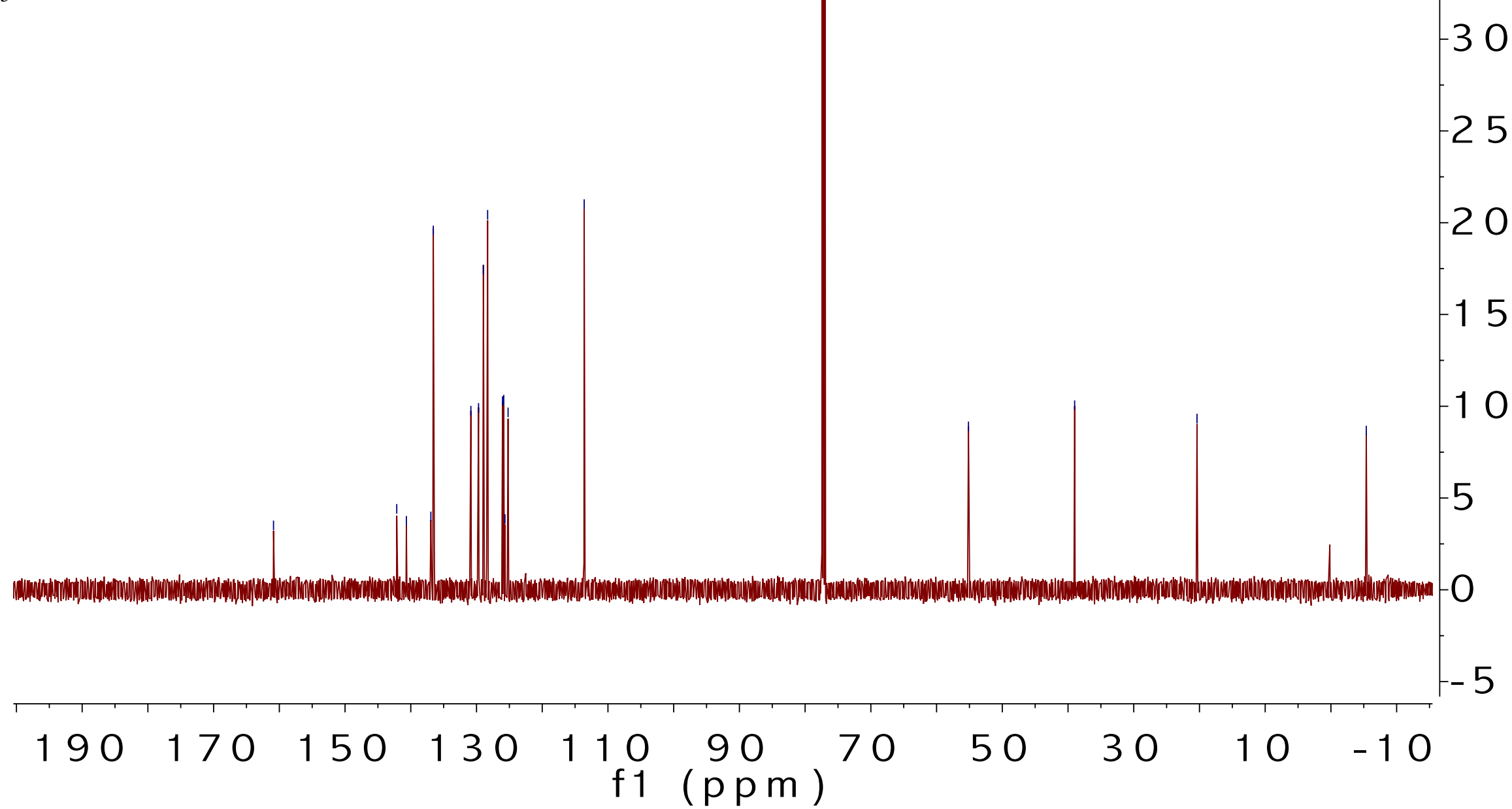




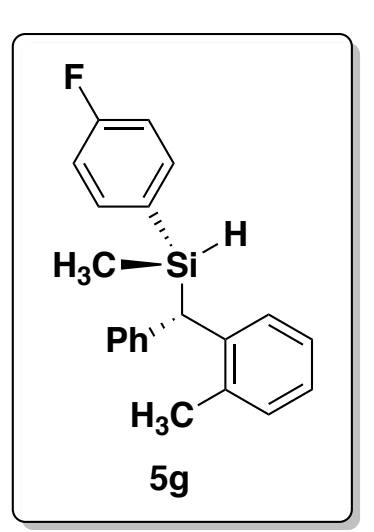

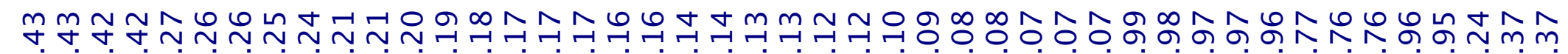

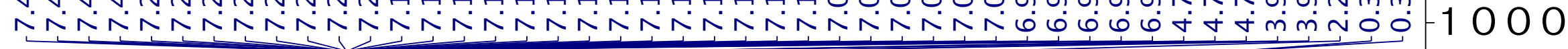

${ }^{1} \mathrm{H}, 600 \mathrm{MHz}, \mathrm{CDCl}_{3}$
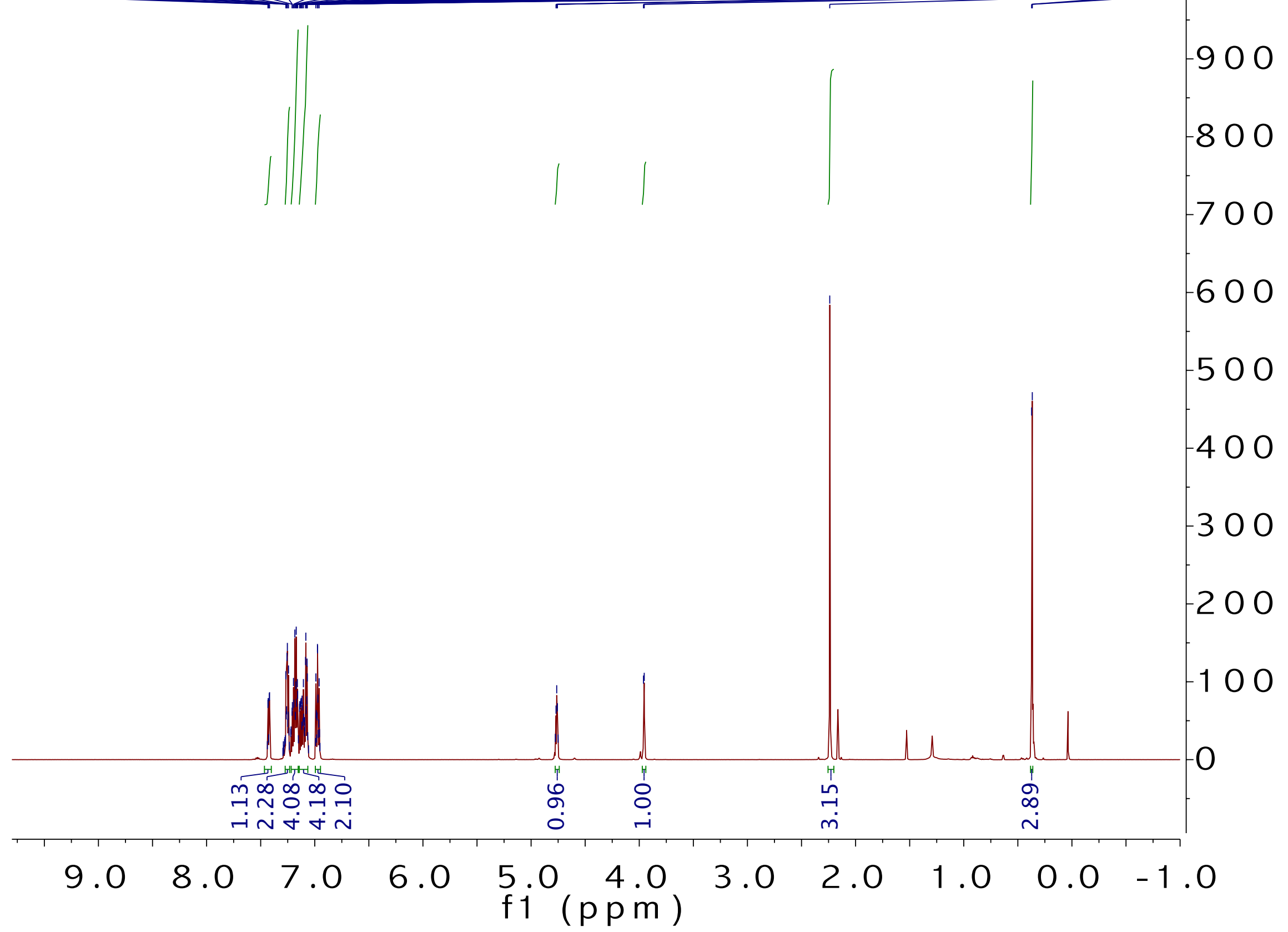


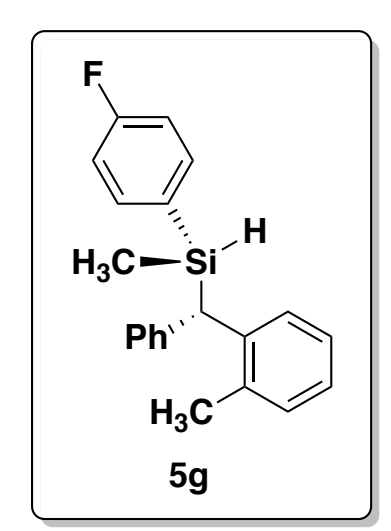

${ }^{13} \mathrm{C}, 151 \mathrm{MHz}, \mathrm{CDCl}_{3}$

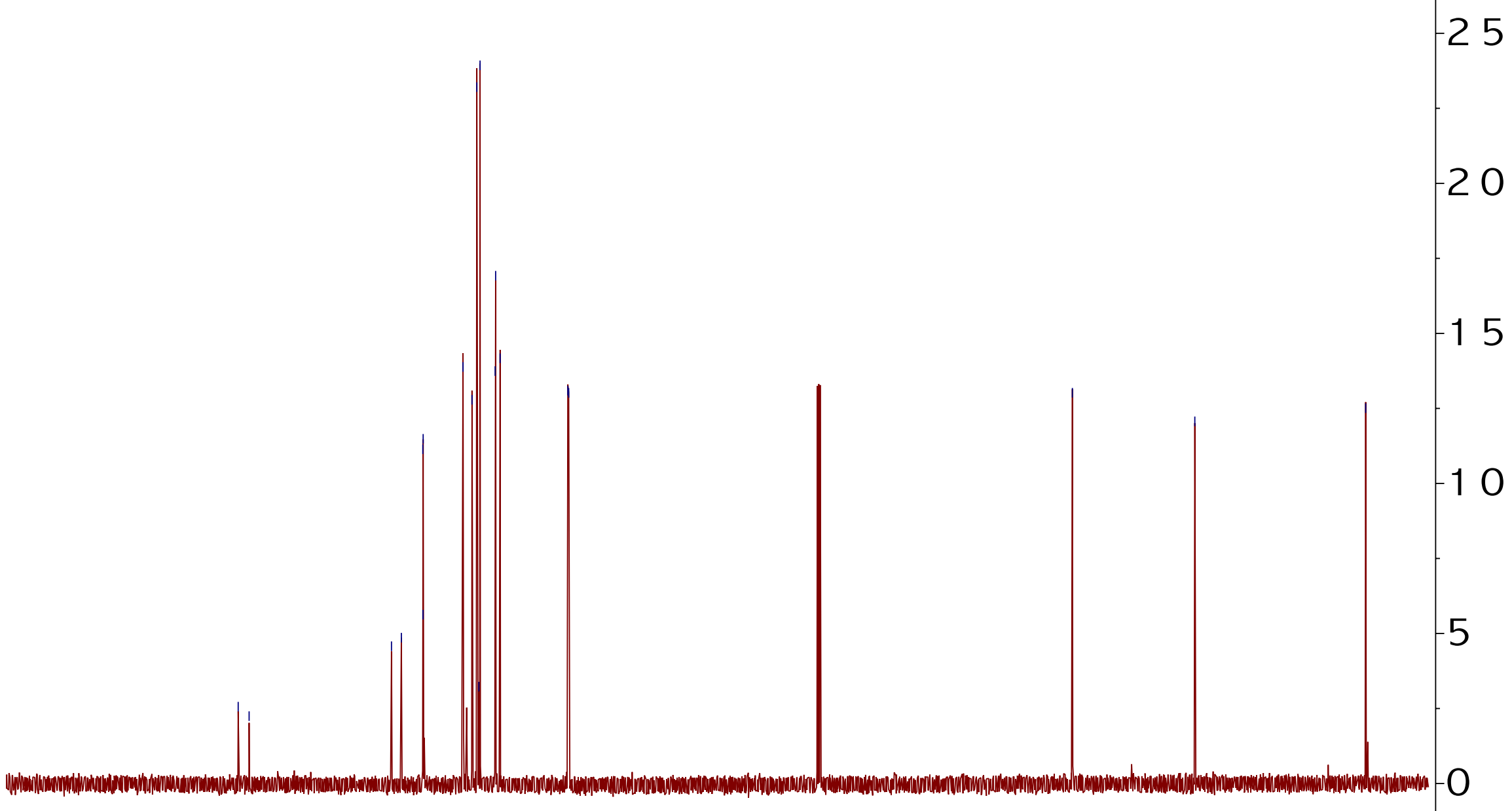

$$
\begin{array}{lllllllllll}
190 & 170 & 150 & 130 & 110 & 90 & 90 & 50 & 30 & 10 & -10
\end{array}
$$




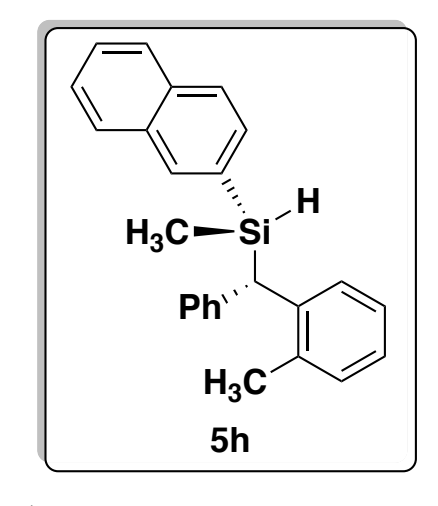

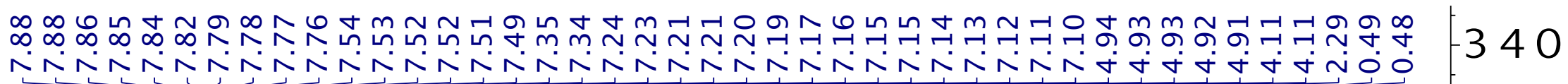

${ }^{1} \mathrm{H}, 600 \mathrm{MHz}, \mathrm{CDCl}_{3}$

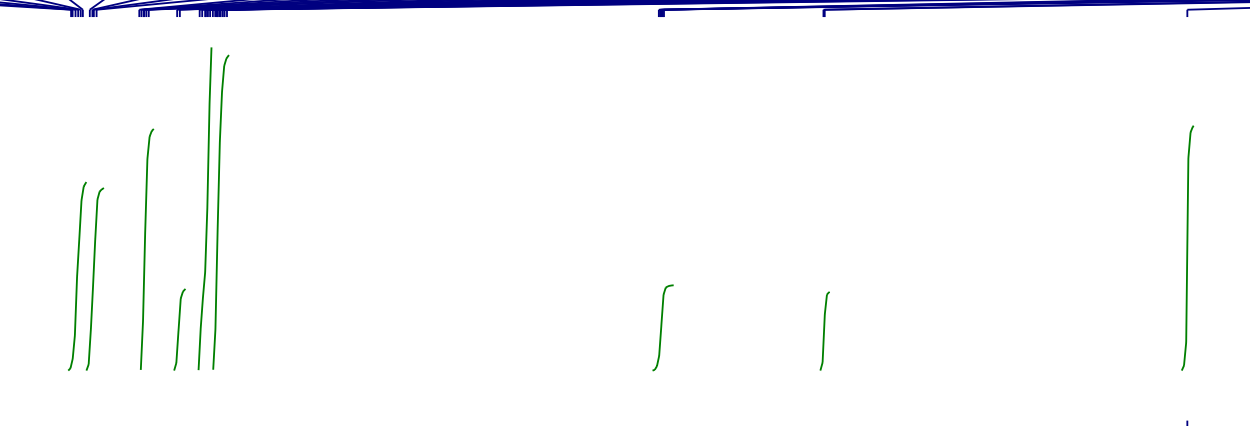

300

$-260$

220

180

140

100

$-60$

$-20$

$\begin{array}{lllllllllll}9.0 & 8.0 & 7.0 & 6.0 & 5.0 & 4.0 & 3.0 & 2.0 & 1.0 & 0.0 & -1.0\end{array}$ f1 (ppm) 


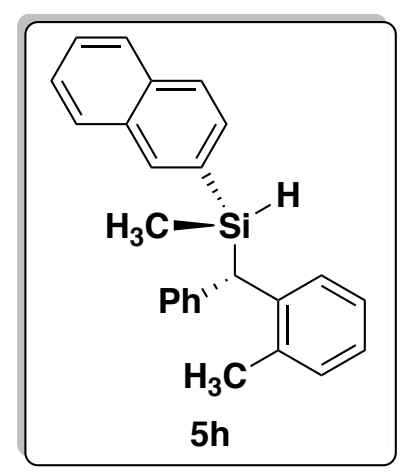




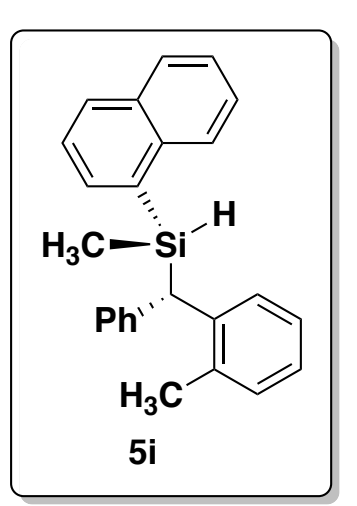

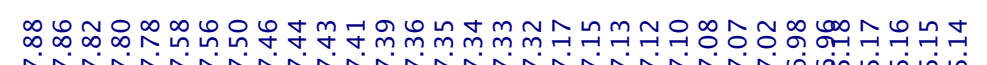

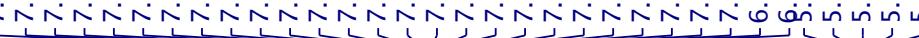

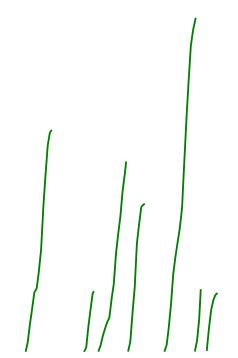

$-16000$

$-15000$

$-14000$

$-13000$

$-12000$

$-11000$

10000

$-9000$

$-8000$

$-7000$

$-6000$

$-5000$

$-4000$

$-3000$

$-2000$

$-1000$

$-1000$

9.0

$8.0 \quad 7.0 \quad 6.0$

$\begin{array}{cr}5.0 & 4.0 \\ f 1 & (p p m)\end{array}$

2.0

$\begin{array}{lll}1.0 & 0.0 & -1.0\end{array}$ 


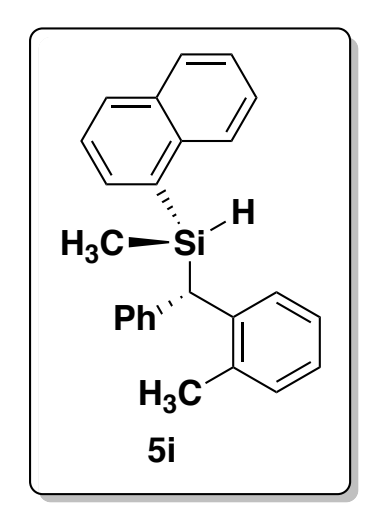

$5 \angle 4 \cup \cup u$

${ }^{13} \mathrm{C}, 100 \mathrm{MHz}, \mathrm{CDCl}_{3}$

$$
\begin{aligned}
& -23000 \\
& -22000 \\
& -21000 \\
& -20000 \\
& -19000 \\
& -18000 \\
& -17000 \\
& -16000 \\
& -15000 \\
& -14000 \\
& -13000 \\
& -12000 \\
& -11000 \\
& -10000 \\
& -9000 \\
& -8000 \\
& -7000 \\
& -6000 \\
& -5000 \\
& -4000 \\
& -3000 \\
& -2000 \\
& -1000
\end{aligned}
$$



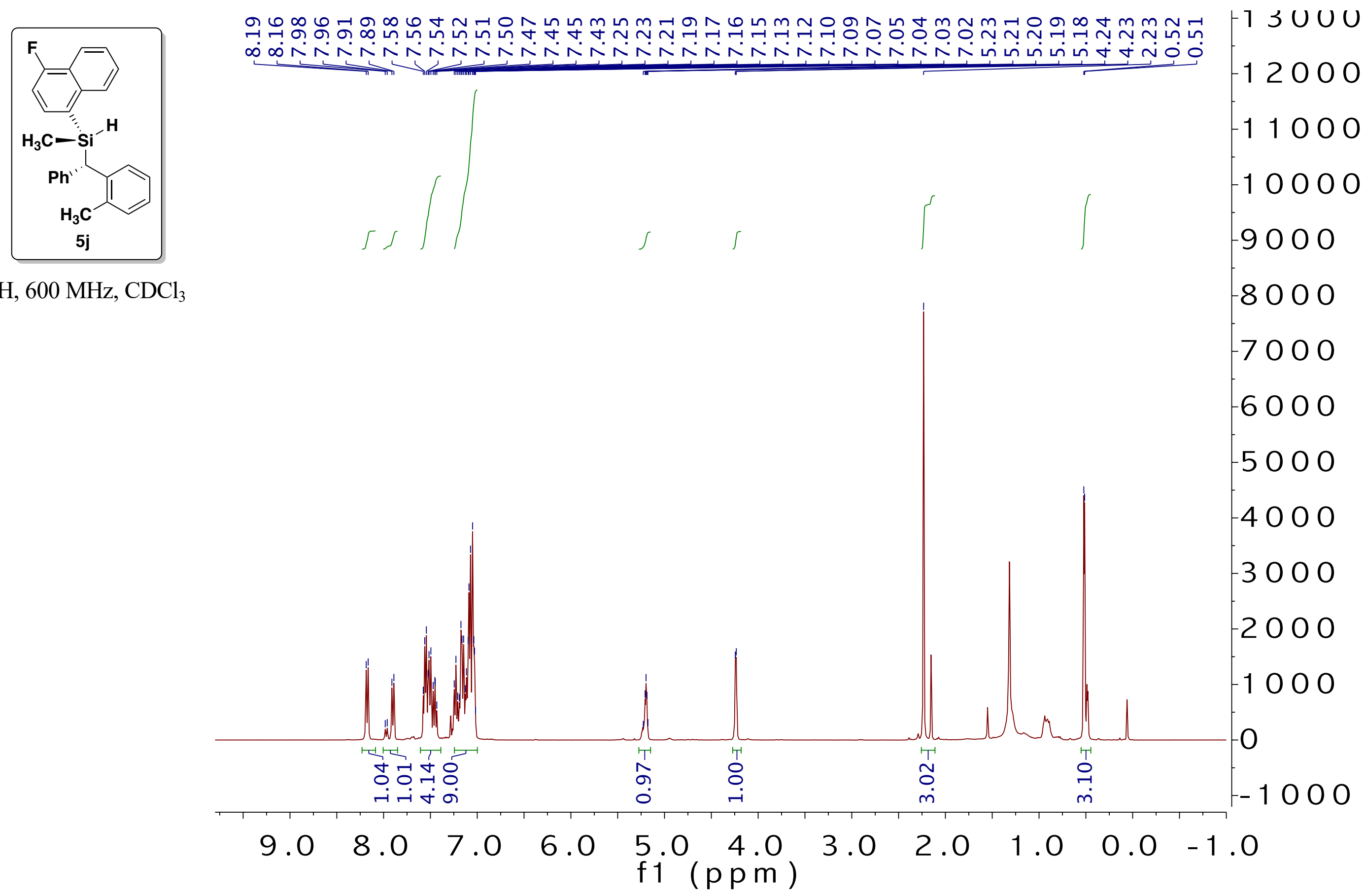

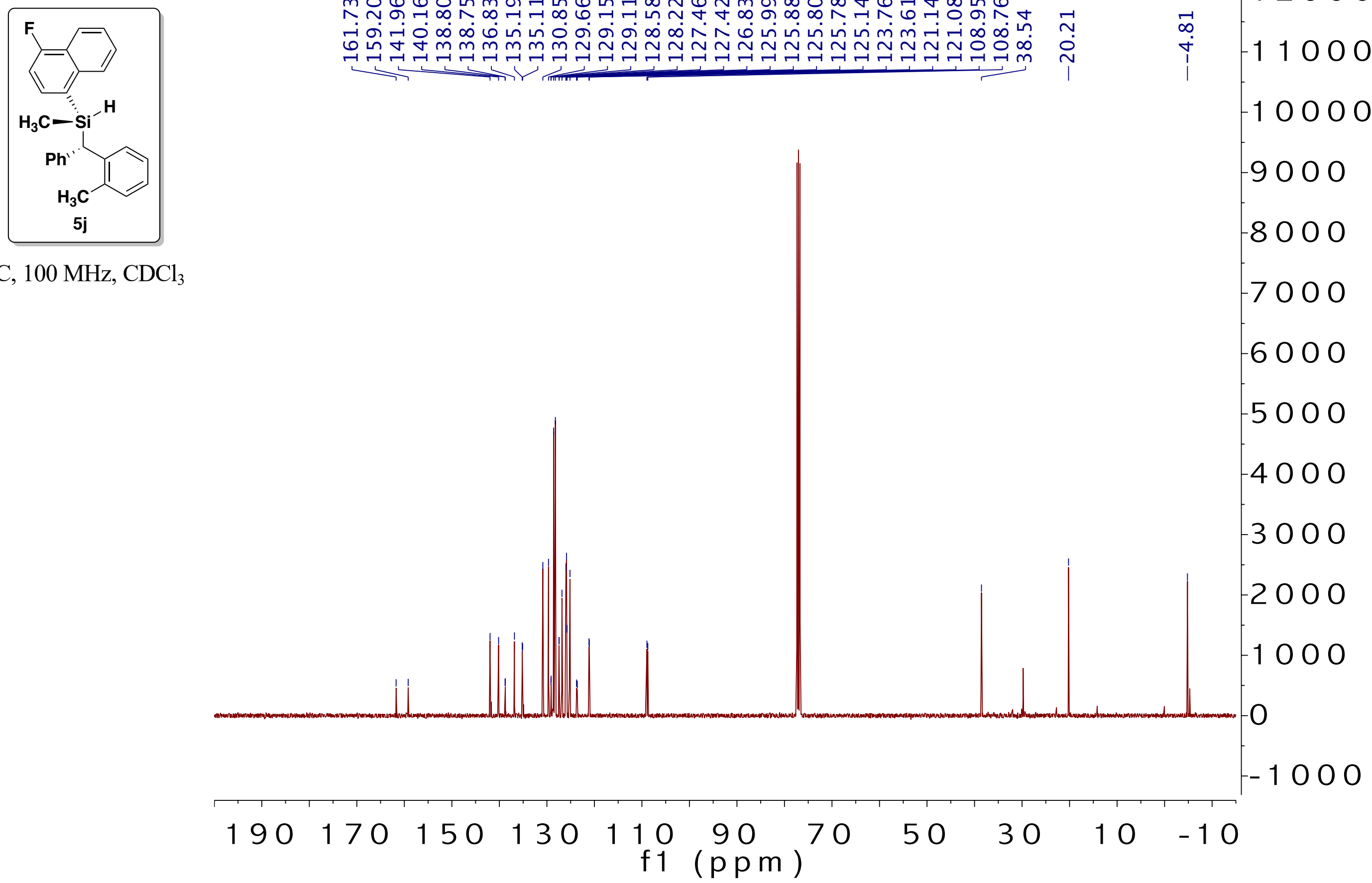


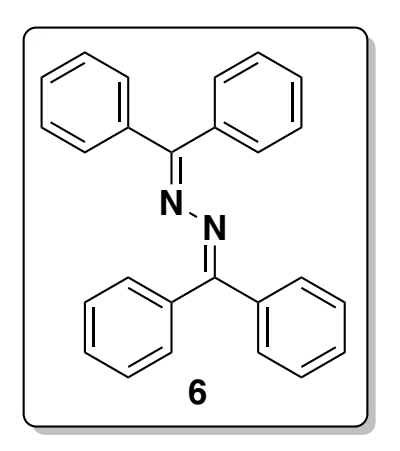

${ }^{1} \mathrm{H}, 600 \mathrm{MHz}, \mathrm{CDCl}_{3}$

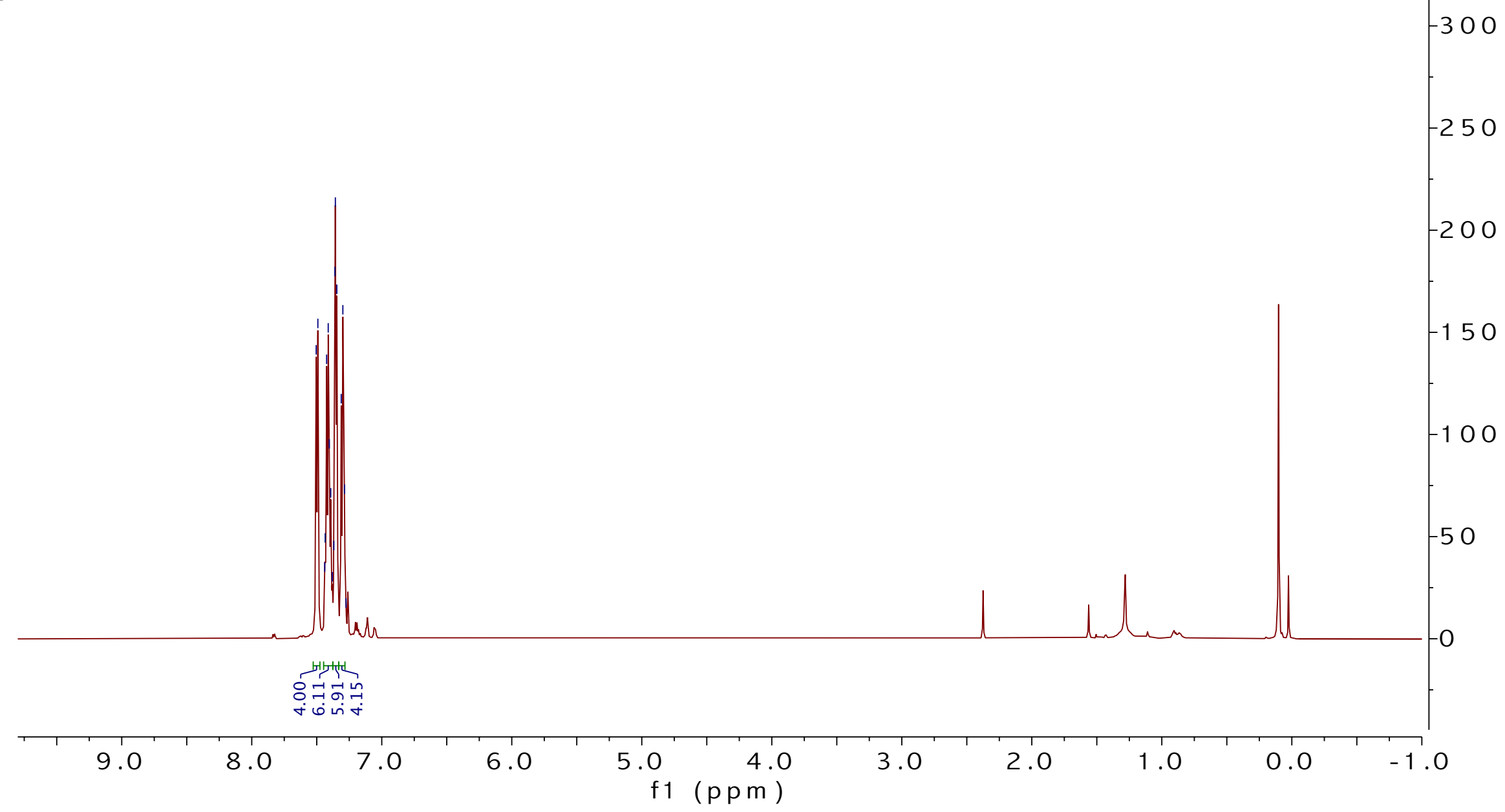



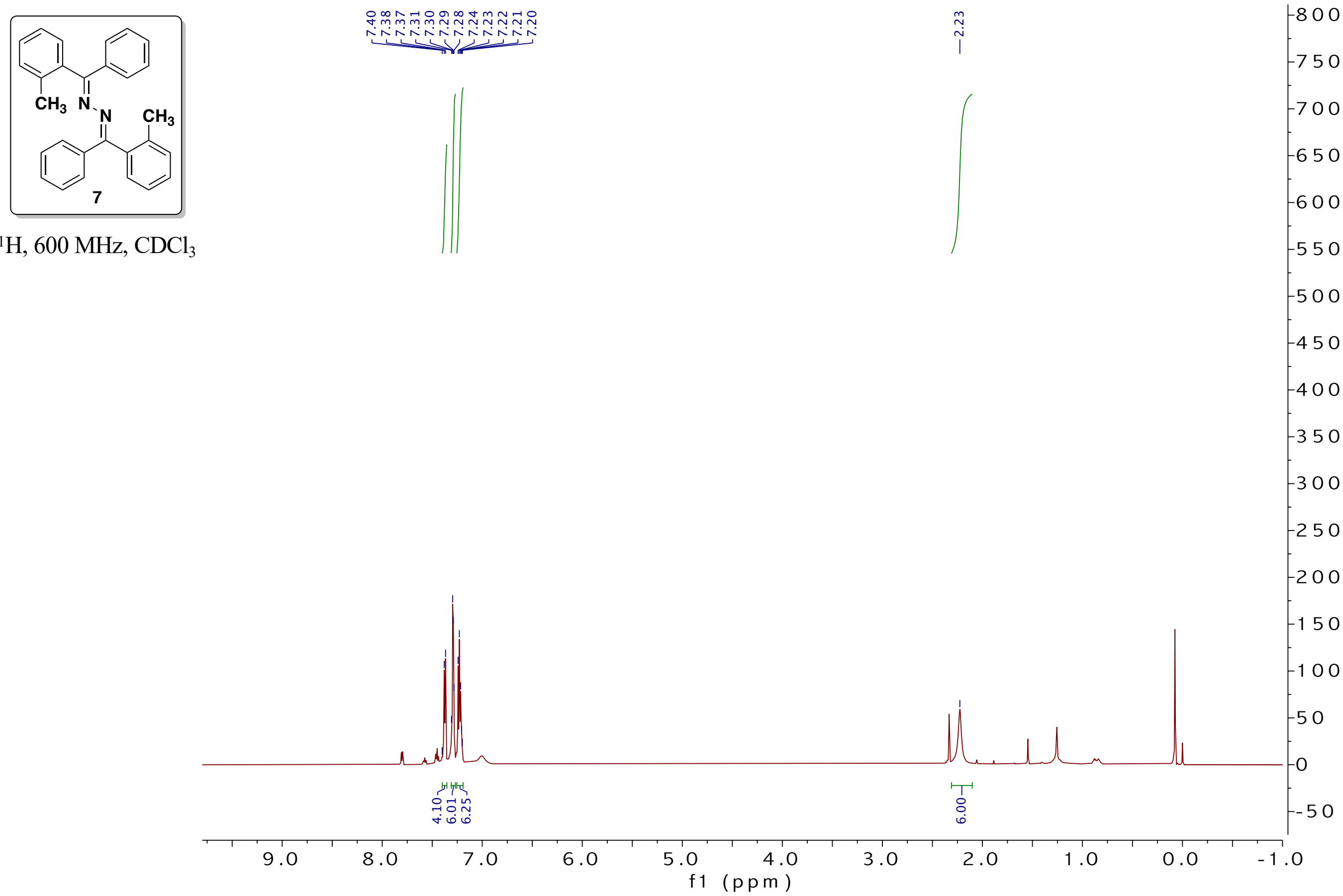


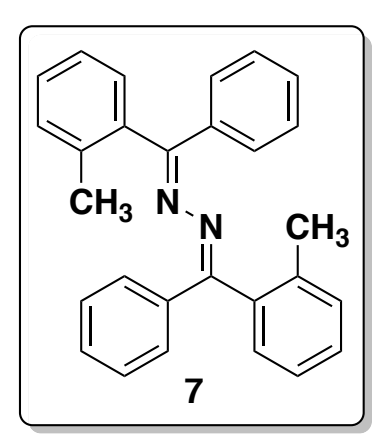

${ }^{13} \mathrm{C}, 151 \mathrm{MHz}, \mathrm{CDCl}_{3}$ $\mathrm{l}_{3}$ 

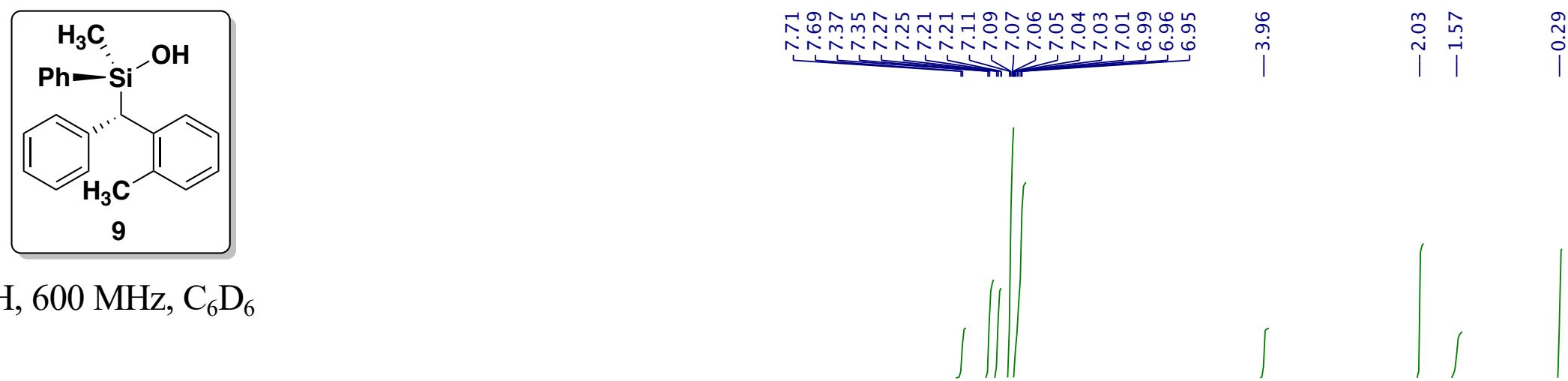

$-15000$

14000

13000

$-12000$

$-11000$

$-10000$

$-9000$

$-8000$

$-7000$

$-6000$

$-5000$

$-4000$

$-3000$

$-2000$

$-1000$ 


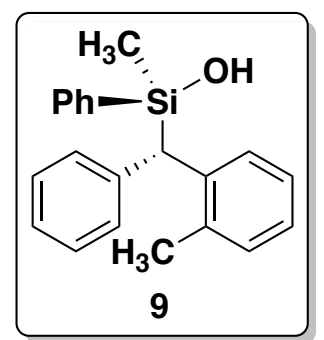

${ }^{13} \mathrm{C}, 151 \mathrm{MHz}, \mathrm{CDCl}_{3}$

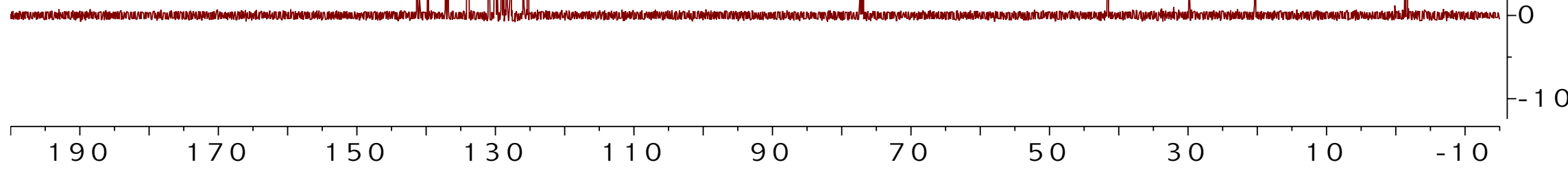




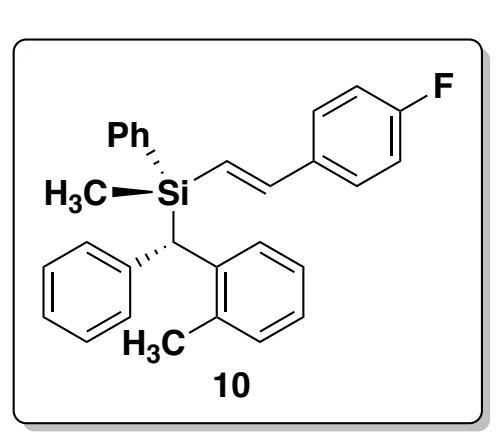

${ }^{1} \mathrm{H}, 600 \mathrm{MHz}, \mathrm{CDCl}_{3}$

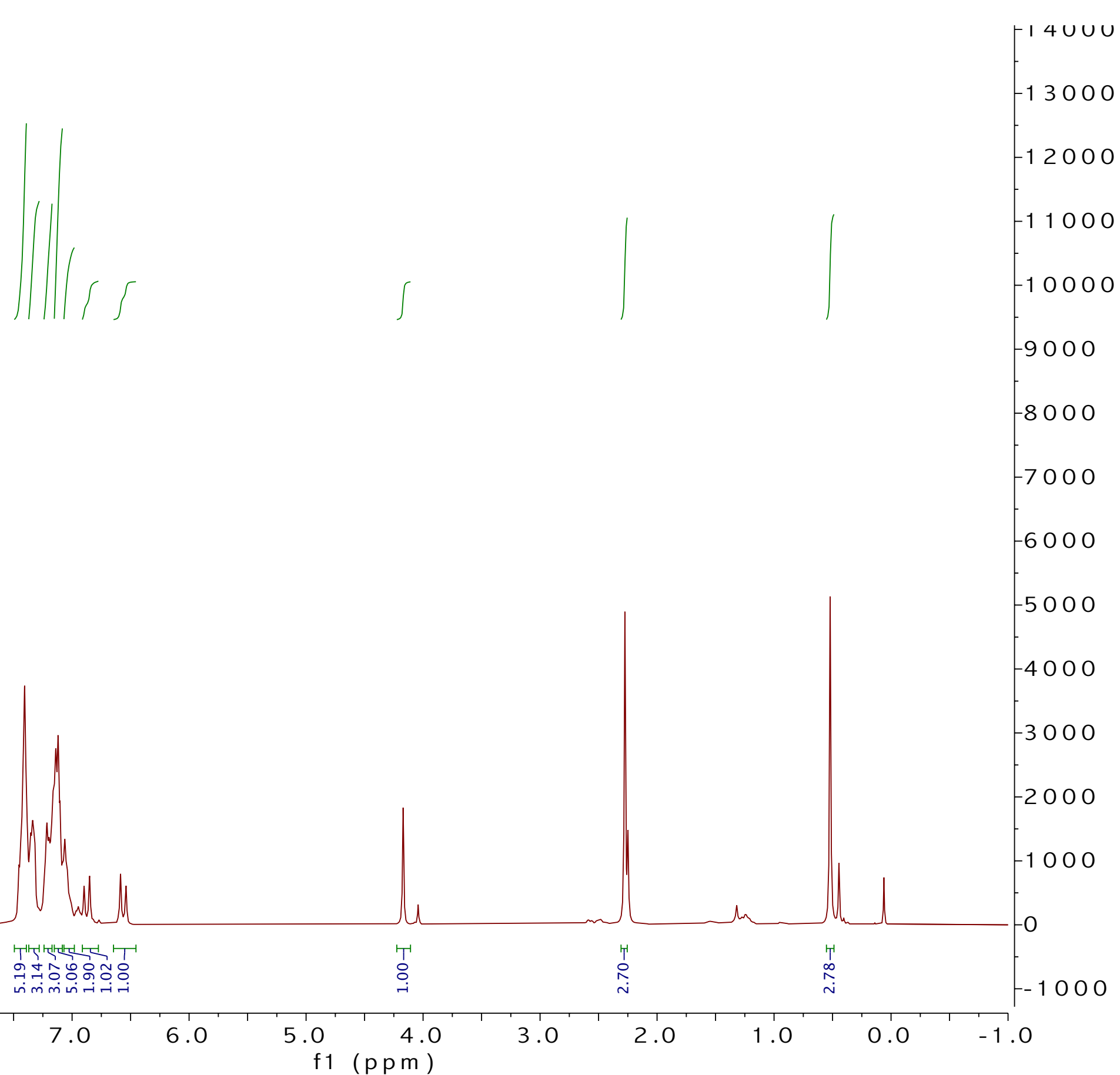




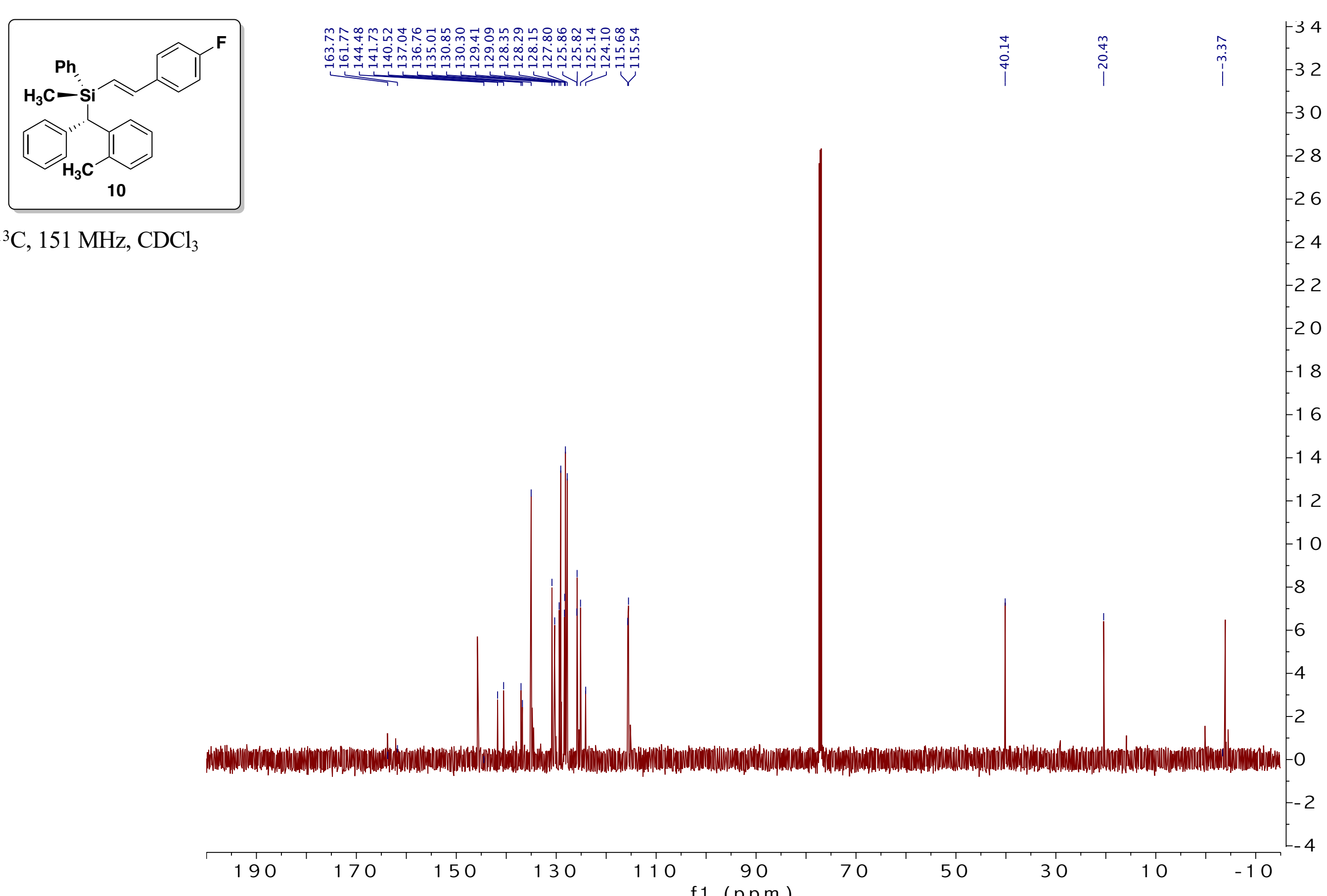

f1 (ppm) 

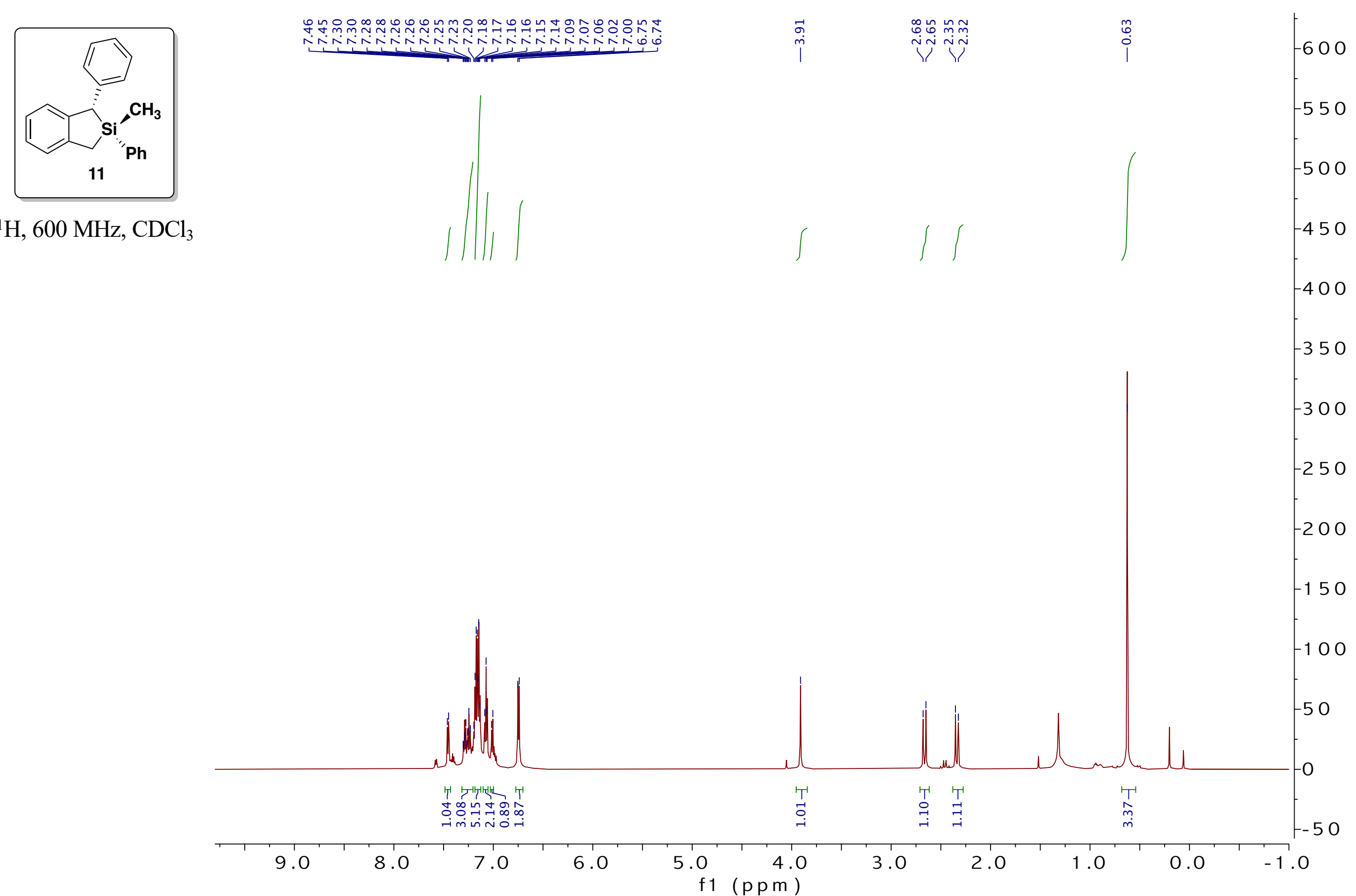


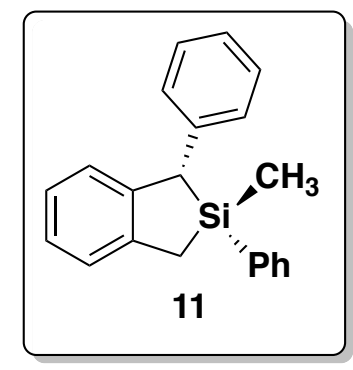

${ }^{13} \mathrm{C}, 151 \mathrm{MHz}, \mathrm{CDCl}_{3}$ 


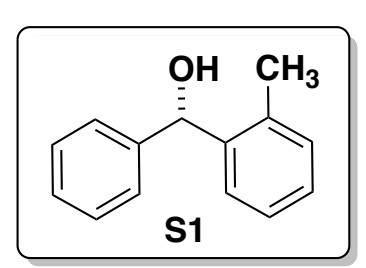

${ }^{1} \mathrm{H}, 600 \mathrm{MHz}, \mathrm{CDCl}_{3}$

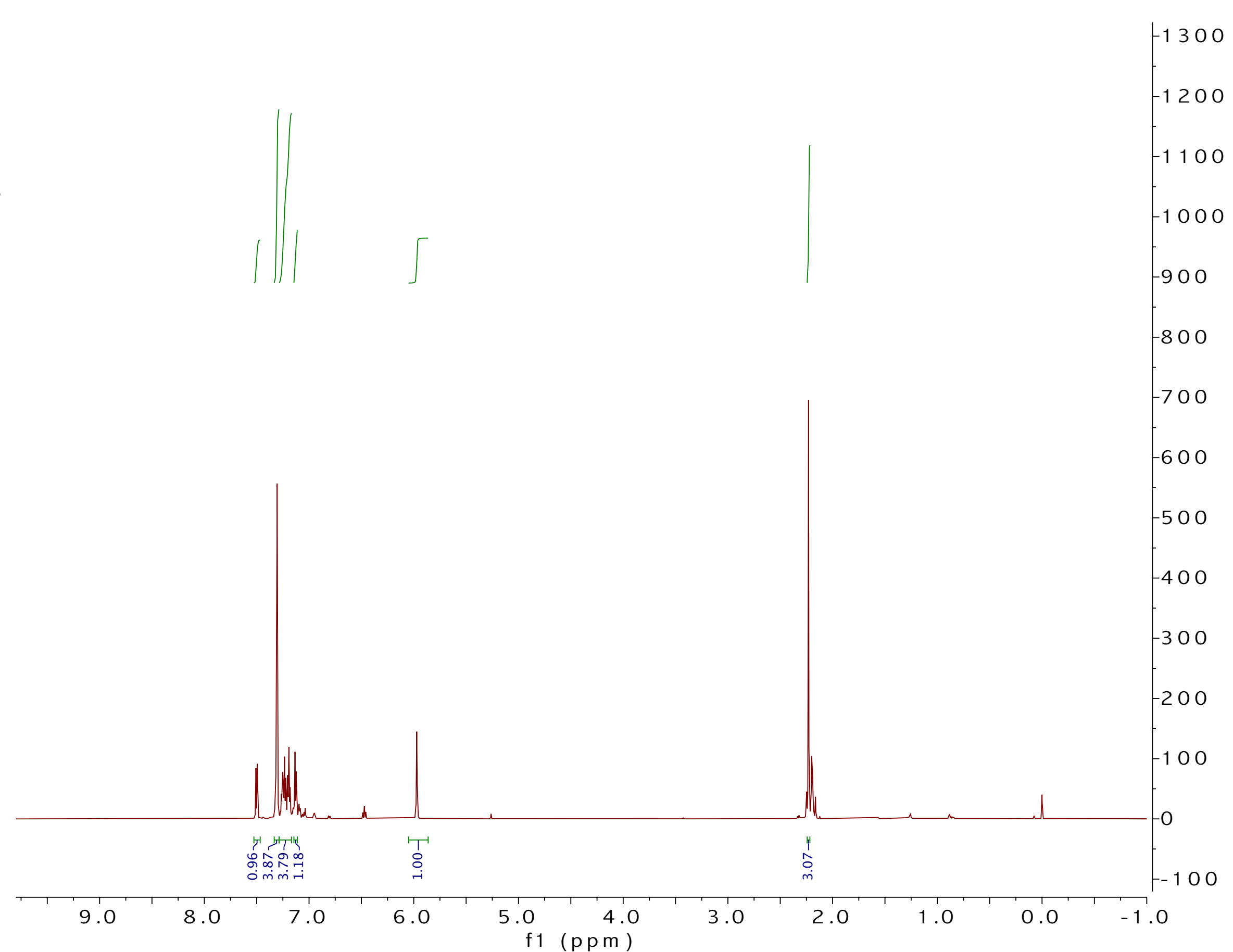

